\title{
Análise do Desempenho de Um Conjunto de Estimadores para os Coeficientes de Uma Equação Diferencial Parcial
}

Pedro Américo R. Campello de Freitas Penalber

\author{
DISSERTAÇÃO APRESENTADA \\ $\mathrm{AO}$ \\ Instituto De MatemáticA E EstatísticA \\ DA \\ Universidade DE SÃo PAUlo \\ PARA \\ OBTENÇÃO DO TÍTULO \\ DE \\ Mestre em CiÊnCIAS \\ Programa: Estatística \\ Orientador: Prof. Dr. José Carlos Simon de Miranda
}

São Paulo, fevereiro de 2016 


\section{Análise do Desempenho de Um Conjunto de Estimadores para os Coeficientes de Uma Equação Diferencial Parcial}

Esta versão da dissertação/tese contém as correções e alterações sugeridas pela Comissão Julgadora durante a defesa da versão original do trabalho, realizada em 03/06/2016. Uma cópia da versão original está disponível no Instituto de Matemática e Estatística da Universidade de São Paulo.

Comissão Julgadora:

- Prof. Dr. José Carlos Simon de Miranda (Presidente)/IME-USP

- Prof. Dr. Pedro Alberto Morettin/IME-USP

- Prof. Dr. Luis Koodi Hotta/UNICAMP 


\section{Resumo}

Penalber, P. A. R. C. F. Análise do Desempenho de Um Conjunto de Estimadores para os Coeficientes de Uma Equação Diferencial Parcial. 2016. 120 f. Dissertação (Mestrado) Instituto de Matemática e Estatística, Universidade de São Paulo, São Paulo, 2016.

Neste trabalho analisamos, de maneira descritiva utilizando simulações, o desempenho um conjunto de estimadores para os parâmetros funcionais $f, g, h$ e $k$ de uma EDP da forma $f \frac{\partial^{2} u}{\partial t^{2}}+g \frac{\partial u}{\partial t}+$ $h u=\frac{\partial}{\partial x}\left(k \frac{\partial u}{\partial x}\right)$. Para as simulações, selecionamos doze EDPs nas quais aplicaremos os estimadores as soluções dessas EDPs. Iremos medir o desempenho dos estimadores utilizando o erro quadrático entre as estimativas obtidas e os coeficientes conhecidos das EDPs. As estimativas dos coeficientes serão obtidas para soluções com e sem ruídos. Os estimadores propostos serão comparados com um grupo de estimadores diretos. Os programas para as simulações foram desenvolvidos com programas no repositório R, versão 3.2.3. Veremos que os estimadores propostos apresentaram um desempenho superior aos dos estimadores diretos, para soluções com e sem ruídos. Nas soluções com ruídos os estimadores propostos tiveram um desempenho mais significativo que para soluções sem ruídos.

Palavras-chave: Estimador, EDP. 


\section{Abstract}

Penalber, P. A. R. C. F. The Performance Analysis of a Set of Estimators for Coefficients from an Differential Equation Partial. 2016. 120 f. Dissertação (Mestrado) - Instituto de Matemática e Estatística, Universidade de São Paulo, São Paulo, 2016.

In this paper we shall analyze, on a descriptive way using simulations, the performance of a set of estimators for the coefficients $\mathrm{f}, \mathrm{g}, \mathrm{h}$ and $\mathrm{k}$ using the following. For the simulations, we select twelve EDPs in which we will apply the estimators from the solutions from the mentioned EDPs. We will measure the performance using the quadratic error between the estimates obtained and the known coefficients from the EDPs. The estimates will be obtained for solutions with and without noises. The proposed estimators will be compared with a group of direct estimators. The programs used for the simulations were developed with the programs in the repository $\mathrm{R}$, version 3.2.3. We shall see that the proposed estimators present a superior performance compared to the direct estimators, for solutions with or without noises. The proposed estimators have had a better performance in the solutions with noises than in the solutions without a noise.

Keywords: Estimator, PDE. 


\section{Sumário}

Lista de Abreviaturas vii

$\begin{array}{ll}\text { Lista de Figuras } & \text { ix }\end{array}$

Lista de Tabelas $\quad$ xi

1 Introdução $\quad 1$

1.1 Considerações preliminares $\ldots \ldots \ldots \ldots \ldots \ldots \ldots \ldots$

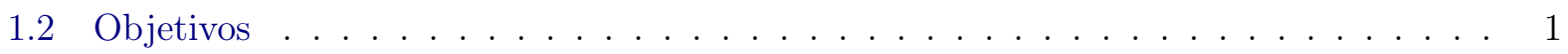

1.3 Organização do trabalho . . . . . . . . . . . . . . . . . . . 1

2 Equações Diferenciais Parciais $\quad 3$

2.1 Definição e classificação . . . . . . . . . . . . . . . . . . . . . 3

2.2 Condições iniciais e de contorno . . . . . . . . . . . . . . . . . 4

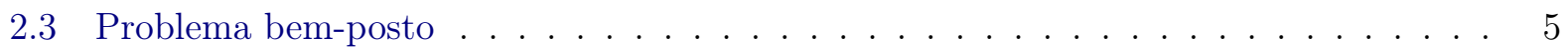

2.4 Equações de primeira ordem . . . . . . . . . . . . . . . . 6

2.5 Equações de segunda ordem . . . . . . . . . . . . . . . . . . 8

2.5 .1 Equação da onda . . . . . . . . . . . . . . . . . . . . . . . 9

2.5.2 Método da separação de variáveis . . . . . . . . . . . . . . . . . . 10

2.5.3 Equações elípticas . . . . . . . . . . . . . . . . . . . . . . . . . 13

3 Métodos Numéricos para Equações Diferenciais Parciais $\quad 15$

3.1 Método de diferenças finitas . . . . . . . . . . . . . . . . . . 15

3.2 Método das linhas . . . . . . . . . . . . . . . . . . . . . . 18

4 Ruídos $\quad 21$

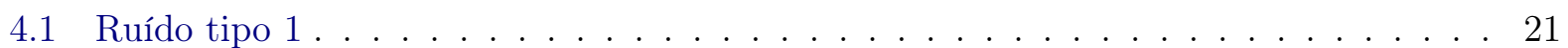

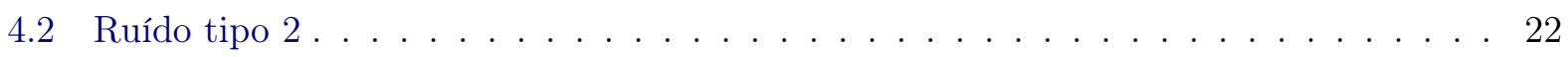

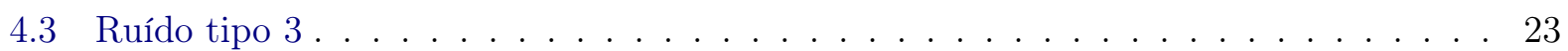

5 Estimadores $\quad 25$

5.1 Estimadores com integrais . . . . . . . . . . . . . . . . . 25

5.1 .1 Obtendo o estimador . . . . . . . . . . . . . . . . . . . 25

5.1 .2 Resultados principais . . . . . . . . . . . . . . . . . . . 28

5.1 .3 Observações . . . . . . . . . . . . . . . . . . . . . . . . . . . . 29

5.2 Estimadores diretos . . . . . . . . . . . . . . . . . . . . . . . . . . . . . 29 
6 Análise e comparação dos estimadores $\quad 31$

6.1 Equações selecionadas . . . . . . . . . . . . . . . . . . . . . 31

6.1 .1 Soluções analíticas . . . . . . . . . . . . . . . . . . . . . . 31

6.1 .2 Soluções numéricas . . . . . . . . . . . . . . . . . . . . . . . . 39

6.2 Procedimento de simulação . . . . . . . . . . . . . . . . . . . . . 41

6.2 .1 Procedimento de simulação . . . . . . . . . . . . . . . . . . . . . 42

6.3 Análise dos resultados . . . . . . . . . . . . . . . . . . . . . 44

6.3 .1 Seleção das soluções . . . . . . . . . . . . . . . . . . . . . . . 44

6.3.2 Comparação entre os estimadores . . . . . . . . . . . . . . . . . . . . 49

6.3 .3 Comparação entre os ruídos . . . . . . . . . . . . . . . . . . . 50

7 Considerações Finais $\quad 101$

7.1 Sugestões para Pesquisas Futuras . . . . . . . . . . . . . . . . . . . . . . 101

$\begin{array}{ll}\text { Referências Bibliográficas } & 103\end{array}$ 


\title{
Lista de Abreviaturas
}

\author{
EDP Equação diferencial parcial \\ EDO Equação diferencial ordinária \\ EIDP Equação integro-diferencial parcial
}




\section{Lista de Figuras}

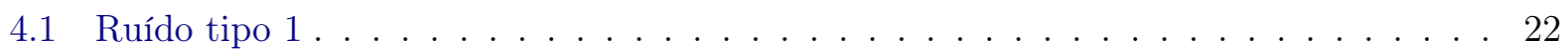

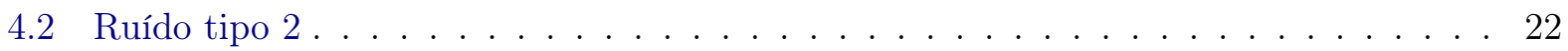

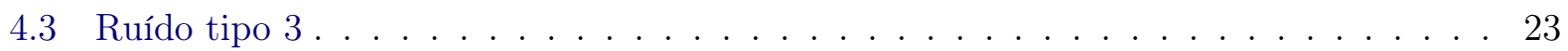

6.1 Determinante utilizado nos estimadores da equação (6.4) para as soluções 1,2 , 4 e $5 \quad 45$

6.2 Estimativa funções $F$ e $G$ da equação (6.4) com estimadores diretos para as soluções

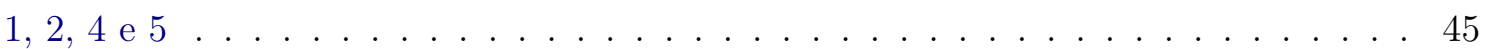

6.3 Estimativa funções $H$ e $K$ da equação (6.4) com estimadores diretos para as soluções

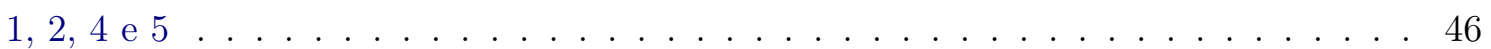

6.4 Determinante utilizado nos estimadores da equação (6.4) para as soluções $1,3,5,6$. 46

6.5 Estimativa funções $F$ e $G$ da equação (6.4) com estimadores diretos para as soluções

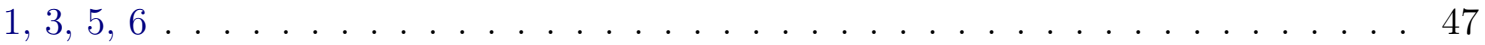

6.6 Estimativa funções $H$ e $K$ da equação (6.4) com estimadores diretos para as soluções

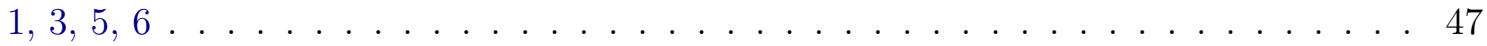

6.7 Determinante utilizado nos estimadores da equação $(6.12 \mathrm{a}) \quad \ldots . \ldots$. . . . . . . . 48

6.8 Estimativa funções $F$ e $G$ da equação $(6.12 \mathrm{a}) \ldots \ldots \ldots$

6.9 Estimativa funções $H$ e $K$ da equação $(6.12 \mathrm{a}) \ldots \ldots \ldots$. . . . . . . . . . . . . . 49

6.10 Comparação dos erros quadráticos para soluções com ruído sem ruído . . . . . . . . . 65

6.11 Comparação dos erros quadráticos para soluções com ruído sem ruído, usando $t=$

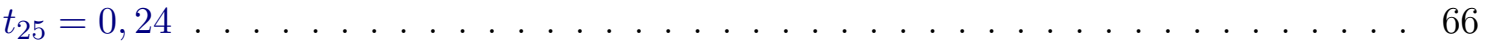

6.12 Comparação dos erros quadráticos para soluções com ruído sem ruído, usando $t=$ $t_{75}=0,74 \ldots \ldots \ldots \ldots \ldots \ldots \ldots \ldots \ldots \ldots \ldots \ldots \ldots \ldots \ldots \ldots \ldots \ldots \ldots \ldots$

6.13 Comparação dos erros quadráticos para soluções com ruído 1,1 ruído . . . . . . . . . 68

6.14 Comparação dos erros quadráticos para soluções com ruído 1, 1 ruído, usando $t=$

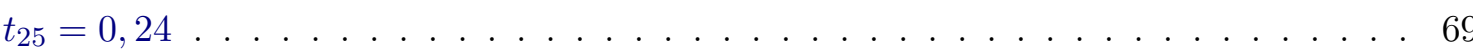

6.15 Comparação dos erros quadráticos para soluções com ruído 1, 1 ruído, usando $t=$

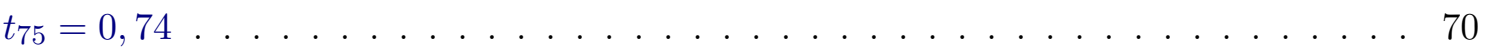

6.16 Comparação dos erros quadráticos para soluções com ruído 1, 100 ruídos . . . . . . 71

6.17 Comparação dos erros quadráticos para soluções com ruído 1, 1000 ruídos . . . . . 72

6.18 Comparação dos erros quadráticos para soluções com ruído 2,1 ruído . . . . . . . . . 73

6.19 Comparação dos erros quadráticos para soluções com ruído 2, 1 ruído, usando $t=$

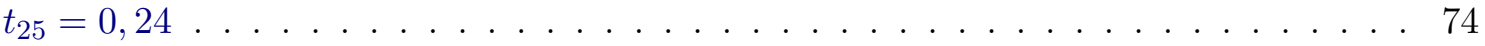

6.20 Comparação dos erros quadráticos para soluções com ruído 2,1 ruído, usando $t=$

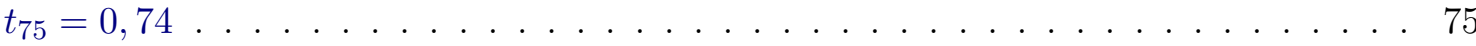


6.21 Comparação dos erros quadráticos para soluções com ruído 2, 100 ruídos . . . . . 76

6.22 Comparação dos erros quadráticos para soluções com ruído 2, 1000 ruídos . . . . . . 77

6.23 Comparação dos erros quadráticos para soluções com ruído 3,1 ruído . . . . . . . . . 78

6.24 Comparação dos erros quadráticos para soluções com ruído 3,1 ruído, usando $t=$

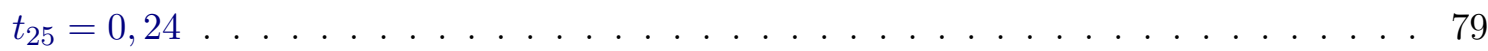

6.25 Comparação dos erros quadráticos para soluções com ruído 3,1 ruído, usando $t=$

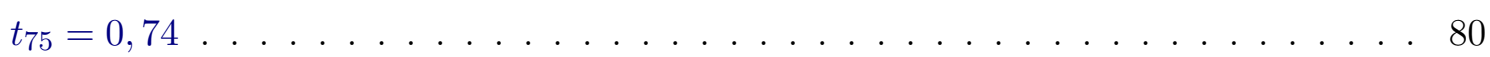

6.26 Comparação dos erros quadráticos para soluções com ruído 3, 100 ruídos . . . . . . . 81

6.27 Comparação dos erros quadráticos para soluções com ruído 3, 1000 ruídos . . . . . . 82

6.28 Comparação dos erros quadráticos para soluções com ruído 1 e 3,1 ruído . . . . . . . 83

6.29 Comparação dos erros quadráticos para soluções com ruído 1 e 2, 1 ruído . . . . . . . 84

6.30 Comparação dos erros quadráticos para soluções com ruído 2 e 3,1 ruído . . . . . . . 85

6.31 Erros quadráticos equações 1 e 2 , ruído $1 \ldots \ldots$. . . . . . . . . . 86

6.32 Erros quadráticos equações 3 e 4 , ruído $1 \ldots \ldots$. . . . . . . . . . 86

6.33 Erros quadráticos equações 5 e 6 , ruído $1 \ldots \ldots$. . . . . . . . . . . . 87

6.34 Erros quadráticos equações 7 e 8 , ruído $1 \ldots \ldots \ldots$. . . . . . . . 87

6.35 Erros quadráticos equações 9 e 10a, ruído $1 \ldots \ldots$. . . . . . . . . 88

6.36 Erros quadráticos equações $10 \mathrm{~b}$ e 11a, ruído $1 \ldots \ldots$. . . . . . . . . . 88

6.37 Erros quadráticos equações 11 b e 11 c, ruído $1 \ldots \ldots$. . . . . . . . . . . 89

6.38 Erros quadráticos equações 12 a e 12 b, ruído $1 \ldots$. . . . . . . . . . . . . . 89

6.39 Erros quadráticos equações 12 c, ruído $1 \ldots \ldots$. . . . . . . . . . . . 90

6.40 Erros quadráticos equações 1 e 2 , ruído $2 \ldots \ldots$. . . . . . . . . . . 90

6.41 Erros quadráticos equações 3 e 4 , ruído $2 \ldots \ldots$. . . . . . . . . . . . 91

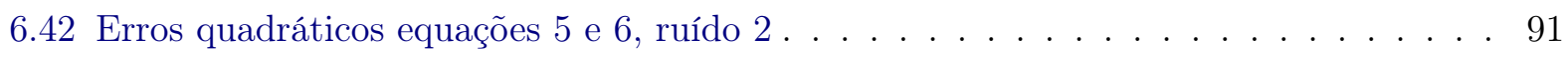

6.43 Erros quadráticos equações 7 e 8 , ruído $2 \ldots \ldots \ldots$. . . . . . . . . . 92

6.44 Erros quadráticos equações 9 e 10a, ruído $2 \ldots \ldots \ldots$. . . . . . . . . 92

6.45 Erros quadráticos equações $10 \mathrm{~b}$ e 11a, ruído $2 \ldots \ldots$. . . . . . . . . 93

6.46 Erros quadráticos equações 11 b e 11c, ruído $2 \ldots \ldots$. . . . . . . . . . 93

6.47 Erros quadráticos equações $12 \mathrm{a}$ e $12 \mathrm{~b}$, ruído $2 \ldots \ldots$. . . . . . . . . 94

6.48 Erros quadráticos equações 12 c, ruído $2 \ldots \ldots \ldots$. . . . . . . . . . 94

6.49 Erros quadráticos equações 1 e 2 , ruído $3 \ldots \ldots \ldots$. . . . . . . . . 95

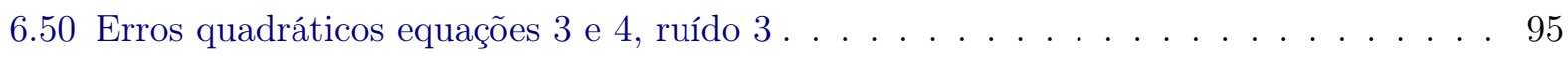

6.51 Erros quadráticos equações 5 e 6 , ruído $3 \ldots \ldots \ldots$. . . . . . . . . 96

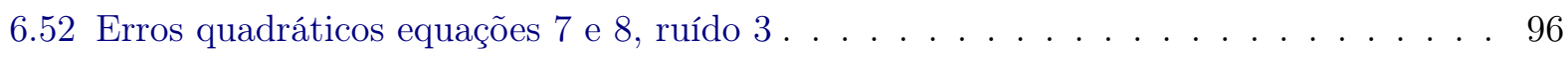

6.53 Erros quadráticos equações 9 e 10a, ruído $3 \ldots \ldots$. . . . . . . . . . . 97

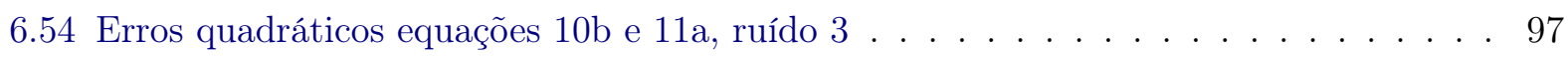

6.55 Erros quadráticos equações 11 b e 11c, ruído $3 \ldots \ldots$. . . . . . . . . . . 98

6.56 Erros quadráticos equações $12 \mathrm{a}$ e $12 \mathrm{~b}$, ruído $3 \ldots \ldots$. . . . . . . . . . . 98

6.57 Erros quadráticos equações 12 c, ruído $3 \ldots \ldots$. . . . . . . . . . . . . 99 


\section{Lista de Tabelas}

6.1 Soluções selecionadas por equação . . . . . . . . . . . . . . . . . . . . . . 44

6.2 Média dos erros quadráticos dos estimadores diretos, sem ruído . . . . . . . . . . . 52

6.3 Média dos erros quadráticos de soluções sem ruído . . . . . . . . . . . . . . . . . . 52

6.4 Média dos erros quadráticos de soluções com ruído $1 \ldots \ldots$. . . . . . . . . . . 52

6.5 Média dos erros quadráticos de soluções com ruído $2 \ldots \ldots$. . . . . . . . . . 53

6.6 Média dos erros quadráticos de soluções com ruído $3 \ldots \ldots$. . . . . . . . . . 53

6.7 Média, sem valores extremos, dos erros quadráticos de soluções com ruído 1 . . . . . 53

6.8 Média, sem valores extremos, dos erros quadráticos de soluções com ruído 2 . . . . 53

6.9 Média, sem valores extremos, dos erros quadráticos de soluções com ruído 3 . . . . . 53

6.10 Erros quadráticos para soluções sem ruído . . . . . . . . . . . . . . . . . . . 54

6.11 Erros quadráticos para soluções sem ruído, estimadores diretos . . . . . . . . . . . 55

6.12 Erros quadráticos para soluções com ruído 1,1 ruído . . . . . . . . . . . . . 56

6.13 Erros quadráticos para soluções com ruído 1, 100 ruídos . . . . . . . . . . . . . . 57

6.14 Erros quadráticos para soluções com ruído 1, 1000 ruídos . . . . . . . . . . . . . 58

6.15 Erros quadráticos para soluções com ruído 2, 1 ruído . . . . . . . . . . . . . . . . . 59

6.16 Erros quadráticos para soluções com ruído 2, 100 ruídos . . . . . . . . . . . . . 60

6.17 Erros quadráticos para soluções com ruído 2, 1000 ruídos . . . . . . . . . . . . . . . 61

6.18 Erros quadráticos para soluções com ruído 3,1 ruído . . . . . . . . . . . . . . . . 62

6.19 Erros quadráticos para soluções com ruído 3,100 ruídos . . . . . . . . . . . . . . . 63

6.20 Erros quadráticos para soluções com ruído 3, 1000 ruídos . . . . . . . . . . . . . . 64 


\section{Capítulo 1}

\section{Introdução}

\subsection{Considerações preliminares}

Equações diferenciais parciais modelam fenômenos da física, engenharia, biologia, química, finanças e outras ciências. Os parâmetros destes problemas são, em muitos casos, os coeficientes que aparecem nas Equação Diferenciais Parciais que modelam esses problemas. Para equações lineares homogêneas é teoricamente possível encontrar esses coeficientes a partir de um grupo de soluções destas EDPs. Numa situação prática na qual desejamos conhecer os coeficientes de uma EDP a partir de observações do fenômeno que ela modela, o que caracteriza um problema inverso, as soluções observadas não seriam exatas, seriam uma aproximação, possivelmente com erros de medição, ou ruídos, das soluções exatas. Desta maneira só conseguiremos uma estimativa dos coeficientes. Assim, necessitamos conhecer o comportamento dos estimadores utilizados quando aplicados a essas aproximações e em relação aos erros de medição, ou ruídos

Neste trabalho vamos analisar os estimadores propostos em [dM11], para os coeficientes funcionais de uma EDP linear homogênea.

\subsection{Objetivos}

O objetivo deste trabalho é analisar, de maneira descritiva utilizando simulações, o desempenho dos estimadores propostos em [dM11]. Para isso selecionaremos um grupo de equações, que são casos particulares da EDP analisada em [dM11], encontraremos as soluções destas equações, e aplicaremos os estimadores a essas soluções. Iremos comparar os coeficientes estimados com os reais, calculando o erro quadrático. Como supomos que as soluções podem ter ruídos, iremos inserir ruídos nas soluções encontradas e analisar o comportamento dos estimadores. Também iremos comparar os estimadores propostos, em [dM11], com os estimadores diretos, mais simples.

\subsection{Organização do trabalho}

Este trabalho está organizado da seguinte maneira:

No capítulo 2 fazemos uma breve apresentação sobre equações diferenciais parciais, discutindo as classificações das EDPs e alguns métodos analíticos de solução. 
No capítulo 3 discutimos alguns métodos de solução numérica para EDPs.

No capítulo 4 descrevemos três algorítimos para gerar ruídos. Esse ruídos serão usados nas simulações, e faremos uma comparação entre eles.

No capítulo 5 apresentamos os estimadores propostos em [dM11], que serão analisados nesse trabalho.

No capítulo 6 faremos a análise e comparação dos estimadores. Iniciamos mostrando as EDPs selecionadas para aplicar os estimadores. Em seguida descrevemos o procedimento utilizado na implementação dos estimadores. Por último apresentamos os resultados das simulações e fazemos a análise dos resultados.

No capítulo 7 apresentamos as conclusões do trabalho. 


\section{Capítulo 2}

\section{Equações Diferenciais Parciais}

Equações diferenciais parciais surgem da modelagem de problemas da física, engenharia, biologia, química, finanças e outras ciências. Esses problemas indicam as propriedades que uma solução deve ter e as condições adicionais impostas à equação.

Normalmente se busca soluções explícitas para a EDP, o que muitas vezes não é possível. Com métodos numéricos é possível encontrar soluções para a maioria das equações. Nem sempre existe solução para uma EDP e as condições adicionais associadas. Assim, saber quando existe solução é uma questão importante no estudos de EDP.

Será dada ênfase as equações lineares envolvendo duas variáveis, visto que a equação, em estudo nesse trabalho, tem essas características.

\subsection{Definição e classificação}

Uma equação diferencial parcial descreve a relação entre variáveis independentes $\left(x_{1}, x_{1}, \ldots, x_{n}\right)$, uma função desconhecida destas variáveis $\left(u\left(x_{1}, x_{1}, \ldots, x_{n}\right)\right)$, e suas derivadas parciais. Usando a notação $u_{x_{i}}=\partial u / \partial x_{i}$, a forma geral de uma EDP é

$$
F\left(x_{1}, x_{1}, \cdots, x_{n}, u, u_{x_{1}}, u_{x_{2}}, \cdots, u_{x_{1} x_{1}}, \cdots\right)=0
$$

Podemos classificar uma EDP de diversas maneiras, pela ordem de suas derivadas, se é linear ou não, e se é homogênea ou não, estas são as classificações mais comuns. Veremos depois as classificações das equações de segunda ordem.

A ordem de uma EDP é a ordem da maior derivada na equação. Assim, uma equação de segunda ordem apresenta pelo menos uma derivada de segunda ordem.

Para classificarmos uma EDP quanto a linearidade precisamos do conceito de operador diferencial. Uma EDP pode ser escrita na forma $L(u)=g$, onde $L$ é um operador diferencial e $g$ uma função nas variáveis independentes. Com exemplo temos: 


$$
\begin{array}{ll}
u_{x}+u_{y}=0 \rightarrow & L=\frac{\partial}{\partial x}+\frac{\partial}{\partial y}, \\
u_{x}+y u_{y}=0 \rightarrow \quad L=\frac{\partial}{\partial x}+y \frac{\partial}{\partial y}, \\
u_{x x}+u_{y y}=0 \rightarrow \quad L=\frac{\partial^{2}}{\partial x^{2}}+\frac{\partial^{2}}{\partial y^{2}} .
\end{array}
$$

Se $L$ é um operador linear, isto é,

$$
\begin{aligned}
& L(u+v)=L(u)+L(v), \\
& L(k u)=k L(u),
\end{aligned}
$$

dizemos que a equação é linear, caso contrário não linear. As equações não lineares podem ser classificadas em semilinear, quasilinear, e totalmente não linear. Se a equação é linear nos termos que contém derivadas, e não linear nos termos que envolvem apenas $u$, dizemos então que a equação é semilinear. Se as derivadas de ordem igual a ordem da equação formam uma expressão linear dizemos que a EDP é quasilinear. Caso a equação não seja linear, semilinear ou quasilinear, dizemos que é totalmente não linear.

Escrevendo a equação na seguinte forma $L(u)=g$, se $g$ é identicamente nula $(g \equiv 0)$, dizemos que a equação é homogênea, caso contrário não homogênea. Se $u_{1}, u_{2}, \cdots, u_{n}$ são soluções de uma equação linear homogênea $(L(u)=0)$, então qualquer combinação linear de $u_{1}, u_{2}, \cdots, u_{n}$ $\left(v=\sum_{i=1}^{n} \alpha_{i} u_{i}\right)$, é também uma solução da equação. Isto é chamado de princípio da superposição.

Como exemplo de equações diferenciais parciais temos:

Equação do calor da física

Equação da onda da física.

Equação de Navier-Stokes da mecânica dos fluídos.

Equação Schrödinger da mecânica quântica.

Equação de Black-Sholes de finanças

$$
\begin{aligned}
& u_{t}-k u_{x x}=0 \\
& u_{t t}-c^{2} u_{x x}=\frac{1}{\rho} f(x, t)
\end{aligned}
$$

$\rho\left(\frac{\partial \mathbf{v}}{\partial t}+\mathbf{v} \cdot \nabla \mathbf{v}\right)=-\nabla p+\nabla \cdot \mathbf{T}+\mathbf{f}$

$$
i \hbar \frac{\partial}{\partial t} \Psi=\left(-\frac{\hbar^{2}}{2 m} \Delta-V\right) \Psi
$$

$$
\frac{1}{2} \sigma^{2} S^{2} \frac{\partial^{2} V}{\partial S^{2}}+r S \frac{\partial V}{\partial S}+\frac{\partial V}{\partial t}-r V=0
$$

\subsection{Condições iniciais e de contorno}

Uma equação diferencial parcial sem nenhuma condição adicional, quando tem solução, normalmente tem infinitas soluções. Por exemplo, na EDP $u_{x y}=0$. Integrando em relação a $x$ obtemos

$$
u_{y}=f(y)
$$

Integrando em relação a $y$ obtemos

$$
u=F(y)+G(x)
$$


Assim, para qualquer par de funções $F$ e $G$, onde $F^{\prime}=f, u$ é uma solução da equação. Para que exista uma única solução, condições adicionais devem ser impostas. Essas condições são, normalmente, definidas pelo problema modelado pela equação. Essas condições podem ser basicamente de dois tipos, condições iniciais e condições de contorno. Condições iniciais especificam o comportamento da solução em um tempo inicial $t_{0}$, ou especificando uma curva que a solução teve conter. Por exemplo.

$$
\begin{gathered}
u\left(x, t_{0}\right)=f(x), \\
u_{t}\left(x, t_{0}\right)=g(x) .
\end{gathered}
$$

Condições de contorno especificam o comportamento da solução na borda do domínio, onde se deseja encontrar a solução. Por exemplo

$$
u_{t}=k \nabla u, \quad(x, y, z) \in \partial \Omega,
$$

onde $\partial \Omega$ é a borda do domínio. Existem, basicamente, três tipos de condições de contorno. Condição de Dirichlet, quando os valores da solução, na borda, são especificados.

$$
u(x, y, z, t)=h(x, y, z, t), \quad(x, y, z) \in \partial \Omega .
$$

Condição de Neumann, quando o comportamento da derivada normal na borda é especificado.

$$
\partial_{n} u(x, y, z, t)=k(x, y, z, t), \quad(x, y, z) \in \partial \Omega .
$$

Condição de Robin, quando uma relação entre os valores de $u$ e a derivada normal, na borda, é especificado.

$$
a(x, y, z) \partial_{n} u(x, y, z, t)+u(x, y, z, t)=k(x, y, z, t), \quad(x, y, z) \in \partial \Omega .
$$

Outras combinações de condições são possíveis.

\subsection{Problema bem-posto}

Saber quando uma EDP, junto com suas condições iniciais e/ou de contorno, têm solução e é única, é uma questão importante na teoria de equações diferenciais parciais. Se condições suficientes não são impostas, o problema pode ter mais de uma solução. Se condições em excesso são impostas ou as condições são incompatíveis com a equação, pode não haver solução. Normalmente as condições impostas são originadas do fenômeno que a EDP está modelando, mas isso não garante que a equação terá solução. Outro problema é a estabilidade da solução, ou seja, pequenas variações nos parâmetros e/ou condições adicionais, devem gerar pequenas variações na solução. Em problemas envolvendo fenômenos reais os parâmetros da equação e/ou condições adicionais são determinados por medições que têm uma precisão limitada. Assim, se a solução não for estável, qualquer variação na medição dos parâmetros pode levar a uma variação muito grande na solução, tornando o problema instável. 
Um problema bem-posto, como definido pelo matemático Jacques Hadamard, têm as seguintes propriedades.

1. Existência. Existe pelo menos uma solução para o problema.

2. Unicidade. A solução é única.

3. Estabilidade. Uma pequena mudança nos parâmetros da equação ou condições, leva a uma pequena mudança na solução.

\subsection{Equações de primeira ordem}

Uma equação envolvendo duas variáveis independentes de primeira ordem têm a seguinte forma geral.

$$
F\left(x, y, u, u_{x}, u_{y}\right)=0
$$

No caso de equações lineares e quasilineares temos as seguintes formas gerais:

$$
\begin{gathered}
a(x, y, u) u_{x}+b(x, y, u) u_{y}=c_{0}(x, y) u+c_{1}(x, y), \\
a(x, y) u_{x}+b(x, y) u_{y}=c(x, y, u) .
\end{gathered}
$$

Uma solução $u(x, y)$, da EDP, é uma superfície em $\mathbb{R}^{3}$ e a normal a essa superfície é dada por $\left(u_{x}, u_{y},-1\right)$. Reescrevendo as equações (2.3) e (2.4) temos:

$$
\begin{gathered}
\left(a(x, y, u), b(x, y, u), c_{0}(x, y) u+c_{1}(x, y)\right) \cdot\left(u_{x}, u_{y},-1\right)=0 \\
(a(x, y, u), b(x, y, u), c(x, y, u)) \cdot\left(u_{x}, u_{y},-1\right)=0 .
\end{gathered}
$$

Assim, as equações (2.3) e (2.4) podem ser vistas como o relacionamento entre a superfície a sua normal. Os vetores $\left(a(x, y, u), b(x, y, u), c_{0}(x, y) u+c_{1}(x, y)\right)$ e $(a(x, y, u), b(x, y, u), c(x, y, u))$ pertencem ao plano tangente à superfície.

Uma condição normalmente imposta a uma EDP de primeira ordem, é fornecer uma curva $\Gamma$ que está contida na solução, chamada de curva inicial. Um problema envolvendo uma EDP e uma curva inicial é chamado de problema de Cauchy. Um método de solução de equações lineares ou quasilineares, de primeira ordem, é o método das características. A ideia, deste método, é encontrar curvas, na superfície solução, ao longo das quais a EDP se reduz a uma equação diferencial ordinária. Dado que os vetores $\left(a(x, y, u), b(x, y, u), c_{0}(x, y) u+c_{1}(x, y)\right)$ e $(a(x, y, u), b(x, y, u), c(x, y, u))$ 
pertencem ao plano tangente à superfície, obtemos o seguinte sistema de equações

$$
\begin{aligned}
\frac{d x}{d t}(t) & =a(x(t), y(t)) \\
\frac{d y}{d t}(t) & =a(x(t), y(t)) \\
\frac{d u}{d t}(t) & =c_{0}(x(t), y(t)) u+c_{1}(x(t), y(t))
\end{aligned}
$$

que define as curvas características para uma EDP linear de primeira ordem. No caso de uma EDP quasilinear temos o seguinte sistema

$$
\begin{aligned}
& \frac{d x}{d t}(t)=a(x(t), y(t), u(t)) \\
& \frac{d y}{d t}(t)=b(x(t), y(t), u(t)) \\
& \frac{d u}{d t}(t)=c(x(t), y(t), u(t)) .
\end{aligned}
$$

Estes sistemas, de EDOs, são chamados de sistemas de equações características, ou simplesmente, equações características. As soluções destes sistemas são as curvas características. Para determinar a curva característica precisamos de uma condição inicial. Tomando como condição inicial um ponto $\Gamma(s)$ da curva inicial. Escrevendo $\Gamma$ numa forma paramétrica

$$
\Gamma=\Gamma(s)=\left(x_{0}(s), y_{0}(s), u_{0}(s)\right)
$$

e levando em consideração que cada curva característica $(x(t), y(t), u(t))$ começa em um ponto $s$ diferente na curva inicial, vamos escrever a curva na forma $(x(t, s), y(t, s), u(t, s))$ e a condição inicial como

$$
x(0, s)=x_{0}(s), y(0, s)=y_{0}(s), u(0, s)=u_{0}(s)
$$

Nesse caso consideramos $t=0$ para o ponto da curva característica sobre a curva inicial. Observe que, em geral, a parametrização $(x(t, s), y(t, s), u(t, s))$ representa uma superfície no $\mathbb{R}^{3}$. Assim, essa superfície representa a união das curvas características obtidas para cada ponto da curva inicial. A superfície $(x(t, s), y(t, s), u(t, s))$ é chamada de superfície integral. Para obtermos a solução $u(x, y)$ é necessário inverter $x(t, s)$ e $y(t, s)$. Isso é possível quando o Jacobiano $J=$ $\partial(x, y) / \partial(t, s) \neq 0$. Calculando o Jacobiano ao longo da curva inicial temos

$$
J=\frac{\partial x}{\partial t} \frac{\partial y}{\partial s}-\frac{\partial x}{\partial s} \frac{\partial y}{\partial t}=\left|\begin{array}{cc}
a & b \\
\left(x_{0}\right)_{s} & \left(y_{0}\right)_{s}
\end{array}\right|=a\left(y_{0}\right)_{s}-b\left(x_{0}\right)_{s},
$$

onde $\left(x_{0}\right)_{s}=s x_{0} / d s$. Se $J \neq 0$ para os pontos da curva inicial, então as EDPs (2.3) e (2.4) têm uma a única solução perto da curva inicial. Esta condição é chamada de condição de transversalidade. A condição de transversalidade têm uma interpretação geométrica. Analisando a expressão (2.8) vemos que $J=0$ quando os vetores $(a, b)$ e $\left(\left(x_{0}\right)_{s},\left(y_{0}\right)_{s}\right)$ são linearmente dependentes. Isso significa que a projeção da curva inicial e da curva característica, no plano $(x, y)$, são tangentes nesse ponto.

De maneira precisa podemos dizer que se os coeficientes das equações (2.3) e (2.4) são funções suaves na vizinhança da curva inicial, e que se a condição de transversalidade é satisfeita para 
todo ponto $s$ no intervalo $\left(s_{0}-2 \delta, s+0+2 \delta\right)$ na curva inicial, então os problemas, representados pelas equações (2.3) e (2.4) mais uma curva inicial, têm uma única solução na vizinhança $(t, s) \in$ $(-\epsilon, \epsilon) \times\left(s_{0}-\delta, s+0+\delta\right)$ da curva inicial. Caso contrário não existe solução ou existem infinitas soluções.

\subsection{Equações de segunda ordem}

As equações lineares de segunda ordem para funções de duas variáveis independentes $x, y$ têm a seguinte forma

$$
L[u]=a u_{x x}+2 b u_{x y}+c u_{y y}+d u_{x}+e u_{y}+f u=g,
$$

onde $a, b, c, d, e, f, g$ são funções de $(x, y), a, b, c$ não são nulas simultaneamente e $u$ é uma função desconhecida. O termo $a u_{x x}+2 b u_{x y}+c u_{y y}$, composto pelos termos de segunda ordem, é chamado de parte principal da equação. Dado um ponto $(x, y)$ dizemos que a equação é

- Hiperbólica se $b(x, y)^{2}-a(x, y) c(x, y)>0$

- Parabólica se $b(x, y)^{2}-a(x, y) c(x, y)=0$

- Elíptica se $b(x, y)^{2}-a(x, y) c(x, y)<0$.

Dado um domínio $\Omega$ no $\mathbb{R}^{2}$, dizemos que a equação é hiperbólica em $\Omega$ se ela é hiperbólica em todos os ponto de $\Omega$. Da mesma maneira classificamos como parabólica e elíptica. As principais características e métodos de solução são determinados pelo tipo da equação. É possível mostrar que o tipo de uma EDP de segunda ordem não se altera por uma mudança de coordenadas qualquer $(\xi, \eta)=(\xi(x, y), \eta(x, y))$.

Dado um domínio $\Omega$, onde a EDP seja de um tipo especifico, existe um sistema de coordenadas em que a EDP tenha uma forma mais simples, chamada de forma canônica.

- Se for hiperbólica em $\Omega$ então a forma canônica é $\omega_{\xi \eta}+l_{1}[\omega]=G(\xi, \eta)$

- Se for parabólica em $\Omega$ então a forma canônica é $\omega_{\xi \xi}+l_{1}[\omega]=G(\xi, \eta)$

- Se for elíptica em $\Omega$ então a forma canônica é $\omega_{\xi \xi}+\omega_{\eta \eta}+l_{1}[\omega]=G(\xi, \eta)$,

onde $\omega(\xi, \eta)=u(x(\xi, \eta), y(\xi, \eta)), l_{1}$ é um operador diferencial linear de primeira ordem e $G$ é um função. No caso de a EDP ser elíptica é necessário impor outras restrições, domínio $\Omega$ deve ser planar e as funções $a, b, c$ devem ser analíticas em $\Omega$. 


\subsubsection{Equação da onda}

A equação da onda é uma tipica equação hiperbólica. A equação da onda homogênea, em uma dimensão, têm a seguinte forma

$$
u_{t t}-c^{2} u_{x x}=0
$$

onde $-\infty \leq a<x<b \leq \infty, t>0$ e $c \in \mathbb{R}$. Esta é uma equação hiperbólica. Podemos obter a forma canônica fazendo a seguinte mudança de coordenadas.

$$
\xi=x+c t, \quad \eta=x-c t .
$$

Aplicando a mudança de coordenadas obtemos

$$
u_{t t}-c^{2} u_{x x}=-4 c^{2} w_{\xi \eta}=0 .
$$

A solução geral desta equação é

$$
w(\xi, \eta)=F(\xi)+G(\eta) .
$$

Voltando para as coordenadas originais a solução fica

$$
u(x, t)=F(x+c t)+G(x-c t) .
$$

Com as condições iniciais, normalmente aplicadas para a equação da onda homogênea, obtemos o problema de Cauchy 2.11:

$$
\begin{aligned}
& u_{t t}-c^{2} u_{x x}=0, \quad-\infty<x<\infty t>0, \\
& u(x, 0)=f(x), \quad u_{t}(x, 0)=g(x), \quad-\infty<x<\infty .
\end{aligned}
$$

Aplicando as condições iniciais à solução geral (2.10), obtemos a solução desse problema, que é chamada de fórmula de d'Alembert.

$$
u(x, t)=\frac{f(x+c t)+f(x-c t)}{2}+\frac{1}{2 c} \int_{x-c t}^{x+c t} g(s) d s .
$$

No caso da equação da onda não homogênea, o problema de Cauchy fica

$$
\begin{aligned}
& u_{t t}-c^{2} u_{x x}=F(x, t), \quad-\infty<x<\infty t>0, \\
& u(x, 0)=f(x), \quad u_{t}(x, 0)=g(x), \quad-\infty<x<\infty .
\end{aligned}
$$

Fazendo uso da fórmula de Green

$$
\iint_{\Omega}\left[Q(x, t)_{x}-P(x, t)_{t}\right] d x d t=\oint_{\Gamma}[Q(x, t) d x+P(x, t) d t]
$$


obtemos a solução dada pela seguinte fórmula de d'Alembert

$$
u(x, t)=\frac{f(x+c t)+f(x-c t)}{2}+\frac{1}{2 c} \int_{x-c t}^{x+c t} g(s) d s+\frac{1}{2 c} \int_{0}^{t} \int_{x-c(t-\tau)}^{x-c(t+\tau)} F(\xi, \tau) d \xi d \tau
$$

Dado o domínio $-\infty<x<\infty, 0 \leq t \leq T$ e supondo $f \in C^{2}, g \in C^{1}$ ( $C^{n}$ indica que a enésima derivada da função é continua), é possível mostrar que o problema de Cauchy (2.11) é um problema bem-posto. Acrescentando a condição $F, F_{x} \in C^{2}$ também é possível mostrar que o problema de Cauchy (2.13) é bem-posto.

\subsubsection{Método da separação de variáveis}

Embora esse método seja aplicado para resolver muitos problemas lineares importantes, ele não pode ser aplicado para qualquer EDP linear. O método pode ser aplicado em problemas onde a equação e o domínio têm uma simetria apropriada. Na maioria dos casos o domínio deve ser limitado. O método é composto, basicamente, pelos seguintes passos.

- Supomos que a solução têm a seguinte forma, $u(x, t)=X(x) T(t)$.

- Substituímos, a solução procurada, na equação e separamos as variáveis obtendo $F_{1}\left(x, X, X_{x}, X_{x x}\right)=$ $F_{2}\left(t, T, T_{t}, T_{t t}\right)$, que devem ser iguais a uma constante $-\lambda$. E assim obtemos duas EDOs, $F_{1}\left(x, X, X_{x}, X_{x x}\right)=-\lambda, \quad F_{2}\left(t, T, T_{t}, T_{t t}\right)=-\lambda$.

- Procuramos soluções para a primeira equação, que satisfaça as condições de contorno/iniciais. E assim obtemos os autovalores $\lambda_{n}$ e as autofunções $X_{n}(x)$ associadas.

- Procuramos soluções para a segunda equação $T_{n}$ associadas a $\lambda_{n}$ na seguinte forma $T_{n}=$ $A_{n} T_{n 1}+B_{n} T_{n 2}$.

- Aplicamos o princípio da superposição e obtemos uma solução da forma $u(x, t)=X_{1}(x) T_{1}(t)+$ $X_{2}(x) T_{2}(t)+\cdots$

- Calculamos os coeficientes $A_{n}$ e $B_{n}$ utilizando as condições de contorno/iniciais.

Vamos exemplificar o método para equações homogêneas do calor e da onda. Vamos analisar, primeiramente, um problema envolvendo a equação do calor.

$$
\begin{aligned}
& u_{t}-k u_{x x}=0 \quad 0<x<L t>0 \\
& u(0, t)=u(L, t)=0 \quad t>0, \\
& u(x, 0)=f(x),
\end{aligned}
$$


onde $f$ é uma condição inicial e $k$ é uma constante positiva. Também é aplicada a seguinte condição de compatibilidade $f(0)=f(L)=0$. Procuramos soluções da seguinte forma

$$
u(x, t)=X(x) T(t)
$$

Derivando a expressão (2.16) em relação a $t$ e duas vezes em relação a $x$, e substituindo na EDP, obtemos

$$
\frac{T_{t}}{k T}=\frac{X_{x x}}{X}=-\lambda
$$

Isso nos leva ao seguinte sistema de EDOs

$$
\begin{gathered}
\frac{d^{2} X}{d x^{2}}=-\lambda X \quad 0<x<L \\
\frac{d T}{d t}=-\lambda k T \quad t>0 .
\end{gathered}
$$

Da teoria de EDO sabemos que as possíveis soluções deste sistema dependem do valor de $\lambda$, isto é, $\lambda<0, \lambda=0$ ou $\lambda>0$. Analisando os valores de $\lambda$ para os quais as equações (2.17) e (2.18) têm soluções não triviais, obtemos o seguinte conjunto de valores para $\lambda$.

$$
\lambda=\left(\frac{n \pi}{L}\right)^{2} \quad n=1,2,3, \cdots
$$

As soluções das EDOs associadas a esses valores são

$$
\begin{aligned}
& X_{n}(x)=\sin \left(\frac{n \pi x}{L}\right) \quad n=1,2,3, \cdots \\
& T_{n}(t)=B_{n} e^{-k\left(\frac{n \pi}{L}\right)^{2} t} \quad n=1,2,3, \cdots
\end{aligned}
$$

Assim, obtemos as seguintes soluções para o problema (2.15)

$$
u_{n}(x, t)=B_{n} \sin \left(\frac{n \pi x}{L}\right) e^{-k\left(\frac{n \pi}{L}\right)^{2} t} \quad n=1,2,3, \cdots
$$

Para uma solução mais geral aplicamos o princípio da superposição generalizado, que é a aplicação do princípio da superposição para séries infinitas. Assim, obtemos a seguinte solução generalizada.

$$
u(x, t)=\sum_{n=1}^{\infty} B_{n} \sin \left(\frac{n \pi x}{L}\right) e^{-k\left(\frac{n \pi}{L}\right)^{2} t},
$$

onde os coeficientes $B_{n}$ são determinados pela seguinte expressão:

$$
B_{n}=\frac{2}{L} \int_{0}^{L} \sin \frac{n \pi x}{L} f(x) d x \quad n=1,2,3, \cdots
$$


Vamos agora aplicar o método da separação de variáveis à equação da onda. Vamos considerar o seguinte problema

$$
\begin{aligned}
& u_{t t}-c^{2} u_{x x}=0, \quad 0<x<L, t>0 \\
& u_{x}(0, t)=u_{x}(L, t)=0 \quad t \geq 0 \\
& u(x, 0)=f(x)=0, \quad 0 \leq x \leq L \\
& u_{t}(x, 0)=g(x)=0, \quad 0 \leq x \leq L
\end{aligned}
$$

Onde $f$ e $g$ são funções e $c$ é uma constate positiva. As condições de compatibilidade são $f^{\prime}(0)=$ $f^{\prime}(L)=g^{\prime}(0)=g^{\prime}(L)=0$. Supondo que a solução tenha o mesmo formato, $u(x, t)=X(x) T(t)$ obtemos

$$
\frac{T_{t t}}{c^{2} T}=\frac{X_{x x}}{X}=-\lambda
$$

Isso nos leva ao seguinte sistema de EDOs

$$
\begin{gathered}
\frac{d^{2} X}{d x^{2}}=-\lambda X, \quad 0<x<L \\
\frac{d^{2} T}{d t^{2}}=-\lambda c 2 T, \quad t>0 .
\end{gathered}
$$

Analisando os valores de $\lambda$ para os quais as equações (2.23) e (2.24) têm solução, obtemos, novamente, a expressão (2.19). Aplicando o mesmo raciocínio anterior obtemos a seguinte solução para o problema $(2.22)$

$$
u(x, t)=\frac{A_{0}+B_{0} t}{2}+\sum_{n=1}^{\infty}\left(A_{n} \cos \frac{c \pi n t}{L}+B_{n} \sin \frac{c \pi n t}{L}\right) \cos \frac{n \pi x}{L} .
$$

os coeficientes são dados por

$$
\begin{gathered}
A_{0}=\frac{2}{L} \int_{0}^{L} f(x) d x \\
A_{n}=\frac{2}{L} \int_{0}^{L} \cos \frac{n \pi x}{L} f(x) d x \quad n=1,2,3, \cdots \\
B_{0}=\frac{2}{L} \int_{0}^{L} g(x) d x \\
B_{n}=\frac{2}{c \pi n} \int_{0}^{L} \cos \frac{n \pi x}{L} g(x) d x \quad n=1,2,3, \cdots
\end{gathered}
$$

Uma adaptação do método de separação de variáveis pode ser aplicado às equações não homogêneas, é chamado de expansão em autofunções. 


\subsubsection{Equações elípticas}

Uma típica equação elíptica é a equação de Laplace.

$$
\Delta u=u_{x x}+u_{y y}=0 .
$$

O método de solução, mais simples, para equações de Laplace é o método da separação de variáveis. Mas ele só pode ser aplicado em domínios simples como retângulos, discos, etc. Vamos analisar, como exemplo, dois problemas, o primeiro em um domínio retangular e o segundo em um domínio circular.

Seja o seguinte problema, em um domínio retangular

$$
\begin{aligned}
& u_{x x}+u_{y y}=0 \quad a<x<b, c<y<d \\
& u(a, y)=f(y) \\
& u(b, y)=g(y) \\
& u(x, c)=h(x) \\
& u(x, d)=k(x) .
\end{aligned}
$$

Vamos dividir a solução $u$ da seguinte maneira

$$
u=u_{1}+u_{2}
$$

onde $u_{1}$ e $u_{2}$ satisfazem as seguintes condições de contorno

$$
\begin{aligned}
& u_{1}(a, y)=f(y) \\
& u_{1}(b, y)=g(y) \\
& u_{1}(x, c)=0 \\
& u_{1}(x, d)=0 \\
& u_{2}(a, y)=0 \\
& u_{2}(b, y)=0 \\
& u_{2}(x, c)=h(x) \\
& u_{2}(x, d)=g(x) .
\end{aligned}
$$

E impomos a seguinte condição de compatibilidade

$$
f(c)=f(d)=g(c)=g(d)=h(a)=h(b)=k(a)=k(b)=0
$$

Assim, dividimos nosso problema em dois, que podem ser resolvidos separadamente, obtendo

$$
\begin{aligned}
& u_{1}(x, y)=\sum_{n} X_{1 n}(x) Y_{1 n}(y) \\
& u_{2}(x, y)=\sum_{n} X_{2 n}(x) Y_{2 n}(y) .
\end{aligned}
$$


Vamos analisar, agora, um problema em um domínio circular. Seja $B_{a}$ um disco de raio $a$ com centro na origem do sistema de coordenadas, e o seguinte problema:

$$
\begin{aligned}
& u_{x x}+u_{y y}=0 \quad(x, y) \in B_{a} \\
& u(x, y)=g(x, y) \quad(x, y) \in \partial B_{a} .
\end{aligned}
$$

Alterando o sistema de coordenadas para coordenadas polares, a solução procurada é $w(r, \theta)=$ $u(x(r, \theta), y(r, \theta))$, e o passa a ser

$$
w_{r r}+\frac{1}{r} w_{r}+\frac{1}{r^{2}} w_{\theta \theta}=0 \quad 0<r<a, 0<\theta<2 \pi w(a, \theta)=h(\theta) .
$$

Onde $h(\theta)=g(x(a, \theta), y(a, \theta))$. A solução procurada têm a seguinte forma $w(r, \theta)=R(r) \Theta(\theta)$, e assim obtemos o seguinte sistema de EDOs.

$$
\begin{aligned}
& r^{2} R^{\prime \prime}(r)+r R^{\prime}(r)+\lambda R(r)=0 \quad 0<r<a \\
& \Theta^{\prime \prime}(\theta)+\lambda \Theta(\theta)=0 \quad 0<\theta<2 \pi .
\end{aligned}
$$

Devido ao domínio, original ser circular, é necessário impor também as seguintes condições de periodicidade.

$$
\begin{aligned}
& \Theta(0)=\Theta(2 \pi) \\
& \Theta^{\prime}(0)=\Theta^{\prime}(2 \pi) .
\end{aligned}
$$

Como solução obtemos

$$
w(r, \theta)=\frac{\alpha_{0}}{2}+\sum_{n=1}^{\infty} r^{n}\left(\alpha_{n} \cos (n \theta)+\beta_{n} \sin (n \theta)\right),
$$

onde

$$
\begin{aligned}
\alpha_{0} & =\frac{1}{\pi} \int_{0}^{2 \pi} h(\varphi) d \varphi \\
\alpha_{n} & =\frac{1}{\pi a^{n}} \int_{0}^{2 \pi} h(\varphi) \cos (n \varphi) d \varphi \\
\beta_{n} & =\frac{1}{\pi a^{n}} \int_{0}^{2 \pi} h(\varphi) \sin (n \varphi) d \varphi .
\end{aligned}
$$

Existem outros métodos de solução de EDPs como a transformada de Laplace, a transformada de Fourier e outros. A escolha do método depende do tipo da EDP de segundo grau. Este capítulo foi baseado nos livro [PR05], [Str08] e [Pol02]. 


\section{Capítulo 3}

\section{Métodos Numéricos para Equações Diferenciais Parciais}

Encontrar uma solução analítica para uma equação diferencial parcial não é possível, na maioria dos casos. Os métodos analíticos estão limitados a equações de formas canônicas em domínios simples. Equações não lineares, com coeficientes não constantes ou em domínios complexos, normalmente não tem soluções analíticas. Os métodos numéricos são uma forma de encontrar soluções aproximadas para equações mais complexas e em domínios mais complexos.

A ideia básica dos métodos numéricos é discretizar o problema, substituindo as variáveis contínuas por variáveis discretas. Desta maneira, a EDP é substituída por um sistema de equações algébricas. Assim, a solução aproximada obtida é um conjunto de valores discretos que as variáveis assumem. A precisão da solução depende do número de variáveis discretas ou equações algébricas utilizadas. Assim, uma boa aproximação necessita de um número grande de equações algébricas.

Os métodos numéricos mais comuns são o método de diferenças finitas e o método de elementos finitos. O método de diferenças finitas é mais simples de implementar. O método de elementos finitos apresenta uma dificuldade maior, embora seja mais flexível para problemas envolvendo geometrias complexa. Neste trabalho utilizamos o método das linhas para encontrar soluções numéricas.

Nesse capítulo faremos uma breve descrição do método de diferenças finitas, tendo como base [PR05] e [Tho95], e do método das linhas, descrito em [Sch91] e [SG09].

\subsection{Método de diferenças finitas}

O método de diferenças finitas é baseado na substituição das derivadas, na equação diferencial parcial, por aproximações de diferenças finitas. Para isso é necessário discretizar o domínio da solução. Supondo um domínio retangular $D=[a, b] \times[c, d]$ obtemos um grade de pontos in $D$ da seguinte maneira:

$$
\left(x_{i}, y_{j}\right)=(i \Delta x, j \Delta y) \quad 0 \leq i \leq N-1, \quad 0 \leq j \leq M-1,
$$

onde $\Delta x=(b-a) /(N-1)$ e $\Delta y=(d-c) /(M-1)$. Os valores que $u$ assume na grade de pontos 
é representado por $U_{i, j}=u\left(x_{i}, y_{j}\right)$. Para obter as aproximações das derivadas é usada a expansão por série de Taylor de $u$ entorno do ponto $\left(x_{i}, y_{j}\right)$.

$$
u\left(x_{i+1}, y_{j}\right)=u\left(x_{i}, y_{j}\right)+\partial_{x}\left(x_{i}, y_{j}\right) \Delta x+\frac{1}{2} \partial_{x}^{2}\left(x_{i}, y_{j}\right)(\Delta x)^{2}+\frac{1}{6} \partial_{x}^{3}\left(x_{i}, y_{j}\right)(\Delta x)^{3}+\cdots
$$

Usando (3.1) é possível obter as seguintes aproximações para as derivadas de primeira de segunda ordem.

$$
\begin{aligned}
& \partial_{x}\left(x_{i}, y_{j}\right) \sim \frac{U_{i+1, j}-U_{i, j}}{\Delta x}, \\
& \partial_{x}\left(x_{i}, y_{j}\right) \sim \frac{U_{i, j}-U_{i-1, j}}{\Delta x}, \\
& \partial_{x}\left(x_{i}, y_{j}\right) \sim \frac{U_{i+1, j}-U_{i-1, j}}{2 \Delta x}, \\
& \partial_{y}\left(x_{i}, y_{j}\right) \sim \frac{U_{i, j+1}-U_{i, j}}{\Delta y}, \\
& \partial_{x}\left(x_{i}, y_{j}\right) \sim \frac{U_{i, j}-U_{i, j-1}}{\Delta y}, \\
& \partial_{y}\left(x_{i}, y_{j}\right) \sim \frac{U_{i, j+1}-U_{i, j-1}}{2 \Delta y}, \\
& \partial_{x}\left(x_{i}, y_{j}\right) \sim \frac{U_{i-1, j}-2 U_{i, j}+U_{i+1, j}}{(\Delta x)^{2}}, \\
& \partial_{y}\left(x_{i}, y_{j}\right) \sim \frac{U_{i, j-1}-2 U_{i, j}+U_{i, j+1}}{(\Delta y)^{2}} .
\end{aligned}
$$

As expressões (3.2) e (3.5) são chamadas de diferença finita progressiva, as (3.3) e (3.6) são chamadas de diferença finita regressiva e as (3.4), (3.7), (3.8) e (3.9) diferença finita centrada. Outras aproximações são possíveis de serem obtidas. Quando substituímos as derivadas, de uma EDP, por aproximações obtemos um esquema numérico para discretizar a EDP. Tomando a equação do calor como exemplo

$$
\begin{array}{ll}
u_{t}=k u_{x x}, & 0<x<\pi, t>0 \\
u(0, t)=u(\pi, t)=0, & t \geq 0 \\
u(x, 0)=u(0), & 0 \leq x \leq \pi .
\end{array}
$$


Para discretizar o problema vamos tomar $\Delta t=T /(M-1)$ e $\Delta x=\pi /(N-1)$, assim, $U_{i, n}=$ $u(i \Delta x, n \Delta t)$. Substituindo as derivadas da EDP pelas expressões (3.5) e (3.8) obtemos

$$
\begin{array}{ll}
\frac{U_{i, n+1}-U_{i, n}}{\Delta t}=k \frac{U_{i+1, n}-2 U_{i, n}+U_{i-1, n}}{(\Delta x)^{2}}, & 1 \leq i \leq N-2,0 \leq n \leq M-2 \\
U_{0, n}=U_{N-1, n}=0, & 0 \leq n \leq M-2 \\
U_{i, 0}=f(i \Delta x), & 1 \leq i \leq N-2 .
\end{array}
$$

Este é um esquema numérico simples e que não é estável dependendo da escolha de $\Delta t$. Uma pequena alteração nas condições iniciais leva a uma alteração grande na solução. Assim, vemos que um esquema deve satisfazer certas condições para ser aplicável. Ele deve ser estável, pequenas alterações nas condições do problema levam a pequenas alterações na solução. Deve ser consistente, a solução da EDP deve satisfazer o esquema quando $\Delta x \rightarrow 0$ e $\Delta t \rightarrow 0$. Deve ser convergente, a solução do problema discreto converge para a solução da EDP quando $\Delta x \rightarrow 0$ e $\Delta t \rightarrow 0$. É possível mostrar que um esquema numérico estável e consistente é convergente. Para o esquema (3.11) ser estável devemos $\Delta t$ muito pequeno. O que torna o esquema pouco eficiente. Como exemplo de esquemas estáveis e consistentes para o problema (3.10) temos o esquema de Crank e Nicolson

$$
\frac{U_{i, n+1}-U_{i, n}}{\Delta t}=k\left(\frac{U_{i+1, n}-2 U_{i, n}+U_{i-1, n}}{2(\Delta x)^{2}}+\frac{U_{i+1, n+1}-2 U_{i, n+1}+U_{i-1, n+1}}{2(\Delta x)^{2}}\right)
$$

e o esquema de Du-Fort e Frankel

$$
\frac{U_{i, n+1}-U_{i, n-1}}{\Delta t}=k \frac{U_{i+1, n}-U_{i, n-1}-U_{i, n+1}+U_{i-1, n}}{2(\Delta x)^{2}}
$$

Um exemplo de equação elíptica é a equação de Laplace.

$$
\begin{array}{ll}
u_{x x}(x, y)+u_{y y}(x, y)=f(x, y) & (x, y) \in \Omega=(0, a) \times(0, b) \\
u(x, y)=g(x, y) & (x, y) \in \partial \Omega
\end{array}
$$

Um possível esquema numérico para este problema é

$$
\begin{array}{ll}
\frac{U_{i-1, j}-2 U_{i, j}+U_{i+1, j}}{(\Delta x)^{2}}+\frac{U_{i, j-1}-2 U_{i, j}+U_{i, j+1}}{(\Delta y)^{2}}=f(i \Delta x, j \Delta y) & i=1,2, \ldots N-2, j=1,2, \ldots M-2 \\
U_{0, j}=g(0, j \Delta y) & j=0,1, \ldots M-1 \\
U_{N-1, j}=g(a, j \Delta y) & j=0,1, \ldots M-1 \\
U_{i, 0}=g(i \Delta x, 0) & i=0,1, \ldots N-1 \\
U_{i, M-1}=g(i \Delta x, M-1) & i=0,1, \ldots N-1,
\end{array}
$$

onde $\Delta x=a /(N-1) \Delta y=b /(M-1)$. É possível mostrar que esse esquema é estável e convergente sob certas condições na condição de contorno.

Um exemplo de equação hiperbólica é a equação da onda 


$$
\begin{array}{ll}
u_{t t}-c^{2} u_{x x}=0 & 0<x<\pi, t>0 \\
u(0, t)=u(\pi, t)=0 & t>0 \\
u(x, 0)=f(x) & 0 \leq x \leq \pi \\
u_{t}(x, 0)=g(x) & 0 \leq x \leq \pi .
\end{array}
$$

Um possível esquema numérico para este problema é

$$
\begin{array}{ll}
\frac{U_{i, n+1}-2 U_{i, n}+U_{i, n-1}}{(\Delta y)^{2}}-c^{2} \frac{U_{i+1, n}-2 U_{i, n}+U_{i-1, n}}{(\Delta x)^{2}} & 1 \leq i \leq N-2,0 \leq n \\
U_{0, n}=U_{N-1, n}=0 & 0 \leq n \\
U_{i, 0}=f\left(x_{i}\right) & \\
\frac{U_{i, 1}-U_{i,-1}}{2 \Delta t}=g\left(x_{i}\right) . &
\end{array}
$$

A solução numérica de equações hiperbólicas apresentam uma maior complexidade que a das equação elípticas e parabólicas. As soluções das equações hiperbólicas podem apresentar singularidades.

\subsection{Método das linhas}

A ideia básica do método das linhas é substituir as derivadas da EDP por aproximações algébricas, deixando, sem substituição, as derivadas com relação a uma variável independente. Normalmente a variável escolhida é o tempo. Assim, podemos aproximar a EDP por um sistema de EDOs, e assim usar um método numérico para sistemas de EDOs, e assim calcular uma solução numérica para a EDP. Vamos exemplificar o método com a seguinte equação:

$$
\frac{\partial u}{\partial t}+a \frac{\partial u}{\partial x}=0
$$

com as seguintes condições:

$$
\begin{aligned}
& u(x, 0)=f(x) \\
& u(0, t)=g(t) .
\end{aligned}
$$

Discretizando $\frac{\partial u}{\partial x}$ por uma diferença finita, por exemplo

$$
\frac{\partial u}{\partial x} \approx \frac{u_{i}-u_{i-1}}{\Delta x},
$$

onde $i(1 \leq i \leq M)$ é um índice que designa uma posição ao longo de uma grade em $x$, com $M$ pontos, e $\Delta x$ é o espaçamento em x ao longo da grade. Então a aproximação da equação (3.14) fica

$$
\frac{\partial u_{i}}{\partial t}=-a \frac{u_{i}-u_{i-1}}{\Delta x}, \quad 1 \leq i \leq M .
$$


Observe que (3.18) representa um sistema de $M$ EDOs com apenas uma variável independente t. A condição inicial (3.15) gera $M$ condições iniciais para o sistema (3.18)

$$
u\left(x_{i}, 0\right)=f\left(x_{i}\right), \quad 1 \leq i \leq M .
$$

Da condição (3.16) para $i=1$ temos

$$
u\left(x_{1}, t\right)=g(t)
$$

Assim, as equações (3.18) e as condições (3.15) e (3.16) formam a aproximação completa, pelo método das linhas, da equação (3.14) com as condições (3.15) e (3.16). A solução do sistema de EDOs fornecerá $M$ conjunto de funções $\left\{u_{1}(t), \ldots, u_{M}(t)\right\}$ que é uma aproximação de $(x, t)$ nos pontos da grade correspondentes a $i=1, \ldots, M$. 


\section{Capítulo 4}

\section{Ruídos}

Os estimadores descritos no capítulo 5, assumem que as soluções observadas da EDP (5.1) possuem um ruído. Assim, para a análise destes estimadores precisamos gerar ruídos, com as propriedades esperadas, para obter soluções com ruídos a partir de soluções exatas, ou de soluções numéricas da $\operatorname{EDP}(5.1)$. As soluções são determinadas em $[0, L] \times[0, T]$, os intervalos $[0, L]$ e $[0, T]$ são divididos em $\mathrm{M}$ intervalos, gerando $\mathrm{M}+1$ pontos para cada intervalo, e uma grade de $(M+1)^{2}$ pontos em $[0, L] \times[0, T]$. Assim, o conjunto de $(M+1)^{2}$ pontos representa uma solução $\left(x_{i}, t_{j}, u_{i j}\right), 0 \leq i \leq M, 0 \leq j \leq M, x_{i}=i \frac{L}{M}, t_{j}=i \frac{T}{M}, u_{i j}=u\left(x_{i}, t_{j}\right)$. Supomos que os ruídos tenham média zero, e não sejam correlacionados. Os ruídos serão gerados sobre esse conjunto de pontos. Vamos trabalhar com três tipos de ruídos.

\subsection{Ruído tipo 1}

O Ruído tipo 1, que tem representação no domínio de frequência, é gerado pela expressão 4.1, calculada na grade em $[0, L] \times[0, T]$. Este ruído é baseado no ruído descrito no capítulo 5 seção 5.1 .2 ,

$$
\begin{aligned}
r\left(x_{i}, t_{j}\right)= & \sum_{k=1}^{m} \sum_{l=1}^{n}\left(a_{k l}^{1} \sin \left(b_{k} x_{i}\right) \sin \left(c_{l} t_{j}\right)+a_{k l}^{2} \sin \left(b_{k} x_{i}\right) \cos \left(c_{l} t_{j}\right)+\right. \\
& \left.a_{k l}^{3} \cos \left(b_{k} x_{i}\right) \sin \left(c_{l} t_{j}\right)+a_{k l}^{4} \cos \left(b_{k} x_{i}\right) \cos \left(c_{l} t_{j}\right)\right),
\end{aligned}
$$

onde $\left(x_{i}, t_{j}\right) \in[0, L] \times[0, T], a_{k l}^{1}, a_{k l}^{2}, a_{k l}^{3}$ e $a_{k l}^{4}$ são iid $\frac{A}{(k+l)^{2}} U([-1,1])$, em que $U([-1,1])$ uma variável aleatória com distribuição uniforme no intervalo $[-1,1], b_{k}=2 \pi(F+k)$ e $c_{l}=2 \pi(F+l)$. Ajustando os parâmetros A e F obtemos um ruído como no exemplo 4.1: 


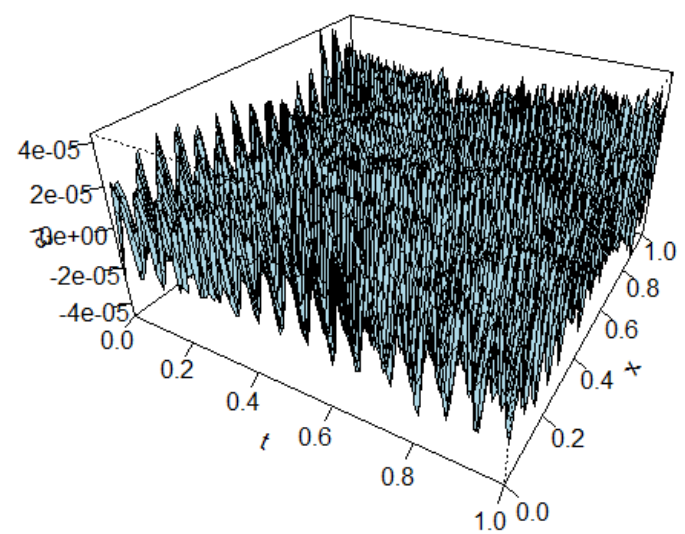

Figura 4.1: Ruído tipo 1

\subsection{Ruído tipo 2}

O Ruído tipo 2 é gerado pela seguinte expressão

$$
r\left(x_{i}, t_{j}\right)=a_{i j},
$$

onde $a_{i j}$ são iid com distribuição $N\left(0, \sigma^{2}\right)$. Claramente esse ruído satisfaz a condição $\mathbb{E}\left(r\left(x_{i}, t_{j}\right)\right)=$ 0 e $\operatorname{Cov}\left(r\left(x_{i}, t_{j}\right), r\left(x_{k}, t_{l}\right)\right)=0, i \neq k$ ou $j \neq l$. Ajustando $\sigma^{2}$ obtemos um como no exemplo 4.2.

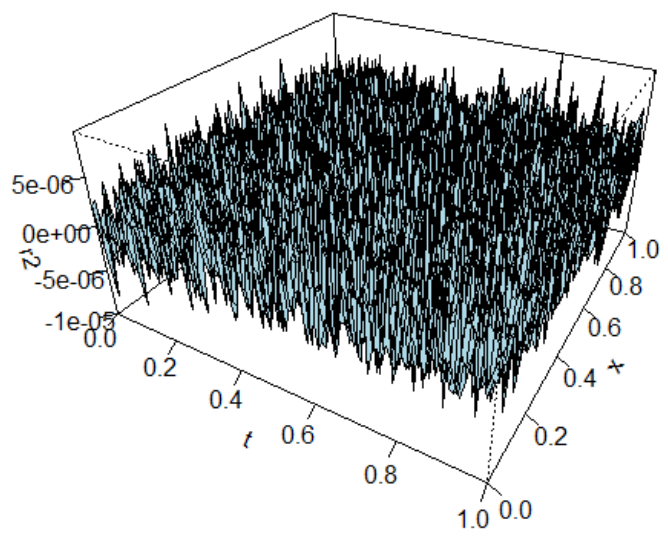

Figura 4.2: Ruído tipo 2 


\subsection{Ruído tipo 3}

O Ruído tipo 3 é baseado no artigo de [WC10]. É um método para simular um processo estacionário gaussiano em uma grade retangular fina em $[0,1]^{d} \subset \mathbb{R}^{d}$. Esse método utiliza métodos de Fourier discretos, a transformada rápida de Fourier e uma nova característica que torna a simulação exata em princípio, ou seja, a simulação teria exatamente uma distribuição multivariada normal se dispuséssemos de um gerador de números realmente aleatórios e independentes, e não tivéssemos imprecisões na aritmética computacional.

Os detalhes do algorítimo deste ruído pode ser visto em [WC10]. Nas nossas simulações utilizamos o pacote RandomFields do R, que fornece uma implementação deste algorítimo. Ajustando os parâmetros, do algorítimo, obtemos um ruído como no exemplo 4.3.

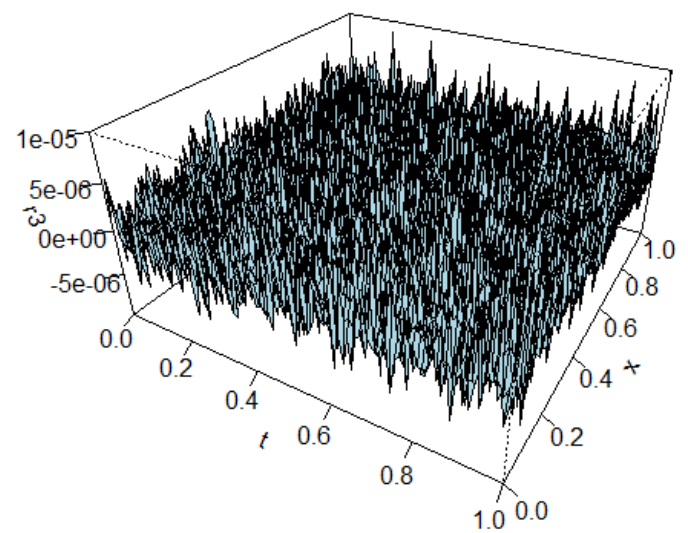

Figura 4.3: Ruído tipo 3 


\section{Capítulo 5}

\section{Estimadores}

Apresentaremos agora os estimadores com integrais, que são objeto de estudo deste trabalho, e os estimadores diretos que serão usados para comparação. Os dois grupos de estimadores estimam os parâmetros funcionais de uma EDP do seguinte tipo:

$$
f \frac{\partial^{2} u}{\partial t^{2}}+g \frac{\partial u}{\partial t}+h u=\frac{\partial}{\partial x}\left(k \frac{\partial u}{\partial x}\right)
$$

onde os parâmetros $f, g, h$ e $k$ são funções que dependem apenas de $x$. Esta EDP tem como casos particulares as equações do calor e da onda com coeficientes funcionais que são utilizados para a modelagem de fenômenos do calor e da onda. Os dois grupos de estimadores assumem a existência de um conjunto de funções com pelo menos quatro funções, que representam soluções da EDP (5.1). As funções são da forma $v=u+\epsilon$ onde $u$ é uma solução da EDP e $\epsilon$ é um ruído não correlacionado. A descrição dos estimadores com integrais foi baseada no trabalho [dM11].

\subsection{Estimadores com integrais}

Nos estimadores com integrais assumimos que temos à disposição $N$ funções $v_{1}(x, t), \cdots, v_{N}(x, t)$ em que $v_{i}=u_{i}+\epsilon_{i}$ para todo $i, 1 \leq i \leq N$ onde $u_{i}$ é uma solução da EDP e $\epsilon_{i}$ é o ruído não correlacionado que satisfaz uma condição no domínio de frequência. As funções $v_{i}$ são bem conhecidas mas não temos informações sobre as soluções $u_{i}$ e nem sobre as realizações dos ruídos aleatórios $\epsilon_{i}$. As variáveis $x$ e $t$ pertencem aos intervalos $[0, L]$ e $[0, T]$ respectivamente. Com base nesta suposições iremos obter estimadores consistentes $\hat{f}, \hat{g}, \hat{h}$ e $\hat{k}$, para $f, g, h$ e $k$.

\subsubsection{Obtendo o estimador}

Para simplificar a apresentação, vamos supor que o número de funções disponíveis seja um múltiplo de quatro, $N=4 n$, e vamos dividir as funções em quatro conjuntos com $n$ elementos, $I_{1}=$ $\left\{v_{1}, \cdots, v_{n}\right\}, I_{2}=\left\{v_{n+1}, \cdots, v_{2 n}\right\}, I_{3}=\left\{v_{2 n+1}, \cdots, v_{3 n}\right\}$ e $I_{4}=\left\{v_{3 n+1}, \cdots, v_{4 n}\right\}$, por exemplo. Sejam $\lambda$ e $\mu$ números reais no intervalo de $(0,1)$.

Integrando parcialmente em relação à segunda variável ao longo do intervalo $[\lambda T-\mu \lambda t, \lambda T+\mu(1-\lambda) t]$ seguido da integração com respeito a $t$ sobre $[0, T]$ e integrando em relação a $(\lambda, \mu) \in(0,1)^{2}$ da equação (5.1) fornece: 


$$
\begin{gathered}
f(x) \int_{0}^{T} u(x, t) Q(t) d t+g(x)\left(\int_{0}^{T} u(x, t) R(t) d t\right)+ \\
h(x) \int_{0}^{T} u(x, t) S(t) d t=\frac{\partial}{\partial x}\left[k(x) \frac{\partial}{\partial x} \int_{0}^{T} u(x, t) S(t) d t\right]
\end{gathered}
$$

onde

$$
\begin{gathered}
Q(t)=\frac{6 t^{2}-6 T t+T^{2}}{6 T^{3}} \\
R(t)=\frac{-2 t^{3}+3 t^{2} T-t T^{2}}{6 T^{3}} \\
S(t)=\frac{(T-t)^{2} t^{2}}{12 T^{3}}
\end{gathered}
$$

Denotando $\bar{u}^{j}=\frac{\sum_{i \in I_{j}} u_{i}}{n}$, devido à linearidade da equação integro-diferencial parcial (EIDP) acima, podemos escrever,

para $1 \leq j \leq 4$,

$$
\begin{gathered}
f(x) \int_{0}^{T} \bar{u}^{j}(x, t) Q(t) d t+g(x)\left(\int_{0}^{T} \bar{u}^{j}(x, t) R(t) d t\right)+ \\
h(x) \int_{0}^{T} \bar{u}^{j}(x, t) S(t) d t=\frac{\partial}{\partial x}\left[k(x) \frac{\partial}{\partial x} \int_{0}^{T} \bar{u}^{j}(x, t) S(t) d t\right]
\end{gathered}
$$

Seja

$$
\begin{aligned}
& \alpha\left(\bar{u}^{j}, x\right)=\alpha_{j}=\int_{0}^{T} \bar{u}^{j}(x, t) Q(t) d t \\
& \beta\left(\bar{u}^{j}, x\right)=\beta_{j}=\int_{0}^{T} \bar{u}^{j}(x, t) R(t) d t
\end{aligned}
$$

$\mathrm{e}$

$$
\gamma\left(\bar{u}^{j}, x\right)=\gamma_{j}=\int_{0}^{T} \bar{u}^{j}(x, t) S(t) d t
$$

Assim, podemos escrever o sistema de quatro equações

$$
\alpha_{j}(x) f(x)+\beta_{j}(x) g(x)+\gamma_{j}(x) h(x)=\frac{\partial}{\partial x}\left[k(x) \frac{\partial}{\partial x} \gamma_{j}\right]=k^{\prime}(x) \gamma_{j}^{\prime}+k(x) \gamma_{j}^{\prime \prime}
$$

Denotando

$$
\begin{aligned}
& \alpha=\left(\alpha_{1}, \alpha_{2}, \alpha_{3}, \alpha_{4}\right), \\
& \beta=\left(\beta 1, \beta_{2}, \beta_{3}, \beta_{4}\right),
\end{aligned}
$$




$$
\begin{gathered}
\gamma=\left(\gamma_{1}, \gamma_{2}, \gamma_{3}, \gamma_{4}\right), \\
\left(\gamma_{1}^{\prime}, \gamma_{2}^{\prime}, \gamma_{3}^{\prime}, \gamma_{4}^{\prime}\right) \text { e } \\
\gamma^{\prime \prime}=\left(\gamma_{1}^{\prime \prime}, \gamma_{2}^{\prime \prime}, \gamma_{3}^{\prime \prime}, \gamma_{4}^{\prime \prime}\right),
\end{gathered}
$$

onde $\gamma_{i}^{\prime}=f r a c d \gamma_{i} d x$ e $\gamma_{i}^{\prime \prime}=f r a c d^{2} \gamma_{i} d x^{2}, i=1,2,3,4$

Resolvendo o sistema obtemos

$$
\begin{gathered}
k(z)=k(0) \exp \left(-\int_{0}^{z} \frac{\operatorname{det}\left(\alpha, \beta, \gamma, \gamma^{\prime \prime}\right)}{\operatorname{det}\left(\alpha, \beta, \gamma, \gamma^{\prime}\right)} d x\right) \\
f(x)=k(x) \frac{\operatorname{det}\left(\gamma^{\prime \prime}, \beta, \gamma, \gamma^{\prime}\right)}{\operatorname{det}\left(\alpha, \beta, \gamma, \gamma^{\prime}\right)} \\
g(x)=k(x) \frac{\operatorname{det}\left(\alpha, \gamma^{\prime \prime}, \gamma, \gamma^{\prime}\right)}{\operatorname{det}\left(\alpha, \beta, \gamma, \gamma^{\prime}\right)} \\
h(x)=k(x) \frac{\operatorname{det}\left(\alpha, \beta, \gamma^{\prime \prime}, \gamma^{\prime}\right)}{\operatorname{det}\left(\alpha, \beta, \gamma, \gamma^{\prime}\right)}
\end{gathered}
$$

Mais brevemente:

$$
\begin{aligned}
& k=K(\alpha, \beta, \gamma)=K^{*}\left(\bar{u}^{1}, \bar{u}^{2}, \bar{u}^{3}, \bar{u}^{4}\right) \\
& f=F(\alpha, \beta, \gamma)=F^{*}\left(\bar{u}^{1}, \bar{u}^{2}, \bar{u}^{3}, \bar{u}^{4}\right) \\
& g=G(\alpha, \beta, \gamma)=G^{*}\left(\bar{u}^{1}, \bar{u}^{2}, \bar{u}^{3}, \bar{u}^{4}\right) \\
& h=H(\alpha, \beta, \gamma)=H^{*}\left(\bar{u}^{1}, \bar{u}^{2}, \bar{u}^{3}, \bar{u}^{4}\right)
\end{aligned}
$$

Denotando $\bar{v}^{i}=\frac{\sum_{i \in I_{j}} v_{i}}{n}$, para $1 \leq j \leq 4$, definindo $\hat{\alpha}_{j}, \hat{\beta}_{j}$ e $\hat{\gamma}_{j}$ pela substituição de $\bar{u}^{i}$ por $\bar{v}^{i}$ nas equações (5.4), (5.5) e (5.6), e utilizando as notações de vetores.

Agora, definimos nossos estimadores para os coeficientes funcionais como

$$
\begin{aligned}
& k=K(\hat{\alpha}, \hat{\beta}, \hat{\gamma})=K^{*}\left(\bar{v}^{1}, \bar{v}^{2}, \bar{v}^{3}, \bar{v}^{4}\right) \\
& f=F(\hat{\alpha}, \hat{\beta}, \hat{\gamma})=F^{*}\left(\bar{v}^{1}, \bar{v}^{2}, \bar{v}^{3}, \bar{v}^{4}\right) \\
& g=G(\hat{\alpha}, \hat{\beta}, \hat{\gamma})=G^{*}\left(\bar{v}^{1}, \bar{v}^{2}, \bar{v}^{3}, \bar{v}^{4}\right) \\
& h=H(\hat{\alpha}, \hat{\beta}, \hat{\gamma})=H^{*}\left(\bar{v}^{1}, \bar{v}^{2}, \bar{v}^{3}, \bar{v}^{4}\right)
\end{aligned}
$$


Observamos que o modelo (5.1) é não identificável como pode ser visto claramente pela presença da constante $k(0)$ em (5.8), e, consequentemente, em (5.9), (5.10) e (5.11). No entanto, se, por exemplo, sabemos que $\int_{0}^{L} k(x) d x=y$ então, a partir de (5.7), temos que,

$$
k(0)=y\left[\int_{0}^{L} \exp \left(\frac{\operatorname{det}\left(\alpha, \beta, \gamma, \gamma^{\prime \prime}\right)}{\operatorname{det}\left(\alpha, \beta, \gamma, \gamma^{\prime}\right)} d x\right)\right]^{-1}
$$

e a identificabilidade é garantida.

\subsubsection{Resultados principais}

Vamos supor que o ruído tem uma representação no domínio de frequência. Usando a notação complexa escrevemos

$$
\epsilon_{i}(x, t)=\sum_{l, m \in \mathbb{Z}} a_{i, l, m} e^{l\left(\frac{2 \pi l x}{L}+\frac{2 \pi m t}{T}\right)}
$$

De agora em diante, vamos supor que o ruído satisfaz as seguintes condições:

1. para todo $i, l$ e $m, \mathbb{E} a_{i, l, m}=0$;

2. para todo $i, j, l, m, p$ e $q$, tais que $(i, l, m) \neq(j, p, q)$, $\operatorname{Cov}\left(a_{i, l, m}, a_{j, p, q}\right)=0$;

3. $\frac{1}{n^{2}} \sum_{i=1}^{n} \sum_{l, m \in \mathbb{Z}} l^{4} \mathbb{E}\left(a_{i, l, m}^{2}\right) \rightarrow 0$ quando $n \rightarrow \infty$

Claramente, a condição [3] é cumprida no caso em que ruído é identicamente distribuído (o que significa que, no domínio de frequência, para todo $i$ e $j$ e para todo $l$ e $m$, temos $a_{i, l, m}=a_{j, p, q}$ ) e satisfaz $\sum_{l, m \in \mathbb{Z}} l^{4} \mathbb{E}\left(a_{i, l, m}^{2}\right)$. A condição [2] impõe o não correlacionamento dos coeficientes inter e intra do ruído. No caso, temos os ruídos independentes aos pares, ou seja, $\epsilon_{i} \perp \epsilon_{j}$, para $i \neq j$, ainda precisamos do cumprimento de $\mathbb{C o v}\left(a_{i, l, m}, a_{j, p, q}\right)=0$, a fim de obedecer [2]. Este é também o caso se tivermos ruídos independentes.

Observe que o Teorema 1, Corolário 1 e Teorema 2 dizem respeito a forma de estimação. Podemos assumir um valor arbitrário diferente de zero para $\hat{k}(0)=k(0)$ ou considerar os representantes padrões da classe, para emparelhar as funções. Usando a notação estabelecida até agora, podemos afirmar o seguinte:

Teorema 1. Sejam os coeficientes funcionais $f, g$, $h$ e $k$ que aparecem em (5.1) tais que $(f, g, h, k) \neq$ $(0,0,0,0)$ e, para todo $x \in[0, L]$, $\operatorname{det}\left(\alpha, \beta, \gamma, \gamma^{\prime}\right) \neq 0$. Supondo que os ruído satisfazem as condições 1,2 e 3 acima. Então, a média integrada dos erros quadráticos $\mathbb{E}\left(\|\hat{k}-k\|_{2}^{2}\right), \mathbb{E}\left(\|\hat{f}-f\|_{2}^{2}\right)$, $\mathbb{E}\left(\|\hat{g}-g\|_{2}^{2}\right)$ e $\mathbb{E}\left(\|\hat{h}-h\|_{2}^{2}\right)$ tendem a zero quando $N \longrightarrow \infty$.

Este resultado é independente do valor de $k(0)$. A condição $\operatorname{det}\left(\alpha, \beta, \gamma, \gamma^{\prime}\right) \neq 0$ implica que $\left\{\hat{u}_{1}, \hat{u}_{2}, \hat{u}_{3}, \hat{u}_{4}\right\}$ são linearmente independentes. 
Corolário 1. Sob as mesmas hipóteses do Teorema 1, os estimadores $\hat{f}, \hat{g}, \hat{h}$ e $\hat{k}$ são consistentes,

Teorema 2. Sob as hipóteses do Teorema 1 e no pressuposto de que o ruído é i.i.d. como $\epsilon$, nós te$\operatorname{mos} \sqrt{N}(\hat{f}-f) \rightsquigarrow T_{f}(v), \sqrt{N}(\hat{g}-g) \rightsquigarrow T_{g}(v), \sqrt{N}(\hat{h}-h) \rightsquigarrow T_{h}(v) e \sqrt{N}(\hat{k}-k) \rightsquigarrow T_{k}(v)$, onde

$T_{f}(v)=F^{*^{\prime}}\left(\hat{u}^{1}, \hat{u}^{2}, \hat{u}^{3}, \hat{u}^{4}\left(v_{1}, v_{2}, v_{3}, v_{4}\right)\right)$,

$T_{g}(v)=G^{*^{\prime}}\left(\hat{u}^{1}, \hat{u}^{2}, \hat{u}^{3}, \hat{u}^{4}\left(v_{1}, v_{2}, v_{3}, v_{4}\right)\right)$,

$T_{h}(v)=H^{*^{\prime}}\left(\hat{u}^{1}, \hat{u}^{2}, \hat{u}^{3}, \hat{u}^{4}\left(v_{1}, v_{2}, v_{3}, v_{4}\right)\right)$,

$e T_{j}(v)=K^{*^{\prime}}\left(\hat{u}^{1}, \hat{u}^{2}, \hat{u}^{3}, \hat{u}^{4}\left(v_{1}, v_{2}, v_{3}, v_{4}\right)\right)$.

Aqui $\left\{v_{1}, v_{2}, v_{3}, v_{4}\right\}$ são i.i.d. com distribuição idêntica à de $v$ e $v$ tem representação espectral dada por

$v(x, t)=\sum_{l, m \in \mathbb{Z}} b_{l, m} e^{l\left(\frac{2 \pi l x}{L}+\frac{2 \pi m t}{T}\right)}$ com coeficientes não correlacionadas $b_{l, m} \sim N\left(0, \sigma_{l, m}^{2}\right)$, onde $\sigma_{l, m}^{2}$ é a variância comum de $a_{i, l, m}$.

\subsubsection{Observações}

Observa-se que a metodologia apresentada aqui, ou seja, a integração da EDP numa região convenientemente escolhida, a solução analítica do novo EIDP integrado para os coeficientes funcionais e, finalmente, a substituição das verdadeiras soluções de EDP por médias das soluções medidas nas expressões analíticas para os coeficientes funcionais, pode ser aplicada para uma classe maior de EDPs que incluem EDPs do tipo: $\sum_{i=0}^{m} f_{i} \frac{\partial}{\partial x}\left[k \frac{\partial u}{\partial x}\right]$ e outros tipos.

Podemos definir outros estimadores para $f, g, h$ e $k$ alisando primeiro nossos dados funcionais. Isto pode ser feito, por exemplo, simplesmente por alisamento de $v_{i}$, utilizando um filtro passa-baixo e, em seguida, utilizando as versões suavizados de $v_{i}$ em equações dos estimadores. Esperamos que este procedimento forneça bons resultados em caso de ruído de alta frequência.

As condições que assumimos em relação ao comportamento no domínio da frequência do ruído pode ser relaxada, mais especificamente, pode haver alguma correlação moderada entre os coeficientes de Fourier, tanto ruído inter e intra e da consistência dos estimadores ainda será mantida.

Observe que, embora o estimadores $\hat{f}, \hat{g}, \hat{h}, \hat{k}$, sejam consistentes, eles são, em princípio, dependentes da escolha dos conjuntos $I_{1}, I_{2}, I_{3}, I_{4}$.

\subsection{Estimadores diretos}

Os estimadores diretos utilizam, também, quatro funções $v_{1}, v_{2}, v_{3}$ e $v_{4}$ associadas a EDP (5.1) em que $v_{i}=u_{i}+\epsilon_{i}$.

Definindo:

$$
U(x, t)=\left(u_{1}(x, t), u_{2}(x, t), u_{3}(x, t), u_{4}(x, t)\right)
$$


Sejam

$$
\begin{gathered}
\frac{\partial^{2} U}{\partial t^{2}}(x, t)=\left(\frac{\partial^{2} u_{1}}{\partial t^{2}}(x, t), \frac{\partial^{2} u_{2}}{\partial t^{2}}(x, t), \frac{\partial^{2} u_{3}}{\partial t^{2}}(x, t), \frac{\partial^{2} u_{4}}{\partial t^{2}}(x, t)\right), \\
\frac{\partial U}{\partial t}(x, t)=\left(\frac{\partial u_{1}}{\partial t}(x, t), \frac{\partial u_{2}}{\partial t}(x, t), \frac{\partial u_{3}}{\partial t}(x, t), \frac{\partial u_{4}}{\partial t}(x, t)\right), \\
\frac{\partial^{2} U}{\partial x^{2}}(x, t)=\left(\frac{\partial^{2} u_{1}}{\partial x^{2}}(x, t), \frac{\partial^{2} u_{2}}{\partial x^{2}}(x, t), \frac{\partial^{2} u_{3}}{\partial x^{2}}(x, t), \frac{\partial^{2} u_{4}}{\partial x^{2}}(x, t)\right), \\
\frac{\partial U}{\partial x}(x, t)=\left(\frac{\partial u_{1}}{\partial x}(x, t), \frac{\partial u_{2}}{\partial x}(x, t), \frac{\partial u_{3}}{\partial x}(x, t), \frac{\partial u_{4}}{\partial x}(x, t)\right) .
\end{gathered}
$$

Os parâmetros $f, g, h$, e $k$ podem ser obtidos pelas igualdades:

$$
\begin{gathered}
k(z)=k(0) \exp \left(-\int_{0}^{z} \frac{\operatorname{det}\left(\frac{\partial^{2} U}{\partial t^{2}}, \frac{\partial U}{\partial t}, U, \frac{\partial^{2} U}{\partial x^{2}}\right)}{\operatorname{det}\left(\frac{\partial^{2} U}{\partial t^{2}}, \frac{\partial U}{\partial t}, U, \frac{\partial U}{\partial x}\right)} d x\right), \\
f(x)=k(x) \frac{\operatorname{det}\left(\frac{\partial^{2} U}{\partial x^{2}}, \frac{\partial U}{\partial t}, U, \frac{\partial U}{\partial x}\right)}{\operatorname{det}\left(\frac{\partial^{2} U}{\partial t^{2}}, \frac{\partial U}{\partial t}, U, \frac{\partial U}{\partial x}\right)}, \\
g(x)=k(x) \frac{\operatorname{det}\left(\frac{\partial^{2} U}{\partial t^{2}}, \frac{\partial^{2} U}{\partial x^{2}}, U, \frac{\partial U}{\partial x}\right)}{\operatorname{det}\left(\frac{\partial^{2} U}{\partial t^{2}}, \frac{\partial U}{\partial t}, U, \frac{\partial U}{\partial x}\right)}, \\
h(x)=k(x) \frac{\operatorname{det}\left(\frac{\partial^{2} U}{\partial t^{2}}, \frac{\partial U}{\partial t}, \frac{\partial^{2} U}{\partial x^{2}}, \frac{\partial U}{\partial x}\right)}{\operatorname{det}\left(\frac{\partial^{2} U}{\partial t^{2}}, \frac{\partial U}{\partial t}, U, \frac{\partial U}{\partial x}\right)},
\end{gathered}
$$

e os estimadores diretos $\tilde{f}, \tilde{g}, \tilde{h}$ e $\tilde{k}$ são definidos pela substituição de u por v nas equações (5.12) $(5.15)$

Devemos observar que os parâmetros $f, g, h$ e $k$ dependem apenas de $x$. Assim, eles são estimados para um valor arbitrário de $t \in[0, T]$. Teoricamente as estimativas dos parâmetros são independentes do valor de $t$ escolhido. 


\section{Capítulo 6}

\section{Análise e comparação dos estimadores}

Vamos descrever o procedimento utilizado para a comparação e análise dos estimadores com integrais e diretos.

\subsection{Equações selecionadas}

Selecionamos algumas equações, que são casos particulares da equação (5.1), para aplicar os estimadores e analisar os resultados. Selecionamos 11 equações em que é possível encontrar soluções analíticas e uma equação em que a solução será encontrada de forma numérica. As soluções analíticas apresentadas foram retiradas de [Pol02].

\subsubsection{Soluções analíticas}

As soluções analíticas selecionadas são soluções particulares, não estando associadas a condições iniciais e/ou de contorno especificas.

\section{Equações parabólicas}

Equação 1 Fazendo $f(x)=0, g(x)=1, h(x)=0, k(x)=a$, onde $a \in \mathbb{R}$ é uma contante, obtemos a seguinte equação:

$$
\frac{\partial u}{\partial t}=a \frac{\partial^{2} u}{\partial x^{2}}
$$

Temos as seguintes soluções particulares

1. $u=A x^{2}+2 a A t$;

2. $u=A e^{\left(k x+k^{2} a t\right)}+B$;

3. $u=A \cos (k x+C) e^{-k^{2} a t}$;

4. $u=A e^{-\mu x} \cos \left(\mu x-2 a \mu^{2} t+C\right)$, 
onde $A, B, C, k, \mu \in \mathbb{R}$ são constantes.

Equação 2 Fazendo $f(x)=0, g(x)=1, h(x)=-(b x+k), k(x)=a$, onde $a, b, k \in \mathbb{R}$ são constantes, obtemos a seguinte equação:

$$
\frac{\partial u}{\partial t}=a \frac{\partial^{2} u}{\partial x^{2}}+(b x+k) u
$$

Para encontrar soluções aplicamos as seguintes substituições.

$$
\begin{aligned}
& u(x, t)=w(\xi, \tau) e^{t(k+b x)+\frac{1}{3} a b^{2} t^{3}} \\
& \xi=x+a b t^{2} \\
& \tau=t
\end{aligned}
$$

Desta forma a equação se reduz a $\frac{\partial w}{\partial \tau}=a \frac{\partial^{2} w}{\partial \xi^{2}}$ e podemos usar as soluções da equação (6.1) para gerar soluções para a equação original.

1. $e^{\frac{1}{3} a b^{2} t^{3}+t(k+b x)}\left(2 a A t+A\left(a b t^{2}+x\right)^{2}\right)$;

2. $e^{\frac{1}{3} a b^{2} t^{3}+t(k+b x)}\left(A e^{a k_{1}^{2} t+k_{1}\left(a b t^{2}+x\right)}+B\right)$;

3. $A e^{-a k_{1}^{2} t+\frac{1}{3} a b^{2} t^{3}+t(k+b x)} \cos \left(k_{1}\left(a b t^{2}+x\right)+C\right)$;

4. $A e^{\frac{1}{3} a b^{2} t^{3}+t(k+b x)-\left(a b t^{2}+x\right) \mu} \cos \left(\left(a b t^{2}+x\right) \mu-2 a t \mu^{2}+C\right)$,

onde $A, B, C, k_{1}, \mu \in \mathbb{R}$ são constantes.

\section{Equações hiperbólicas}

Equação 3 Fazendo $f(x)=1, g(x)=0, h(x)=0, k(x)=a^{2}$, onde $a \in \mathbb{R}$ é uma constante, obtemos a seguinte equação:

$$
\frac{\partial^{2} u}{\partial t^{2}}=a^{2} \frac{\partial^{2} u}{\partial x^{2}}
$$

Temos as seguintes soluções particulares

1. $u=\cos (x+a t)+\sin (x-a t)$;

2. $u=e^{-(x+a t)^{2}}+\sin \left(-(x-a t)^{2}\right)$;

3. $u=(x+a t)^{2}+e^{\left((x-a t)^{2}\right)}$;

4. $u=(x+a t)^{2}+(x-a t)^{3}$, 
Equação 4 Fazendo $f(x)=1, g(x)=0, h(x)=b, k(x)=a^{2}$, onde $a, b \in \mathbb{R}$ e $b>0$ são constantes, obtemos a seguinte equação:

$$
\frac{\partial^{2} u}{\partial t^{2}}=a^{2} \frac{\partial^{2} u}{\partial x^{2}}-b u
$$

Temos as seguintes soluções particulares

1. $u=e^{\frac{\sqrt{b} x}{a}}(A t+B)$;

2. $u=e^{-\frac{\sqrt{b} x}{a}}(A t+B)$;

3. $u=\cos (\lambda x)\left(A \cos \left(\sqrt{\lambda^{2} a^{2}+b} t\right)+B \sin \left(\sqrt{\lambda^{2} a^{2}+b} t\right)\right)$;

4. $u=\sin (\lambda x)\left(A \cos \left(\sqrt{\lambda^{2} a^{2}+b} t\right)+B \sin \left(\sqrt{\lambda^{2} a^{2}+b} t\right)\right)$;

5. $u=\exp (\lambda x)\left(A \cos \left(\sqrt{b-\lambda^{2} a^{2}} t\right)+B \sin \left(\sqrt{b-\lambda^{2} a^{2}} t\right)\right)$;

6. $u=\exp (\lambda x)\left(A \exp \left(\sqrt{b-\lambda^{2} a^{2}} t\right)+B \exp \left(-\sqrt{b-\lambda^{2} a^{2}} t\right)\right)$,

onde $A, B, \lambda \in \mathbb{R}$ são constantes.

Equação 5 Fazendo $f(x)=1, g(x)=k, h(x)=b, k(x)=a^{2}$, onde $a, b, k \in \mathbb{R}$ e $b>0$ são constantes, obtemos a seguinte equação:

$$
\frac{\partial^{2} u}{\partial t^{2}}+k \frac{\partial u}{\partial t}=a^{2} \frac{\partial^{2} u}{\partial x^{2}}-b u
$$

Para encontrar soluções aplicamos a seguinte substituição.

$$
u(x, t)=e^{-\frac{1}{2} k t} w(x, t)
$$

Desta forma a equação se reduz a $\frac{\partial^{2} w}{\partial t^{2}}=a^{2} \frac{\partial^{2} w}{\partial x^{2}}-\left(b-\frac{1}{4} k^{2}\right) w$ e podemos usar as soluções da equação (6.4) para gerar para gerar soluções para a equação original

1. $u=e^{-\frac{k t}{2}+\frac{\sqrt{b-\frac{k^{2}}{4} x}}{a}}(A t+B)$;

2. $u=e^{-\frac{k t}{2}-\frac{\sqrt{b-\frac{k^{2}}{4} x}}{a}}(A t+B)$;

3. $u=e^{-\frac{k t}{2}} \cos (\lambda x)\left(A \cos \left(t \sqrt{b-\frac{k^{2}}{4}+\lambda^{2} a^{2}}\right)+B \sin \left(t \sqrt{b-\frac{k^{2}}{4}+\lambda^{2} a^{2}}\right)\right)$; 
4. $u=e^{-\frac{k t}{2}} \sin (\lambda x)\left(A \cos \left(t \sqrt{b-\frac{k^{2}}{4}+\lambda^{2} a^{2}}\right)+B \sin \left(t \sqrt{b-\frac{k^{2}}{4}+\lambda^{2} a^{2}}\right)\right)$

5. $u=e^{-\frac{k t}{2}+\lambda x}\left(A \cos \left(\sqrt{b-\frac{k^{2}}{4}-\lambda^{2} a^{2} t}\right)+B \sin \left(\sqrt{b-\frac{k^{2}}{4}-\lambda^{2} a^{2}} t\right)\right) ;$

6. $u=e^{-\frac{k t}{2}+\lambda x}\left(A \exp \left(\sqrt{-b+\frac{k^{2}}{4}+\lambda^{2} a^{2} t}\right)+B \exp \left(-\sqrt{-b+\frac{k^{2}}{4}+\lambda^{2} a^{2} t}\right)\right)$,

onde $A, B, \lambda \in \mathbb{R}$ são constantes.

\section{Equações elípticas}

Equação 6 Fazendo $f(x)=1, g(x)=0, h(x)=0, k(x)=-1$ obtemos a seguinte equação:

$$
\frac{\partial^{2} u}{\partial t^{2}}+\frac{\partial^{2} u}{\partial x^{2}}=0
$$

Temos as seguintes soluções particulares.

1. $u=A t+B x+C$;

2. $u=A\left(t^{2}-x^{2}\right)+B t x$;

3. $u=A\left(t^{3}-3 t x^{2}\right)+B\left(3 t^{2} x-x^{3}\right)$;

4. $u=\frac{A t+B x}{t^{2}+x^{2}}+C$

5. $u=e^{-\mu t}(A \cos (\mu x)+B \sin (\mu x))$;

6. $u=e^{-\mu x}(A \cos (\mu t)+B \sin (\mu t))$;

7. $u=(A \cosh (\mu t)+B \sinh (\mu t))(C \cos (\mu x)+D \sin (\mu x))$;

8. $u=(A \cosh (\mu x)+B \sinh (\mu x))(C \cos (\mu t)+D \sin (\mu t))$;

9. $u=A \ln \left(\left(t-t_{0}\right)^{2}+\left(x-x_{0}\right)^{2}\right)+B$,

onde $A, B, C, \mu, x_{0}, t_{0} \in \mathbb{R}$ são constantes. 
Equação 7 Fazendo $f(x)=1, g(x)=0, h(x)=\lambda, k(x)=-1$, onde $\lambda \in \mathbb{R}$ e $\lambda>0$ é uma constante, obtemos a seguinte equação:

$$
\frac{\partial^{2} u}{\partial t^{2}}+\frac{\partial^{2} u}{\partial x^{2}}+\lambda u=0
$$

Temos as seguintes soluções particulares

1. $u=(A t+B)(C \cos (\sqrt{\lambda} x)+D \sin (\sqrt{\lambda} x))$

2. $u=(A x+B)(C \cos (\sqrt{\lambda} t)+D \sin (\sqrt{\lambda} t))$

3. $u=\left(A \cos \left(\sqrt{\frac{\lambda}{2}} t\right)+B \sin \left(\sqrt{\frac{\lambda}{2}} t\right)\right)\left(C \cos \left(\sqrt{\frac{\lambda}{2}} x\right)+D \sin \left(\sqrt{\frac{\lambda}{2}} x\right)\right)$

4. $u=\left(A \cos \left(2 \sqrt{\frac{\lambda}{3}} t\right)+B \sin \left(2 \sqrt{\frac{\lambda}{3}} t\right)\right)\left(C \cosh \left(\sqrt{\frac{\lambda}{3}} x\right)+D \sinh \left(\sqrt{\frac{\lambda}{3}} x\right)\right)$

5. $u=\left(A \cosh \left(\sqrt{\frac{\lambda}{3}} t\right)+B \sinh \left(\sqrt{\frac{\lambda}{3}} t\right)\right)\left(C \cos \left(2 \sqrt{\frac{\lambda}{3}} x\right)+D \sin \left(2 \sqrt{\frac{\lambda}{3}} x\right)\right)$,

onde $A, B, C, D, \lambda \in \mathbb{R}$ são constantes.

Equação 8 Fazendo $f(x)=-1, g(x)=0, h(x)=a e^{\beta x}, k(x)=-1$, onde $a, \beta \in \mathbb{R}$ são constantes, obtemos a seguinte equação:

$$
\frac{\partial^{2} u}{\partial t^{2}}+\frac{\partial^{2} u}{\partial x^{2}}=a e^{\beta x} u
$$

Para encontrar soluções aplicamos as seguintes substituições.

$$
\begin{aligned}
& v(x, t)=e^{\frac{1}{2} \beta x} \cos \left(\frac{1}{2} \beta t\right) \\
& w(x, t)=e^{\frac{1}{2} \beta x} \sin \left(\frac{1}{2} \beta t\right) .
\end{aligned}
$$

Desta forma a equação se reduz a $\frac{\partial^{2} u}{\partial v^{2}}+\frac{\partial^{2} u}{\partial w^{2}}+4 a \beta^{-2} u=0$ e podemos usar as soluções da equação (6.6) para gerar soluções para a equação original

1. $u=\left(A e^{\frac{\beta x}{2}} \cos \left(\frac{\beta t}{2}\right)+B\right)\left(C \cosh \left(2 e^{\frac{\beta x}{2}} \sqrt{\frac{a}{\beta^{2}}} \sin \left(\frac{\beta t}{2}\right)\right)+D \sinh \left(2 e^{\frac{\beta x}{2}} \sqrt{\frac{a}{\beta^{2}}} \sin \left(\frac{\beta t}{2}\right)\right)\right) ;$

2. $u=\left(A e^{\frac{\beta x}{2}} \sin \left(\frac{\beta t}{2}\right)+B\right)\left(C \cosh \left(2 e^{\frac{\beta x}{2}} \sqrt{\frac{a}{\beta^{2}}} \cos \left(\frac{\beta t}{2}\right)\right)+D \sinh \left(2 e^{\frac{\beta x}{2}} \sqrt{\frac{a}{\beta^{2}}} \cos \left(\frac{\beta t}{2}\right)\right)\right)$

3. $u=\left(A \cos \left(2 e^{\frac{\beta x}{2}} \sqrt{\frac{a}{3 \beta^{2}}} \cos \left(\frac{\beta t}{2}\right)\right)+B \sin \left(2 e^{\frac{\beta x}{2}} \sqrt{\frac{a}{3 \beta^{2}}} \cos \left(\frac{\beta t}{2}\right)\right)\right)$;

$$
\left(C \cosh \left(4 e^{\frac{\beta x}{2}} \sqrt{\frac{a}{3 \beta^{2}}} \sin \left(\frac{\beta t}{2}\right)\right)+D \sinh \left(4 e^{\frac{3 \beta x}{2}} \sqrt{\frac{a}{3 \beta^{2}}} \sin \left(\frac{\beta t}{2}\right)\right]\right) \text {; }
$$


4. $u=\left(A \cosh \left(4 e^{\frac{\beta x}{2}} \sqrt{\frac{a}{3 \beta^{2}}} \cos \left(\frac{\beta t}{2}\right)\right)+B \sinh \left(4 e^{\frac{\beta x}{2}} \sqrt{\frac{a}{3 \beta^{2}}} \cos \left(\frac{\beta t}{2}\right)\right)\right)$;

$\left(C \cos \left(2 e^{\frac{\beta x}{2}} \sqrt{\frac{a}{3 \beta^{2}}} \sin \left(\frac{\beta t}{2}\right)\right)+D \sin \left(2 e^{\frac{\beta x}{2}} \sqrt{\frac{a}{3 \beta^{2}}} \sin \left(\frac{\beta t}{2}\right)\right)\right)$;

5. $u=\left(A \cosh \left(e^{\frac{\beta x}{2}} \sqrt{\frac{2 a}{\beta^{2}}} \cos \left(\frac{\beta t}{2}\right)\right)+B \sinh \left(e^{\frac{\beta x}{2}} \sqrt{\frac{2 a}{\beta^{2}}} \cos \left(\frac{\beta t}{2}\right)\right)\right)$;

$\left(C \cosh \left(e^{\frac{\beta x}{2}} \sqrt{\frac{2 a}{\beta^{2}}} \sin \left(\frac{\beta t}{2}\right)\right)+D \sinh \left(e^{\frac{\beta x}{2}} \sqrt{\frac{2 a}{\beta^{2}}} \sin \left(\frac{\beta t}{2}\right)\right)\right)$,

onde $A, B, C, D \in \mathbb{R}$ são constantes.

Equação 9 Fazendo $f(x)=1, g(x)=-\alpha, h(x)=0, k(x)=-1$, onde $\alpha \in \mathbb{R}$ é uma constante, obtemos a seguinte equação:

$$
\frac{\partial^{2} u}{\partial t^{2}}-\alpha \frac{\partial u}{\partial t}+\frac{\partial^{2} u}{\partial x^{2}}=0
$$

Para encontrar soluções aplicamos as seguintes substituições

$$
u(x, t)=e^{\frac{1}{2} \alpha t} w(x, t) .
$$

Desta forma a equação se reduz a $\frac{\partial^{2} w}{\partial t^{2}}+\frac{\partial^{2} w}{\partial x^{2}}=\frac{1}{4} \alpha^{2} w$ e podemos usar as soluções da equação (6.7) para gerar soluções para a equação original

1. $u=e^{\frac{\alpha t}{2}}(A t+B)\left(C \cosh \left(\frac{\alpha x}{2}\right)+D \sinh \left(\frac{\alpha x}{2}\right)\right)$;

2. $u=e^{\frac{\alpha t}{2}}(A x+B)\left(C \cosh \left(\frac{\alpha t}{2}\right)+D \sinh \left(\frac{\alpha t}{2}\right)\right)$;

3. $u=e^{\frac{\alpha t}{2}}\left(A \cos \left(\frac{\sqrt{\alpha^{2}} t}{2 \sqrt{3}}\right)+B \sin \left(\frac{\sqrt{\alpha^{2}} t}{2 \sqrt{3}}\right)\right)\left(C \cosh \left(\frac{\sqrt{\alpha^{2}} x}{\sqrt{3}}\right)+D \sinh \left(\frac{\sqrt{\alpha^{2}} x}{\sqrt{3}}\right)\right)$;

4. $u=e^{\frac{\alpha t}{2}}\left(C \cos \left(\frac{\sqrt{\alpha^{2}} x}{2 \sqrt{3}}\right)+D \sin \left(\frac{\sqrt{\alpha^{2}} x}{2 \sqrt{3}}\right)\right)\left(A \cosh \left(\frac{\sqrt{\alpha^{2}} t}{\sqrt{3}}\right]+B \sinh \left(\frac{\sqrt{\alpha^{2}} t}{\sqrt{3}}\right)\right)$;

5. $u=e^{\frac{\alpha t}{2}}\left(A \cosh \left(\frac{\sqrt{\alpha^{2}} t}{2 \sqrt{2}}\right)+B \sinh \left(\frac{\sqrt{\alpha^{2}} t}{2 \sqrt{2}}\right)\right)\left(C \cosh \left(\frac{\sqrt{\alpha^{2}} x}{2 \sqrt{2}}\right)+D \sinh \left(\frac{\sqrt{\alpha^{2}} x}{2 \sqrt{2}}\right)\right)$,

onde $A, B, C, D \in \mathbb{R}$ são constantes.

Equação 10 Fazendo $f(x)=1, g(x)=0, h(x)=0, k(x)=-p(x)$, onde $p(x)$ é uma função de classe $C^{1}$, obtemos a seguinte equação:

$$
\frac{\partial^{2} u}{\partial t^{2}}=\frac{\partial}{\partial x}\left(-p(x) \frac{\partial u}{\partial x}\right)
$$


Temos as seguintes soluções particulares

- $u=C_{1} t^{2}+C_{2} t-2 \int \frac{C_{1} x+C_{3}}{p(x)} d x+C_{4}$;

- $u=C_{1} t^{3}+C_{2} t-6 t \int \frac{C_{1} x+C_{3}}{p(x)} d x+C_{4}$;

- $u=\left(C_{1} \Phi(x)+C_{2}\right) t+C_{3} \Phi(x)+C_{4}, \Phi(x)=\int \frac{d x}{p(x)}$;

- $u=\left(C_{1} \Phi(x)+C_{2}\right) t^{2}+C_{3} \Phi(x)+C_{4}-2 \int\left(\frac{1}{p(x)} \int\left(C_{1} \Phi(x)+C_{2}\right) d x\right)$,

onde $C_{1}, C_{2}, C_{3}, C_{4} \in \mathbb{R}$ são constantes.

Vamos selecionar algumas exemplos de $p(x)$.

a) Para $p(x)=a x+b$ temos as seguintes soluções

1. $u=C_{1} t^{2}+C_{2} t-\frac{2\left(a C_{1} x+\left(a C_{1}-b C_{3}\right) \ln (a x+b)\right)}{a^{2}}+C_{4}$;

2. $u=C_{1} t^{3}+C_{2} t-\frac{6 t\left(a C_{1} x+\left(a C_{1}-b C_{3}\right) \ln (a x+b)\right)}{a^{2}}+C_{4}$;

3. $u=C_{2} t+\frac{\left(C_{3}+C_{1} t\right) \ln (a x+b)}{a}+C_{4}$;

4. $u=\frac{a\left(a^{2}\left(C_{4}+C_{2} t^{2}\right)+4 C_{1} x-2 a C_{2} x\right)+\left(b\left(-4 C_{1}+2 a C_{2}\right)+a\left(a C_{C 3}+a C_{1} t^{2}-2 C_{1} x\right)\right) \ln (b+a x)}{a^{3}}$.

b) Para $p(x)=a e^{b x}$ temos as seguintes soluções

1. $u=t\left(C_{2}+C_{1} t\right)+C_{4}+\frac{2 e^{-b x}\left(C_{1}+b C_{3}+b C_{1} x\right)}{a b^{2}} ;$

2. $u=C_{4}+t\left(C_{2}+C_{1} t^{2}+\frac{6 e^{-b x}\left(C_{1}+b C_{3}+b C_{1} x\right)}{a b^{2}}\right)$;

3. $u=C_{4}+C_{2} t-\frac{e^{-b x}\left(C_{3}+C_{1} t\right)}{a b}$;

4. $u=C_{4}+\frac{C_{1} e^{-2 b x}}{a^{2} b^{3}}+C_{2} t^{2}+\frac{e^{-b x}\left(-b\left(C_{3}+C_{1} t^{2}\right)+2\left(C_{2}+b C_{2} x\right)\right)}{a b^{2}}$.

Equação 11 Fazendo $f(x)=p(x), g(x)=0, h(x)=0, k(x)=-1$, onde $p(x)$ é uma função de classe $C^{1}$, obtemos a seguinte equação:

$$
p(x) \frac{\partial^{2} u}{\partial t^{2}}=-\frac{\partial^{2} u}{\partial x^{2}} .
$$


Temos as seguintes soluções particulares

- $u=C_{1} x t+C_{2} t+C_{3} x+C_{4}$;

- $u=C_{1} t^{2}+C_{2} x t+C_{3} t+C_{4} x-2 C_{1} \int_{0}^{x}(x-y) p(y) d y+C_{5}$;

- $u=C_{1} t^{3}+C_{2} x t+C_{3} t+C_{4} x-6 C_{1} t \int_{0}^{x}(x-y) p(y) d y+C_{5}$;

- $u=\left(C_{1} x+C_{2}\right) t^{2}+C_{3} x t+C_{4} t+C_{5} x-2 \int_{0}^{x}(x-y)\left(C_{1} y+C_{2}\right) p(y) d y+C_{6}$,

onde $C_{1}, C_{2}, C_{3}, C_{4}, C_{5}, C_{6} \in \mathbb{R}$ são constantes.

Vamos selecionar algumas exemplos de $p(x)$.

a) Para $f(x)=a x^{2}+b x+c$ temos as seguintes soluções

1. $u=C_{4}+C_{2} t+C_{3} x+C_{1} t x$;

2. $u=C_{5}+C_{3} t+\left(C_{4}+C_{2} t\right) x+\frac{1}{6} C_{1}\left(6 t^{2}-x^{2}(6 c+x(2 b+a x))\right)$;

3. $u=C_{5}+C_{3} t+\left(C_{4}+C_{2} t\right) x+\frac{1}{2} C_{1} t\left(2 t^{2}-x^{2}(6 c+x(2 b+a x))\right)$;

4. $u=C_{5}+C_{4} t+C_{5} x+C_{3} t x+t^{2}\left(C_{2}+C_{1} x\right)-$ $\frac{1}{30} x^{2}\left(10 c\left(3 C_{2}+C_{1} x\right)+x\left(5 b\left(2 C_{2}+C_{1} x\right)+a x\left(5 C_{2}+3 C_{1} x\right)\right)\right)$.

b) Para $f(x)=a x^{3}+b x^{2}+c x+d$ temos as seguintes soluções

1. $u=u=C_{4}+C_{2} t+C_{3} x+C_{1} t x$;

2. $u=C_{5}+C_{3} t+C_{1} t^{2}+C_{4} x+C_{2} t x-\frac{1}{30} C_{1} x^{2}(30 d+x(10 c+x(5 b+3 a x)))$;

3. $u=C_{5}+C_{3} t+C_{1} t^{3}+C_{4} x+C_{2} t x-\frac{1}{10} C_{1} t x^{2}(30 d+x(10 c+x(5 b+3 a x)))$;

4. $u=C_{5}+C_{4} t+C_{5} x+C_{3} t x+t^{2}\left(C_{2}+C_{1} x\right)-$ $\frac{1}{30} x^{2}\left(30 C_{2} d+10\left(c C_{2}+C_{1} d\right) x+5\left(c C_{1}+b C_{2}\right) x^{2}+3\left(b C_{1}+a C_{2}\right) x^{3}+2 a C_{1} x^{4}\right)$.

c) Para $f(x)=a \cos (b x)$ temos as seguintes soluções

1. $u=C_{4}+t\left(C_{2}+C_{1} t\right)+\frac{2 e^{-b x}\left(C_{1}+b C_{3}+b C_{1} x\right)}{a b^{2}} ;$ 
2. $u=C_{5}+C_{3} t+C_{1} t^{2}+C_{4} x+C_{2} t x+\frac{2 a C_{1}(-1+\cos (b x))}{b^{2}}$;

3. $u=C_{5}+C_{3} t+C_{1} t^{3}+C_{4} x+C_{2} t x+\frac{6 a C_{1} t(-1+\cos (b x))}{b^{2}}$;

4. $u=C_{5}+C_{4} t+C_{5} x+C_{3} t x+t^{2}\left(C_{2}+C_{1} x\right)+\frac{2 a\left(-b C_{2}+b C_{1} x+b\left(C_{2}+C_{1} x\right) \cos (b x)-2 C_{1} \sin (b x)\right)}{b^{3}}$.

\subsubsection{Soluções numéricas}

Equação 12 Para a equação:

$$
f \frac{\partial^{2} u}{\partial t^{2}}+g \frac{\partial u}{\partial t}+h u=\frac{\partial}{\partial x}\left(k \frac{\partial u}{\partial x}\right)
$$

selecionamos os seguintes conjuntos de parâmetros para a equação:

a) $\quad f(x)=\frac{x^{4}+x^{3}+x^{2}+x+1}{3}$

- $g(x)=\frac{x^{3}+x^{2}+x+1}{3}$

- $h(x)=\frac{x+1}{3}$

- $k(x)=\frac{x^{4}+x^{3}+x^{2}+x+1}{3}$,

b) $\quad f(x)=\frac{-x^{4}+x^{3}-x^{2}+x+1}{3}$

- $g(x)=\frac{-x^{3}+x^{2}-x+1}{3}$

- $h(x)=\frac{x+1}{3}$

- $k(x)=\frac{-x^{4}+x^{3}-x^{2}+x+1}{3}$,

c) $\quad f(x)=e^{-x}$

- $g(x)=e^{-x}$

- $h(x)=e^{-x}$

- $k(x)=e^{-x}$.

Para determinar as soluções numéricas da equação (6.12) é necessário impor condições iniciais e de contorno. Como a equação é de segundo grau em $t$ e $x$ devemos impor duas condições para cada variável. Aplicamos as seguintes condições iniciais e de contorno.

$$
\begin{aligned}
& u(x, 0)=l_{1}(x) \\
& \frac{\partial u(x, 0)}{\partial t}=l_{2}(x) \\
& \frac{\partial u(0, t)}{\partial x}=m_{1}(t) \\
& \frac{\partial u(L, t)}{\partial x}=m_{2}(t) .
\end{aligned}
$$


Também devemos aplicar as seguintes condições de compatibilidade.

$$
\begin{aligned}
& m_{1}(0)=l_{1}^{\prime}(0) \\
& m_{2}(0)=l_{1}^{\prime}(L) .
\end{aligned}
$$

Para resolver a equação (6.12) com as condições (6.13), (6.14) e (6.15), foi utilizado o método das linhas [Sch91], [SG09]. O método consiste, basicamente, em discretizar as derivadas espaciais usando diferenças finitas. Feito isso, a equação diferencial parcial é aproximada por um sistema de equações diferenciais ordinárias. Esse sistema pode ser resolvido por algum método para soluções de sistemas de EDOs. Nesse trabalho foi escolhido o Runge-Kutta de quarta ordem.

Primeiro a equação (5.1) é descomposta num sistema de equações com derivadas de primeira ordem no tempo. Fazendo $\frac{\partial u}{\partial t}=v$, temos:

$$
\left\{\begin{array}{l}
\frac{\partial u}{\partial t}=v \\
\frac{\partial v}{\partial t}=\frac{1}{f(x)}\left(-g(x) v-h(x) u+\frac{\partial}{\partial x}\left(k(x) \frac{\partial u}{\partial x}\right)\right)
\end{array}\right.
$$

Expandindo as derivadas em $\mathrm{x}$ temos:

$$
\left\{\begin{array}{l}
\frac{\partial u}{\partial t}=v \\
\frac{\partial v}{\partial t}=\frac{1}{f(x)}\left(-g(x) v-h(x) u+k^{\prime}(x) \frac{\partial u}{\partial x}+k(x) \frac{\partial^{2} u}{\partial x^{2}}\right)
\end{array}\right.
$$

Dividindo o intervalo $[0, L]$ em um grade de $M+1$ pontos temos $\Delta x=L / M$. Se o índice $i$ representa a posição na grade, temos $x_{i}=i \Delta x, u_{i}=u\left(x_{i}, t\right), \frac{\partial u_{i}}{\partial x}=\frac{\partial u}{\partial x}\left(x_{i}, t\right)$ e $\frac{\partial^{2} u_{i}}{\partial x^{2}}=\frac{\partial^{2} u}{\partial x^{2}}\left(x_{i}, t\right)$. Assim, podemos aproximar as derivadas espaciais de $u$ pelas suas diferenças finitas:

$$
\begin{gathered}
\frac{\partial u_{i}}{\partial x}=\frac{u_{i+1}-u_{i-1}}{2 \Delta x} \\
\frac{\partial^{2} u_{i}}{\partial x^{2}}=\frac{u_{i+1}-2 u_{i}+u_{i-1}}{(\Delta x)^{2}}
\end{gathered}
$$

Desta maneira o sistema (6.17) fica:

$$
\left\{\begin{array}{l}
\frac{\partial u_{i}}{\partial t}=v_{i} \\
\frac{\partial v_{i}}{\partial t}=\frac{1}{f_{i}}\left(-g_{i} v_{i}-h_{i} u_{i}+k_{i}^{\prime} \frac{u_{i+1}-u_{i-1}}{2 \Delta x}+k_{i} \frac{u_{i+1}-2 u_{i}+u_{i-1}}{(\Delta x)^{2}}\right)
\end{array}\right.
$$

A resolução deste sistema de EDOs nos dará as $\mathrm{M}+1$ soluções $u_{1}(t), u_{2}(t), \ldots, u_{M+1}(t)$ que é uma aproximação da solução da EDP.

Para encontrar as quatro soluções, da equação (6.12), selecionamos quatro grupos de condições iniciais e de contorno. No grupo 1 as funções $l_{1}(x), l_{2}(x), m_{1}(t)$ e $m_{1}(t)$ são polinômios de grau menor ou igual a 5, no grupo 2 as funções são da forma $P(x) / Q(x)$, onde $P(x)$ e $Q(x)$ são polinômios de grau menor ou igual a 5 , no grupo 3 as funções da forma $\sum_{i=1}^{5} a_{i} \sin \left(b_{i} z+c_{i}\right)+d$ e no grupo 4 as funções são da forma $\sum_{i=1}^{5} a_{i} \exp \left(b_{i} z+c_{i}\right)+d$. 


\subsection{Procedimento de simulação}

Nessa seção descreveremos o procedimento utilizado nas simulações de estimação dos coeficientes funcionais da EDP (5.1). O procedimento foi desenvolvido no programa estatístico R, versão 3.2.3, e utilizando os pacotes de utilitários deSolve, RandomFields e pracma.

Selecionamos 12 EDPs, que são casos particulares da EDP (5.1), para aplicar os estimadores nas soluções destas equações. Como descrito na seção 6.1, para as equações 10, 11, 12 selecionamos mais de um conjunto de parâmetros para as simulações, denominados 10a, 10b, 11a, 11b, 11c, 12a, 12b, e 12c. Assim teremos 17 conjuntos de resultados para as simulações.

As soluções, das equações, foram encontradas conforme descrito na seção 6.1. As soluções, numéricas, da equação (6.12) foram encontradas com a utilização do pacote deSolve e aplicando o método das linhas, conforme indicado em [PS10], [CM12], [Blo14].

Na descrição dos estimadores, foi suposto que as soluções disponíveis $u(x, t)$ estão definidas para $x$ e $t$ pertencentes aos intervalos $[0, L]$ e $[0, T]$ respectivamente. Numa aplicação real, as soluções disponíveis seriam aproximadas por um conjunto discreto de pontos $\left\{u\left(x_{i}, t_{j}\right), i=1, \ldots, P, j=1, \ldots, Q\right\}$. Na simulação fizemos $L=T=1, P=Q=101$ e $x_{i+1}-x_{i}=1 / 100, x_{1}=0, t_{j+1}-t_{j}=1 / 100 \mathrm{e}$ $t_{1}=0$. Desta maneira iremos representar os intervalos de $x$ e $t$ por conjuntos com 101 pontos, e cada solução, de uma EDP, por um conjunto com $101^{2}$ pontos sobre a grade $\left\{x_{1}, \ldots, x_{101}\right\} \times\left\{t_{1}, \ldots, t_{101}\right\}$.

Nos estimadores com integral supomos que temos a disposição quatro conjuntos, distintos, de soluções para a EDP, onde as soluções são da forma $v_{i}=u_{i}+\epsilon_{i}$ e $\epsilon_{i}$ é um ruído. Para cada conjunto obtemos uma solução média $\bar{v}^{i}=\frac{\sum v_{i}}{n}$, e utilizamos essas médias nos estimadores. Mas não temos como gerar um número grande de soluções para uma EDP. Como a equação (5.1) é uma EDP linear homogênea, sabemos que qualquer combinação linear de soluções da EDP é também uma solução. Assim, podemos considerar que a média das soluções como sendo uma solução $u^{j}$ mais uma média de ruídos $\bar{\epsilon}^{j}=\frac{\sum \epsilon_{i}}{n}$. Assim, na simulação vamos trabalhar com quatro soluções e somar, a cada uma, uma média de ruídos. Aplicaremos aos estimadores diretos o mesmo procedimento.

Vamos fazer a estimação dos coeficientes de algumas EDPs por dois grupos de estimadores. Para que possamos fazer uma comparação dos resultados precisamos que esses resultados sejam independentes da realização dos ruídos. Para que isso seja possível precisamos que os ruídos utilizados sejam os mesmos em todas as simulações. Assim iremos gerar um conjunto de realizações para cada um dos geradores de ruídos descritos no capítulo 4, que serão utilizados em todas as simulações.

Para medir o desempenho dos estimadores vamos utilizar os erros quadráticos descritos a seguir.

$$
\begin{aligned}
\|\hat{f}-f\|_{2}^{2} & =\int_{0}^{L}(\hat{f}(x)-f(x))^{2} d x \\
\|\hat{g}-g\|_{2}^{2} & =\int_{0}^{L}(\hat{g}(x)-g(x))^{2} d x
\end{aligned}
$$




$$
\begin{aligned}
\|\hat{h}-h\|_{2}^{2} & =\int_{0}^{L}(\hat{h}(x)-h(x))^{2} d x \\
\|\hat{k}-k\|_{2}^{2} & =\int_{0}^{L}(\hat{k}(x)-k(x))^{2} d x
\end{aligned}
$$

\subsubsection{Procedimento de simulação}

Passo 1 - Gerar e armazenar os conjuntos de realizações dos ruídos que serão utilizados. Iremos gerar quatro conjuntos de realizações de ruídos para cada um dos três geradores de ruídos descritos no capítulo 4, um total de 12 conjuntos. Cada um dos conjuntos terá 1000 realizações.

Passo 2 - Gerar e armazenar os conjuntos que representam as quatros soluções, necessárias para os estimadores, para cada uma das EDPs selecionadas. Para as EDPs de 1 a 11c temos soluções analíticas, e assim apenas calculamos os valores da soluções para $x_{i}$ e $t_{j}$ pertencentes os conjuntos que representam os intervalos de $x$ e $t$. No caso da equação (6.12) encontramos as soluções para a região representada por $\left\{x_{1}, \ldots, x_{N+1}\right\} \times\left\{t_{1}, \ldots, t_{N+1}\right\}$. Os coeficientes das equações e soluções, utilizadas nas simulações, foram escolhidos de forma que as soluções fossem suaves.

Passo 3 - Fazer as estimativas dos coeficientes, das EDPs selecionadas, aplicando os estimadores com integral e diretos. Nessas estimativas utilizaremos as soluções e ruídos gerados nos passos 1 e 2. Iremos aplicar os estimadores em soluções sem ruído, com o acréscimo de uma realização de ruído, e para soluções com acréscimo de médias de ruídos. As médias, utilizadas, serão de 10,20, .., 1000 ruídos. Assim teremos 102 estimativas para cada coeficiente das equações selecionadas.

Passo 4 - Calcular e armazenar os erros quadráticos de cada uma das estimativas realizadas no item 3, utilizando as expressões (6.19) a (6.21).

\section{Procedimento para os estimadores com integrais}

Passo 1 - Calcular os valores de $\alpha_{j}, \beta_{j}$ e $\gamma_{j}$ pelas expressões (5.4), (5.5) e (5.6), para cada $x_{i}$ do conjunto que representa o intervalo $[0,1]$. O cálculo de $\alpha_{j}, \beta_{j}$ e $\gamma_{j}$ envolve o cálculo de uma integral de uma função em $t$ no intervalo $[0,1]$, mas temos uma conjunto discreto que representa esse intervalo, e assim, temos um conjunto discreto, que representa a função, a ser integrada. Para o cálculo da integral vamos passar uma spline cúbica, pelos pontos do conjunto que representa a função, para obter uma aproximação, e desta maneira calcular a integral. Fazemos isso utilizando a função splinefun, para gerar a spline. Não foi possível utilizar a função integrete do R no cálculo da integral, pois, como descrito em [Blo14], essa função tem dificuldade em integrar funções que oscilam muito, que é o nosso caso devido ao ruído. Assim, utilizamos a função integral da biblioteca pracma, que implementa outros métodos de 
integração, por exemplo o Gauss-Kronrod, que utilizamos para calcular a integral.

Passo 2 - Calcular a primeira e segunda derivadas de $\gamma_{j}\left(\gamma_{j}^{\prime}\right.$ e $\left.\gamma_{j}^{\prime \prime}\right)$. Para isso passamos uma spline cúbica pelos pontos de $\gamma_{j}$, obtendo uma aproximação que é utilizada no cálculo das derivadas. A função splinefun, utilizada para encontrar a spline, permite o cálculo das derivadas.

Passo 3 - Calcular os determinantes

$$
\operatorname{det}\left(\alpha, \beta, \gamma, \gamma^{\prime \prime}\right), \operatorname{det}\left(\gamma^{\prime \prime}, \beta, \gamma, \gamma^{\prime}\right), \operatorname{det}\left(\alpha, \gamma^{\prime \prime}, \gamma, \gamma^{\prime}\right), \operatorname{det}\left(\alpha, \beta, \gamma^{\prime \prime}, \gamma^{\prime}\right) \operatorname{e} \operatorname{det}\left(\alpha, \beta, \gamma, \gamma^{\prime}\right) \text {, }
$$

utilizados nas expressões (5.8), (5.9), (5.10) e (5.11), para cada $x_{i}$ do conjunto que representa o intervalo $[0,1]$.

Passo 4 - Calcular os valores estimados de $k\left(x_{i}\right)$ pela expressão (5.8). A integral é calculada pelo mesmo procedimento descrito no passo 1.

Passo 5 - Estimar os valores de $f\left(x_{i}\right), g\left(x_{i}\right)$ e $h\left(x_{i}\right)$ utilizando as expressões (5.9), (5.10), (5.11) e os valores de $k\left(x_{i}\right)$ calculados no passo 4 .

Passo 6 - Calcular e armazenar os erros quadráticos, das estimativas de $k\left(x_{i}\right), f\left(x_{i}\right), g\left(x_{i}\right)$ e $h\left(x_{i}\right)$, pelas expressões, (6.19) a (6.22). O cálculo das integrais é feito como descrito no passo 1.

\section{Procedimento para os estimadores diretos}

Passo 1 - Calcular as derivadas

$$
\frac{\partial^{2} u_{j}}{\partial t^{2}}, \frac{\partial u_{j}}{\partial t}, \frac{\partial^{2} u_{j}}{\partial x^{2}} \text { e } \frac{\partial u_{j}}{\partial x}
$$

das soluções utilizadas no estimador. Usamos o mesmo procedimento do cálculo das derivadas de $\gamma_{j}$. Nesse caso passamos uma spline mantendo fixo o valor de $x$ e outra fixando o valor de $t$. Vimos no capítulo 5 que é necessário fixar um valor de $t$ para aplicar os estimadores. Na nossa simulação usamos o valor de $t=t_{50}=0,49$. As derivadas são calculadas para todos os valores de $x_{i}$ e $t_{50}$.

Passo 2 - Calcular os valores dos determinantes

$$
\begin{gathered}
\operatorname{det}\left(\frac{\partial^{2} U}{\partial t^{2}}, \frac{\partial U}{\partial t}, U, \frac{\partial^{2} U}{\partial x^{2}}\right), \operatorname{det}\left(\frac{\partial^{2} U}{\partial x^{2}}, \frac{\partial U}{\partial t}, U, \frac{\partial U}{\partial x}\right), \operatorname{det}\left(\frac{\partial^{2} U}{\partial t^{2}}, \frac{\partial^{2} U}{\partial x^{2}}, U, \frac{\partial U}{\partial x}\right), \\
\operatorname{det}\left(\frac{\partial^{2} U}{\partial t^{2}}, \frac{\partial U}{\partial t}, \frac{\partial^{2} U}{\partial x^{2}}, \frac{\partial U}{\partial x}\right) \text { e } \operatorname{det}\left(\frac{\partial^{2} U}{\partial t^{2}}, \frac{\partial U}{\partial t}, U, \frac{\partial U}{\partial x}\right)
\end{gathered}
$$


para cada $x_{i}$

Passo 3 - Calcular os valores estimados de $k\left(x_{i}\right)$ pela expressão (5.12). A integral é calculada da mesma maneira descrita no passo 1 de 6.2.1.

Passo 4 - Estimar os valores de $f\left(x_{i}\right), g\left(x_{i}\right)$ e $h\left(x_{i}\right)$ utilizando as expressões $(5.13),(5.14),(5.15)$ e os valores de $k\left(x_{i}\right)$ calculados no passo 3 .

Passo 5 - Calcular as diferença entre o estimado e o real pelas expressões, (6.19) a (6.22). O cálculo das integrais é feito como descrito no passo 2 do procedimento para estimadores com integral.

\subsection{Análise dos resultados}

Na seção 6.1 apresentamos as equações selecionadas e as suas soluções. Para as equações (6.4), (6.5), (6.6), (6.7), (6.8) e (6.9) apresentamos mais soluções que as quatro necessárias para a aplicação dos estimadores. A seguir temos um tabela com as equações e as soluções utilizadas nas simulações. Para a equação (6.12) só geramos as quatro soluções, necessárias, para cada um dos conjuntos de coeficientes.

$\begin{array}{ll}\begin{array}{l}\text { Equação } \\ (6.1)\end{array} & \begin{array}{l}\text { Soluções } \\ (6.2)\end{array} \\ 1,2,3,4 \\ (6.3) & 1,2,3,4 \\ (6.4) & 1,3,3,4 \\ (6.5) & 1,3,5,6 \\ (6.6) & 1,3,5,7 \\ (6.7) & 1,2,3,4 \\ (6.8) & 1,2,3,4 \\ (6.9) & 1,2,3,4 \\ (6.10 \mathrm{a}) & 1,2,3,4 \\ (6.10 \mathrm{~b}) & 1,2,3,4 \\ (6.11 \mathrm{a}) & 1,2,3,4 \\ (6.11 \mathrm{~b}) & 1,2,3,4 \\ (6.11 \mathrm{c}) & 1,2,3,4\end{array}$

Tabela 6.1: Soluções selecionadas por equação

Para não

\subsubsection{Seleção das soluções}

Analisando as expressões dos dois grupos de estimadores vemos que pode haver problema de divisão por zero. Pelas expressões (5.8) a (5.11) vemos que caso o determinante $\operatorname{det}\left(\alpha, \beta, \gamma, \gamma^{\prime}\right)$ resulte em zero para algum ponto do intervalo $[0, L]$ ocorrerá divisão por zero. Nos estimadores diretos teremos o mesmo problema para o determinante $\operatorname{det}\left(\frac{\partial^{2} U}{\partial t^{2}}, \frac{\partial U}{\partial t}, U, \frac{\partial U}{\partial x}\right)$ conforme as expressões (5.12) a (5.15). Naturalmente como trabalhamos com um conjunto discreto, como aproximação do 
intervalo $[0, L]$, dificilmente haverá uma divisão por zero. Mas pode ocorrer uma divisão por valores muito próximos de zero, que podem gerar pontos muito distantes da curva real, comprometendo as estimativas dos coeficientes. Podemos ver isso nas equações (6.4), (6.5), (6.6), (6.7), (6.10a), (6.12a) e (6.12c). Nas equações (6.5), (6.6), e (6.7) não foi possível aplicar os estimadores para qualquer grupo de quatro soluções. Para alguns grupos, os estimadores geraram erros devido a utilização de valores muito grandes em algumas operações ou no cálculo de integrais. Na equação (6.4), com as soluções 1, 2, 4 e 5, embora os determinantes se anulassem em um ponto do intervalo $[0, L]$, não ocorreram erros devidos a operação com valores muito grandes. Mas o resultado da estimação apresentou valores muito distantes dos reais. Podemos ver isso nos gráficos 6.1, 6.2 e 6.3. Para as soluções 1, 3, 5, 6 os resultados são muito melhores, como podemos ver nos gráficos 6.4, 6.5 e 6.6, pois os determinantes não se anulam em nenhum ponto do intervalo $[0, L]$.
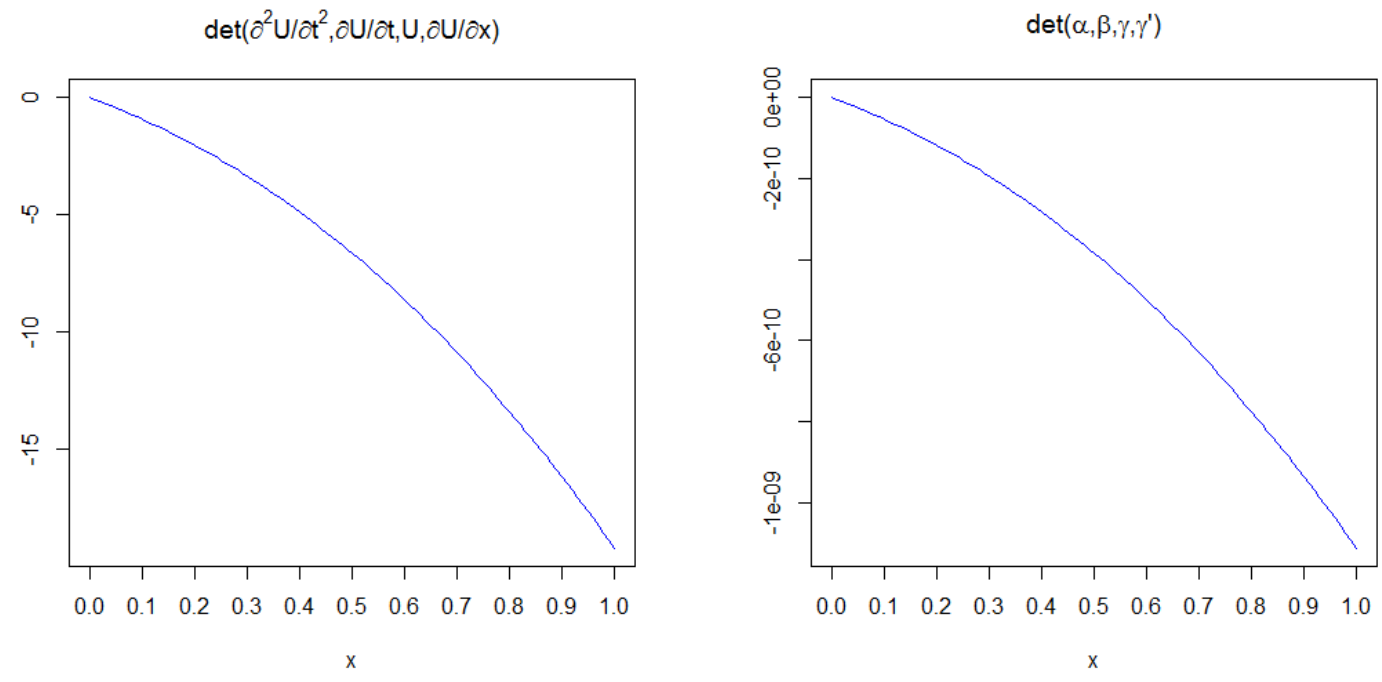

Figura 6.1: Determinante utilizado nos estimadores da equação (6.4) para as soluções 1, 2, 4 e 5
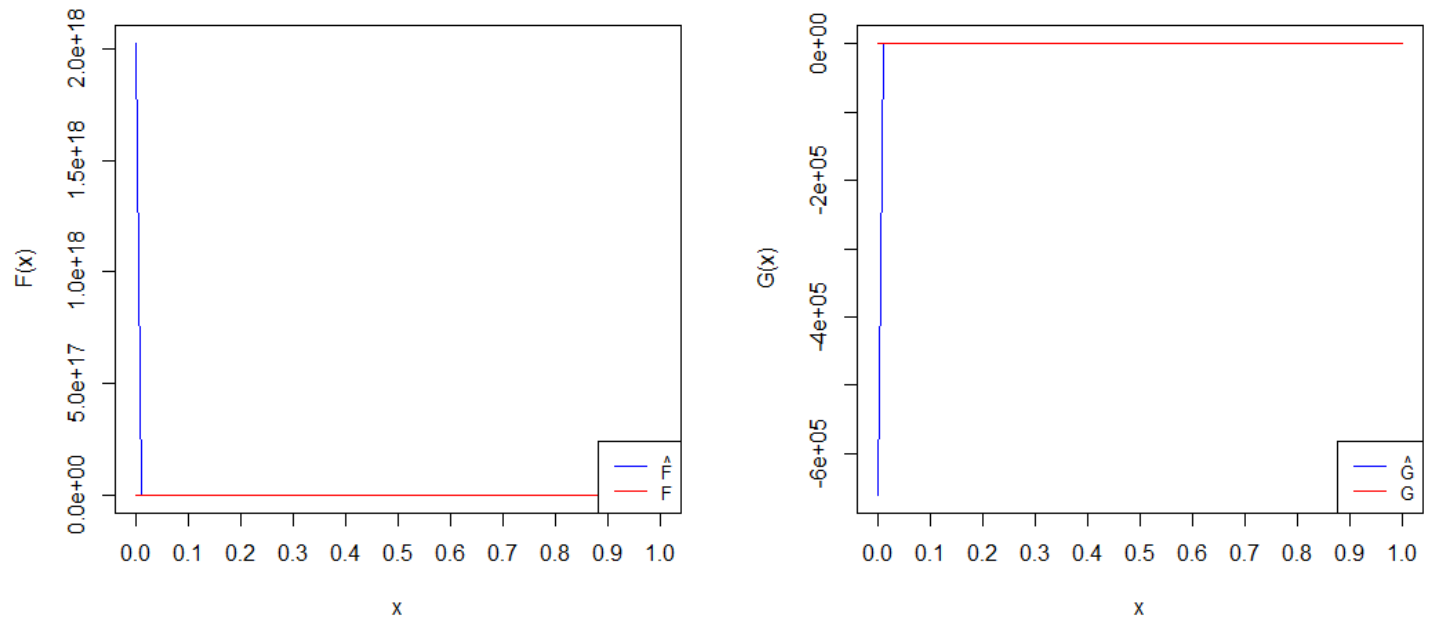

Figura 6.2: Estimativa funções $F$ e $G$ da equação (6.4) com estimadores diretos para as soluções 1, 2, 4 e 5 

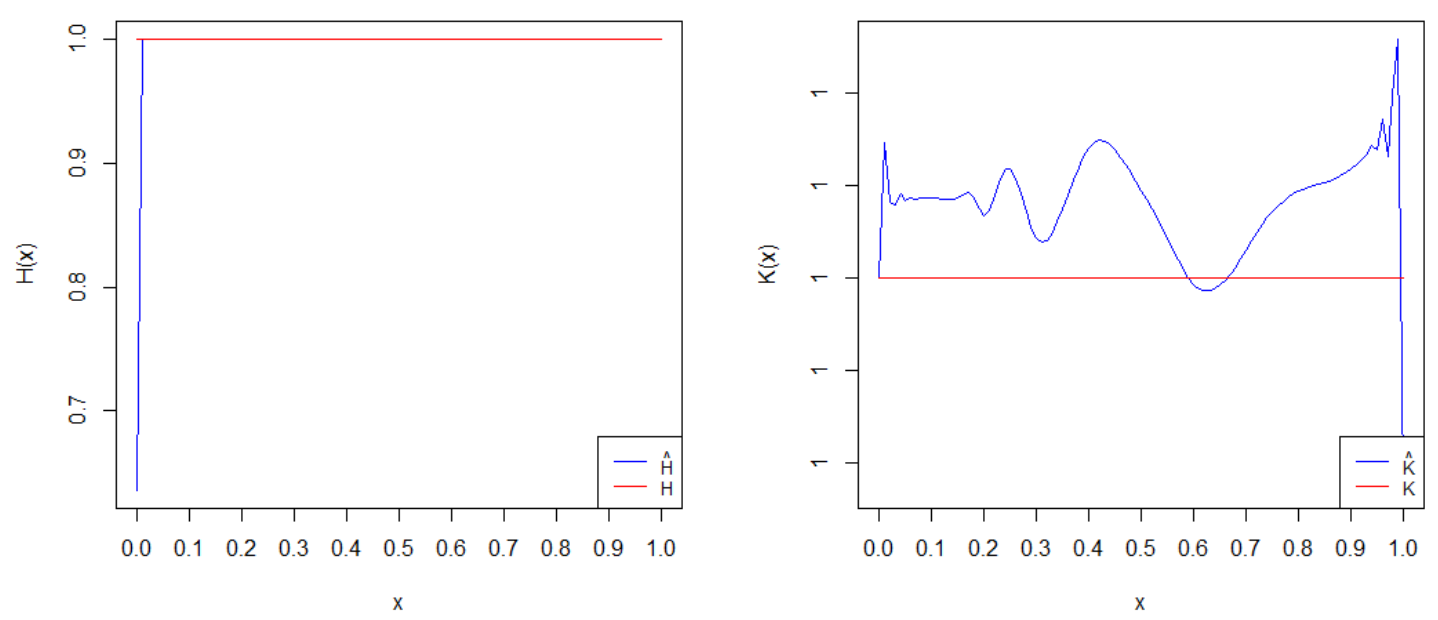

Figura 6.3: Estimativa funções $H$ e $K$ da equação (6.4) com estimadores diretos para as soluções 1, 2, 4 e 5
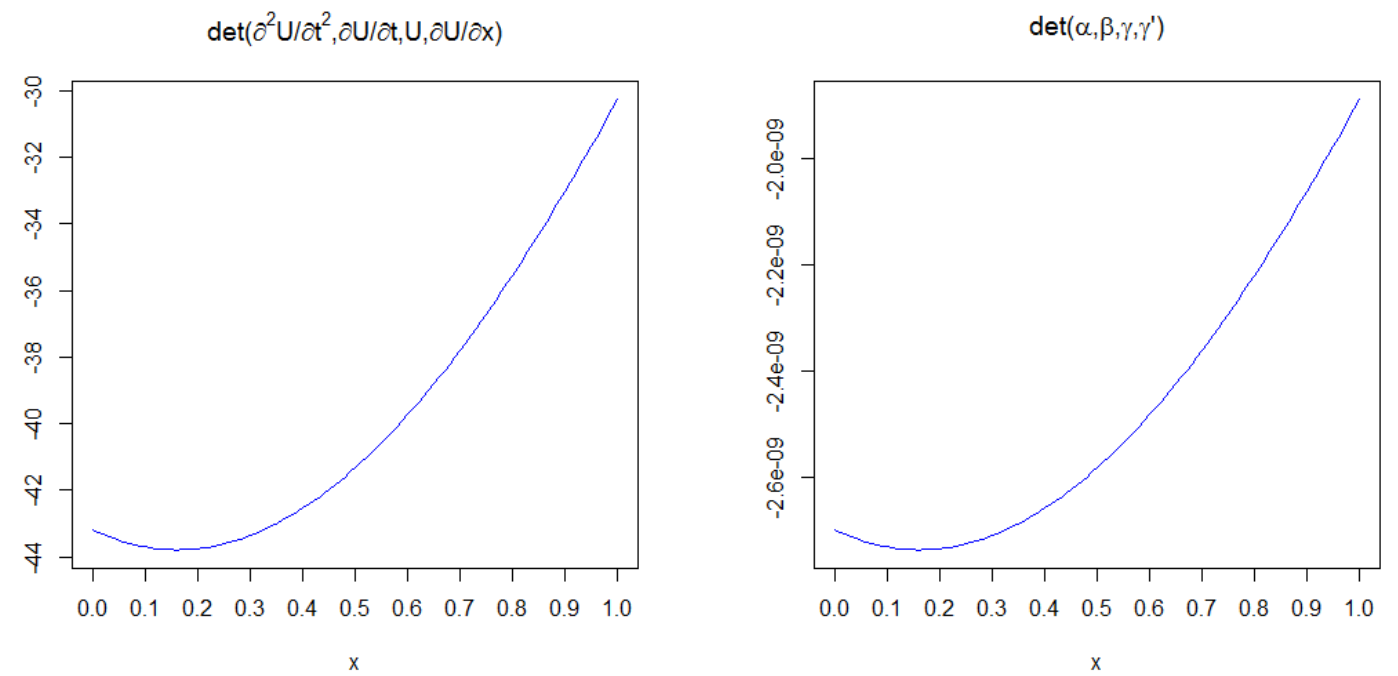

Figura 6.4: Determinante utilizado nos estimadores da equação (6.4) para as soluções 1, 3, 5, 6 

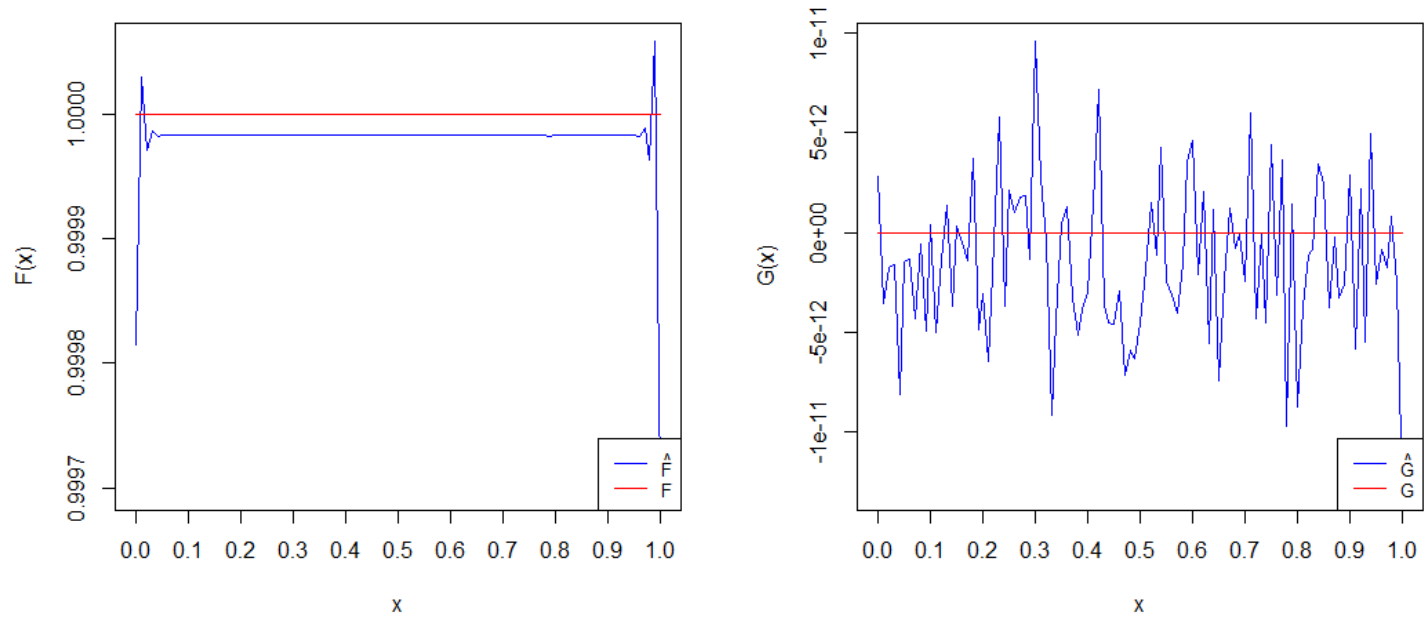

Figura 6.5: Estimativa funções $F$ e $G$ da equação (6.4) com estimadores diretos para as soluções 1, 3, 5, 6
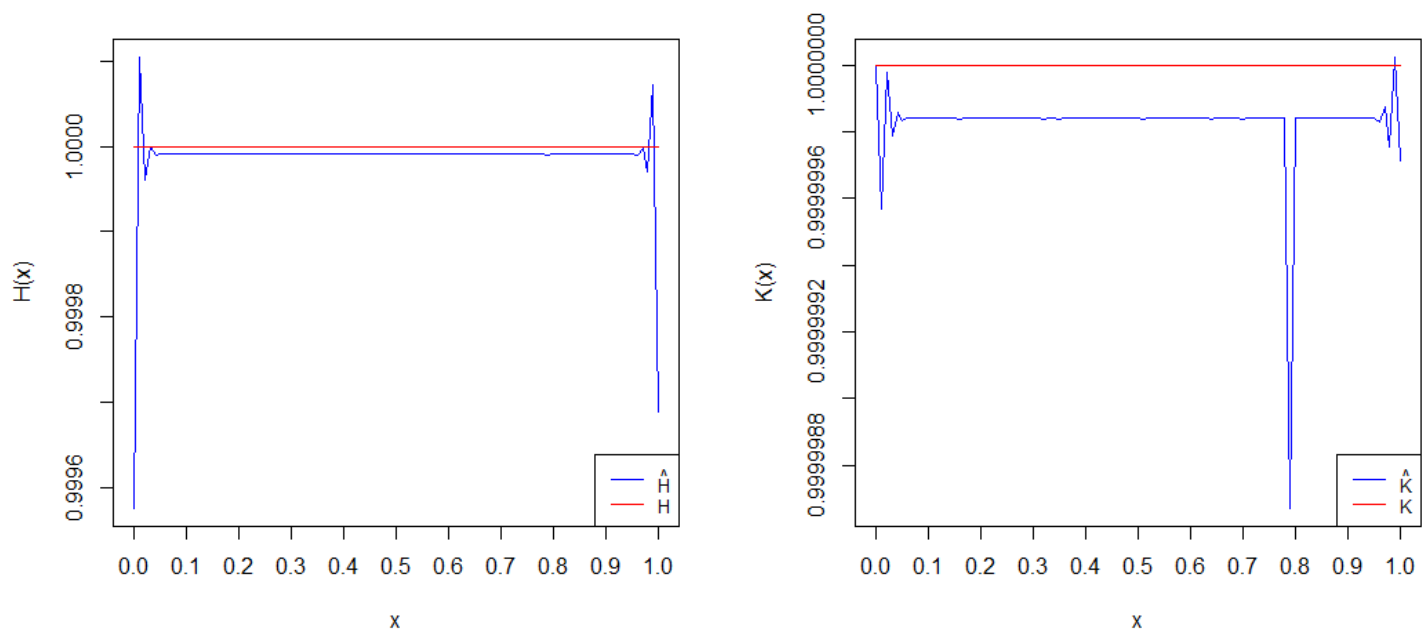

Figura 6.6: Estimativa funções $H$ e $K$ da equação (6.4) com estimadores diretos para as soluções 1, 3, 5, 6

$\mathrm{Na}$ equação (6.12a) foi possível aplicar os estimadores, embora os determinantes se anulem em um ponto do intervalo [0,1], como é possível ver nos gráficos 6.7. A seguir temos os gráficos para a equação (6.12a). 

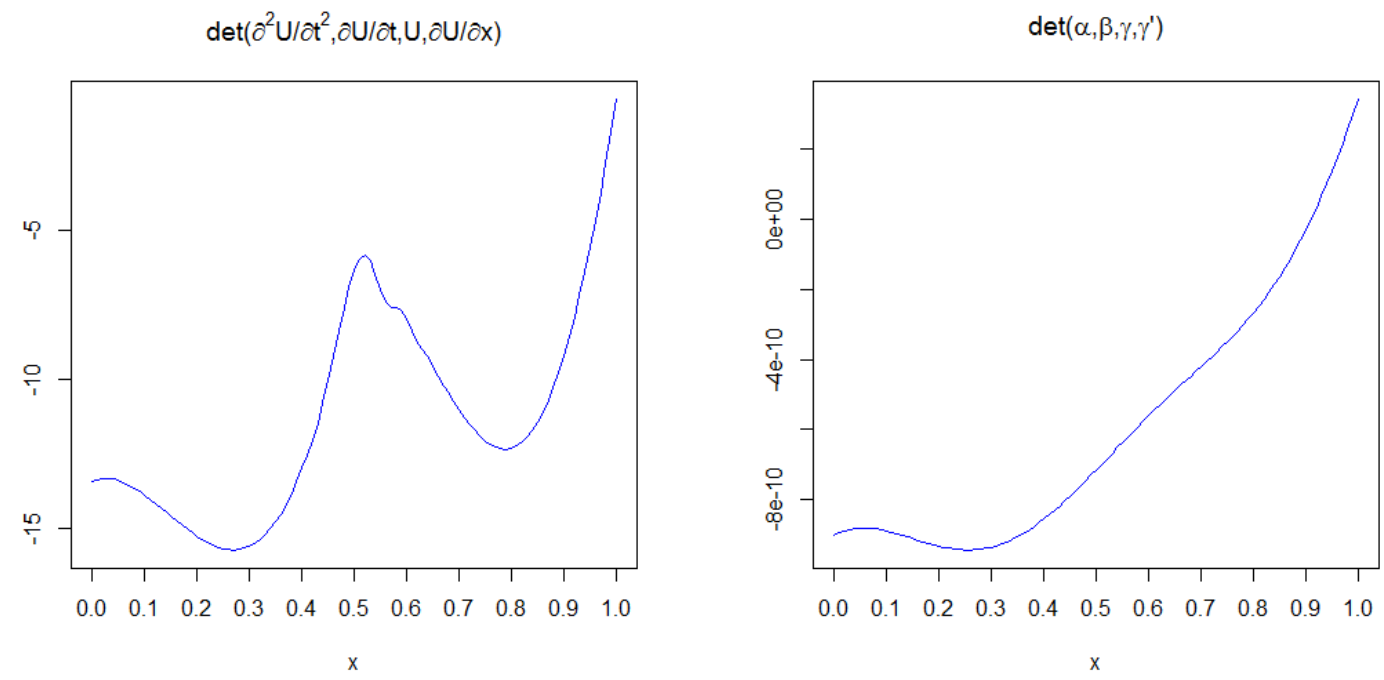

Figura 6.7: Determinante utilizado nos estimadores da equação (6.12a)
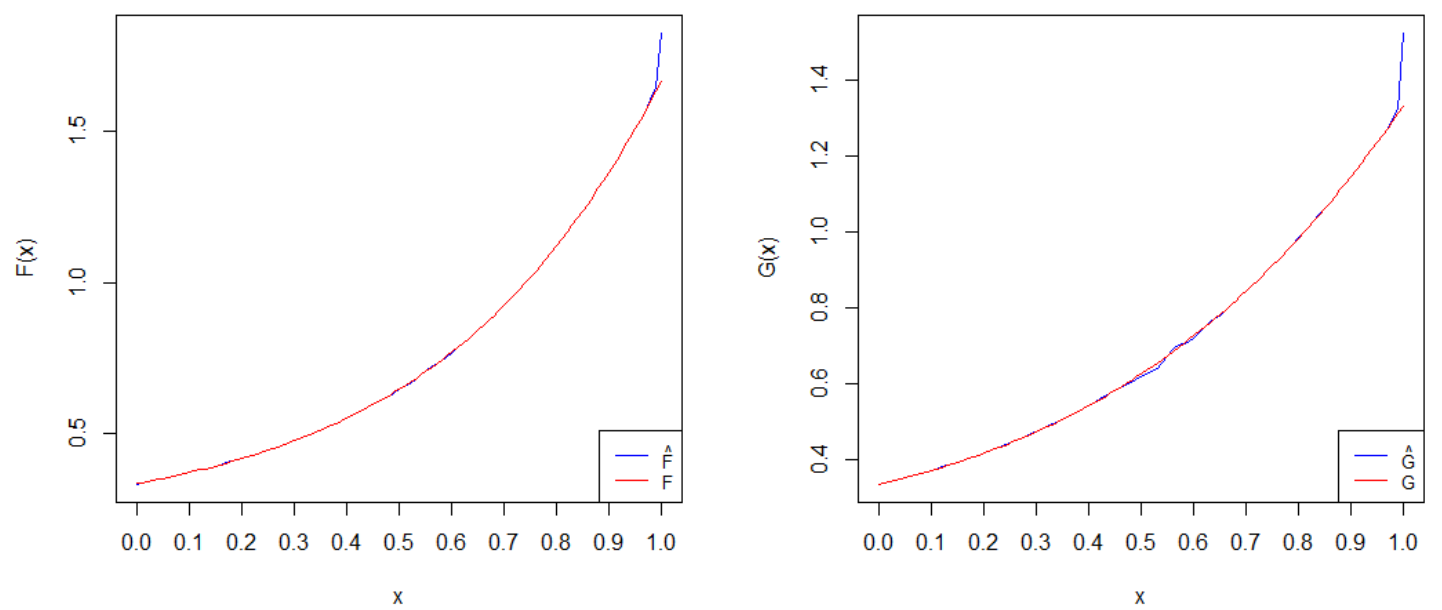

Figura 6.8: Estimativa funções $F$ e $G$ da equação (6.12a) 

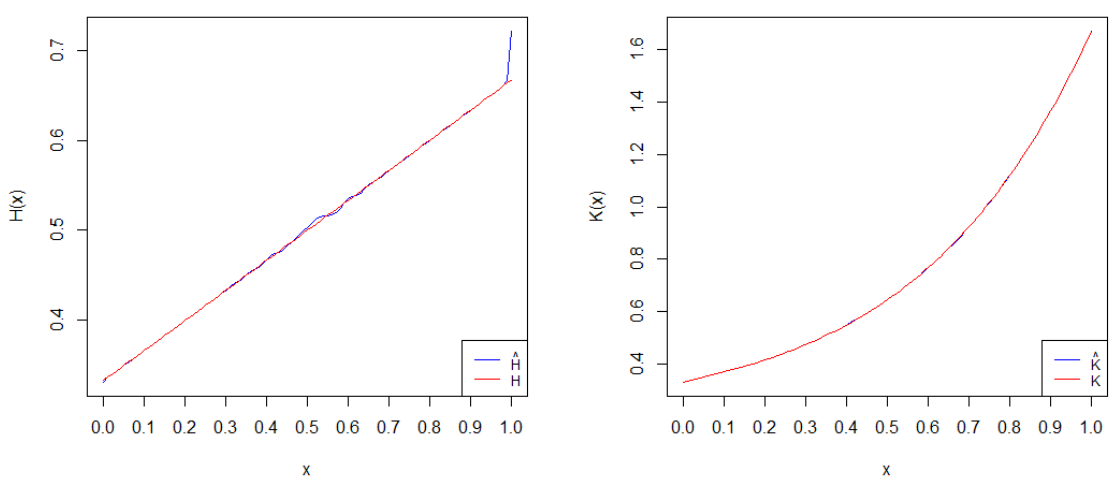

Figura 6.9: Estimativa funções $H$ e $K$ da equação (6.12a)

Assim, vemos que a seleção das soluções utilizadas, tem impacto na estimativa dos coeficientes. Mesmo que as soluções sejam linearmente independentes, os determinantes $\operatorname{det}\left(\alpha, \beta, \gamma, \gamma^{\prime}\right) \mathrm{e}$ $\operatorname{det}\left(\frac{\partial^{2} U}{\partial t^{2}}, \frac{\partial U}{\partial t}, U, \frac{\partial U}{\partial x}\right)$ podem ser nulos em algum ponto do intervalo $[0, L]$, e inviabilizar a aplicação dos estimadores ou gerar estimativas muito ruins.

\subsubsection{Comparação entre os estimadores}

As tabelas 6.10 a 6.20 apresentam os valores dos erros quadráticos calculados para as equações selecionadas e os dois grupos de estimadores. Temos tabelas para soluções sem ruído, para cada um dos tipos de ruído e soluções com médias de 1, 100 e 1000 ruídos. Nas tabelas 6.3 a 6.9 apresentamos as médias dos erros quadráticos. Para facilitar a comparação e visualizar as diferenças entre os estimadores geramos os gráficos 6.10 a 6.27. Esses gráficos representam, através de barras, o percentual da diferença dos erros quadráticos entre os estimadores diretos e os com integral, em relação aos erros quadráticos dos estimadores com integral. Sendo $\operatorname{Err}_{\text {Dir }}$ o erro quadrático do estimador direto, e $\operatorname{Err}_{\text {Int }}$ o erro quadrático do estimador com integral, o tamanho da barra é calculado com tam $=\left(\left(E r r_{\text {Dir }}-E r r_{\text {Int }}\right) / E r r_{\text {Int }}\right) 100$. Assim, um valor positivo indica que o estimador direto apresentou erros maior que o com integral, e valor negativo o contrário. Para facilitar a visualização, valores maiores que 100 e menores que -100, foram truncados para 100 e -100 respectivamente.

Analisando o gráfico 6.10 não é possível notar se um dos dois grupos de estimadores apresenta um desempenho superior ao outro. Mas pelas duas primeiras linhas da tabela 6.3 vemos que o desempenho dos estimadores diretos é, em média, melhor que os com integral, quando consideramos todas as equações. Analisando as quatro últimas linhas, da tabela, vemos que os dois conjuntos de estimadores apresentam desempenhos equivalentes, em média, se consideramos as equações que possuem soluções analíticas (equações 1 a 11c). Quando comparamos os desempenhos para equações com soluções numéricas (equações 12a a 12c), vemos que os estimadores diretos têm um desempenho melhor que os com integrais. Devemos observar que as soluções numéricas são aproximações das soluções exatas, e assim possuem erros não aleatórios, o que geram estimativas com erros maiores que as das equações com soluções exatas. Nas expressões dos estimadores com integral, temos integrais, envolvendo as soluções, ao longo do intervalo $[0, T]$. Assim, toda a informação contida nas soluções é utilizada nas estimativas dos coeficientes. Assim todo erro, relativo a aproximação 
da solução, é acumulado. Já nos estimadores diretos só é utilizada a informação contida, apenas, em uma curva sobre a solução em que $t$ é constante. Desta maneira o acumulo de erro é menor.

Como indicado nos capítulos 5 e na seção 6.2, necessitamos escolher um valor de $t$ para aplicarmos os estimadores diretos. Utilizamos $t=t_{50}=0,49$ para as nossas simulações. Mas haveria diferença na escolha do valor de $t$ utilizado? Nos gráficos 6.10, 6.11 e 6.12 apresentamos as diferenças entre os estimadores diretos e com integral, para soluções sem ruídos, para as escolhas de $t=t_{50}=0,49$, e $t=t_{25}=0,24$ e $t=t_{75}=0,74$, respectivamente. Podemos observar que a escolha do valor de $t$ interfere nos resultados dos estimadores diretos. As diferenças entre os erros quadráticos podem ser significativas conforme as tabelas 6.2 e 6.11. Analisando os gráficos 6.13, 6.14, 6.15, 6.18, 6.19, 6.20, $6.23,6.24$ e 6.25 vemos que o impacto da escolha do valor de $t$, utilizado nos estimadores diretos, não é significativo na presença de ruído. Para comparação entre os dois grupos de estimadores vamos trabalhar com $t=t_{50}=0,49$. Já para os estimadores com integral não há necessidade de fixarmos um valor de $t$.

Analisando as tabelas 6.12, 6.15 e 6.18 vemos valores de erros quadráticos(em negrito) muito elevados. Esses valores são resultado do problema descrito no início deste capítulo, os determinantes $\operatorname{det}\left(\alpha, \beta, \gamma, \gamma^{\prime}\right)$ e $\operatorname{det}\left(\frac{\partial^{2} U}{\partial t^{2}}, \frac{\partial U}{\partial t}, U, \frac{\partial U}{\partial x}\right)$ se anulam em pelo mesmo um ponto do intervalo $[0, L]$. Desta maneira a comparação dos desempenhos dos estimadores fica comprometida. As médias envolvendo todos os valores são apresentadas nas tabelas 6.4, 6.5 e 6.6. Nas tabelas 6.7, 6.8 e 6.9 eliminamos as equações que apresentaram problemas. Esses problemas ocorreram nas equações (6.3), (6.10b) e (6.12a) com o ruído 1, (6.6), (6.7), (6.10b), (6.11a), (6.11b) e (6.11c) com o ruído 2, e (6.6), (6.7), (6.10b), (6.11a), (6.11b), (6.11c), (6.12a) e (6.12c) com o ruído 3.

Analisando os gráficos 6.12 a 6.20, vemos claramente que, na presença de ruído, os estimadores com integral tem um desempenho melhor que os estimadores diretos, principalmente para as equações com soluções analíticas. Nas equações com soluções numéricas vemos que o mesmo problema descrito anteriormente. Isso pode ser comprovado pelas tabelas 6.7, 6.8 e 6.9. Numa situação prática as soluções disponíveis, embora numéricas, não teriam erros de aproximação, apenas erros devido a ruídos.

\subsubsection{Comparação entre os ruídos}

Vamos comparar, agora, o impacto do tipo de ruído no desempenho dos estimadores com integral. Analisando os gráficos 6.13 a 6.27 vemos que, na comparação entre os estimadores, o ruído 1 gera um desempenho pior para os estimadores com integral. Nos gráficos 6.28, 6.29 e 6.30 apresentamos a comparação entre os erros quadráticos, dos estimadores com integral, para os três tipos de ruídos. Nos gráficos 6.28 e 6.29 os percentuais são calculados em relação ao ruído 1. No gráfico 6.28 os percentuais são calculados em relação ao ruído 2. Analisando esses gráficos, vemos que o ruído 1 gera estimativas com erros quadráticos maiores que nos ruídos 2 e 3. Comparando os ruídos 2 e 3 têm desempenhos equivalentes.

Para os gráficos 6.31 a 6.57 somamos os erros quadráticos dos coeficientes de cada equação, obtendo o erro quadrático da equação. Esses gráficos apresentam os erros quadráticos das equações 
em função do número de ruídos. Vemos que, de maneira geral, os erros diminuem rapidamente conforme o número de ruídos aumenta. A análise dos gráficos fica comprometida nos casos em que temos valores extremos para os erros, ou no caso das soluções numéricas onde o erro devido a aproximação tem um impacto grande. Apresentamos apenas os gráficos para os estimadores com integral. 


\begin{tabular}{|l|l|l|l|}
\hline & Todas Soluções & Soluções Analíticas & Soluções Numéricas \\
\hline$t=0,49$ & $1.632864 \mathrm{e}-05$ & $6.396653 \mathrm{e}-07$ & $3.590974 \mathrm{e}-05$ \\
\hline$t=0,24$ & $3.922618 \mathrm{e}-03$ & $1.925182 \mathrm{e}-07$ & $1.200217 \mathrm{e}-03$ \\
\hline$t=0,74$ & $1.14759 \mathrm{e}-04$ & $1.068331 \mathrm{e}-05$ & $2.184561 \mathrm{e}-05$ \\
\hline
\end{tabular}

Tabela 6.2: Média dos erros quadráticos dos estimadores diretos, sem ruído

\begin{tabular}{|ll|l|}
\hline Todas Soluções & Est. Int. & $3.136192 \mathrm{e}-03$ \\
& Est. Dir. & $1.632864 \mathrm{e}-05$ \\
\hline Soluções Analíticas & Est. Int. & $3.054339 \mathrm{e}-07$ \\
& Est. Dir. & $6.396653 \mathrm{e}-07$ \\
\hline Soluções Numéricas & Est. Int. & $5.665937 \mathrm{e}-03$ \\
& Est. Dir. & $3.590974 \mathrm{e}-05$ \\
\hline
\end{tabular}

Tabela 6.3: Média dos erros quadráticos de soluções sem ruído

\begin{tabular}{|ll|l|l|l|}
\hline & & 1 ruído & 100 ruídos & 1000 ruídos \\
\hline Todas Soluções & Est. Int. & $2.344323 \mathrm{e}-01$ & $3.546933 \mathrm{e}-03$ & $3.54976 \mathrm{e}-03$ \\
& Est. Dir. & $\mathbf{1 . 7 0 1 2 8 8 e + 0 0}$ & $2.876142 \mathrm{e}-02$ & $2.162657 \mathrm{e}-03$ \\
\hline Soluções Analíticas & Est. Int. & $6.592539 \mathrm{e}-02$ & $1.519608 \mathrm{e}-04$ & $2.132596 \mathrm{e}-05$ \\
& Est. Dir. & $\mathbf{2 . 0 6 5 6 5 5 e + 0 0}$ & $3.491737 \mathrm{e}-02$ & $2.616959 \mathrm{e}-03$ \\
\hline Soluções Numéricas & Est. Int. & $\mathbf{1 . 0 2 0 7 9 8 e + 0 0}$ & $1.939014 \mathrm{e}-02$ & $2.001578 \mathrm{e}-02$ \\
& Est. Dir. & $9.10991 \mathrm{e}-04$ & $3.362251 \mathrm{e}-05$ & $4.258184 \mathrm{e}-05$ \\
\hline
\end{tabular}

Tabela 6.4: Média dos erros quadráticos de soluções com ruído 1 


\begin{tabular}{|ll|l|l|l|}
\hline & & 1 ruído & 100 ruídos & 1000 ruídos \\
\hline Todas Soluções & Est. Int. & $\mathbf{7 . 2 0 9 4 8 4 e + 0 0}$ & $1.104165 \mathrm{e}-01$ & $1.162946 \mathrm{e}-02$ \\
& Est. Dir. & $\mathbf{7 . 1 9 6 8 3 1 e}+\mathbf{0 0}$ & $4.727842 \mathrm{e}-01$ & $1.388381 \mathrm{e}-01$ \\
\hline Soluções Analíticas & Est. Int. & $\mathbf{8 . 7 4 9 9 2 8 e + 0 0}$ & $3.745326 \mathrm{e}-02$ & $5.851308 \mathrm{e}-03$ \\
& Est. Dir. & $\mathbf{8 . 6 7 0 1 9 3 e + 0 0}$ & $5.731711 \mathrm{e}-01$ & $1.684273 \mathrm{e}-01$ \\
\hline Soluções Numéricas & Est. Int. & $2.074814 \mathrm{e}-02$ & $4.509117 \mathrm{e}-01$ & $3.859416 \mathrm{e}-02$ \\
& Est. Dir. & $3.211422 \mathrm{e}-01$ & $4.312065 \mathrm{e}-03$ & $7.555129 \mathrm{e}-04$ \\
\hline
\end{tabular}

Tabela 6.5: Média dos erros quadráticos de soluções com ruído 2

\begin{tabular}{|ll|l|l|l|}
\hline & & 1 ruído & 100 ruídos & 1000 ruídos \\
\hline Todas Soluções & Est. Int. & $\mathbf{1 . 1 0 5 4 5 e}+\mathbf{0 1}$ & $6.098425 \mathrm{e}-02$ & $6.806416 \mathrm{e}-03$ \\
& Est. Dir. & $\mathbf{1 . 5 7 3 2 0 5 e}+\mathbf{0 1}$ & $2.266108 \mathrm{e}-02$ & $2.628303 \mathrm{e}-02$ \\
\hline Soluções Analíticas & Est. Int. & $\mathbf{1 . 0 9 6 1 4 5 e}+\mathbf{0 1}$ & $3.916334 \mathrm{e}-02$ & $1.988549 \mathrm{e}-03$ \\
& Est. Dir. & $\mathbf{1 . 8 2 9 3 1 e + 0 1}$ & $2.633716 \mathrm{e}-02$ & $3.184197 \mathrm{e}-02$ \\
\hline Soluções Numéricas & Est. Int. & $\mathbf{1 . 1 4 8 8 7 2 e}+\mathbf{0 1}$ & $1.628151 \mathrm{e}-01$ & $2.92898 \mathrm{e}-02$ \\
& Est. Dir. & $\mathbf{3 . 7 8 0 4 7 8 e + 0 0}$ & $5.506064 \mathrm{e}-03$ & $3.412744 \mathrm{e}-04$ \\
\hline
\end{tabular}

Tabela 6.6: Média dos erros quadráticos de soluções com ruído 3

\begin{tabular}{|ll|l|l|l|}
\hline & & 1 ruído & 100 ruídos & 1000 ruídos \\
\hline Todas Soluções & Est. Int. & $1.704275 \mathrm{e}-03$ & $2.449886 \mathrm{e}-04$ & $2.255814 \mathrm{e}-04$ \\
& Est. Dir. & $9.326878 \mathrm{e}-02$ & $6.612153 \mathrm{e}-04$ & $5.803193 \mathrm{e}-05$ \\
\hline Soluções Analíticas & Est. Int. & $1.617154 \mathrm{e}-03$ & $4.250622 \mathrm{e}-06$ & $5.749864 \mathrm{e}-07$ \\
& Est. Dir. & $1.086283 \mathrm{e}-01$ & $7.665495 \mathrm{e}-04$ & $6.521635 \mathrm{e}-05$ \\
\hline Soluções Numéricas & Est. Int. & $2.227002 \mathrm{e}-03$ & $1.689416 \mathrm{e}-03$ & $1.57562 \mathrm{e}-03$ \\
& Est. Dir. & $1.111648 \mathrm{e}-03$ & $2.921039 \mathrm{e}-05$ & $1.492541 \mathrm{e}-05$ \\
\hline
\end{tabular}

Tabela 6.7: Média, sem valores extremos, dos erros quadráticos de soluções com ruído 1

\begin{tabular}{|ll|l|l|l|}
\hline & & 1 ruído & 100 ruídos & 1000 ruídos \\
\hline Todas Soluções & Est. Int. & $2.166023 \mathrm{e}-02$ & $1.23142 \mathrm{e}-01$ & $1.05443 \mathrm{e}-02$ \\
& Est. Dir. & $4.54987 \mathrm{e}-01$ & $5.370253 \mathrm{e}-03$ & $5.077054 \mathrm{e}-04$ \\
\hline Soluções Analíticas & Est. Int. & $2.200226 \mathrm{e}-02$ & $2.283925 \mathrm{e}-04$ & $2.560276 \mathrm{e}-05$ \\
& Est. Dir. & $5.051787 \mathrm{e}-01$ & $5.767073 \mathrm{e}-03$ & $4.147776 \mathrm{e}-04$ \\
\hline Soluções Numéricas & Est. Int. & $2.074814 \mathrm{e}-02$ & $4.509117 \mathrm{e}-01$ & $3.859416 \mathrm{e}-02$ \\
& Est. Dir. & $3.211422 \mathrm{e}-01$ & $4.312065 \mathrm{e}-03$ & $7.555129 \mathrm{e}-04$ \\
\hline
\end{tabular}

Tabela 6.8: Média, sem valores extremos, dos erros quadráticos de soluções com ruído 2

\begin{tabular}{|ll|l|l|l|}
\hline & & 1 ruído & 100 ruídos & 1000 ruídos \\
\hline Todas Soluções & Est. Int. & $2.003838 \mathrm{e}-02$ & $3.384169 \mathrm{e}-04$ & $1.235497 \mathrm{e}-04$ \\
& Est. Dir. & $4.878429 \mathrm{e}-01$ & $6.639505 \mathrm{e}-03$ & $3.746051 \mathrm{e}-04$ \\
\hline Soluções Analíticas & Est. Int. & $2.231371 \mathrm{e}-02$ & $2.713463 \mathrm{e}-04$ & $2.467791 \mathrm{e}-05$ \\
& Est. Dir. & $5.468477 \mathrm{e}-01$ & $7.441494 \mathrm{e}-03$ & $4.166336 \mathrm{e}-04$ \\
\hline Soluções Numéricas & Est. Int. & $1.835723 \mathrm{e}-03$ & $8.749824 \mathrm{e}-04$ & $9.145241 \mathrm{e}-04$ \\
& Est. Dir. & $1.580476 \mathrm{e}-02$ & $2.235935 \mathrm{e}-04$ & $3.837713 \mathrm{e}-05$ \\
\hline
\end{tabular}

Tabela 6.9: Média, sem valores extremos, dos erros quadráticos de soluções com ruído 3 


\begin{tabular}{|c|c|c|c|c|c|}
\hline & & $\mathbf{F}$ & $\mathrm{G}$ & $\mathbf{H}$ & $\mathbf{K}$ \\
\hline \multirow[t]{2}{*}{ Eq. 1} & Int. & 1.44 & 6.925 & $2.959569 \mathrm{e}-13$ & $1.015582 \mathrm{e}-17$ \\
\hline & Est. Dir. & $1.442469 \mathrm{e}-10$ & $6.973277 \mathrm{e}-14$ & $3.00709 \mathrm{e}-13$ & $1.001313 \mathrm{e}-17$ \\
\hline \multirow[t]{2}{*}{ Eq. 2} & Est. Int. & $1.371343 \mathrm{e}-10$ & $1.136387 \mathrm{e}-09$ & $2.671216 \mathrm{e}-09$ & $9.412512 \mathrm{e}-11$ \\
\hline & Est. Dir. & $1.435452 \mathrm{e}-10$ & $9.608113 \mathrm{e}-10$ & $5.363091 \mathrm{e}-10$ & $9.407024 \mathrm{e}-11$ \\
\hline \multirow[t]{2}{*}{ Eq. 3} & Est. Int. & $1.247077 \mathrm{e}-07$ & $1.326063 \mathrm{e}-08$ & $5.196875 \mathrm{e}-08$ & 1.712 \\
\hline & Est. Dir. & $1.414324 \mathrm{e}-06$ & $1.878168 \mathrm{e}-07$ & $2.307641 \mathrm{e}-07$ & 2.005 \\
\hline \multirow[t]{2}{*}{ Eq. 4} & Est. Int. & $2.455941 \mathrm{e}-09$ & $3.120509 \mathrm{e}-23$ & $4.687926 \mathrm{e}-09$ & $3.462827 \mathrm{e}-10$ \\
\hline & Est. Dir. & $9.215143 \mathrm{e}-10$ & $2.166914 \mathrm{e}-23$ & $1.523247 \mathrm{e}-09$ & 1.488 \\
\hline \multirow[t]{2}{*}{ Eq. 5} & Est. Int. & 1.79 & 1.503 & $2.222309 \mathrm{e}-09$ & $1.258386 \mathrm{e}-10$ \\
\hline & Est. Dir. & 9.85 & 8.287 & $1.282333 \mathrm{e}-09$ & 2.46 \\
\hline \multirow[t]{2}{*}{ Eq. 6} & Est. Int. & $9.993079 \mathrm{e}-11$ & $5.059031 \mathrm{e}-24$ & $4.332953 \mathrm{e}-11$ & $5.516225 \mathrm{e}-11$ \\
\hline & Est. Dir. & $7.594965 \mathrm{e}-10$ & $1.629977 \mathrm{e}-23$ & $2.015737 \mathrm{e}-10$ & $4.832306 \mathrm{e}-10$ \\
\hline \multirow[t]{2}{*}{ Eq. 7} & Est. Int. & 2.90 & 2.650 & $1.103407 \mathrm{e}-09$ & 1.454 \\
\hline & Est. Dir. & 5.19 & 5.726 & 2.333 & 5.78 \\
\hline \multirow[t]{2}{*}{ Eq. 8} & Est. Int. & $1.37802 \mathrm{e}-07$ & 5.8978 & $7.316216 \mathrm{e}-07$ & $2.317363 \mathrm{e}-08$ \\
\hline & Est. Dir. & $4.656026 \mathrm{e}-07$ & $2.043093 \mathrm{e}-08$ & $2.224039 \mathrm{e}-06$ & $9.967528 \mathrm{e}-08$ \\
\hline \multirow[t]{2}{*}{ Eq. 9} & Est. Int. & 1.07 & 5.79 & 2.210 & 1.07 \\
\hline & Est. Dir. & 3.92 & 3.208 & $6.625965 \mathrm{e}-11$ & 1.530 \\
\hline \multirow[t]{2}{*}{ Eq. 10a } & Est. Int. & $4.868855 \mathrm{e}-10$ & 1.7673 & $2.438093 \mathrm{e}-09$ & $1.233084 \mathrm{e}-10$ \\
\hline & Est. Dir. & 4.683 & 9.3975 & $1.633557 \mathrm{e}-09$ & $1.298598 \mathrm{e}-10$ \\
\hline \multirow[t]{2}{*}{ Eq. 10b } & Est. Int. & $3.6^{\prime}$ & 2.32 & 3.376 & 4.79 \\
\hline & Est. Dir. & $6.686906 \mathrm{e}-06$ & 1.7347 & $6.259597 \mathrm{e}-07$ & $1.388949 \mathrm{e}-09$ \\
\hline \multirow[t]{2}{*}{ Eq. 11a } & Est. Int. & $8.278491 \mathrm{e}-08$ & $3.189052 \mathrm{e}-09$ & $2.278207 \mathrm{e}-08$ & $5.639017 \mathrm{e}-09$ \\
\hline & Est. Dir. & $9.832268 \mathrm{e}-08$ & $2.883386 \mathrm{e}-09$ & $2.834209 \mathrm{e}-08$ & 7.213762e-09 \\
\hline \multirow[t]{2}{*}{ Eq. 11b } & Est. Int. & $2.423337 \mathrm{e}-07$ & 3.3822 & $2.948874 \mathrm{e}-08$ & 8.662 \\
\hline & Est. Dir. & $2.796292 \mathrm{e}-07$ & 3.0626 & $3.60029 \mathrm{e}-08$ & $1.090815 \mathrm{e}-08$ \\
\hline \multirow[t]{2}{*}{ Eq. 11c } & Est. Int. & $1.352574 \mathrm{e}-09$ & $2.278426 \mathrm{e}-11$ & $6.283996 \mathrm{e}-10$ & $2.185095 \mathrm{e}-10$ \\
\hline & Est. Dir. & $1.517306 \mathrm{e}-09$ & $2.115735 \mathrm{e}-11$ & 7.340823e-10 & $2.72109 \mathrm{e}-10$ \\
\hline \multirow[t]{2}{*}{ Eq. $12 \mathrm{a}$} & Est. Int. & $1.635185 \mathrm{e}-02$ & $3.269863 \mathrm{e}-02$ & $9.6038 \mathrm{e}-04$ & $1.368767 \mathrm{e}-04$ \\
\hline & Est. Dir. & $8.782619 \mathrm{e}-05$ & $1.361403 \mathrm{e}-04$ & $1.181016 \mathrm{e}-05$ & $1.648893 \mathrm{e}-07$ \\
\hline \multirow[t]{2}{*}{ Eq. $12 b$} & Est. Int. & $4.002695 \mathrm{e}-04$ & $3.290389 \mathrm{e}-04$ & $1.867863 \mathrm{e}-04$ & $4.433074 \mathrm{e}-09$ \\
\hline & Est. Dir. & 3.84 & 3.446 & 8.552 & 2.016 \\
\hline \multirow[t]{2}{*}{ Eq. 12c } & Est. Int. & $2.456908 \mathrm{e}-04$ & $1.677367 \mathrm{e}-03$ & $3.228037 \mathrm{e}-04$ & $1.835312 \mathrm{e}-08$ \\
\hline & Est. Dir. & $1.95189 \mathrm{e}-05$ & $2.488938 \mathrm{e}-06$ & $2.327409 \mathrm{e}-06$ & $1.728801 \mathrm{e}-07$ \\
\hline
\end{tabular}

Tabela 6.10: Erros quadráticos para soluções sem ruído 


\begin{tabular}{|c|c|c|c|c|c|}
\hline & & $\mathbf{F}$ & $\mathrm{G}$ & $\mathbf{H}$ & K \\
\hline \multirow{3}{*}{ Eq. 1} & $t=0,49$ & $1.442469 \mathrm{e}-10$ & $6.973277 \mathrm{e}-14$ & $3.00709 \mathrm{e}-13$ & $1.001313 \mathrm{e}-17$ \\
\hline & $t=0,24$ & $1.304042 \mathrm{e}-10$ & $5.143624 \mathrm{e}-13$ & $4.11988 \mathrm{e}-12$ & $1.58574 \mathrm{e}-17$ \\
\hline & $t=0,74$ & $1.427792 \mathrm{e}-10$ & $9.749892 \mathrm{e}-14$ & $3.133859 \mathrm{e}-13$ & $1.145708 \mathrm{e}-17$ \\
\hline \multirow[t]{3}{*}{ Eq. 2} & $t=0,49$ & $1.435452 \mathrm{e}-10$ & $9.608113 \mathrm{e}-10$ & $5.363091 \mathrm{e}-10$ & $9.407024 \mathrm{e}-11$ \\
\hline & $t=0,24$ & $1.445104 \mathrm{e}-10$ & $9.93444 \mathrm{e}-10$ & $5.775544 \mathrm{e}-10$ & $9.385046 \mathrm{e}-11$ \\
\hline & $t=0,74$ & $1.432772 \mathrm{e}-10$ & $9.364672 \mathrm{e}-10$ & $5.121432 \mathrm{e}-10$ & $9.548602 \mathrm{e}-11$ \\
\hline \multirow[t]{3}{*}{ Eq. 3} & $t=0,49$ & $1.414324 \mathrm{e}-06$ & $1.878168 \mathrm{e}-07$ & $2.307641 \mathrm{e}-07$ & $2.005797 \mathrm{e}-12$ \\
\hline & $t=0,24$ & $4.620282 \mathrm{e}-08$ & $1.336969 \mathrm{e}-07$ & $1.629963 \mathrm{e}-07$ & $1.747036 \mathrm{e}-14$ \\
\hline & $t=0,74$ & $1.370135 \mathrm{e}-04$ & $3.224667 \mathrm{e}-06$ & $1.496548 \mathrm{e}-03$ & $1.321172 \mathrm{e}-06$ \\
\hline \multirow[t]{3}{*}{ Eq. 4} & $t=0,49$ & $9.215143 \mathrm{e}-10$ & $2.166914 \mathrm{e}-23$ & $1.523247 \mathrm{e}-09$ & $1.488103 \mathrm{e}-13$ \\
\hline & $t=0,24$ & $9.215144 \mathrm{e}-10$ & $1.203539 \mathrm{e}-23$ & $1.523247 \mathrm{e}-09$ & $1.488107 \mathrm{e}-13$ \\
\hline & $t=0,74$ & $9.215144 \mathrm{e}-10$ & $1.265836 \mathrm{e}-23$ & $1.523247 \mathrm{e}-09$ & $1.488102 \mathrm{e}-13$ \\
\hline \multirow[t]{3}{*}{ Eq. 5} & $t=0,49$ & $9.855229 \mathrm{e}-10$ & $8.287248 \mathrm{e}-10$ & $1.282333 \mathrm{e}-09$ & $2.466531 \mathrm{e}-11$ \\
\hline & $t=0,24$ & $6.915574 \mathrm{e}-10$ & $6.101787 \mathrm{e}-10$ & $8.769349 \mathrm{e}-10$ & $2.666996 \mathrm{e}-13$ \\
\hline & $t=0,74$ & $1.197654 \mathrm{e}-09$ & $1.035893 \mathrm{e}-09$ & $1.633484 \mathrm{e}-09$ & $6.279847 \mathrm{e}-11$ \\
\hline \multirow[t]{3}{*}{ Eq. 6} & $t=0,49$ & $7.594965 \mathrm{e}-10$ & $1.629977 \mathrm{e}-23$ & $2.015737 \mathrm{e}-10$ & $4.832306 \mathrm{e}-10$ \\
\hline & $t=0,24$ & $5.815339 \mathrm{e}-10$ & $9 \mathrm{e}-23$ & $71 \mathrm{e}-08$ & $5.1868 \mathrm{e}-10$ \\
\hline & $t=0,74$ & $1.563977 \mathrm{e}-09$ & $1.944451 \mathrm{e}-23$ & $1.020025 \mathrm{e}-10$ & $7.960039 \mathrm{e}-10$ \\
\hline \multirow[t]{3}{*}{ Eq. 7} & $t=0,49$ & $5.198132 \mathrm{e}-09$ & $5.726117 \mathrm{e}-10$ & $2.333552 \mathrm{e}-09$ & $5.785614 \mathrm{e}-10$ \\
\hline & $t=0,24$ & $4.438495 \mathrm{e}-09$ & $3.096931 \mathrm{e}-10$ & $2.156182 \mathrm{e}-09$ & $5.671003 \mathrm{e}-10$ \\
\hline & $t=0,74$ & $6.031057 \mathrm{e}-09$ & 8.991 & $5 \mathrm{e}-09$ & $5.642697 \mathrm{e}-10$ \\
\hline \multirow[t]{3}{*}{ Eq. 8} & $t=0,49$ & $4.656026 \mathrm{e}-07$ & $2.043093 \mathrm{e}-08$ & $2.224039 \mathrm{e}-06$ & $9.967528 \mathrm{e}-08$ \\
\hline & $t=0,24$ & $2.589295 \mathrm{e}-07$ & $1.797017 \mathrm{e}-08$ & $9.794534 \mathrm{e}-07$ & $5.229341 \mathrm{e}-08$ \\
\hline & $t=0,74$ & $1.114747 \mathrm{e}-05$ & $5.657105 \mathrm{e}-07$ & $2.780557 \mathrm{e}-05$ & $7.156865 \mathrm{e}-08$ \\
\hline \multirow[t]{3}{*}{ Eq. 9} & $t=0,49$ & $3.920274 \mathrm{e}-10$ & $3.208931 \mathrm{e}-10$ & $6.625965 \mathrm{e}-11$ & $1.530576 \mathrm{e}-12$ \\
\hline & $t=0,24$ & $3.719799 \mathrm{e}-10$ & $62 \mathrm{e}-10$ & $5.68566 \mathrm{e}-11$ & $1.527965 \mathrm{e}-12$ \\
\hline & $t=0,74$ & $4.191759 \mathrm{e}-10$ & $3.696299 \mathrm{e}-10$ & $7.718292 \mathrm{e}-11$ & $1.509343 \mathrm{e}-12$ \\
\hline \multirow[t]{3}{*}{ Eq. 10a } & $t=0,49$ & $4.683117 \mathrm{e}-10$ & $9.397518 \mathrm{e}-10$ & $1.633557 \mathrm{e}-09$ & $1.298598 \mathrm{e}-10$ \\
\hline & $t=0,24$ & 5.41099 & 4.016 & $3 e-11$ & 1.62 \\
\hline & $t=0,74$ & $9.509992 \mathrm{e}-10$ & $1.864093 \mathrm{e}-09$ & $1.876668 \mathrm{e}-09$ & $1.364235 \mathrm{e}-10$ \\
\hline \multirow[t]{3}{*}{ Eq. 10b } & $t=0,49$ & $6.686906 \mathrm{e}-06$ & $1.734732 \mathrm{e}-09$ & $6.259597 \mathrm{e}-07$ & $1.388949 \mathrm{e}-09$ \\
\hline & $t=0,24$ & $1.146029 \mathrm{e}-06$ & $6.643331 \mathrm{e}-12$ & $4.028396 \mathrm{e}-09$ & $8.772556 \mathrm{e}-10$ \\
\hline & $t=0,74$ & $1.215637 \mathrm{e}-06$ & $1.815595 \mathrm{e}-09$ & 7.477248e-09 & $9.449437 \mathrm{e}-11$ \\
\hline \multirow[t]{3}{*}{ Eq. 11a } & $t=0,49$ & $9.832268 \mathrm{e}-08$ & $2.883386 \mathrm{e}-09$ & $2.834209 \mathrm{e}-08$ & $7.213762 \mathrm{e}-09$ \\
\hline & $t=0,24$ & $3.778804 \mathrm{e}-07$ & $7.938022 \mathrm{e}-09$ & $2.87704 \mathrm{e}-07$ & $4.468593 \mathrm{e}-08$ \\
\hline & $t=0,74$ & $4.143906 \mathrm{e}-08$ & $1.789383 \mathrm{e}-09$ & $9.750964 \mathrm{e}-09$ & $2.132585 \mathrm{e}-09$ \\
\hline \multirow[t]{3}{*}{ Eq. 11b } & $t=0,49$ & $2.796292 \mathrm{e}-07$ & $3.062648 \mathrm{e}-09$ & $3.60029 \mathrm{e}-08$ & $1.090815 \mathrm{e}-08$ \\
\hline & $t=0,24$ & $8.548688 \mathrm{e}-07$ & 8.02819e-09 & $3.073755 \mathrm{e}-07$ & $5.90987 \mathrm{e}-08$ \\
\hline & $t=0,74$ & $1.361837 \mathrm{e}-07$ & $2.073277 \mathrm{e}-09$ & $1.442952 \mathrm{e}-08$ & $3.464712 \mathrm{e}-09$ \\
\hline \multirow[t]{3}{*}{ Eq. 11c } & $t=0,49$ & $1.517306 \mathrm{e}-09$ & $2.115735 \mathrm{e}-11$ & $7.340823 \mathrm{e}-10$ & $2.72109 \mathrm{e}-10$ \\
\hline & $t=0,24$ & $3.523022 \mathrm{e}-09$ & $1.234285 \mathrm{e}-11$ & $2.222066 \mathrm{e}-09$ & $1.159574 \mathrm{e}-09$ \\
\hline & $t=0,74$ & $7.904417 \mathrm{e}-10$ & $2.978037 \mathrm{e}-11$ & $3.42075 \mathrm{e}-10$ & $8.881447 \mathrm{e}-11$ \\
\hline \multirow[t]{3}{*}{ Eq. $12 \mathrm{a}$} & $t=0,49$ & $8.782619 \mathrm{e}-05$ & $1.361403 \mathrm{e}-04$ & $1.181016 \mathrm{e}-05$ & $1.648893 \mathrm{e}-07$ \\
\hline & $t=0,24$ & $5.791834 \mathrm{e}-04$ & $1.980856 \mathrm{e}-03$ & $3.937893 \mathrm{e}-05$ & $2.529553 \mathrm{e}-05$ \\
\hline & $t=0,74$ & $1.776687 \mathrm{e}-05$ & $3.570721 \mathrm{e}-05$ & $6.907389 \mathrm{e}-06$ & $5.168706 \mathrm{e}-08$ \\
\hline \multirow[t]{3}{*}{ Eq. $12 \mathrm{~b}$} & $t=0,49$ & $3.841451 \mathrm{e}-07$ & $3.446669 \mathrm{e}-06$ & $8.55246 \mathrm{e}-07$ & $2.016346 \mathrm{e}-09$ \\
\hline & $t=0,24$ & $5.553811 \mathrm{e}-07$ & $6.153626 \mathrm{e}-06$ & $1.098012 \mathrm{e}-05$ & $1.447241 \mathrm{e}-09$ \\
\hline & $t=0,74$ & $8.306814 \mathrm{e}-06$ & $2.878912 \mathrm{e}-06$ & $1.413089 \mathrm{e}-05$ & $1.186166 \mathrm{e}-08$ \\
\hline \multirow[t]{3}{*}{ Eq. 12c } & $t=0,49$ & $1.95189 \mathrm{e}-05$ & $2.488938 \mathrm{e}-06$ & $2.327409 \mathrm{e}-06$ & $1.728801 \mathrm{e}-07$ \\
\hline & $t=0,24$ & $3.020912 \mathrm{e}-03$ & $5.562439 \mathrm{e}-02$ & $4.704692 \mathrm{e}-03$ & $6.872516 \mathrm{e}-04$ \\
\hline & $t=0,74$ & $3.946315 \mathrm{e}-05$ & $1.211577 \mathrm{e}-04$ & $2.462092 \mathrm{e}-05$ & $7.371386 \mathrm{e}-07$ \\
\hline
\end{tabular}

Tabela 6.11: Erros quadráticos para soluções sem ruído, estimadores diretos 


\begin{tabular}{|c|c|c|c|c|c|}
\hline & & $\mathbf{F}$ & $\mathrm{G}$ & $\mathbf{H}$ & $\mathbf{K}$ \\
\hline \multirow[t]{2}{*}{ Eq. 1} & Est. Int. & $3.252415 \mathrm{e}-04$ & $1.218095 \mathrm{e}-03$ & $1.186063 \mathrm{e}-03$ & $4.771167 \mathrm{e}-09$ \\
\hline & Est. Dir. & $1.704654 \mathrm{e}-03$ & $4.947287 \mathrm{e}-03$ & $1.041576 \mathrm{e}-02$ & $7.860428 \mathrm{e}-07$ \\
\hline \multirow{2}{*}{ Eq. 2} & Est. Int. & $2.985366 \mathrm{e}-06$ & $2.778027 \mathrm{e}-04$ & $2.99717 \mathrm{e}-03$ & $1.711128 \mathrm{e}-08$ \\
\hline & Est. Dir. & $1.212279 \mathrm{e}-04$ & $3.204585 \mathrm{e}-04$ & $9.064712 \mathrm{e}-03$ & $5.927246 \mathrm{e}-07$ \\
\hline \multirow[t]{2}{*}{ Eq. 3} & Est. Int. & $7.73637 \mathrm{e}-04$ & $9.033563 \mathrm{e}-05$ & $2.261881 \mathrm{e}-04$ & $9.245535 \mathrm{e}-09$ \\
\hline & Est. Dir. & $1.037306 \mathrm{e}+01$ & $1.50857 \mathrm{e}+00$ & $1.147369 \mathrm{e}+00$ & $6.408726 \mathrm{e}-03$ \\
\hline \multirow[t]{2}{*}{ Eq. 4} & Est. Int. & $1.48836 \mathrm{e}-05$ & $2.560004 \mathrm{e}-04$ & $9.008642 \mathrm{e}-05$ & $1.237819 \mathrm{e}-09$ \\
\hline & Est. Dir. & $5.890843 \mathrm{e}-06$ & $5.852283 \mathrm{e}-05$ & $2.7771 \mathrm{e}-05$ & $7.827849 \mathrm{e}-10$ \\
\hline \multirow[t]{2}{*}{ Eq. 5} & Est. Int. & $4.424556 \mathrm{e}-04$ & $3.581978 \mathrm{e}-03$ & $1.466542 \mathrm{e}-04$ & $2.273636 \mathrm{e}-09$ \\
\hline & Est. Dir. & $1.316168 \mathrm{e}-04$ & $7.14375 \mathrm{e}-04$ & $3.347828 \mathrm{e}-05$ & $1.206365 \mathrm{e}-08$ \\
\hline \multirow[t]{2}{*}{ Eq. 6} & Est. Int. & $1.955148 \mathrm{e}-04$ & $1.126044 \mathrm{e}-04$ & $1.317997 \mathrm{e}-03$ & $6.572039 \mathrm{e}-07$ \\
\hline & Est. Dir. & $3.317785 \mathrm{e}-02$ & $3.177173 \mathrm{e}-03$ & $3.982581 \mathrm{e}-01$ & $9.634459 \mathrm{e}-04$ \\
\hline \multirow[t]{2}{*}{ Eq. 7} & Est. Int. & $1.038557 \mathrm{e}-03$ & $1.069044 \mathrm{e}-03$ & $5.74606 \mathrm{e}-04$ & $8.24986 \mathrm{e}-08$ \\
\hline & Est. Dir. & $1.409194 \mathrm{e}-01$ & $1.410733 \mathrm{e}-01$ & $6.206319 \mathrm{e}-02$ & $9.603504 \mathrm{e}-04$ \\
\hline \multirow[t]{2}{*}{ Eq. 8} & Est. Int. & $6.509871 \mathrm{e}-05$ & $3.064082 \mathrm{e}-04$ & $6.307165 \mathrm{e}-04$ & $1.989607 \mathrm{e}-07$ \\
\hline & Est. Dir. & $3.85896 \mathrm{e}-03$ & $1.186933 \mathrm{e}-02$ & $1.232074 \mathrm{e}-02$ & $1.406783 \mathrm{e}-06$ \\
\hline \multirow[t]{2}{*}{ Eq. 9} & Est. Int. & $1.256049 \mathrm{e}-04$ & $4.387463 \mathrm{e}-04$ & $6.099774 \mathrm{e}-04$ & $7.648942 \mathrm{e}-09$ \\
\hline & Est. Dir. & $2.918022 \mathrm{e}-02$ & $5.517309 \mathrm{e}-02$ & $1.821886 \mathrm{e}-02$ & $1.156159 \mathrm{e}-06$ \\
\hline \multirow[t]{2}{*}{ Eq. 10a } & Est. Int. & $7.941727 \mathrm{e}-05$ & $1.337061 \mathrm{e}-04$ & $4.299663 \mathrm{e}-04$ & $2.259879 \mathrm{e}-09$ \\
\hline & Est. Dir. & $5.795501 \mathrm{e}-03$ & $4.199739 \mathrm{e}-03$ & $5.316336 \mathrm{e}-03$ & $1.459953 \mathrm{e}-06$ \\
\hline \multirow[t]{2}{*}{ Eq. 10b } & Est. Int. & $8.239256 \mathrm{e}-01$ & $1.589641 \mathrm{e}-03$ & $7.63484 \mathrm{e}-02$ & $5.958238 \mathrm{e}-04$ \\
\hline & Est. Dir. & $1.328838 \mathrm{e}+01$ & $1.70496 \mathrm{e}-02$ & $1.273197 \mathrm{e}+00$ & $1.589554 \mathrm{e}-03$ \\
\hline \multirow[t]{2}{*}{ Eq. 11a } & Est. Int. & $9.730941 \mathrm{e}-05$ & $1.972237 \mathrm{e}-04$ & $2.70103 \mathrm{e}-04$ & $1.724324 \mathrm{e}-07$ \\
\hline & Est. Dir. & $2.104159 \mathrm{e}-02$ & $3.354536 \mathrm{e}-02$ & $7.069504 \mathrm{e}-02$ & $8.296458 \mathrm{e}-05$ \\
\hline \multirow[t]{2}{*}{ Eq. 11b } & Est. Int. & $1.05179 \mathrm{e}-04$ & $1.955183 \mathrm{e}-04$ & $2.680394 \mathrm{e}-04$ & $1.840573 \mathrm{e}-07$ \\
\hline & Est. Dir. & $2.731685 \mathrm{e}-02$ & $3.481039 \mathrm{e}-02$ & $7.233836 \mathrm{e}-02$ & $9.091001 \mathrm{e}-05$ \\
\hline \multirow[t]{2}{*}{ Eq. 11c } & Est. Int. & $6.67503 \mathrm{e}-05$ & $2.304755 \mathrm{e}-04$ & $3.064128 \mathrm{e}-04$ & $1.222236 \mathrm{e}-07$ \\
\hline & Est. Dir. & $5.533077 \mathrm{e}-03$ & $2.648284 \mathrm{e}-02$ & $5.748307 \mathrm{e}-02$ & $4.24705 \mathrm{e}-05$ \\
\hline \multirow[t]{2}{*}{ Eq. $12 \mathrm{a}$} & Est. Int. & $1.015667 \mathrm{e}+00$ & $1.978538 \mathrm{e}+00$ & $5.549503 \mathrm{e}-02$ & $8.238986 \mathrm{e}-03$ \\
\hline & Est. Dir. & $1.725752 \mathrm{e}-04$ & $2.915995 \mathrm{e}-04$ & $4.51863 \mathrm{e}-05$ & $3.159109 \mathrm{e}-07$ \\
\hline \multirow[t]{2}{*}{ Eq. $12 b$} & Est. Int. & $6.900301 \mathrm{e}-05$ & $2.182314 \mathrm{e}-04$ & $1.442984 \mathrm{e}-04$ & $1.192645 \mathrm{e}-08$ \\
\hline & Est. Dir. & $3.805164 \mathrm{e}-06$ & $3.651405 \mathrm{e}-05$ & $2.752632 \mathrm{e}-05$ & $2.022812 \mathrm{e}-09$ \\
\hline \multirow[t]{2}{*}{ Eq. 12c } & Est. Int. & $3.567518 \mathrm{e}-04$ & $3.172244 \mathrm{e}-03$ & $4.932203 \mathrm{e}-04$ & $2.434923 \mathrm{e}-07$ \\
\hline & Est. Dir. & $3.674799 \mathrm{e}-04$ & $1.464212 \mathrm{e}-03$ & $3.098959 \mathrm{e}-04$ & $1.386088 \mathrm{e}-05$ \\
\hline
\end{tabular}

Tabela 6.12: Erros quadráticos para soluções com ruído 1, 1 ruído 


\begin{tabular}{|c|c|c|c|c|c|}
\hline & & $\mathbf{F}$ & $\mathrm{G}$ & $\mathbf{H}$ & $\mathbf{K}$ \\
\hline \multirow[t]{2}{*}{ Eq. 1} & Est. Int. & $3.209541 \mathrm{e}-07$ & $1.920523 \mathrm{e}-06$ & $2.558734 \mathrm{e}-06$ & $3.305818 \mathrm{e}-11$ \\
\hline & Est. Dir. & $7.112734 \mathrm{e}-06$ & $2.656265 \mathrm{e}-05$ & $4.140805 \mathrm{e}-05$ & $3.513443 \mathrm{e}-09$ \\
\hline \multirow[t]{2}{*}{ Eq. 2} & Est. Int. & $9.517581 \mathrm{e}-09$ & $3.332975 \mathrm{e}-07$ & $4.071057 \mathrm{e}-06$ & $1.37725 \mathrm{e}-10$ \\
\hline & Est. Dir. & $4.317184 \mathrm{e}-07$ & $2.9049 \mathrm{e}-06$ & $4.752965 \mathrm{e}-05$ & $1.91104 \mathrm{e}-09$ \\
\hline \multirow[t]{2}{*}{ Eq. 3} & Est. Int. & $1.12191 \mathrm{e}-06$ & $4.108996 \mathrm{e}-07$ & $5.462348 \mathrm{e}-07$ & $1.708592 \mathrm{e}-09$ \\
\hline & Est. Dir. & $4.12431 \mathrm{e}-05$ & $5.752779 \mathrm{e}-06$ & $6.23983 \mathrm{e}-06$ & $5.198548 \mathrm{e}-09$ \\
\hline \multirow[t]{2}{*}{ Eq. 4} & Est. Int. & $91 \mathrm{e}-08$ & $2.400424 \mathrm{e}-07$ & $5.717104 \mathrm{e}-08$ & $4.149523 \mathrm{e}-10$ \\
\hline & Est. Dir. & $9.173564 \mathrm{e}-08$ & 1.170 & $6.610228 \mathrm{e}-07$ & 1.854 \\
\hline \multirow[t]{2}{*}{ Eq. 5} & Est. Int. & $1.469578 \mathrm{e}-06$ & $5.08055 \mathrm{e}-06$ & $1.190051 \mathrm{e}-07$ & $97 \mathrm{e}-10$ \\
\hline & Est. Dir. & $2.122144 \mathrm{e}-06$ & $9.318719 \mathrm{e}-06$ & $4.747816 \mathrm{e}-07$ & $9.994832 \mathrm{e}-11$ \\
\hline \multirow[t]{2}{*}{ Eq. 6} & Est. Int. & 1.21 & 1.324 & $6.018705 \mathrm{e}-06$ & $1.864385 \mathrm{e}-09$ \\
\hline & Est. Dir. & 1.64 & 8.573 & $1.289054 \mathrm{e}-03$ & 9.56 \\
\hline \multirow{2}{*}{ Eq. 7} & Est. Int. & $4.08097 \mathrm{e}-06$ & $2.846571 \mathrm{e}-06$ & $1.759367 \mathrm{e}-06$ & $1.99076 \mathrm{e}-09$ \\
\hline & Est. Dir. & $3.913362 \mathrm{e}-04$ & $9.391879 \mathrm{e}-04$ & $2.377401 \mathrm{e}-04$ & $1.159981 \mathrm{e}-07$ \\
\hline \multirow[t]{2}{*}{ Eq. 8} & Est. Int. & 5.82 & $4.57^{\circ}$ & $2.166654 \mathrm{e}-06$ & 2.32 \\
\hline & Est. Dir. & 7.43 & 1.02 & 2.156 & 8.42 \\
\hline \multirow[t]{2}{*}{ Eq. 9} & Est. Int. & $1.342541 \mathrm{e}-06$ & $1.38712 \mathrm{e}-06$ & $6.565212 \mathrm{e}-07$ & $3.150531 \mathrm{e}-11$ \\
\hline & Est. Dir. & 1.59 & 4.462 & $2.301433 \mathrm{e}-04$ & $2.074364 \mathrm{e}-08$ \\
\hline \multirow[t]{2}{*}{ Eq. 10a } & Est. Int. & 2.95 & 2.75 & $2.849422 \mathrm{e}-07$ & 1.628 \\
\hline & Est. Dir. & 2.726 & 5.2536 & $5.879563 \mathrm{e}-05$ & 3e-09 \\
\hline \multirow[t]{2}{*}{ Eq. 10b } & Est. Int. & $1.893638 \mathrm{e}-03$ & $3.401044 \mathrm{e}-06$ & $1.768426 \mathrm{e}-04$ & $4.810628 \mathrm{e}-07$ \\
\hline & Est. Dir. & $4.375962 \mathrm{e}-01$ & $7.227 !$ & $4.097514 \mathrm{e}-02$ & $2.972551 \mathrm{e}-04$ \\
\hline \multirow[t]{2}{*}{ Eq. 11a } & Est. Int. & 1.41 & 1.142 & 1.229 & $7.175191 \mathrm{e}-09$ \\
\hline & Est. Dir. & $2 \mathrm{e}-04$ & $8.909445 \mathrm{e}-05$ & $1.053577 \mathrm{e}-03$ & $4.243513 \mathrm{e}-07$ \\
\hline \multirow[t]{2}{*}{ Eq. 11b } & Est. Int. & $1.74085 \mathrm{e}-06$ & $1.135278 \mathrm{e}-06$ & $1.070704 \mathrm{e}-06$ & $1.04234 \mathrm{e}-08$ \\
\hline & Est. Dir. & $7.395106 \mathrm{e}-04$ & $9.64623 \mathrm{e}-05$ & $1.093007 \mathrm{e}-03$ & $4.311461 \mathrm{e}-07$ \\
\hline \multirow[t]{2}{*}{ Eq. 11c } & Est. Int. & $9.302361 \mathrm{e}-07$ & $1.262401 \mathrm{e}-06$ & $1.285437 \mathrm{e}-06$ & $3.20376 \mathrm{e}-10$ \\
\hline & Est. Dir. & $1.404005 \mathrm{e}-04$ & $5.93615 \mathrm{e}-05$ & $8.087255 \mathrm{e}-04$ & $4.076421 \mathrm{e}-07$ \\
\hline \multirow[t]{2}{*}{ Eq. $12 \mathrm{a}$} & Est. Int. & $1.788687 \mathrm{e}-02$ & $3.571893 \mathrm{e}-02$ & $1.041868 \mathrm{e}-03$ & $1.43906 \mathrm{e}-04$ \\
\hline & Est. Dir. & $1.231843 \mathrm{e}-05$ & $2.674325 \mathrm{e}-05$ & $3.219316 \mathrm{e}-06$ & $1.657336 \mathrm{e}-07$ \\
\hline \multirow{2}{*}{ Eq. $12 b$} & Est. Int. & $4.110883 \mathrm{e}-04$ & $3.453413 \mathrm{e}-04$ & $1.996633 \mathrm{e}-04$ & $4.687133 \mathrm{e}-09$ \\
\hline & Est. Dir. & $4.221741 \mathrm{e}-07$ & $4.339177 \mathrm{e}-06$ & $1.435652 \mathrm{e}-06$ & $1.744939 \mathrm{e}-09$ \\
\hline \multirow[t]{2}{*}{ Eq. 12c } & Est. Int. & $2.641578 \mathrm{e}-04$ & $1.824056 \mathrm{e}-03$ & $3.344992 \mathrm{e}-04$ & $2.27585 \mathrm{e}-08$ \\
\hline & Est. Dir. & $2.225931 \mathrm{e}-05$ & $2.424303 \mathrm{e}-05$ & $5.022776 \mathrm{e}-06$ & $6.969187 \mathrm{e}-07$ \\
\hline
\end{tabular}

Tabela 6.13: Erros quadráticos para soluções com ruído 1, 100 ruídos 


\begin{tabular}{|c|c|c|c|c|c|}
\hline & & $\mathbf{F}$ & $\mathrm{G}$ & $\mathbf{H}$ & $\mathbf{K}$ \\
\hline \multirow[t]{2}{*}{ Eq. 1} & Int. & 3.38 & $1.032131 \mathrm{e}-07$ & 1.84 & 1.04 \\
\hline & Est. Dir. & $1.193063 \mathrm{e}-06$ & $3.996392 \mathrm{e}-06$ & $6.280071 \mathrm{e}-06$ & $8.566882 \mathrm{e}-10$ \\
\hline \multirow[t]{2}{*}{ Eq. 2} & Est. Int. & $1.352377 \mathrm{e}-09$ & $3.250022 \mathrm{e}-08$ & $3.963313 \mathrm{e}-07$ & $1.980627 \mathrm{e}-10$ \\
\hline & Est. Dir. & $8.997185 \mathrm{e}-08$ & $3.791409 \mathrm{e}-07$ & $5.223991 \mathrm{e}-06$ & $2.112163 \mathrm{e}-09$ \\
\hline \multirow[t]{2}{*}{ Eq. 3} & Est. Int. & $2.165762 \mathrm{e}-07$ & $3.171978 \mathrm{e}-08$ & $1.056875 \mathrm{e}-07$ & $1.984519 \mathrm{e}-09$ \\
\hline & Est. Dir. & 3.433 & 5.2627 & 5.883 & $2.142707 \mathrm{e}-10$ \\
\hline \multirow[t]{2}{*}{ Eq. 4} & Est. Int. & $9.662693 \mathrm{e}-09$ & $3.375544 \mathrm{e}-08$ & $1.655827 \mathrm{e}-08$ & $3.698795 \mathrm{e}-10$ \\
\hline & Est. Dir. & $1.155596 \mathrm{e}-08$ & $9.649052 \mathrm{e}-08$ & $3.007423 \mathrm{e}-08$ & $6.616457 \mathrm{e}-13$ \\
\hline \multirow[t]{2}{*}{ Eq. 5} & Est. Int. & 1.028 & 4.8450 & $1.449203 \mathrm{e}-08$ & $1.078291 \mathrm{e}-10$ \\
\hline & Est. Dir. & 2.86 & 1.2356 & 3.144 & $62 \mathrm{e}-11$ \\
\hline \multirow[t]{2}{*}{ Eq. 6} & Est. Int. & 4.645 & $1.383922 \mathrm{e}-08$ & $2.491669 \mathrm{e}-07$ & $4.580227 \mathrm{e}-10$ \\
\hline & Est. Dir. & 1.355 & 2.8202 & $1.862399 \mathrm{e}-04$ & $5.726328 \mathrm{e}-08$ \\
\hline \multirow[t]{2}{*}{ Eq. 7} & Est. Int. & 2.30 & 3.368 & $1.516072 \mathrm{e}-07$ & $282 \mathrm{e}-11$ \\
\hline & Est. Dir. & 4.28 & 7.656 & 5.455 & e-08 \\
\hline \multirow[t]{2}{*}{ Eq. 8} & Est. Int. & 2.49 & 9.8904 & $9.836487 \mathrm{e}-07$ & $2.909584 \mathrm{e}-08$ \\
\hline & Est. Dir. & 6.370 & 6.3299 & $4.799573 \mathrm{e}-05$ & $1.791377 \mathrm{e}-07$ \\
\hline \multirow[t]{2}{*}{ Eq. 9} & Est. Int. & 6.142 & $\overline{1.48}$ & 9.597 & $8.351979 \mathrm{e}-12$ \\
\hline & Est. Dir. & 7.514 & 2.370 & 3.1922 & $83 e-10$ \\
\hline \multirow[t]{2}{*}{ Eq. 10a } & Est. Int. & 2.882 & 7.33 & $1.182076 \mathrm{e}-07$ & $2.565775 \mathrm{e}-10$ \\
\hline & Est. Dir. & $2.44 \S$ & 3.865 & $6.539747 \mathrm{e}-06$ & $1.091011 \mathrm{e}-09$ \\
\hline \multirow[t]{2}{*}{ Eq. 10b } & Est. Int. & 2.663 & 5.160 & 2.423 & $9 \mathrm{e}-07$ \\
\hline & Est. Dir. & 3.271 & 5.6035 & 3.0614 & $1.704014 \mathrm{e}-05$ \\
\hline \multirow[t]{2}{*}{ Eq. 11a } & Est. Int. & $2.485608 \mathrm{e}-07$ & 7.0524 & $5.085627 \mathrm{e}-07$ & $6.759077 \mathrm{e}-09$ \\
\hline & Est. Dir. & 3.366 & 1.901 & $3.992327 \mathrm{e}-05$ & $2.123982 \mathrm{e}-08$ \\
\hline \multirow[t]{2}{*}{ Eq. 11b } & Est. Int. & 4.115 & 7.0691 & $5.140589 \mathrm{e}-07$ & $1.002746 \mathrm{e}-08$ \\
\hline & Est. Dir. & 4.089 & 1.9365 & $4.260606 \mathrm{e}-05$ & $2.607735 \mathrm{e}-08$ \\
\hline \multirow[t]{2}{*}{ Eq. 11c } & Est. Int. & $1.021042 \mathrm{e}-07$ & $6.641533 \mathrm{e}-08$ & $5.596025 \mathrm{e}-07$ & $9.227323 \mathrm{e}-11$ \\
\hline & & 1.1579 & $1.549826 \mathrm{e}-05$ & $2.760644 \mathrm{e}-05$ & $5.072831 \mathrm{e}-09$ \\
\hline \multirow[t]{2}{*}{ Eq. $12 \mathrm{a}$} & Est. Int. & $1.85986 \mathrm{e}-02$ & $3.705942 \mathrm{e}-02$ & $1.081628 \mathrm{e}-03$ & $1.564665 \mathrm{e}-04$ \\
\hline & Est. Dir. & $3.426452 \mathrm{e}-05$ & $5.787949 \mathrm{e}-05$ & $5.591905 \mathrm{e}-06$ & $1.587958 \mathrm{e}-07$ \\
\hline \multirow[t]{2}{*}{ Eq. $12 b$} & Est. Int. & $4.076116 \mathrm{e}-04$ & $3.442586 \mathrm{e}-04$ & $1.97583 \mathrm{e}-04$ & $4.573322 \mathrm{e}-09$ \\
\hline & & $1.789601 \mathrm{e}-07$ & 3.55050 & $9.009902 \mathrm{e}-07$ & $1.960846 \mathrm{e}-09$ \\
\hline \multirow[t]{2}{*}{ Eq. 12c } & Est. Int. & $2.427219 \mathrm{e}-04$ & $1.640232 \mathrm{e}-03$ & $3.187985 \mathrm{e}-04$ & $3.038924 \mathrm{e}-08$ \\
\hline & Est. Dir. & $1.874438 \mathrm{e}-05$ & $3.986297 \mathrm{e}-06$ & $2.412684 \mathrm{e}-06$ & $7.504456 \mathrm{e}-08$ \\
\hline
\end{tabular}

Tabela 6.14: Erros quadráticos para soluções com ruído 1, 1000 ruídos 


\begin{tabular}{|c|c|c|c|c|c|}
\hline & & $\mathbf{F}$ & $\mathrm{G}$ & $\mathbf{H}$ & $\mathbf{K}$ \\
\hline \multirow{2}{*}{ Eq. 1} & Est. Int. & $3.104908 \mathrm{e}-03$ & $7.837265 \mathrm{e}-03$ & $9.14135 \mathrm{e}-03$ & $4.401432 \mathrm{e}-08$ \\
\hline & Est. Dir. & $1.254277 \mathrm{e}-02$ & $4.565636 \mathrm{e}-02$ & $5.994281 \mathrm{e}-02$ & $1.167726 \mathrm{e}-05$ \\
\hline \multirow[t]{2}{*}{ Eq. 2} & Est. Int. & $8.211881 \mathrm{e}-05$ & $2.269228 \mathrm{e}-03$ & $3.532586 \mathrm{e}-02$ & $9.753896 \mathrm{e}-08$ \\
\hline & Est. Dir. & $7.195234 \mathrm{e}-04$ & $4.88301 \mathrm{e}-03$ & $5.913612 \mathrm{e}-02$ & $1.569682 \mathrm{e}-06$ \\
\hline \multirow[t]{2}{*}{ Eq. 3} & Est. Int. & $5.679162 \mathrm{e}-03$ & $1.786619 \mathrm{e}-03$ & $3.02375 \mathrm{e}-03$ & $6.880693 \mathrm{e}-08$ \\
\hline & Est. Dir. & $8.503972 \mathrm{e}-01$ & $8.946143 \mathrm{e}-02$ & $6.525529 \mathrm{e}-02$ & $1.468858 \mathrm{e}-04$ \\
\hline \multirow[t]{2}{*}{ Eq. 4} & Est. Int. & $3.299612 \mathrm{e}-04$ & $2.683869 \mathrm{e}-03$ & $9.841947 \mathrm{e}-04$ & $7.160027 \mathrm{e}-09$ \\
\hline & Est. Dir. & $3.109258 \mathrm{e}-03$ & $3.134041 \mathrm{e}-02$ & $1.166642 \mathrm{e}-02$ & $2.444805 \mathrm{e}-06$ \\
\hline \multirow[t]{2}{*}{ Eq. 5} & Est. Int. & $7.584444 \mathrm{e}-03$ & $3.993652 \mathrm{e}-02$ & $1.530673 \mathrm{e}-03$ & $1.049947 \mathrm{e}-07$ \\
\hline & Est. Dir. & $7.848868 \mathrm{e}-02$ & $4.185568 \mathrm{e}-01$ & $1.75822 \mathrm{e}-02$ & $9.020869 \mathrm{e}-06$ \\
\hline \multirow[t]{2}{*}{ Eq. 6} & Est. Int. & $4.578484 \mathrm{e}-03$ & $1.252629 \mathrm{e}-03$ & $7.178593 \mathrm{e}-02$ & $1.060892 \mathrm{e}-06$ \\
\hline & Est. Dir. & $3.30451 \mathrm{e}-01$ & $2.705159 \mathrm{e}-02$ & $3.731615 \mathrm{e}+00$ & $1.457413 \mathrm{e}-02$ \\
\hline \multirow[t]{2}{*}{ Eq. 7} & Est. Int. & $1.654071 \mathrm{e}-02$ & $3.022439 \mathrm{e}-02$ & $1.232391 \mathrm{e}-02$ & $4.989822 \mathrm{e}-07$ \\
\hline & Est. Dir. & $3.477024 \mathrm{e}+00$ & $1.767296 \mathrm{e}+00$ & $8.556896 \mathrm{e}-01$ & $1.137376 \mathrm{e}-02$ \\
\hline \multirow[t]{2}{*}{ Eq. 8} & Est. Int. & $2.893012 \mathrm{e}-03$ & $4.335236 \mathrm{e}-03$ & $1.036132 \mathrm{e}-02$ & $4.330257 \mathrm{e}-06$ \\
\hline & Est. Dir. & $1.36329 \mathrm{e}-01$ & $2.151155 \mathrm{e}-01$ & $3.530587 \mathrm{e}-01$ & $8.260958 \mathrm{e}-03$ \\
\hline \multirow[t]{2}{*}{ Eq. 9} & Est. Int. & $7.02328 \mathrm{e}-03$ & $1.337945 \mathrm{e}-02$ & $8.169597 \mathrm{e}-03$ & $3.080046 \mathrm{e}-07$ \\
\hline & Est. Dir. & $3.175774 \mathrm{e}-01$ & $7.135249 \mathrm{e}-01$ & $2.896719 \mathrm{e}-01$ & $1.470455 \mathrm{e}-04$ \\
\hline \multirow[t]{2}{*}{ Eq. 10a } & Est. Int. & $2.480845 \mathrm{e}-03$ & $2.492035 \mathrm{e}-03$ & $3.578363 \mathrm{e}-03$ & $8.352608 \mathrm{e}-08$ \\
\hline & Est. Dir. & $2.792243 \mathrm{e}-02$ & $1.217009 \mathrm{e}-01$ & $1.091279 \mathrm{e}-01$ & $8.333511 \mathrm{e}-05$ \\
\hline \multirow[t]{2}{*}{ Eq. 10b } & Est. Int. & $1.11612 \mathrm{e}+02$ & $2.091163 \mathrm{e}-01$ & $1.02036 \mathrm{e}+01$ & $5.816203 \mathrm{e}-02$ \\
\hline & Est. Dir. & $2.874646 \mathrm{e}+01$ & $6.833497 \mathrm{e}-02$ & $3.398033 \mathrm{e}+00$ & $2.236683 \mathrm{e}-02$ \\
\hline \multirow[t]{2}{*}{ Eq. 11a } & Est. Int. & $6.750883 \mathrm{e}-03$ & $1.057047 \mathrm{e}-02$ & $1.708201 \mathrm{e}-02$ & $4.385205 \mathrm{e}-06$ \\
\hline & Est. Dir. & $1.442329 \mathrm{e}+01$ & $3.433858 \mathrm{e}-01$ & $3.574695 \mathrm{e}+00$ & $1.875998 \mathrm{e}-01$ \\
\hline \multirow[t]{2}{*}{ Eq. 11b } & Est. Int. & $7.232635 \mathrm{e}-03$ & $1.053904 \mathrm{e}-02$ & $1.700001 \mathrm{e}-02$ & $4.439477 \mathrm{e}-06$ \\
\hline & Est. Dir. & $4.773499 \mathrm{e}+01$ & $8.250606 \mathrm{e}-01$ & $5.771309 \mathrm{e}+00$ & $3.234087 \mathrm{e}-01$ \\
\hline \multirow[t]{2}{*}{ Eq. 11c } & Est. Int. & 4.330373e-03 & $1.130962 \mathrm{e}-02$ & $1.860462 \mathrm{e}-02$ & 4.11393e-06 \\
\hline & Est. Dir. & $4.237399 \mathrm{e}-01$ & $9.751597 \mathrm{e}-02$ & $1.159078 \mathrm{e}+00$ & $2.693455 \mathrm{e}-02$ \\
\hline \multirow[t]{2}{*}{ Eq. 12a } & Est. Int. & $1.105938 \mathrm{e}-02$ & $2.558733 \mathrm{e}-02$ & $8.585449 \mathrm{e}-04$ & $4.953085 \mathrm{e}-05$ \\
\hline & Est. Dir. & $9.256692 \mathrm{e}-02$ & $2.062142 \mathrm{e}-01$ & $1.398515 \mathrm{e}-02$ & $5.046984 \mathrm{e}-04$ \\
\hline \multirow[t]{2}{*}{ Eq. $12 b$} & Est. Int. & $5.272993 \mathrm{e}-04$ & $5.872117 \mathrm{e}-04$ & $5.287384 \mathrm{e}-04$ & $1.223716 \mathrm{e}-08$ \\
\hline & Est. Dir. & $2.380063 \mathrm{e}-03$ & $1.0424 \mathrm{e}-02$ & $9.220683 \mathrm{e}-03$ & $1.925061 \mathrm{e}-05$ \\
\hline \multirow[t]{2}{*}{ Eq. 12c } & Est. Int. & $1.440938 \mathrm{e}-03$ & $1.925017 \mathrm{e}-02$ & $2.343285 \mathrm{e}-03$ & $1.200206 \mathrm{e}-05$ \\
\hline & Est. Dir. & $1.109938 \mathrm{e}-01$ & $4.317553 \mathrm{e}-01$ & $8.481367 \mathrm{e}-02$ & $5.489694 \mathrm{e}-04$ \\
\hline
\end{tabular}

Tabela 6.15: Erros quadráticos para soluções com ruído 2, 1 ruído 


\begin{tabular}{|c|c|c|c|c|c|}
\hline & & $\mathbf{F}$ & $\mathrm{G}$ & $\mathbf{H}$ & $\mathbf{K}$ \\
\hline \multirow[t]{2}{*}{ Eq. 1} & Est. Int. & $2.22644 \mathrm{e}-05$ & $8.782093 \mathrm{e}-05$ & $9.026994 \mathrm{e}-05$ & $4.531056 \mathrm{e}-10$ \\
\hline & Est. Dir. & $3.932925 \mathrm{e}-04$ & $1.683758 \mathrm{e}-03$ & $1.849835 \mathrm{e}-03$ & $4.80202 \mathrm{e}-09$ \\
\hline \multirow[t]{2}{*}{ Eq. 2} & Est. Int. & $1.075451 \mathrm{e}-06$ & $2.358682 \mathrm{e}-05$ & $2.878408 \mathrm{e}-04$ & $3.001031 \mathrm{e}-09$ \\
\hline & Est. Dir. & $9.871044 \mathrm{e}-06$ & $1.030902 \mathrm{e}-04$ & $1.657822 \mathrm{e}-03$ & $2.209822 \mathrm{e}-08$ \\
\hline \multirow[t]{2}{*}{ Eq. 3} & Est. Int. & $3.453095 \mathrm{e}-05$ & $1.823412 \mathrm{e}-05$ & $4.146822 \mathrm{e}-05$ & $1.360793 \mathrm{e}-09$ \\
\hline & Est. Dir. & $1.251544 \mathrm{e}-02$ & $1.891116 \mathrm{e}-03$ & $1.917714 \mathrm{e}-03$ & $4.591752 \mathrm{e}-06$ \\
\hline \multirow[t]{2}{*}{ Eq. 4} & Est. Int. & $2.494708 \mathrm{e}-06$ & $3.75136 \mathrm{e}-05$ & $1.642864 \mathrm{e}-05$ & $6.357535 \mathrm{e}-10$ \\
\hline & Est. Dir. & $3.085853 \mathrm{e}-05$ & $2.446903 \mathrm{e}-04$ & $9.775545 \mathrm{e}-05$ & $2.667774 \mathrm{e}-08$ \\
\hline \multirow[t]{2}{*}{ Eq. 5} & Est. Int. & $5.865383 \mathrm{e}-05$ & $3.88953 \mathrm{e}-04$ & $1.647115 \mathrm{e}-05$ & $5.08134 \mathrm{e}-09$ \\
\hline & Est. Dir. & $6.794899 \mathrm{e}-04$ & $3.135078 \mathrm{e}-03$ & $1.268222 \mathrm{e}-04$ & $3.052916 \mathrm{e}-07$ \\
\hline \multirow[t]{2}{*}{ Eq. 6} & Est. Int. & $5.802573 \mathrm{e}-05$ & $1.276149 \mathrm{e}-05$ & $5.585536 \mathrm{e}-04$ & $8.954513 \mathrm{e}-09$ \\
\hline & Est. Dir. & $4.511315 \mathrm{e}-03$ & $3.594963 \mathrm{e}-04$ & $5.921339 \mathrm{e}-02$ & $8.576758 \mathrm{e}-04$ \\
\hline \multirow[t]{2}{*}{ Eq. 7} & Est. Int. & $1.495344 \mathrm{e}-04$ & $3.684759 \mathrm{e}-04$ & $1.246806 \mathrm{e}-04$ & $2.067375 \mathrm{e}-08$ \\
\hline & Est. Dir. & $6.389127 \mathrm{e}-03$ & $1.098048 \mathrm{e}-02$ & $3.856849 \mathrm{e}-03$ & $2.556224 \mathrm{e}-05$ \\
\hline \multirow[t]{2}{*}{ Eq. 8} & Est. Int. & $2.877879 \mathrm{e}-05$ & $6.270823 \mathrm{e}-05$ & $1.494748 \mathrm{e}-04$ & $6.443689 \mathrm{e}-08$ \\
\hline & Est. Dir. & $9.558897 \mathrm{e}-04$ & $2.27921 \mathrm{e}-03$ & $3.567482 \mathrm{e}-03$ & $1.212913 \mathrm{e}-06$ \\
\hline \multirow[t]{2}{*}{ Eq. 9} & Est. Int. & $6.963689 \mathrm{e}-05$ & $1.847864 \mathrm{e}-04$ & $9.476134 \mathrm{e}-05$ & $5.863034 \mathrm{e}-09$ \\
\hline & Est. Dir. & $1.941303 \mathrm{e}-03$ & $5.063339 \mathrm{e}-03$ & $3.074005 \mathrm{e}-03$ & $4.900767 \mathrm{e}-07$ \\
\hline \multirow[t]{2}{*}{ Eq. 10a } & Est. Int. & $1.659581 \mathrm{e}-05$ & $3.643458 \mathrm{e}-05$ & $5.62742 \mathrm{e}-05$ & $1.546283 \mathrm{e}-09$ \\
\hline & Est. Dir. & $4.139221 \mathrm{e}-04$ & $1.111136 \mathrm{e}-03$ & $1.386355 \mathrm{e}-03$ & $6.592477 \mathrm{e}-07$ \\
\hline \multirow[t]{2}{*}{ Eq. $10 b$} & Est. Int. & $4.751422 \mathrm{e}-01$ & $8.796274 \mathrm{e}-04$ & $4.377115 \mathrm{e}-02$ & $2.03388 \mathrm{e}-04$ \\
\hline & Est. Dir. & $7.116909 \mathrm{e}+00$ & $1.314001 \mathrm{e}-02$ & $6.986868 \mathrm{e}-01$ & $2.64004 \mathrm{e}-04$ \\
\hline \multirow[t]{2}{*}{ Eq. 11a } & Est. Int. & $4.927586 \mathrm{e}-05$ & $8.337258 \mathrm{e}-05$ & $2.835009 \mathrm{e}-04$ & $1.127631 \mathrm{e}-07$ \\
\hline & Est. Dir. & $7.191514 \mathrm{e}-03$ & $2.099716 \mathrm{e}-03$ & $1.370181 \mathrm{e}-02$ & $3.399881 \mathrm{e}-05$ \\
\hline \multirow[t]{2}{*}{ Eq. 11b } & Est. Int. & $5.456002 \mathrm{e}-05$ & $8.3122 \mathrm{e}-05$ & $2.830105 \mathrm{e}-04$ & $1.229688 \mathrm{e}-07$ \\
\hline & Est. Dir. & $9.929503 \mathrm{e}-03$ & $2.203656 \mathrm{e}-03$ & $1.418089 \mathrm{e}-02$ & $3.581478 \mathrm{e}-05$ \\
\hline \multirow[t]{2}{*}{ Eq. 11c } & Est. Int. & $2.866054 \mathrm{e}-05$ & $8.899328 \mathrm{e}-05$ & $2.952803 \mathrm{e}-04$ & $6.64889 \mathrm{e}-08$ \\
\hline & Est. Dir. & $1.636393 \mathrm{e}-03$ & $1.528442 \mathrm{e}-03$ & $1.049828 \mathrm{e}-02$ & $2.417846 \mathrm{e}-05$ \\
\hline \multirow[t]{2}{*}{ Eq. $12 \mathrm{a}$} & Est. Int. & $4.475822 \mathrm{e}-01$ & $8.728553 \mathrm{e}-01$ & $2.425027 \mathrm{e}-02$ & $3.717519 \mathrm{e}-03$ \\
\hline & Est. Dir. & $3.698273 \mathrm{e}-04$ & $8.632843 \mathrm{e}-04$ & $1.103103 \mathrm{e}-04$ & $3.231322 \mathrm{e}-06$ \\
\hline \multirow[t]{2}{*}{ Eq. $12 b$} & Est. Int. & $4.213197 \mathrm{e}-04$ & $3.395142 \mathrm{e}-04$ & $1.988148 \mathrm{e}-04$ & $4.400914 \mathrm{e}-09$ \\
\hline & Est. Dir. & $3.571367 \mathrm{e}-05$ & $1.042378 \mathrm{e}-04$ & $9.035492 \mathrm{e}-05$ & $3.578776 \mathrm{e}-08$ \\
\hline \multirow[t]{2}{*}{ Eq. 12c } & Est. Int. & $3.408595 \mathrm{e}-04$ & $2.634377 \mathrm{e}-03$ & $3.945635 \mathrm{e}-04$ & $3.85681 \mathrm{e}-07$ \\
\hline & Est. Dir. & $2.247279 \mathrm{e}-03$ & $8.003676 \mathrm{e}-03$ & $9.189836 \mathrm{e}-04$ & $1.892625 \mathrm{e}-04$ \\
\hline
\end{tabular}

Tabela 6.16: Erros quadráticos para soluções com ruído 2, 100 ruídos 


\begin{tabular}{|c|c|c|c|c|c|}
\hline & & $\mathbf{F}$ & $\mathrm{G}$ & $\mathbf{H}$ & $\mathbf{K}$ \\
\hline \multirow[t]{2}{*}{ Eq. 1} & Est. Int. & $3.673162 \mathrm{e}-06$ & $1.007477 \mathrm{e}-05$ & $8.169974 \mathrm{e}-06$ & $5.719709 \mathrm{e}-10$ \\
\hline & Est. Dir. & $2.837105 \mathrm{e}-05$ & $7.782281 \mathrm{e}-05$ & $9.033858 \mathrm{e}-05$ & $5.591548 \mathrm{e}-09$ \\
\hline \multirow[t]{2}{*}{ Eq. 2} & Est. Int. & $1.022577 \mathrm{e}-07$ & $3.417093 \mathrm{e}-06$ & $3.929063 \mathrm{e}-05$ & $8.836665 \mathrm{e}-10$ \\
\hline & Est. Dir. & $1.504899 \mathrm{e}-06$ & $7.691516 \mathrm{e}-06$ & $9.018761 \mathrm{e}-05$ & $1.555714 \mathrm{e}-08$ \\
\hline \multirow[t]{2}{*}{ Eq. 3} & Est. Int. & $6.277704 \mathrm{e}-06$ & $1.64274 \mathrm{e}-06$ & $4.346126 \mathrm{e}-06$ & $2.140346 \mathrm{e}-09$ \\
\hline & Est. Dir. & $9.390489 \mathrm{e}-04$ & $1.244562 \mathrm{e}-04$ & $1.207299 \mathrm{e}-04$ & $1.877452 \mathrm{e}-07$ \\
\hline \multirow[t]{2}{*}{ Eq. 4} & Est. Int. & $3.461319 \mathrm{e}-07$ & $4.143191 \mathrm{e}-06$ & $1.69298 \mathrm{e}-06$ & $5.075907 \mathrm{e}-10$ \\
\hline & Est. Dir. & $2.814205 \mathrm{e}-06$ & $1.88765 \mathrm{e}-05$ & $8.263333 \mathrm{e}-06$ & $2.674512 \mathrm{e}-09$ \\
\hline \multirow[t]{2}{*}{ Eq. 5} & Est. Int. & $8.209906 \mathrm{e}-06$ & $4.896772 \mathrm{e}-05$ & $2.138774 \mathrm{e}-06$ & $8.433651 \mathrm{e}-10$ \\
\hline & Est. Dir. & $6.023789 \mathrm{e}-05$ & $2.680189 \mathrm{e}-04$ & $1.349676 \mathrm{e}-05$ & $4.402615 \mathrm{e}-09$ \\
\hline \multirow[t]{2}{*}{ Eq. 6} & Est. Int. & $4.846533 \mathrm{e}-06$ & $1.619469 \mathrm{e}-06$ & $5.525589 \mathrm{e}-05$ & $4.990825 \mathrm{e}-09$ \\
\hline & Est. Dir. & $1.926744 \mathrm{e}-04$ & $2.996828 \mathrm{e}-05$ & $4.385433 \mathrm{e}-03$ & $1.379341 \mathrm{e}-05$ \\
\hline \multirow[t]{2}{*}{ Eq. 7} & Est. Int. & $1.098669 \mathrm{e}-05$ & $2.16566 \mathrm{e}-05$ & $1.228973 \mathrm{e}-05$ & $3.842574 \mathrm{e}-09$ \\
\hline & Est. Dir. & $4.29343 \mathrm{e}-04$ & $7.150666 \mathrm{e}-04$ & $3.102442 \mathrm{e}-04$ & $8.9204 \mathrm{e}-07$ \\
\hline \multirow[t]{2}{*}{ Eq. 8} & Est. Int. & $3.118304 \mathrm{e}-06$ & $7.505177 \mathrm{e}-06$ & $1.319786 \mathrm{e}-05$ & $9.1762 \mathrm{e}-09$ \\
\hline & Est. Dir. & 8.381897e-05 & $1.669726 \mathrm{e}-04$ & $3.088473 \mathrm{e}-04$ & $5.009235 \mathrm{e}-07$ \\
\hline \multirow[t]{2}{*}{ Eq. 9} & Est. Int. & $5.54873 \mathrm{e}-06$ & $1.225268 \mathrm{e}-05$ & $8.706671 \mathrm{e}-06$ & $1.471657 \mathrm{e}-10$ \\
\hline & Est. Dir. & $1.42743 \mathrm{e}-04$ & $3.247543 \mathrm{e}-04$ & $2.318492 \mathrm{e}-04$ & $2.519851 \mathrm{e}-07$ \\
\hline \multirow[t]{2}{*}{ Eq. 10a } & Est. Int. & $1.861418 \mathrm{e}-06$ & $3.403239 \mathrm{e}-06$ & $6.720398 \mathrm{e}-06$ & $1.416706 \mathrm{e}-10$ \\
\hline & Est. Dir. & $4.136742 \mathrm{e}-05$ & $6.548856 \mathrm{e}-05$ & $9.947648 \mathrm{e}-05$ & $7.514893 \mathrm{e}-08$ \\
\hline \multirow[t]{2}{*}{ Eq. 10b } & Est. Int. & $7.445781 \mathrm{e}-02$ & $1.423789 \mathrm{e}-04$ & $6.860662 \mathrm{e}-03$ & $3.48791 \mathrm{e}-05$ \\
\hline & Est. Dir. & $2.138545 \mathrm{e}+0$ & $3.65678 \mathrm{e}-03$ & $1.999016 \mathrm{e}-01$ & $7.469122 \mathrm{e}-04$ \\
\hline \multirow[t]{2}{*}{ Eq. 11a } & Est. Int. & $9.727473 \mathrm{e}-06$ & $9.304793 \mathrm{e}-06$ & $1.808617 \mathrm{e}-05$ & $8.832156 \mathrm{e}-09$ \\
\hline & Est. Dir. & $7.380797 \mathrm{e}-04$ & $1.943128 \mathrm{e}-04$ & $1.15182 \mathrm{e}-03$ & $4.980653 \mathrm{e}-06$ \\
\hline \multirow[t]{2}{*}{ Eq. 11b } & Est. Int. & $1.048546 \mathrm{e}-05$ & $9.275572 \mathrm{e}-06$ & $1.798697 \mathrm{e}-05$ & $1.24086 \mathrm{e}-08$ \\
\hline & Est. Dir. & $1.02603 \mathrm{e}-03$ & $2.002719 \mathrm{e}-04$ & $1.201358 \mathrm{e}-03$ & $5.210185 \mathrm{e}-06$ \\
\hline \multirow[t]{2}{*}{ Eq. 11c } & Est. Int. & $6.195814 \mathrm{e}-06$ & $9.959453 \mathrm{e}-06$ & $2.00413 \mathrm{e}-05$ & $3.843614 \mathrm{e}-10$ \\
\hline & Est. Dir. & $1.668255 \mathrm{e}-04$ & $1.607372 \mathrm{e}-04$ & 8.838938e-04 & $2.549757 \mathrm{e}-06$ \\
\hline \multirow[t]{2}{*}{ Eq. 12a } & Est. Int. & $3.698648 \mathrm{e}-02$ & $7.298215 \mathrm{e}-02$ & $2.075243 \mathrm{e}-03$ & $2.939045 \mathrm{e}-04$ \\
\hline & Est. Dir. & $5.033833 \mathrm{e}-05$ & $1.071563 \mathrm{e}-04$ & $1.175754 \mathrm{e}-05$ & $1.978935 \mathrm{e}-07$ \\
\hline \multirow[t]{2}{*}{ Eq. $12 b$} & Est. Int. & $4.108164 \mathrm{e}-04$ & $3.478325 \mathrm{e}-04$ & $2.006351 \mathrm{e}-04$ & $4.511791 \mathrm{e}-09$ \\
\hline & Est. Dir. & $2.72612 \mathrm{e}-06$ & $1.519382 \mathrm{e}-05$ & $1.065247 \mathrm{e}-05$ & 7.249872e-09 \\
\hline \multirow[t]{2}{*}{ Eq. 12c } & Est. Int. & $2.605676 \mathrm{e}-04$ & $1.88263 \mathrm{e}-03$ & $3.422006 \mathrm{e}-04$ & $1.478474 \mathrm{e}-08$ \\
\hline & Est. Dir. & $4.652428 \mathrm{e}-04$ & $1.483281 \mathrm{e}-03$ & $9.991346 \mathrm{e}-05$ & $2.007134 \mathrm{e}-05$ \\
\hline
\end{tabular}

Tabela 6.17: Erros quadráticos para soluções com ruído 2, 1000 ruídos 


\begin{tabular}{|c|c|c|c|c|c|}
\hline & & $\mathbf{F}$ & $\mathrm{G}$ & $\mathbf{H}$ & $\mathbf{K}$ \\
\hline \multirow[t]{2}{*}{ Eq. 1} & Est. Int. & $3.165953 \mathrm{e}-03$ & $1.237051 \mathrm{e}-02$ & $1.93717 \mathrm{e}-02$ & $5.760834 \mathrm{e}-08$ \\
\hline & Est. Dir. & $1.817124 \mathrm{e}-02$ & $9.964071 \mathrm{e}-02$ & $1.304853 \mathrm{e}-01$ & $3.62312 \mathrm{e}-06$ \\
\hline \multirow[t]{2}{*}{ Eq. 2} & Est. Int. & $4.42382 \mathrm{e}-05$ & $2.197593 \mathrm{e}-03$ & $3.462579 \mathrm{e}-02$ & $1.406106 \mathrm{e}-07$ \\
\hline & Est. Dir. & $6.607856 \mathrm{e}-04$ & $8.893975 \mathrm{e}-03$ & $1.011349 \mathrm{e}-01$ & $3.397724 \mathrm{e}-06$ \\
\hline \multirow[t]{2}{*}{ Eq. 3} & Est. Int. & $8.142244 \mathrm{e}-03$ & $1.626948 \mathrm{e}-03$ & $3.629354 \mathrm{e}-03$ & $4.228861 \mathrm{e}-08$ \\
\hline & Est. Dir. & $9.079466 \mathrm{e}-01$ & $1.719632 \mathrm{e}-01$ & $1.610768 \mathrm{e}-01$ & $7.991989 \mathrm{e}-04$ \\
\hline \multirow[t]{2}{*}{ Eq. 4} & Est. Int. & $4.13719 \mathrm{e}-04$ & $2.209803 \mathrm{e}-03$ & $6.041809 \mathrm{e}-04$ & $1.498577 \mathrm{e}-08$ \\
\hline & Est. Dir. & $2.67309 \mathrm{e}-03$ & $2.461212 \mathrm{e}-02$ & $8.281655 \mathrm{e}-03$ & $3.854596 \mathrm{e}-05$ \\
\hline \multirow{2}{*}{ Eq. 5} & Est. Int. & $8.34397 \mathrm{e}-03$ & $3.822641 \mathrm{e}-02$ & $9.396647 \mathrm{e}-04$ & $1.309275 \mathrm{e}-07$ \\
\hline & Est. Dir. & $1.045765 \mathrm{e}-01$ & $5.000588 \mathrm{e}-01$ & $1.117916 \mathrm{e}-02$ & $4.887363 \mathrm{e}-04$ \\
\hline \multirow[t]{2}{*}{ Eq. 6} & Est. Int. & $4.860679 \mathrm{e}-03$ & $1.019462 \mathrm{e}-03$ & $2.929607 \mathrm{e}-02$ & $2.258838 \mathrm{e}-06$ \\
\hline & Est. Dir. & $4.565354 \mathrm{e}-01$ & $3.344062 \mathrm{e}-02$ & $4.535768 \mathrm{e}+00$ & $2.136781 \mathrm{e}-02$ \\
\hline \multirow[t]{2}{*}{ Eq. 7} & Est. Int. & $2.683748 \mathrm{e}-02$ & $1.982415 \mathrm{e}-02$ & $1.352326 \mathrm{e}-02$ & $1.417672 \mathrm{e}-06$ \\
\hline & Est. Dir. & $3.137209 \mathrm{e}+01$ & $5.609156 \mathrm{e}+00$ & $4.949222 \mathrm{e}+00$ & $6.864108 \mathrm{e}-02$ \\
\hline \multirow[t]{2}{*}{ Eq. 8} & Est. Int. & $3.293302 \mathrm{e}-03$ & $2.873999 \mathrm{e}-03$ & $1.21334 \mathrm{e}-02$ & $1.932013 \mathrm{e}-07$ \\
\hline & Est. Dir. & $6.984028 \mathrm{e}-02$ & $2.008603 \mathrm{e}-01$ & $3.462618 \mathrm{e}-01$ & $6.494884 \mathrm{e}-04$ \\
\hline \multirow[t]{2}{*}{ Eq. 9} & Est. Int. & $4.117311 \mathrm{e}-03$ & $6.887734 \mathrm{e}-03$ & $6.050711 \mathrm{e}-03$ & $3.920641 \mathrm{e}-08$ \\
\hline & Est. Dir. & $4.080305 \mathrm{e}-01$ & $5.631522 \mathrm{e}-01$ & $3.184764 \mathrm{e}-01$ & $2.089863 \mathrm{e}-04$ \\
\hline \multirow[t]{2}{*}{ Eq. 10a } & Est. Int. & $2.746654 \mathrm{e}-03$ & $1.204877 \mathrm{e}-03$ & $3.28895 \mathrm{e}-03$ & $2.65981 \mathrm{e}-08$ \\
\hline & Est. Dir. & $2.8453 \mathrm{e}-02$ & $8.582475 \mathrm{e}-02$ & $1.002557 \mathrm{e}-01$ & $7.979503 \mathrm{e}-05$ \\
\hline \multirow[t]{2}{*}{ Eq. 10b } & Est. Int. & $1.399417 \mathrm{e}+02$ & $2.628772 \mathrm{e}-01$ & $1.283194 \mathrm{e}+01$ & $6.23874 \mathrm{e}-02$ \\
\hline & Est. Dir. & $1.692623 \mathrm{e}+02$ & $7.7512 \mathrm{e}-01$ & $1.398867 \mathrm{e}+01$ & $1.336992 \mathrm{e}-01$ \\
\hline \multirow[t]{2}{*}{ Eq. 11a } & Est. Int. & $1.041393 \mathrm{e}-02$ & $5.305571 \mathrm{e}-03$ & $1.373031 \mathrm{e}-02$ & $1.209789 \mathrm{e}-07$ \\
\hline & Est. Dir. & $4.737849 \mathrm{e}+00$ & $2.843573 \mathrm{e}-01$ & $1.886787 \mathrm{e}+00$ & $1.203438 \mathrm{e}-01$ \\
\hline \multirow[t]{2}{*}{ Eq. 11b } & Est. Int. & $1.117105 \mathrm{e}-02$ & $5.27833 \mathrm{e}-03$ & $1.360607 \mathrm{e}-02$ & $1.211723 \mathrm{e}-07$ \\
\hline & Est. Dir. & $9.211976 \mathrm{e}+00$ & $3.817301 \mathrm{e}-01$ & $2.160591 \mathrm{e}+00$ & $1.473673 \mathrm{e}-01$ \\
\hline \multirow[t]{2}{*}{ Eq. 11c } & Est. Int. & $6.505629 \mathrm{e}-03$ & $5.912075 \mathrm{e}-03$ & $1.569327 \mathrm{e}-02$ & $2.423239 \mathrm{e}-07$ \\
\hline & Est. Dir. & $3.187057 \mathrm{e}-01$ & $1.321487 \mathrm{e}-01$ & $1.105454 \mathrm{e}+00$ & $3.523766 \mathrm{e}-02$ \\
\hline \multirow[t]{2}{*}{ Eq. 12a } & Est. Int. & $1.145173 \mathrm{e}+01$ & $2.226971 \mathrm{e}+01$ & $6.193212 \mathrm{e}-01$ & $9.174685 \mathrm{e}-02$ \\
\hline & Est. Dir. & $1.237599 \mathrm{e}-01$ & $2.510144 \mathrm{e}-01$ & $1.63081 \mathrm{e}-02$ & $3.206703 \mathrm{e}-03$ \\
\hline \multirow[t]{2}{*}{ Eq. 12b } & Est. Int. & $5.396294 \mathrm{e}-04$ & $7.541001 \mathrm{e}-04$ & $5.419151 \mathrm{e}-04$ & 7.892975e-08 \\
\hline & Est. Dir. & $2.687939 \mathrm{e}-03$ & $7.582514 \mathrm{e}-03$ & $5.52172 \mathrm{e}-03$ & $1.258315 \mathrm{e}-05$ \\
\hline \multirow[t]{2}{*}{ Eq. 12c } & Est. Int. & $2.48226 \mathrm{e}-03$ & $2.660445 \mathrm{e}-02$ & $2.73821 \mathrm{e}-03$ & $6.646679 \mathrm{e}-07$ \\
\hline & Est. Dir. & $3.074072 \mathrm{e}+00$ & $7.579761 \mathrm{e}+00$ & $1.985826 \mathrm{e}-01$ & $7.892585 \mathrm{e}-02$ \\
\hline
\end{tabular}

Tabela 6.18: Erros quadráticos para soluções com ruído 3, 1 ruído 


\begin{tabular}{|c|c|c|c|c|c|}
\hline & & $\mathbf{F}$ & $\mathrm{G}$ & $\mathbf{H}$ & K \\
\hline \multirow[t]{2}{*}{ Eq. 1} & Est. Int. & $2.658688 \mathrm{e}-05$ & $1.001103 \mathrm{e}-04$ & $1.211264 \mathrm{e}-04$ & $1.546753 \mathrm{e}-08$ \\
\hline & Est. Dir. & $1.663893 \mathrm{e}-04$ & $5.886837 \mathrm{e}-04$ & $1.01265 \mathrm{e}-03$ & $1.634433 \mathrm{e}-08$ \\
\hline \multirow[t]{2}{*}{ Eq. 2} & Est. Int. & $1.106708 \mathrm{e}-06$ & $1.923514 \mathrm{e}-05$ & $2.68337 \mathrm{e}-04$ & $5.407597 \mathrm{e}-08$ \\
\hline & Est. Dir. & $7.562842 \mathrm{e}-06$ & $7.278452 \mathrm{e}-05$ & $6.594193 \mathrm{e}-04$ & $1.327528 \mathrm{e}-08$ \\
\hline \multirow[t]{2}{*}{ Eq. 3} & Est. Int. & $6.115926 \mathrm{e}-05$ & $2.294891 \mathrm{e}-05$ & $4.658961 \mathrm{e}-05$ & $4.383094 \mathrm{e}-09$ \\
\hline & Est. Dir. & $2.84133 \mathrm{e}-02$ & $4.103528 \mathrm{e}-03$ & $4.529902 \mathrm{e}-03$ & $1.287798 \mathrm{e}-05$ \\
\hline \multirow[t]{2}{*}{ Eq. 4} & Est. Int. & 3.586 & $4.513359 \mathrm{e}-05$ & $1.739879 \mathrm{e}-05$ & $4.067699 \mathrm{e}-09$ \\
\hline & Est. Dir. & 3.359 & $3.426116 \mathrm{e}-04$ & $1.200013 \mathrm{e}-04$ & $7.48279 \mathrm{e}-08$ \\
\hline \multirow[t]{2}{*}{ Eq. 5} & Est. Int. & 8.2251 & $4.908272 \mathrm{e}-04$ & $1.738538 \mathrm{e}-05$ & $1.553644 \mathrm{e}-08$ \\
\hline & Est. Dir. & $5.70231 \mathrm{e}-04$ & $3.478097 \mathrm{e}-03$ & $1.160611 \mathrm{e}-04$ & $3.446677 \mathrm{e}-07$ \\
\hline \multirow[t]{2}{*}{ Eq. 6} & Est. Int. & 7.026 & 1.31 & $5.000697 \mathrm{e}-04$ & $5.176518 \mathrm{e}-07$ \\
\hline & Est. Dir. & 1.806 & 2.217 & $2.230867 \mathrm{e}-02$ & $1.713011 \mathrm{e}-05$ \\
\hline \multirow[t]{2}{*}{ Eq. 7} & Est. Int. & $1.75489 \mathrm{e}-04$ & $4.389033 \mathrm{e}-04$ & $1.824244 \mathrm{e}-04$ & $1.246697 \mathrm{e}-07$ \\
\hline & Est. Dir. & $4.953972 \mathrm{e}-03$ & $7.838599 \mathrm{e}-03$ & $3.410936 \mathrm{e}-03$ & $3.679625 \mathrm{e}-06$ \\
\hline \multirow[t]{2}{*}{ Eq. 8} & Est. Int. & 3.572 & 6.44 & $2.068222 \mathrm{e}-04$ & $1.086527 \mathrm{e}-08$ \\
\hline & Est. Dir. & 6.38 & 1.63 & 3.7068 & 1.94 \\
\hline \multirow[t]{2}{*}{ Eq. 9} & Est. Int. & $8.164992 \mathrm{e}-05$ & $2.284723 \mathrm{e}-04$ & $1.227586 \mathrm{e}-04$ & $7.442899 \mathrm{e}-10$ \\
\hline & Est. Dir. & $1.15 \xi$ & $3.666685 \mathrm{e}-03$ & $2.335491 \mathrm{e}-03$ & $6.590719 \mathrm{e}-07$ \\
\hline \multirow[t]{2}{*}{ Eq. 10a } & Est. Int. & 2.020 & 3.91 & $4.764461 \mathrm{e}-05$ & $1.805229 \mathrm{e}-09$ \\
\hline & Est. Dir. & 2.630 & $8.40842 \mathrm{e}-04$ & $1.036276 \mathrm{e}-03$ & $5.854524 \mathrm{e}-08$ \\
\hline \multirow[t]{2}{*}{ Eq. 10b } & Est. Int. & $4.967675 \mathrm{e}-01$ & $9.499558 \mathrm{e}-04$ & $4.55977 \mathrm{e}-02$ & $2.262022 \mathrm{e}-04$ \\
\hline & Est. Dir. & $2.130602 \mathrm{e}-01$ & $3.943831 \mathrm{e}-04$ & $2.034254 \mathrm{e}-02$ & $1.280671 \mathrm{e}-05$ \\
\hline \multirow[t]{2}{*}{ Eq. 11a } & Est. Int. & 8.24 & 1.28 & $1.883151 \mathrm{e}-04$ & $5.892836 \mathrm{e}-09$ \\
\hline & Est. Dir. & 3.959 & $1.16757 \mathrm{e}-03$ & 7.392867e-03 & $1.870725 \mathrm{e}-05$ \\
\hline \multirow[t]{2}{*}{ Eq. 11b } & Est. Int. & $8.820707 \mathrm{e}-05$ & $1.281126 \mathrm{e}-04$ & $1.874529 \mathrm{e}-04$ & $8.526587 \mathrm{e}-09$ \\
\hline & Est. Dir. & $5.747192 \mathrm{e}-03$ & $1.25035 \mathrm{e}-03$ & $7.689222 \mathrm{e}-03$ & $2.09921 \mathrm{e}-05$ \\
\hline \multirow[t]{2}{*}{ Eq. 11c } & Est. Int. & $5.365138 \mathrm{e}-05$ & $1.338363 \mathrm{e}-04$ & $2.033137 \mathrm{e}-04$ & $2.603827 \mathrm{e}-09$ \\
\hline & Est. Dir. & $7.835532 \mathrm{e}-04$ & $8.149633 \mathrm{e}-04$ & $5.959147 \mathrm{e}-03$ & $1.339166 \mathrm{e}-05$ \\
\hline \multirow[t]{2}{*}{ Eq. $12 \mathrm{a}$} & Est. Int. & $1.605688 \mathrm{e}-01$ & $3.148765 \mathrm{e}-01$ & $8.658627 \mathrm{e}-03$ & $1.209114 \mathrm{e}-03$ \\
\hline & Est. Dir. & $3.742253 \mathrm{e}-03$ & $6.045599 \mathrm{e}-03$ & $4.171266 \mathrm{e}-04$ & $5.593064 \mathrm{e}-07$ \\
\hline \multirow[t]{2}{*}{ Eq. 12b } & Est. Int. & $4.00806 \mathrm{e}-04$ & $3.217966 \mathrm{e}-04$ & $1.523761 \mathrm{e}-04$ & $3.716994 \mathrm{e}-09$ \\
\hline & Est. Dir. & $2.45737 \mathrm{e}-05$ & $1.123774 \mathrm{e}-04$ & $8.652959 \mathrm{e}-05$ & $1.127927 \mathrm{e}-07$ \\
\hline \multirow[t]{2}{*}{ Eq. 12c } & Est. Int. & $2.348892 \mathrm{e}-04$ & $1.686582 \mathrm{e}-03$ & $3.358471 \mathrm{e}-04$ & $4.998449 \mathrm{e}-08$ \\
\hline & Est. Dir. & $1.272234 \mathrm{e}-03$ & $4.184619 \mathrm{e}-03$ & $5.919095 \mathrm{e}-04$ & $4.029824 \mathrm{e}-05$ \\
\hline
\end{tabular}

Tabela 6.19: Erros quadráticos para soluções com ruído 3, 100 ruídos 


\begin{tabular}{|c|c|c|c|c|c|}
\hline & & $\mathbf{F}$ & $\mathrm{G}$ & $\mathbf{H}$ & $\mathbf{K}$ \\
\hline \multirow[t]{2}{*}{ Eq. 1} & & $1.801946 \mathrm{e}-06$ & 7.952995e-06 & $1.053754 \mathrm{e}-05$ & $2.591296 \mathrm{e}-10$ \\
\hline & Est. Dir. & $2.02097 \mathrm{e}-05$ & $8.914453 \mathrm{e}-05$ & $8.748549 \mathrm{e}-05$ & $4.257607 \mathrm{e}-09$ \\
\hline \multirow[t]{2}{*}{ Eq. 2} & Est. Int. & $6.341354 \mathrm{e}-08$ & $1.469161 \mathrm{e}-06$ & $1.812247 \mathrm{e}-05$ & $6.902745 \mathrm{e}-10$ \\
\hline & Est. Dir. & $9.473544 \mathrm{e}-07$ & $9.563341 \mathrm{e}-06$ & $8.274548 \mathrm{e}-05$ & $4.577688 \mathrm{e}-09$ \\
\hline \multirow[t]{2}{*}{ Eq. 3} & Est. Int. & $4.740047 \mathrm{e}-06$ & $2.561346 \mathrm{e}-06$ & $8.801184 \mathrm{e}-06$ & $2.086089 \mathrm{e}-09$ \\
\hline & Est. Dir. & $9.80 \varepsilon$ & $1.299566 \mathrm{e}-04$ & $1.346986 \mathrm{e}-04$ & 4.10 \\
\hline \multirow[t]{2}{*}{ Eq. 4} & Est. Int. & $4.213405 \mathrm{e}-07$ & $3.650938 \mathrm{e}-06$ & $2.064795 \mathrm{e}-06$ & $5.088041 \mathrm{e}-10$ \\
\hline & Est. Dir. & $4.253981 \mathrm{e}-06$ & $2.388761 \mathrm{e}-05$ & $1.055474 \mathrm{e}-05$ & $3.460759 \mathrm{e}-09$ \\
\hline \multirow[t]{2}{*}{ Eq. 5} & Est. Int. & $8.420097 \mathrm{e}-06$ & 3.468 & $1.731383 \mathrm{e}-06$ & $1.032888 \mathrm{e}-10$ \\
\hline & Est. Dir. & 6.976 & 2.99 & $1.626347 \mathrm{e}-05$ & 1.49 \\
\hline \multirow[t]{2}{*}{ Eq. 6} & Est. Int. & $5.153187 \mathrm{e}-06$ & $9.600204 \mathrm{e}-07$ & $6.958802 \mathrm{e}-05$ & $7.961388 \mathrm{e}-09$ \\
\hline & Est. Dir. & $1.497372 \mathrm{e}-04$ & $2.539933 \mathrm{e}-05$ & $3.269115 \mathrm{e}-03$ & $1.28443 \mathrm{e}-05$ \\
\hline \multirow[t]{2}{*}{ Eq. 7} & Est. Int. & 1.40 & 3.119 & 1.568 & 7.66 \\
\hline & Est. Dir. & 2.932 & 7.72 & 3.56 & $5 e-06$ \\
\hline \multirow[t]{2}{*}{ Eq. 8} & Est. Int. & 6.908 & 9.238 & $1.767818 \mathrm{e}-05$ & $6.820261 \mathrm{e}-08$ \\
\hline & Est. Dir. & $53 \mathrm{e}-05$ & 1.227 & $2.993827 \mathrm{e}-04$ & $6.712853 \mathrm{e}-07$ \\
\hline \multirow[t]{2}{*}{ Eq. 9} & Est. Int. & 6.583 & 1.788 & 9.284 & $1.147045 \mathrm{e}-10$ \\
\hline & Est. Dir. & 1.211 & 2.640 & 3.016 & $77 \mathrm{e}-07$ \\
\hline \multirow[t]{2}{*}{ Eq. 10a } & Est. Int. & 1.499 & 1.060 & $1.064597 \mathrm{e}-05$ & $5.806316 \mathrm{e}-10$ \\
\hline & Est. Dir. & 3.082 & 5.370 & $8.085437 \mathrm{e}-05$ & $1.049524 \mathrm{e}-08$ \\
\hline \multirow[t]{2}{*}{ Eq. 10b } & Est. Int. & 2.500 & 4.86 & $77 \mathrm{e}-03$ & $7.500012 \mathrm{e}-06$ \\
\hline & Est. Dir. & 3.936 & 6.943 & $3.691538 \mathrm{e}-02$ & $3.279627 \mathrm{e}-04$ \\
\hline \multirow[t]{2}{*}{ Eq. 11a } & Est. Int. & $1.077013 \mathrm{e}-05$ & $8.601361 \mathrm{e}-06$ & $2.794482 \mathrm{e}-05$ & $3.168354 \mathrm{e}-09$ \\
\hline & Est. Dir. & 6.1610 & 1.665 & $1.348528 \mathrm{e}-03$ & $1.556632 \mathrm{e}-06$ \\
\hline \multirow[t]{2}{*}{ Eq. 11b } & Est. Int. & $1.149797 \mathrm{e}-05$ & 8.582 & $2.784755 \mathrm{e}-05$ & $5.43872 \mathrm{e}-09$ \\
\hline & Est. Dir. & $8.822403 \mathrm{e}-04$ & $1.73022 \mathrm{e}-04$ & $1.389902 \mathrm{e}-03$ & $1.78861 \mathrm{e}-06$ \\
\hline \multirow[t]{2}{*}{ Eq. 11c } & Est. Int. & $7.083404 \mathrm{e}-06$ & $9.04211 \mathrm{e}-06$ & $2.971144 \mathrm{e}-05$ & $1.82783 \mathrm{e}-09$ \\
\hline & & $1.370687 \mathrm{e}-04$ & 1.261 & $1.102524 \mathrm{e}-03$ & $4.76974 \mathrm{e}-07$ \\
\hline \multirow[t]{2}{*}{ Eq. $12 \mathrm{a}$} & Est. Int. & $2.775783 \mathrm{e}-02$ & $5.477691 \mathrm{e}-02$ & $1.582562 \mathrm{e}-03$ & $2.316789 \mathrm{e}-04$ \\
\hline & Est. Dir. & $5.541562 \mathrm{e}-05$ & $1.173161 \mathrm{e}-04$ & $1.47726 \mathrm{e}-05$ & $4.131038 \mathrm{e}-06$ \\
\hline \multirow[t]{2}{*}{ Eq. $12 b$} & Est. Int. & $3.962721 \mathrm{e}-04$ & $3.290023 \mathrm{e}-04$ & $1.892457 \mathrm{e}-04$ & $4.029867 \mathrm{e}-09$ \\
\hline & Est. Dir. & $4.654029 \mathrm{e}-06$ & $1.903055 \mathrm{e}-05$ & $1.455106 \mathrm{e}-05$ & $1.414924 \mathrm{e}-07$ \\
\hline \multirow[t]{2}{*}{ Eq. 12c } & Est. Int. & $2.542911 \mathrm{e}-04$ & $1.978797 \mathrm{e}-03$ & $3.727129 \mathrm{e}-04$ & $8.983911 \mathrm{e}-08$ \\
\hline & Est. Dir. & $1.725855 \mathrm{e}-04$ & $5.539705 \mathrm{e}-04$ & $6.519018 \mathrm{e}-05$ & $2.064428 \mathrm{e}-06$ \\
\hline
\end{tabular}

Tabela 6.20: Erros quadráticos para soluções com ruído 3, 1000 ruídos 


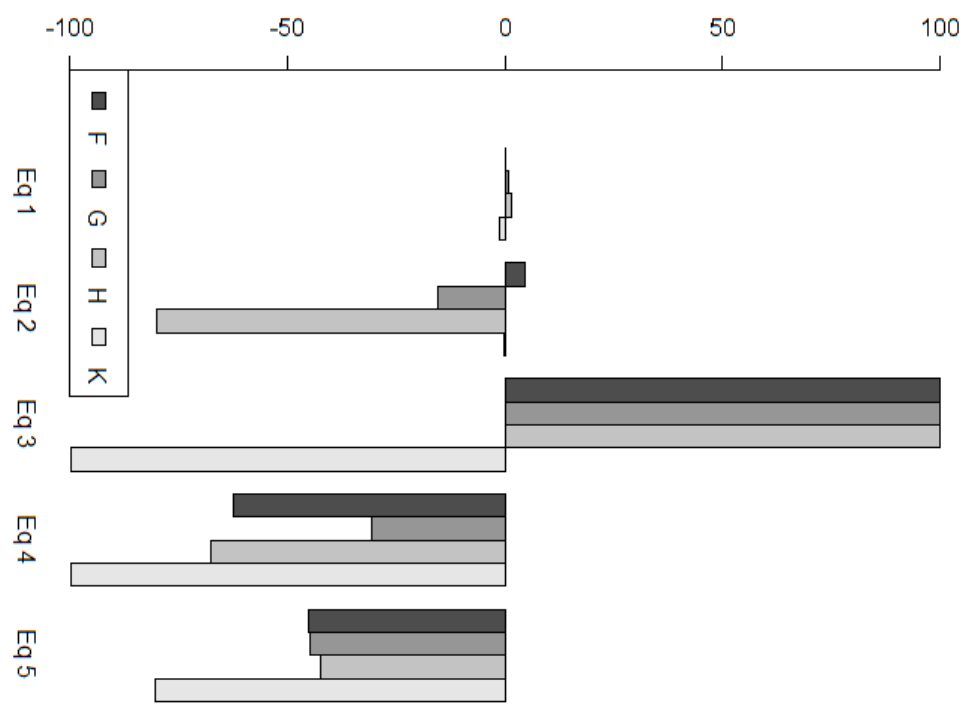

茴

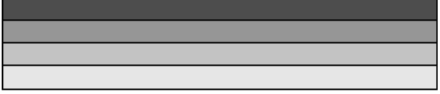

四

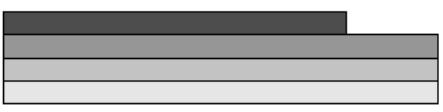

$\frac{m}{\infty}$

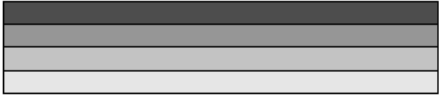

$\frac{\pi}{6}$

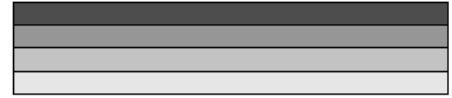

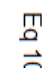

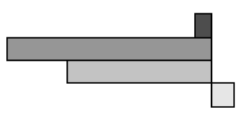

四

의

茴

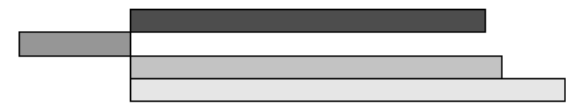

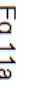

$\stackrel{\text { 定 }}{\overrightarrow{\vec{\sigma}}}$

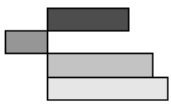

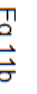

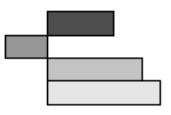

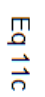

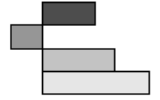

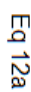

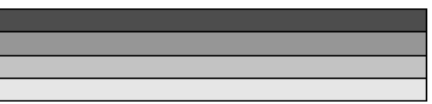

$\frac{\pi}{\vec{V}}$

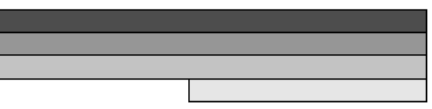

I

00 

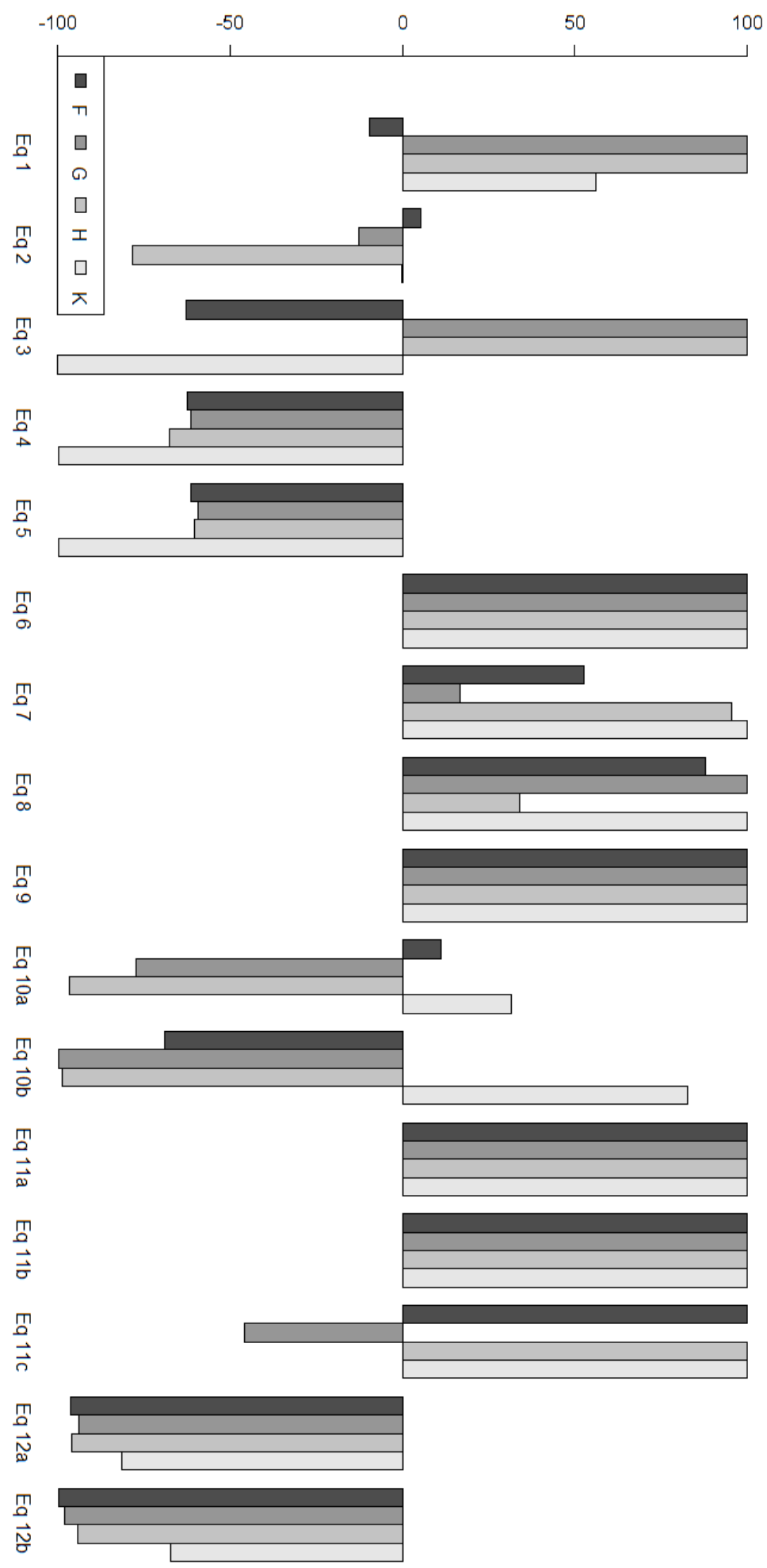

$\stackrel{\text { m }}{\stackrel{0}{n}}$

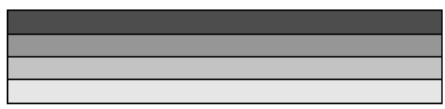

Figura 6.11: Comparação dos erros quadráticos para soluções com ruído sem ruído, usando $t=t_{25}=0,24$ 

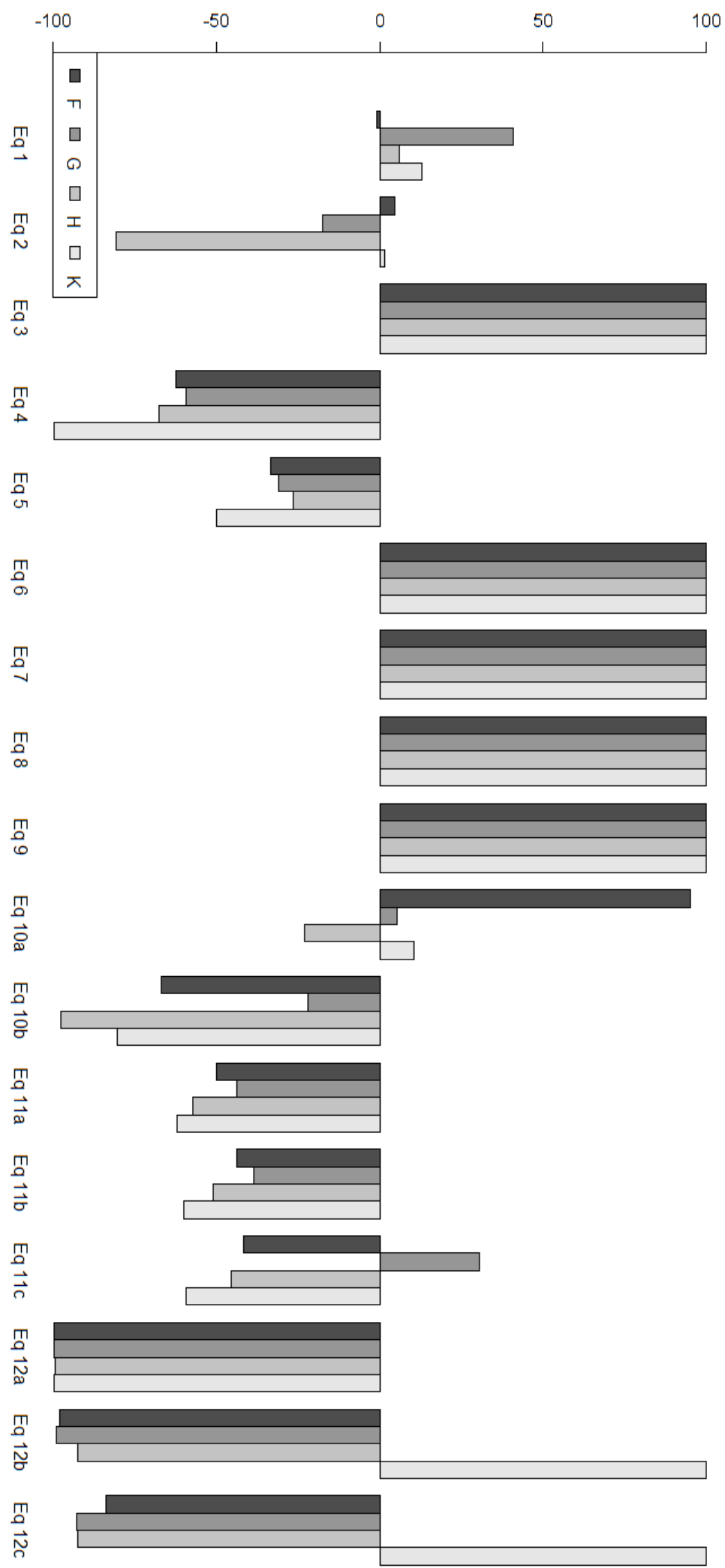

Figura 6.12: Comparação dos erros quadráticos para soluções com ruído sem ruído, usando $t=t_{75}=0,74$ 

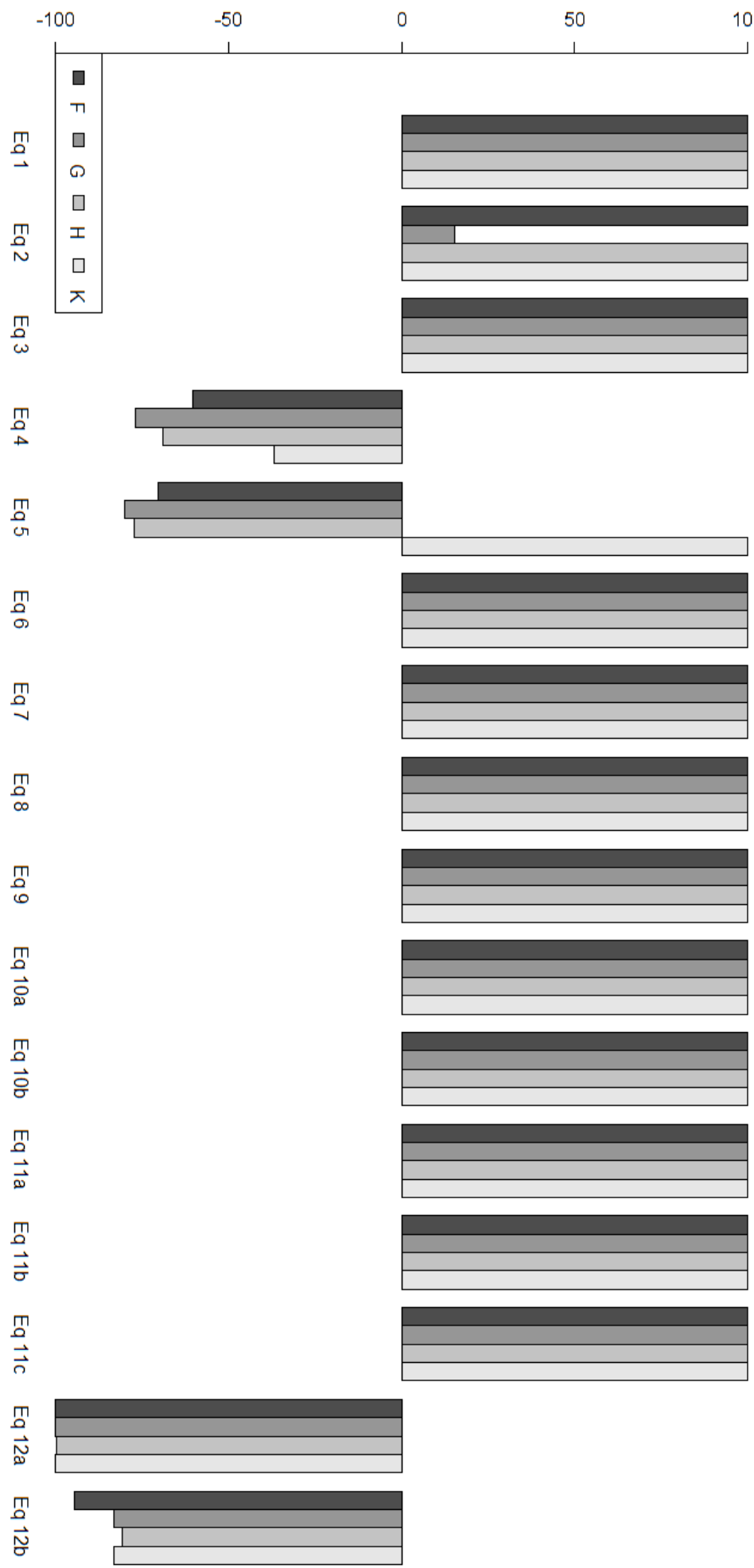

$\stackrel{\text { M }}{\vec{n}}$

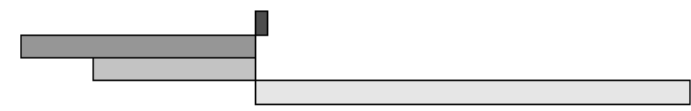

Figura 6.13: Comparação dos erros quadráticos para soluções com ruído 1, 1 ruído 

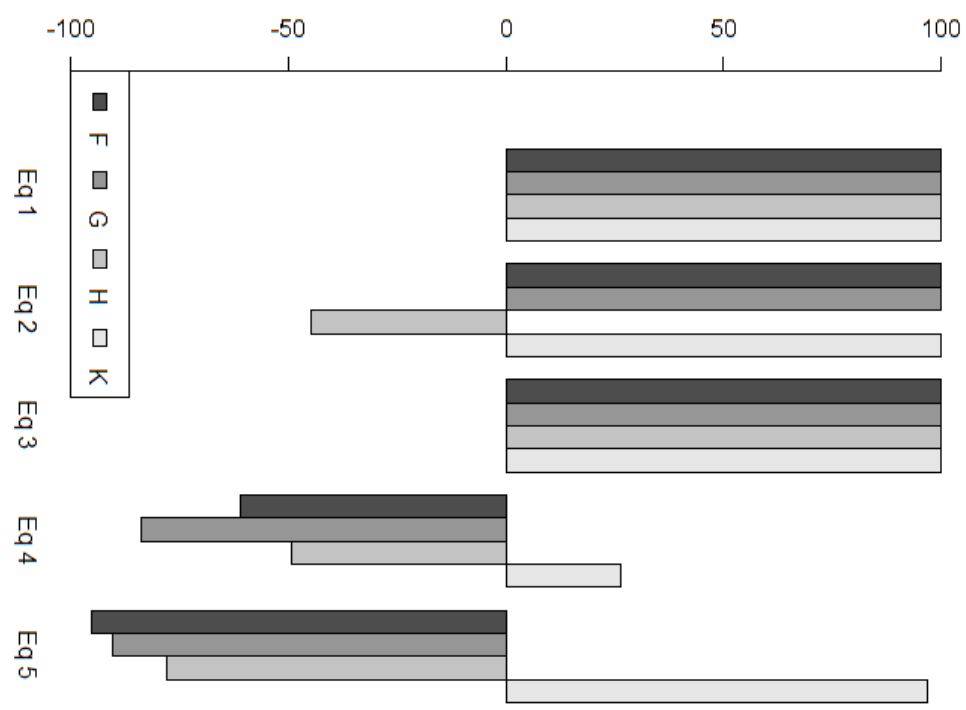

茴

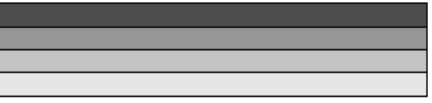

임

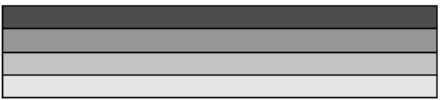

$\frac{m}{\infty}$

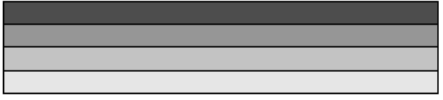

$\frac{\pi}{6}$

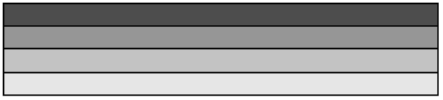

\%

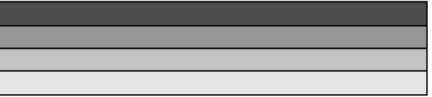

밍

웅

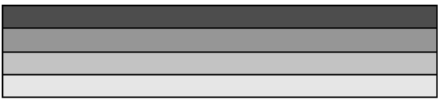

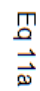

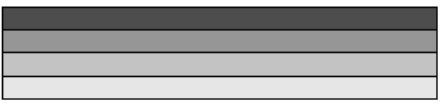

밈

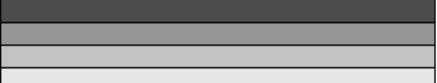

四

$\overrightarrow{\vec{n}}$

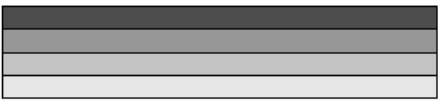

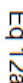

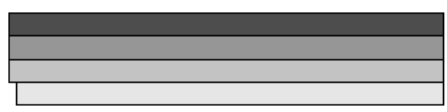

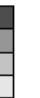

$\frac{\mathbb{N}}{\vec{V}}$

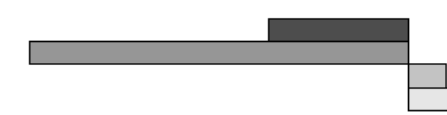

圆

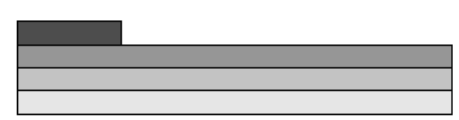

Figura 6.14: Comparação dos erros quadráticos para soluções com ruído 1 , 1 ruído, usando $t=t_{25}=0,24$ 


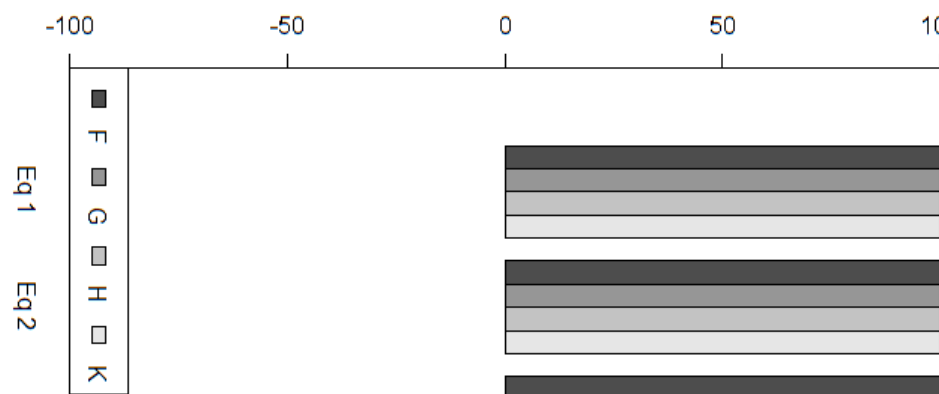

.

$\frac{1}{8}$

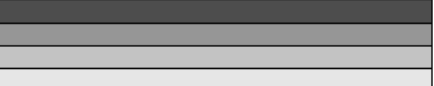

祭

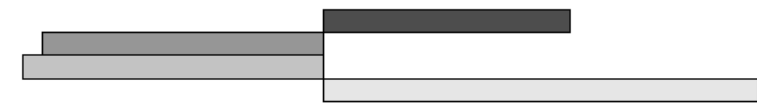

용

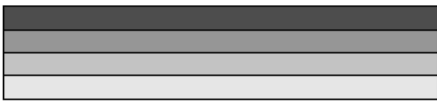

$\stackrel{n}{2}$

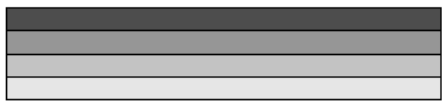

$\underset{\infty}{1}$

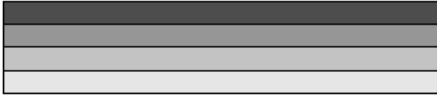

$\frac{1}{6}$

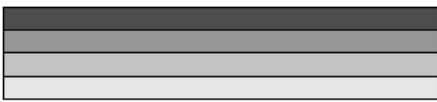

$\stackrel{m}{\stackrel{2}{0}}$

$\vec{a}$

m

$\vec{\sigma}$

$\stackrel{\text { m }}{\overrightarrow{\vec{D}}}$

$\stackrel{\frac{\pi}{\vec{N}}}{\overrightarrow{0}}$
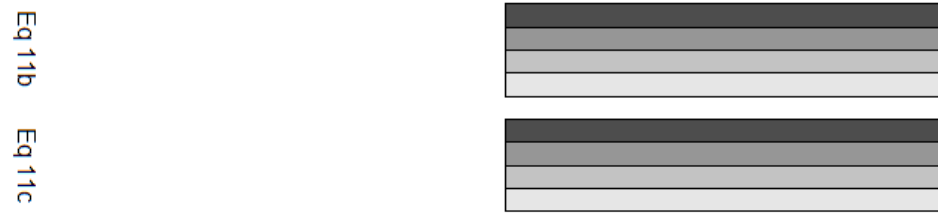

I
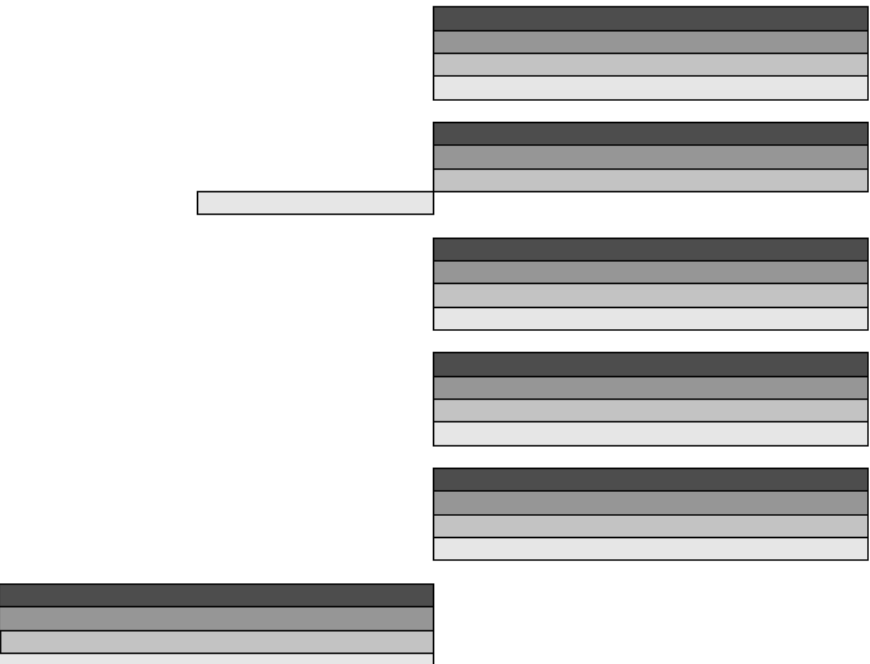

N

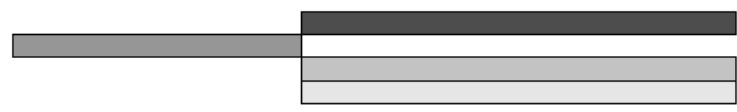

$\stackrel{0}{\Omega}$

\section{0}

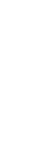



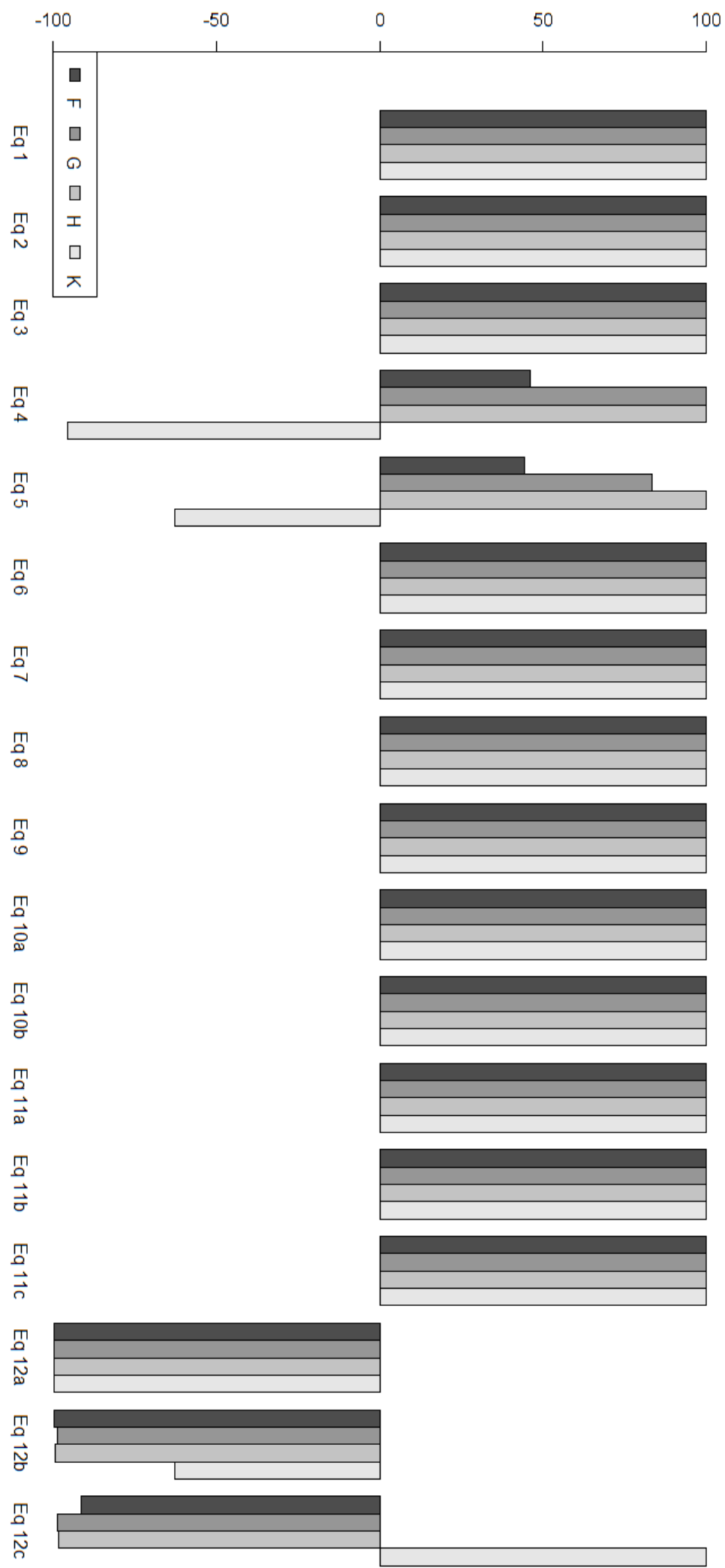

Figura 6.16: Comparação dos erros quadráticos para soluções com ruído 1, 100 ruídos 

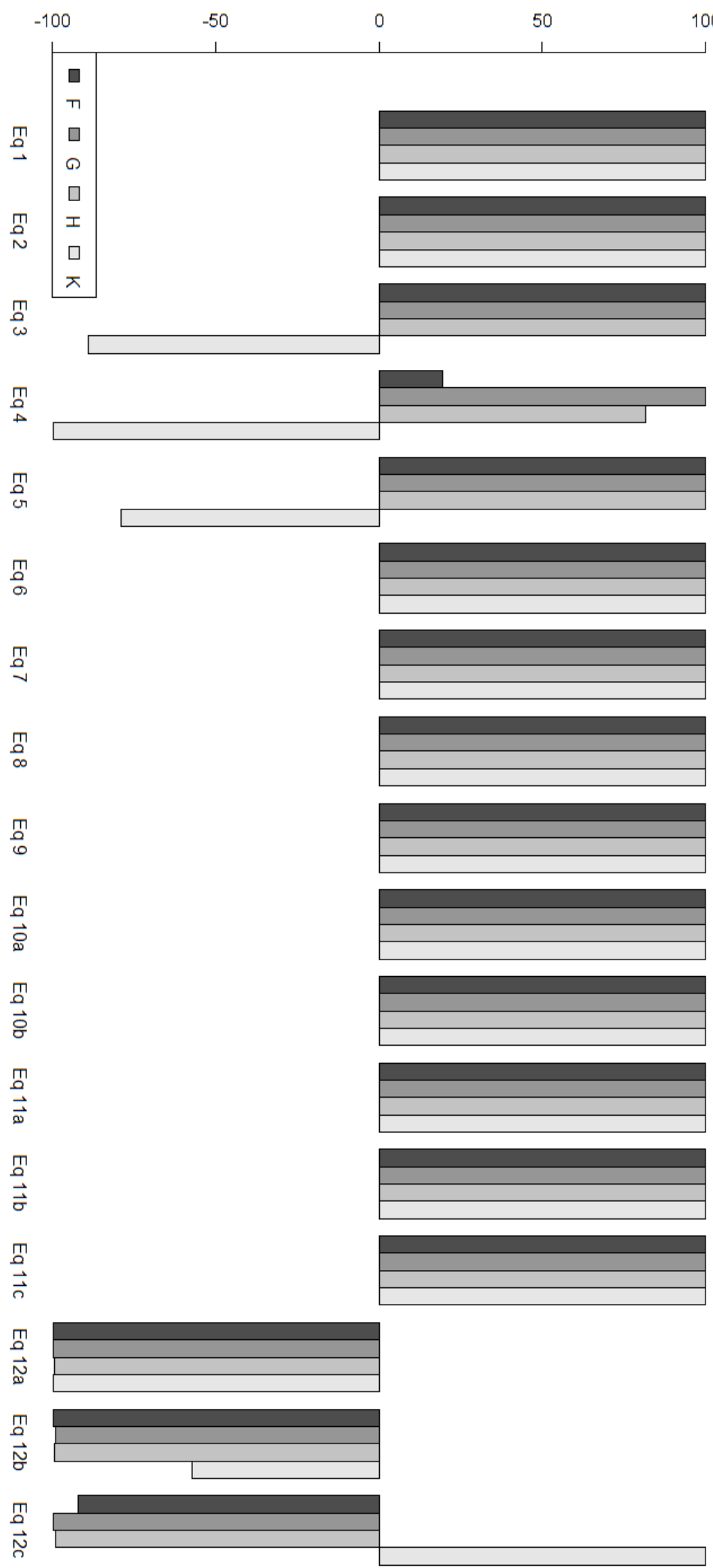

Figura 6.17: Comparação dos erros quadráticos para soluções com ruído 1, 1000 ruídos 


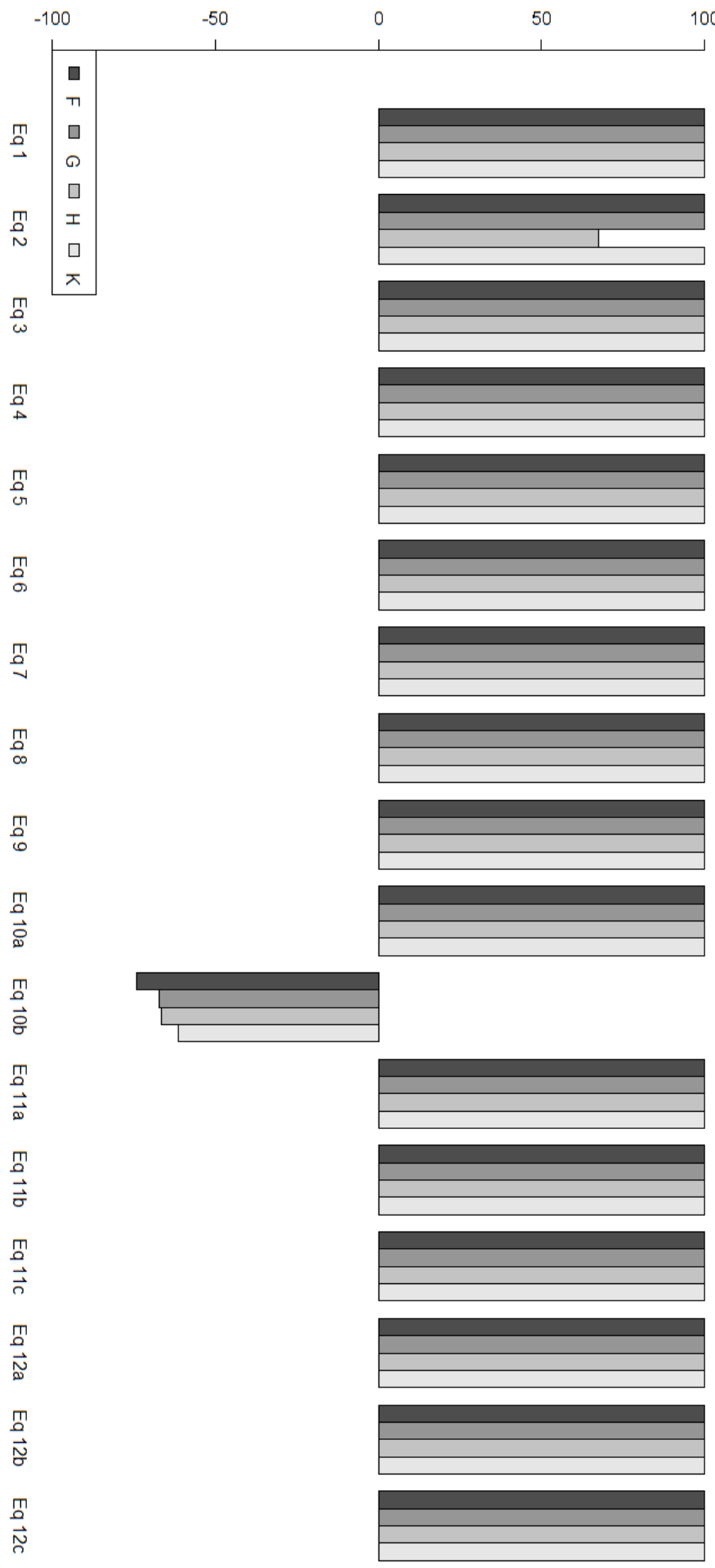

Figura 6.18: Comparação dos erros quadráticos para soluções com ruído 2, 1 ruído 


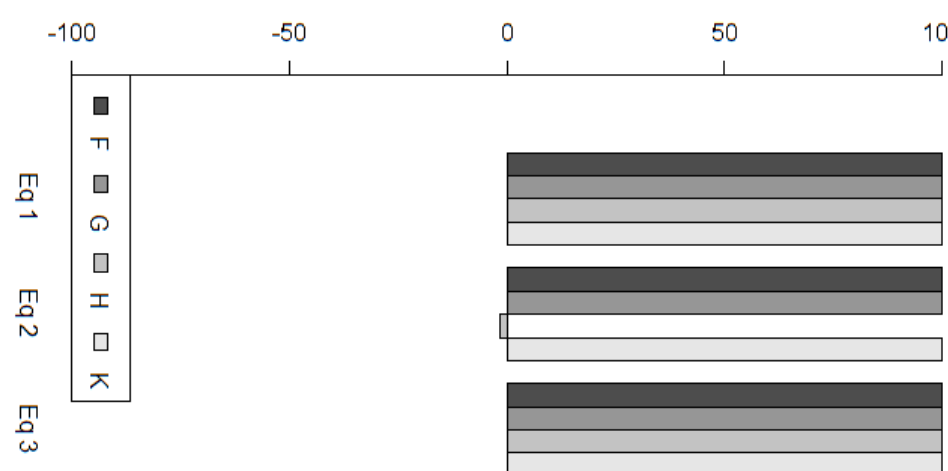

$\frac{1}{\circ}$

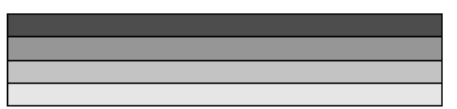

罚

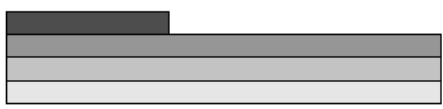

监

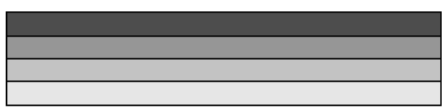

N

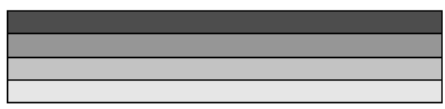

皿

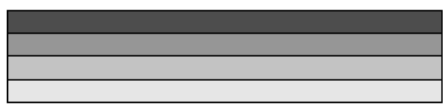

$\frac{1}{6}$

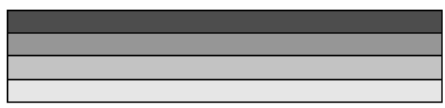

四

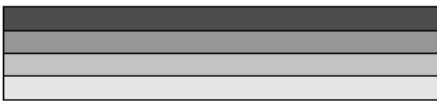

용

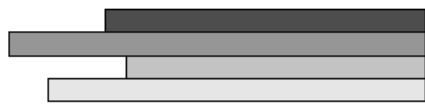

塄

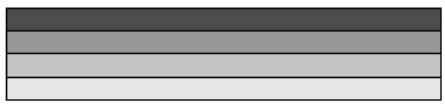

四

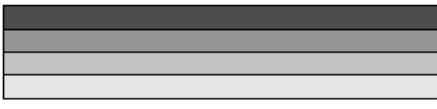

$\stackrel{\substack{0 \\ \vec{n}}}{\vec{n}}$

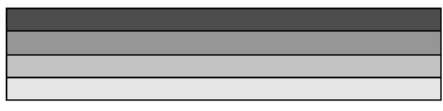

䓠

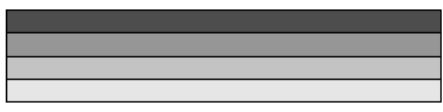

$\frac{N}{\tilde{\sigma}}$

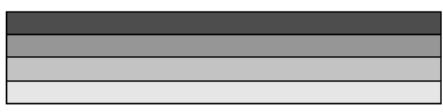

莫

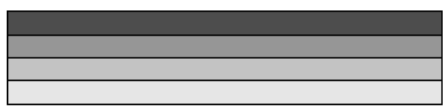

Figura 6.19: Comparação dos erros quadráticos para soluções com ruído 2, 1 ruído, usando $t=t_{25}=0,24$ 


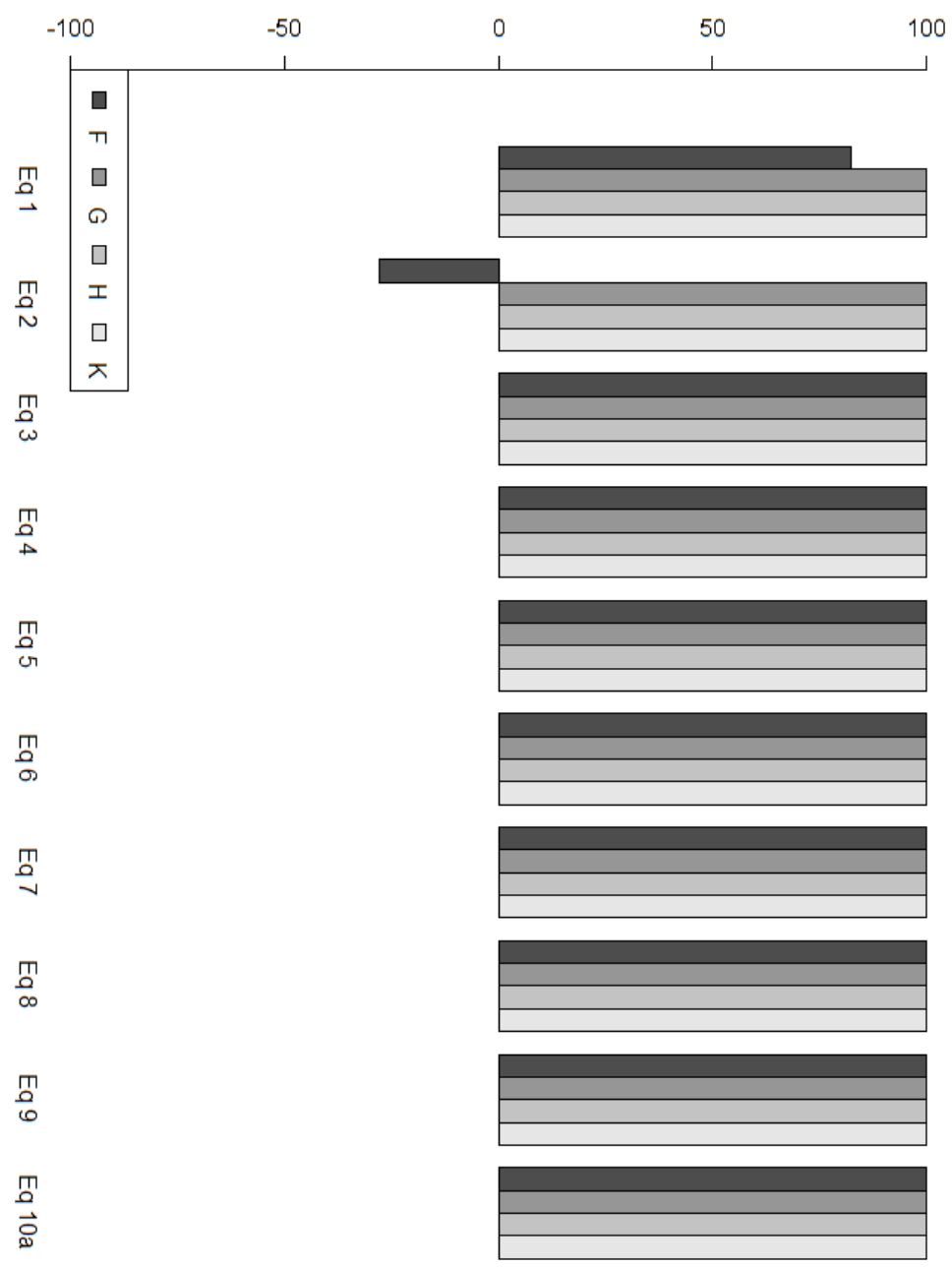

낑

하

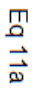

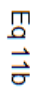

$\stackrel{m}{\stackrel{m}{n}}$
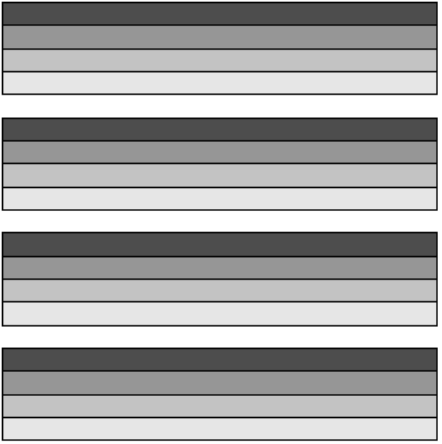

mi

$\vec{\otimes}$

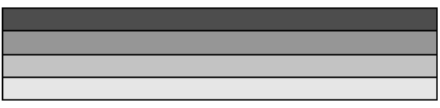

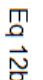

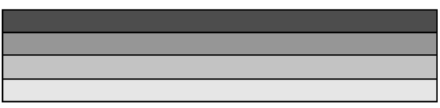

$\stackrel{\Omega}{\Omega}$

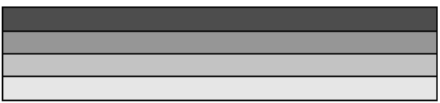

Figura 6.20: Comparação dos erros quadráticos para soluções com ruído 2, 1 ruído, usando $t=t_{75}=0,74$ 


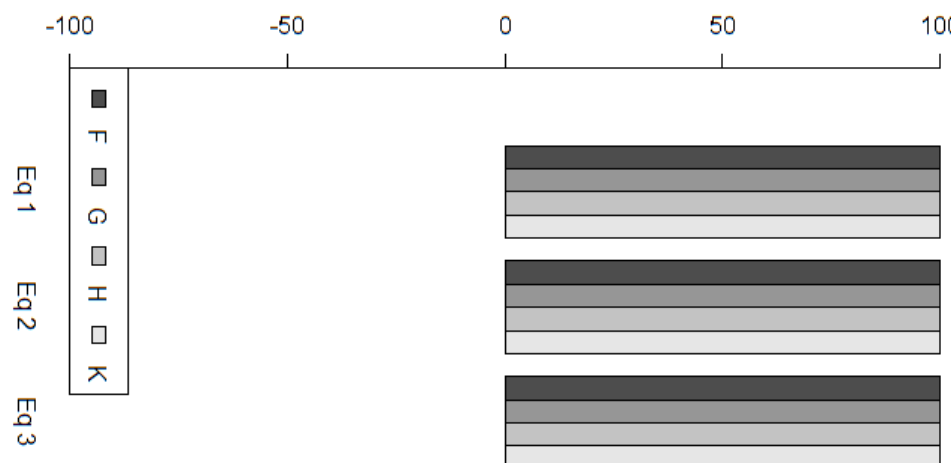

血

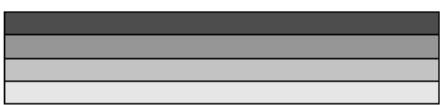

罚

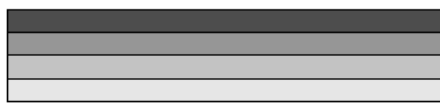

监

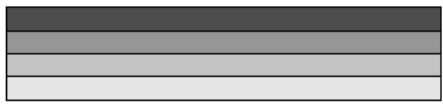

N

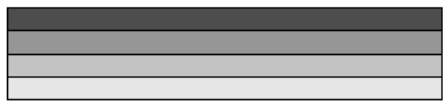

皿

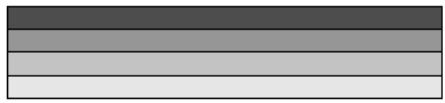

$\frac{\pi}{6}$

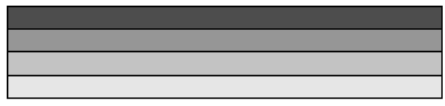

잉

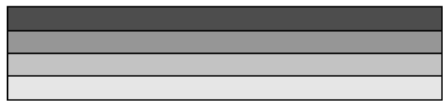

四

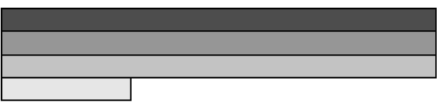

苜

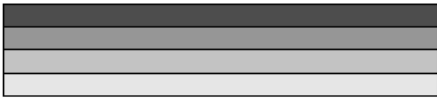

$\frac{\text { m }}{\overrightarrow{\vec{\sigma}}}$

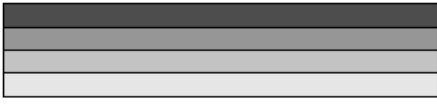

$\stackrel{\text { 监 }}{\vec{\sim}}$

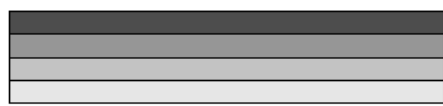

䓠

$\frac{N}{\vec{N}}$
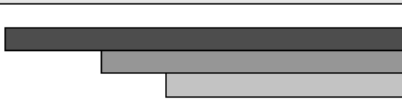

㲾

100 

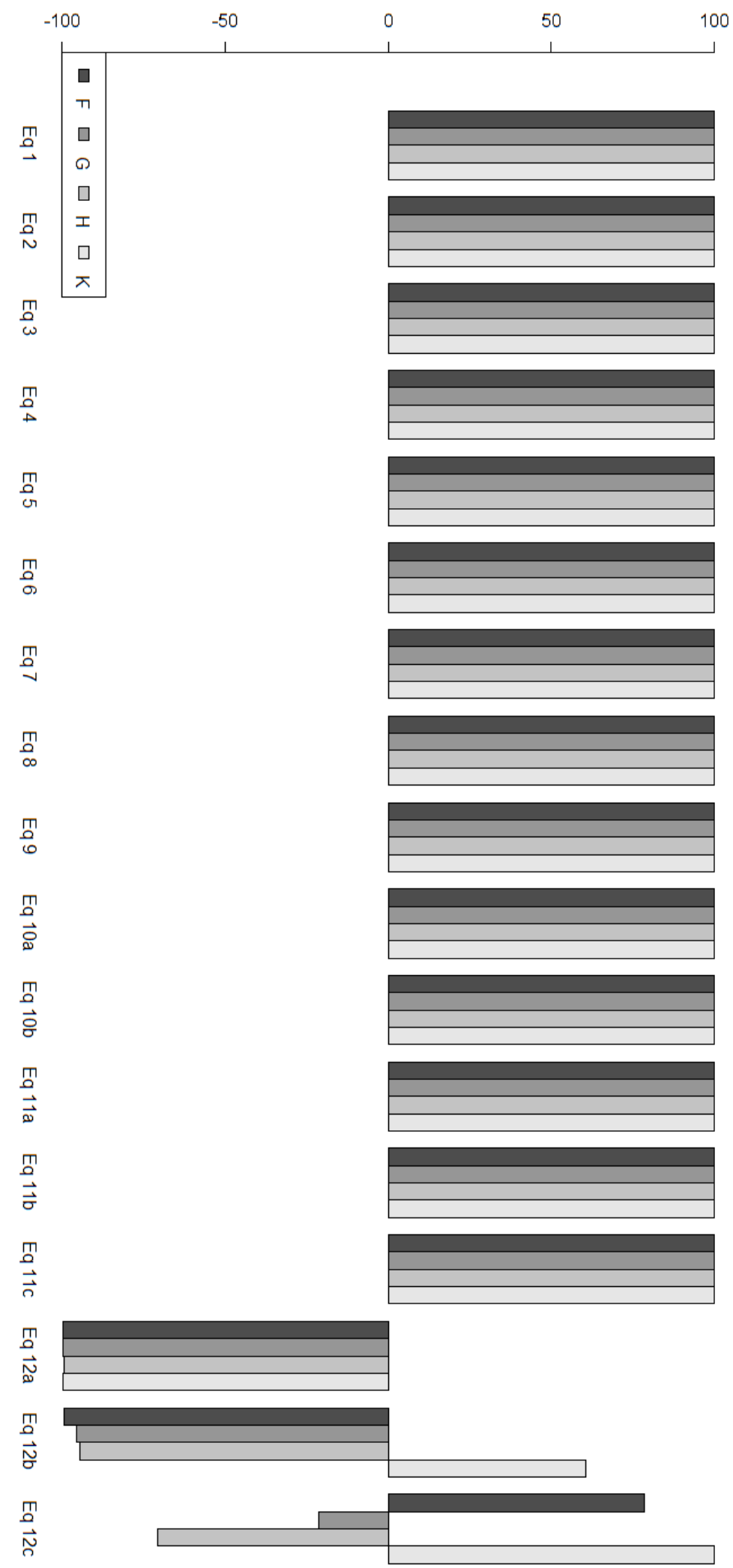

Figura 6.22: Comparação dos erros quadráticos para soluções com ruído 2, 1000 ruídos 


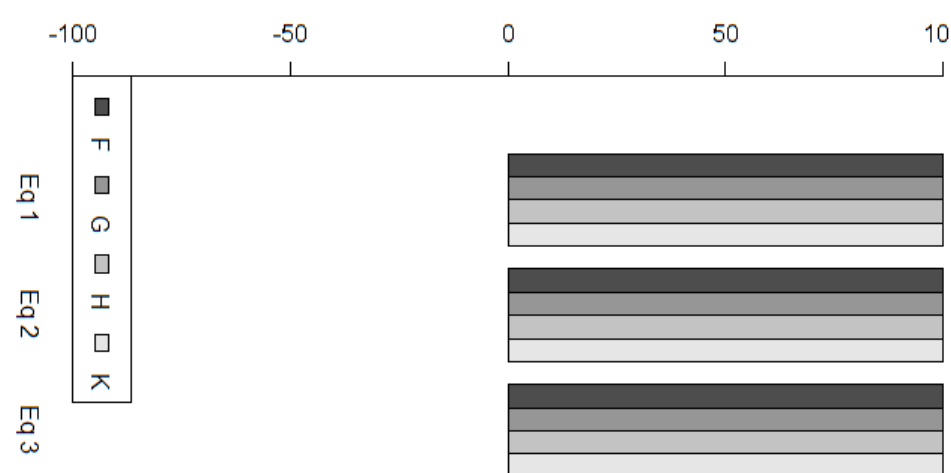

$\frac{1}{\circ}$

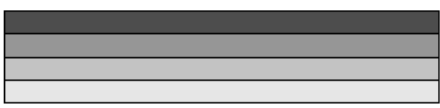

罚

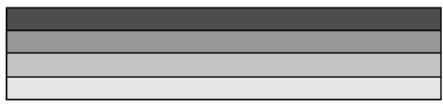

祭

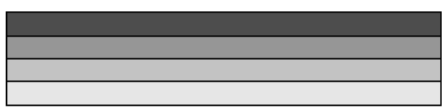

N

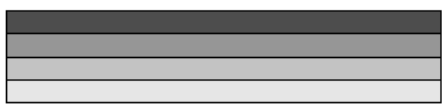

皿

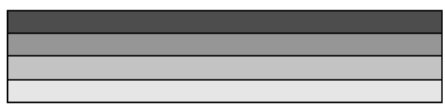

$\frac{1}{6}$

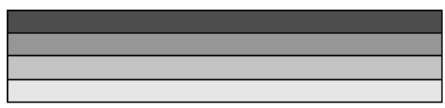

四

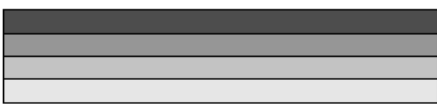

미

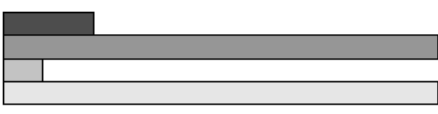

四

$\overrightarrow{\vec{D}}$

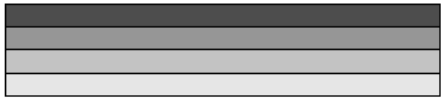

$\stackrel{\text { 品 }}{\overrightarrow{\vec{\sigma}}}$

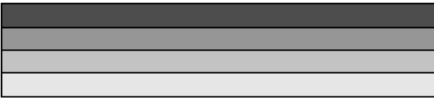

$\stackrel{\substack{n \\ \vec{n}}}{\vec{n}}$

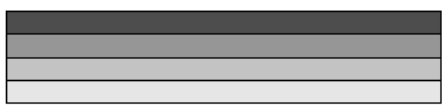

$\frac{\pi}{\mathbb{N}}$

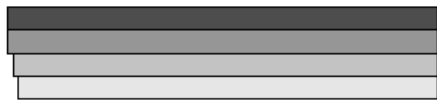

宪

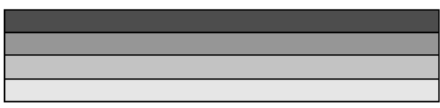

罢

\section{0}




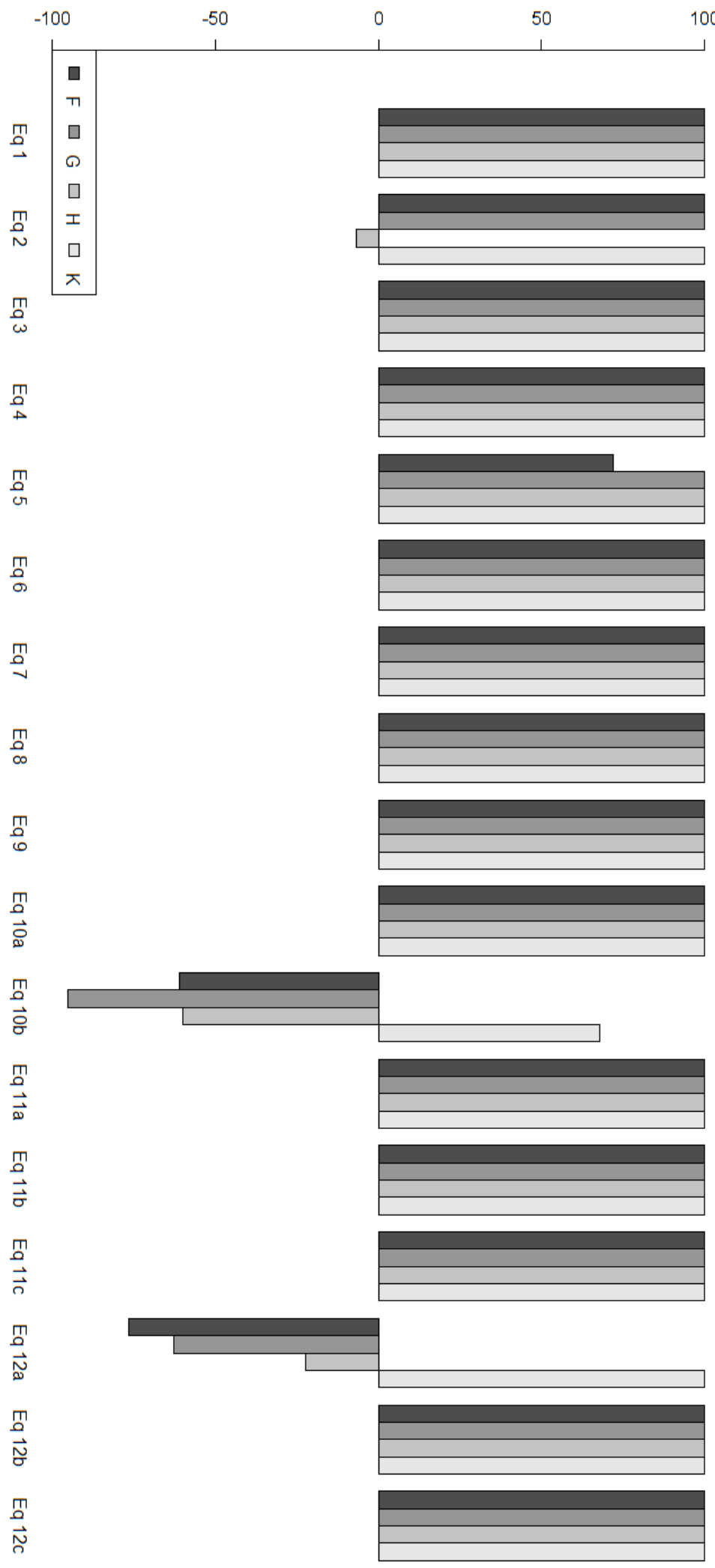

Figura 6.24: Comparação dos erros quadráticos para soluções com ruído 3, 1 ruído, usando $t=t_{25}=0,24$ 

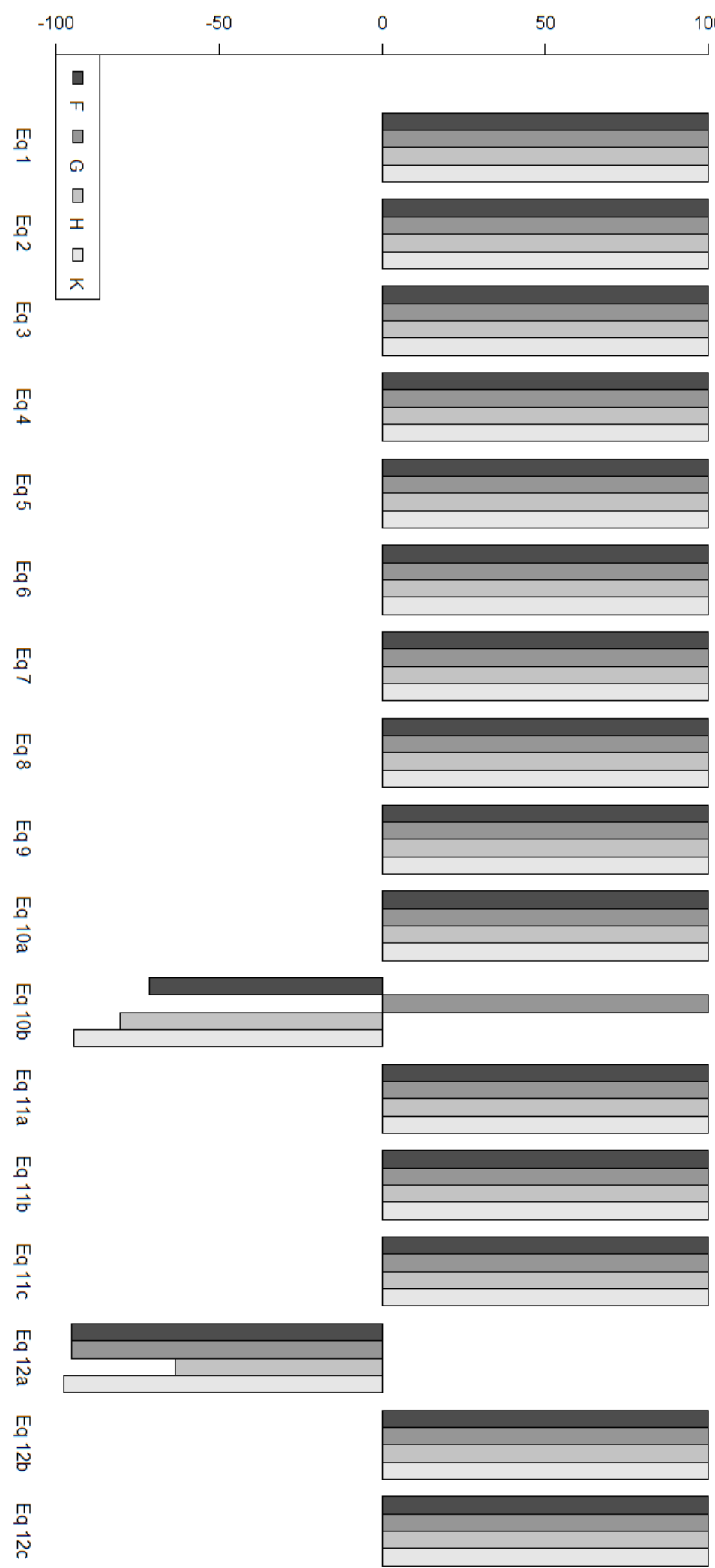

Figura 6.25: Comparação dos erros quadráticos para soluções com ruído 3 , 1 ruído, usando $t=t_{75}=0,74$ 

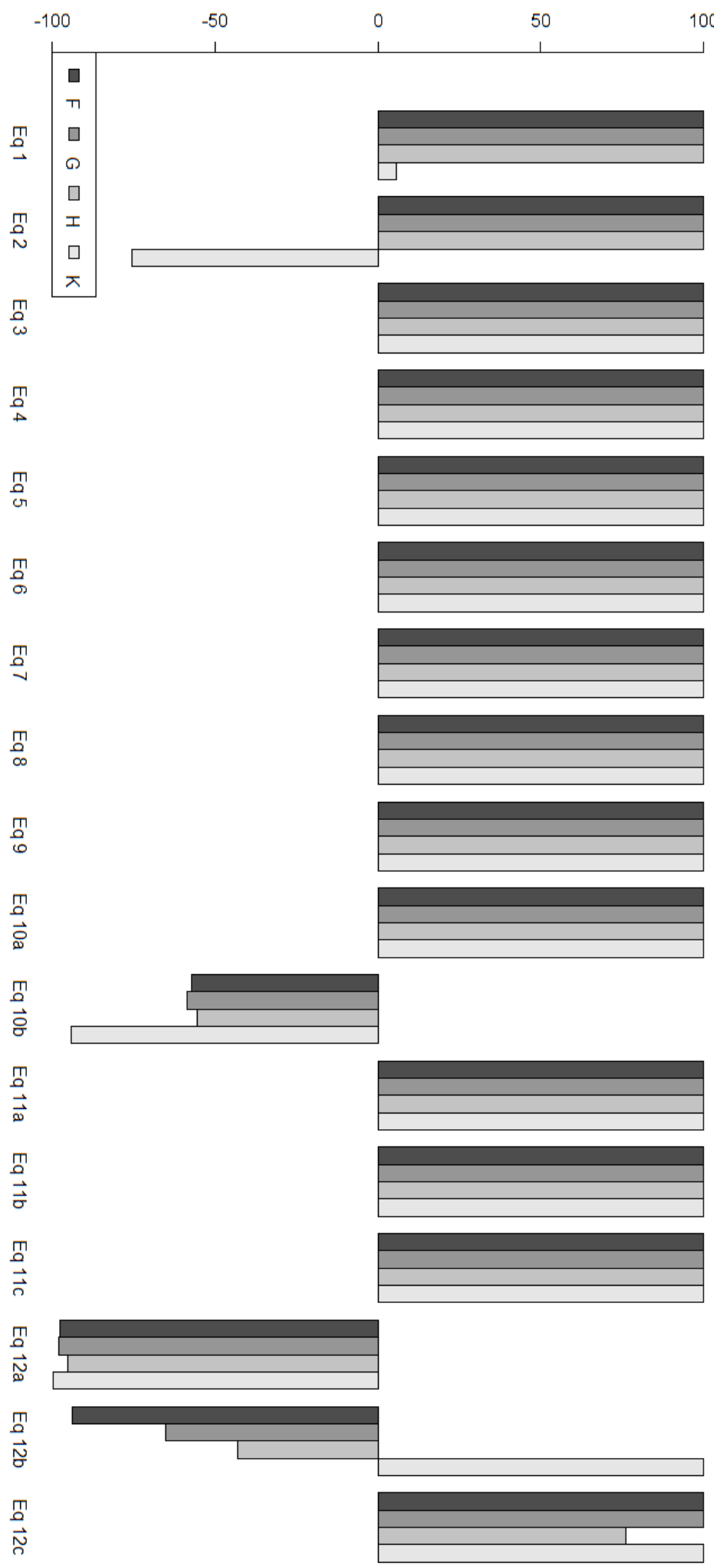

Figura 6.26: Comparação dos erros quadráticos para soluções com ruído 3, 100 ruídos 


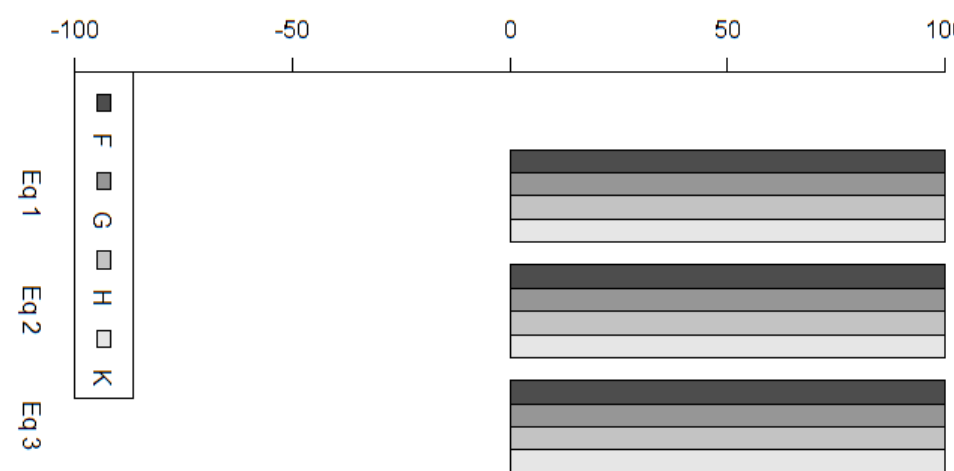

血

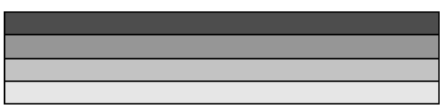

血

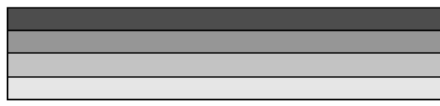

祭

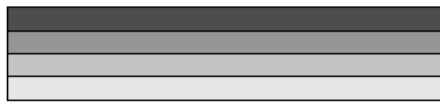

草

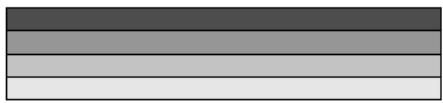

$\underset{\infty}{\infty}$

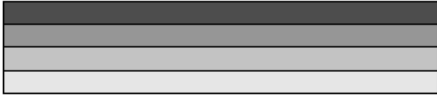

$\frac{\pi}{6}$

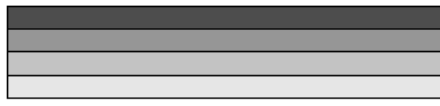

잉

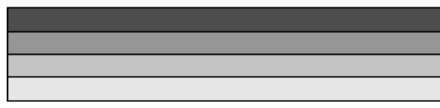

四

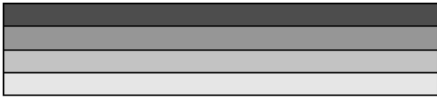

典

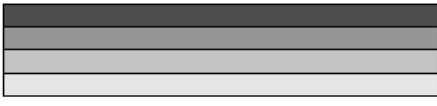

$\stackrel{\text { ग) }}{\overrightarrow{\vec{\sigma}}}$

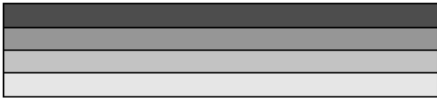

$\stackrel{0}{3}$

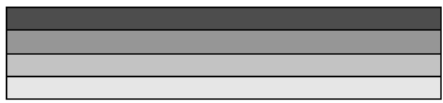

䓠

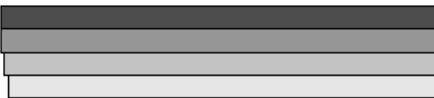

宪

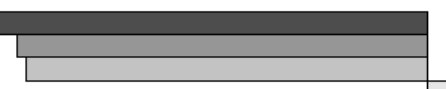

$\stackrel{n}{\sim}$ 

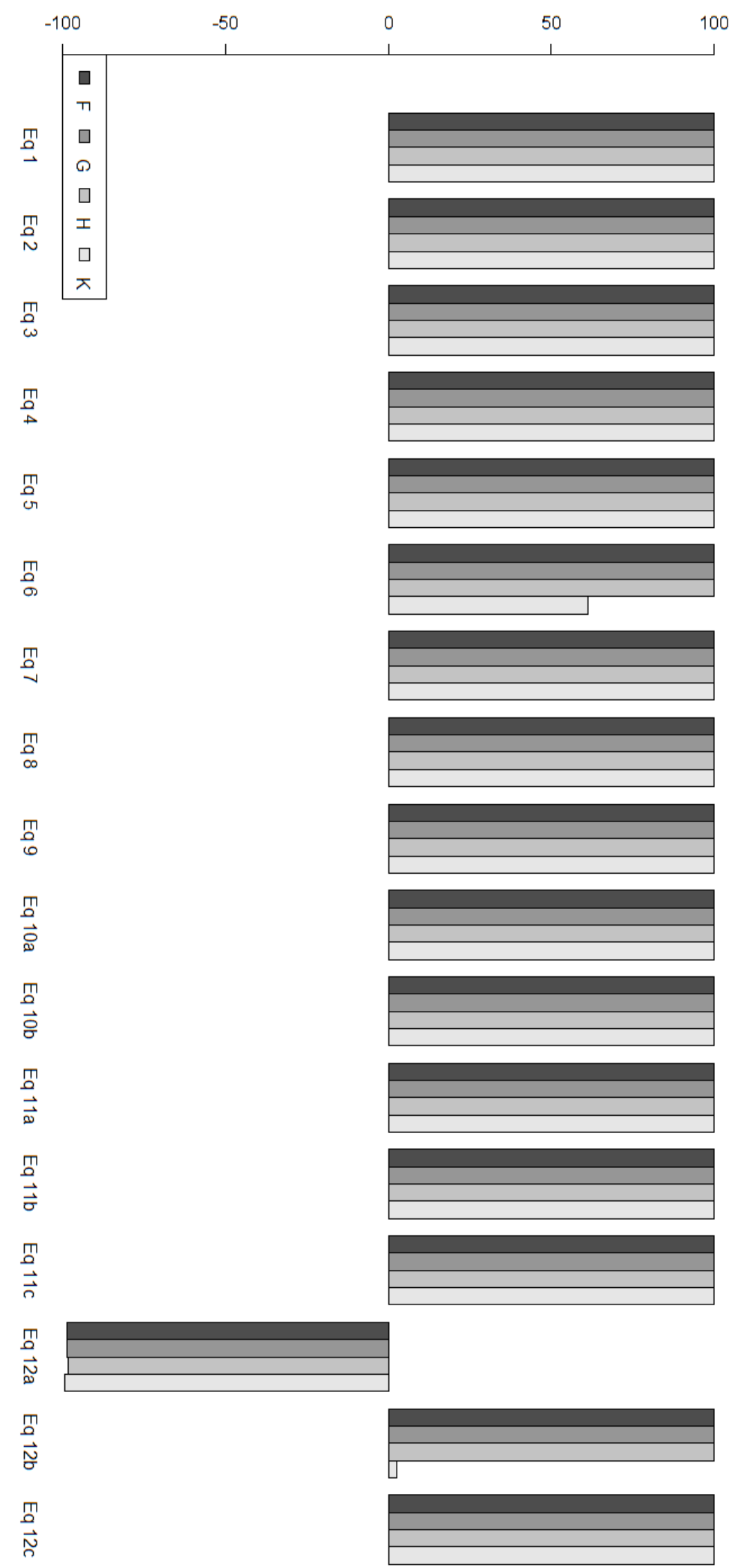

Figura 6.28: Comparação dos erros quadráticos para soluções com ruído 1 e 3, 1 ruído 


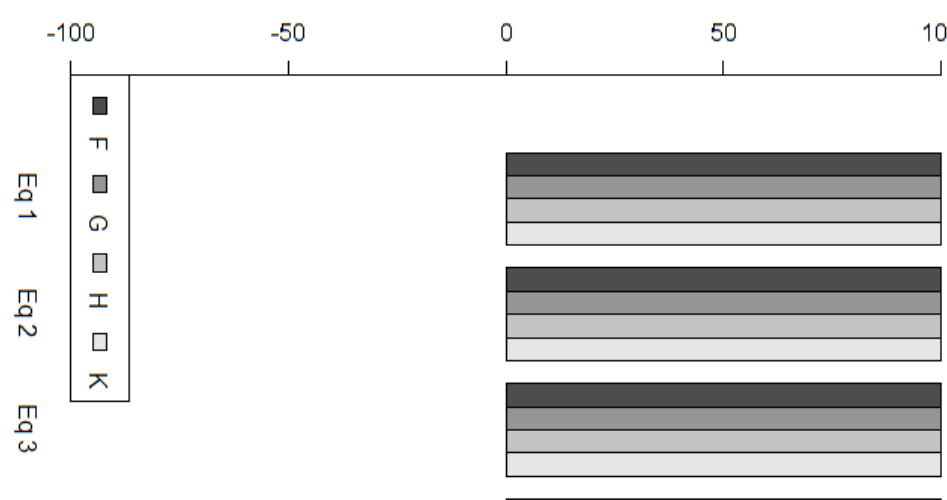

$\stackrel{0}{\circ}$

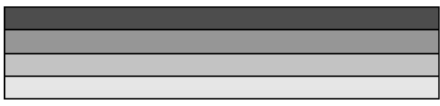

용

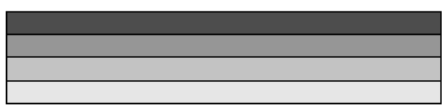

塄

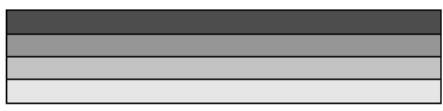

$\stackrel{n}{2}$

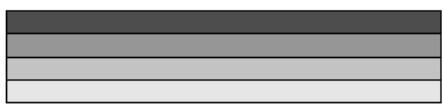

$\underset{\infty}{m}$

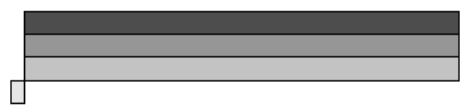

$\frac{1}{0}$

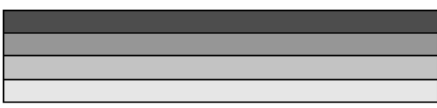

$\stackrel{n}{\circ}$

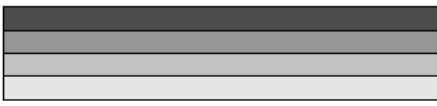

m

$\vec{\sigma}$

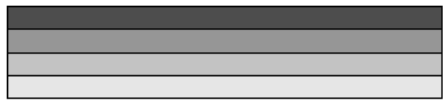

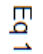

$\overrightarrow{\mathrm{N}}$

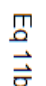

$\stackrel{m}{\stackrel{M}{\vec{D}}}$

$\stackrel{\Pi}{\vec{N}}$
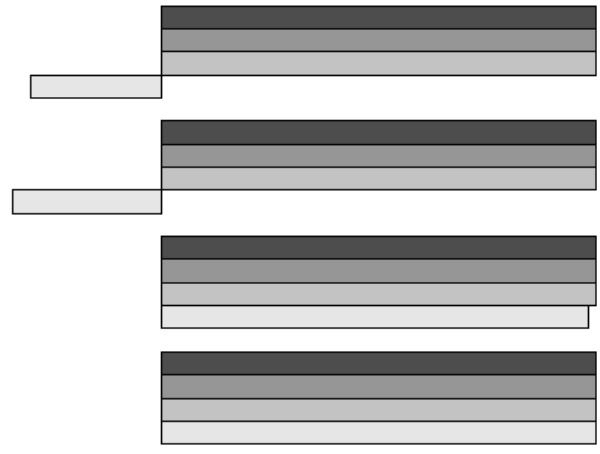

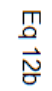

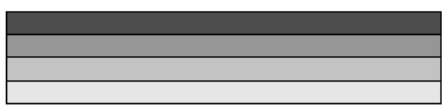

$\stackrel{\Pi}{\Omega}$

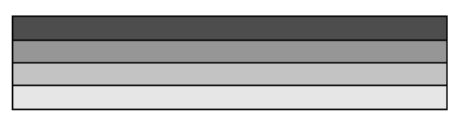

Figura 6.29: Comparação dos erros quadráticos para soluções com ruído 1 e 2, 1 ruído 


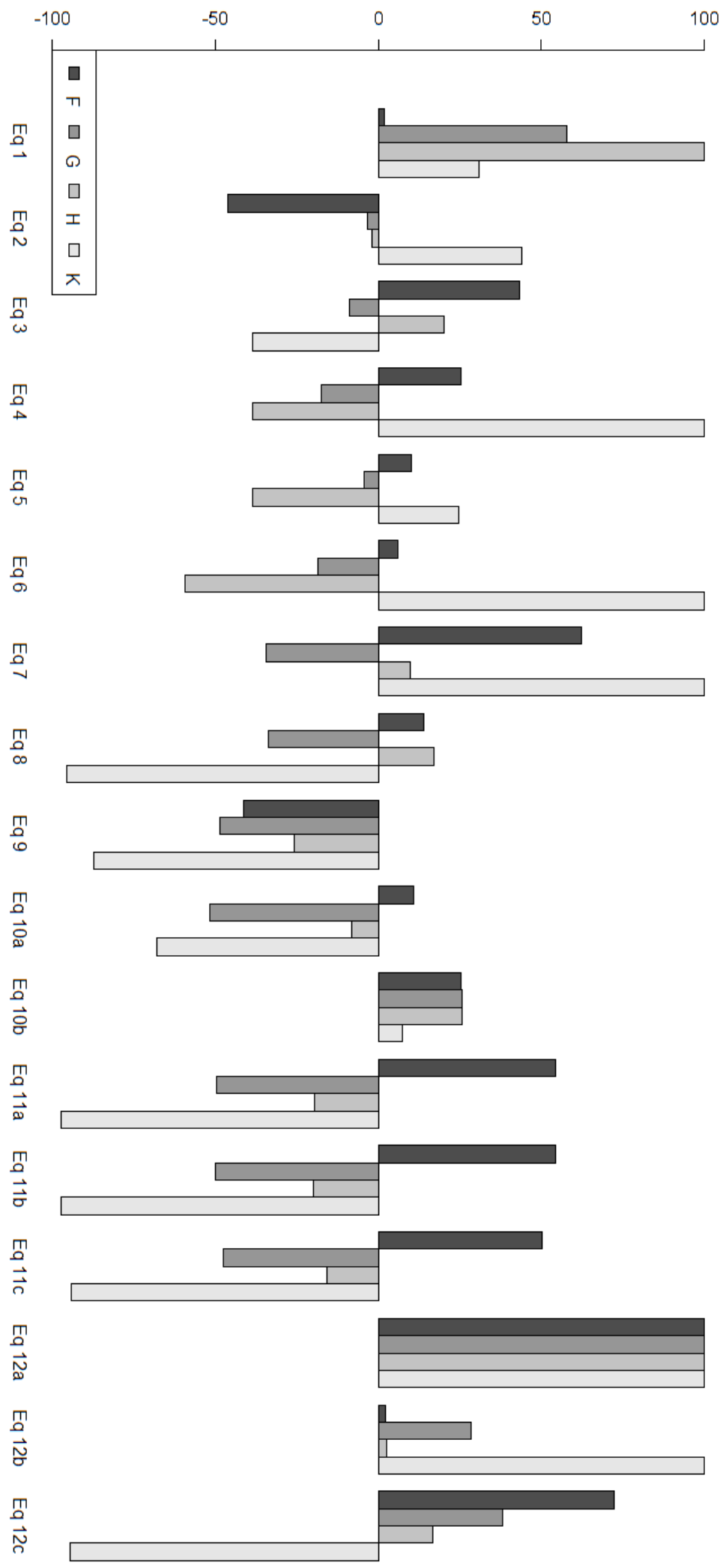

Figura 6.30: Comparação dos erros quadráticos para soluções com ruído 2 e 3, 1 ruído 

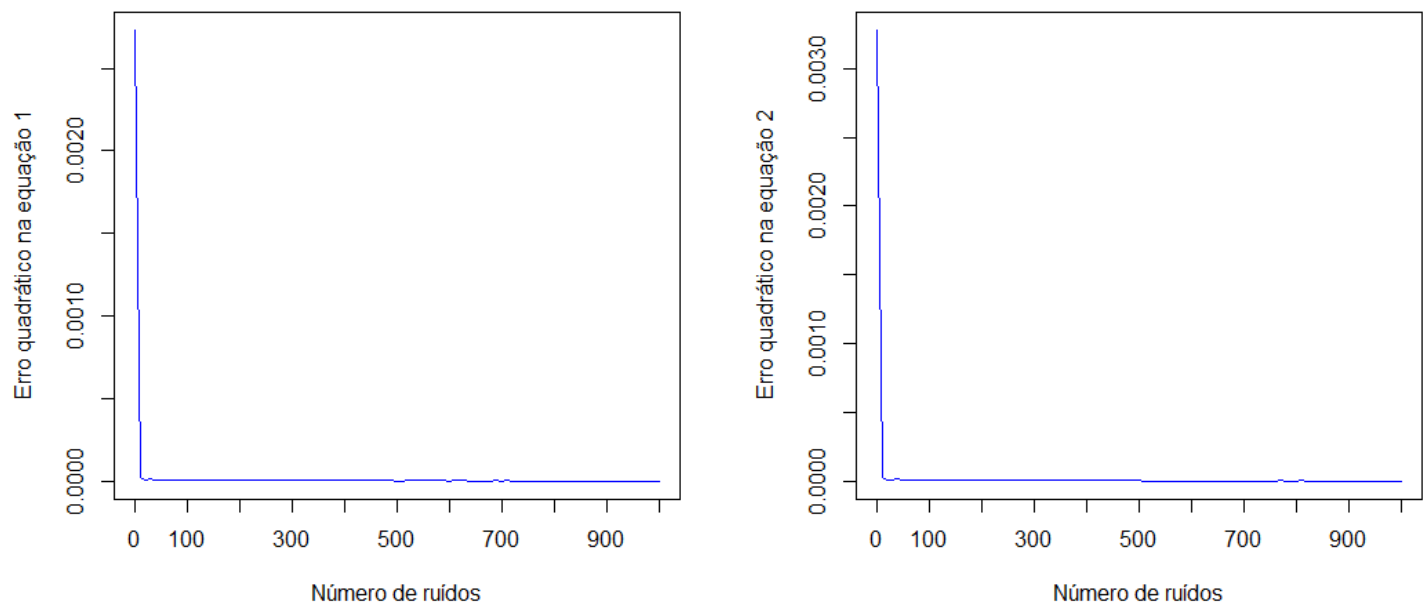

Figura 6.31: Erros quadráticos equações 1 e 2, ruído 1
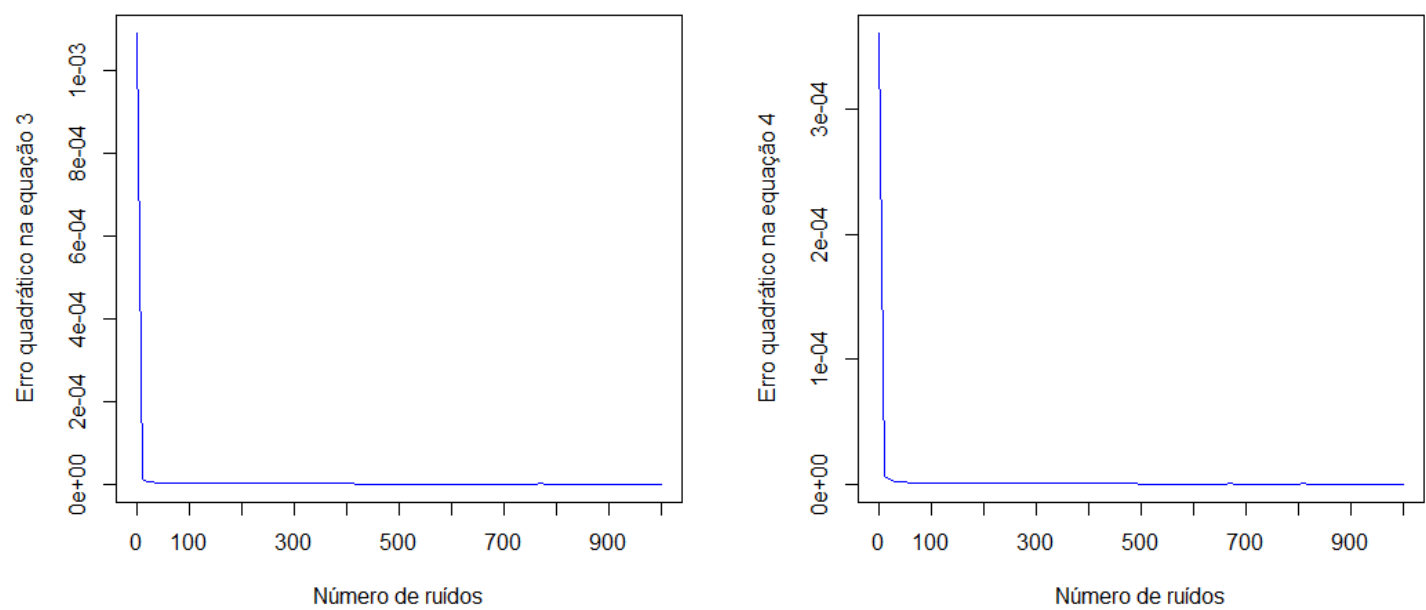

Figura 6.32: Erros quadráticos equações 3 e 4, ruído 1 

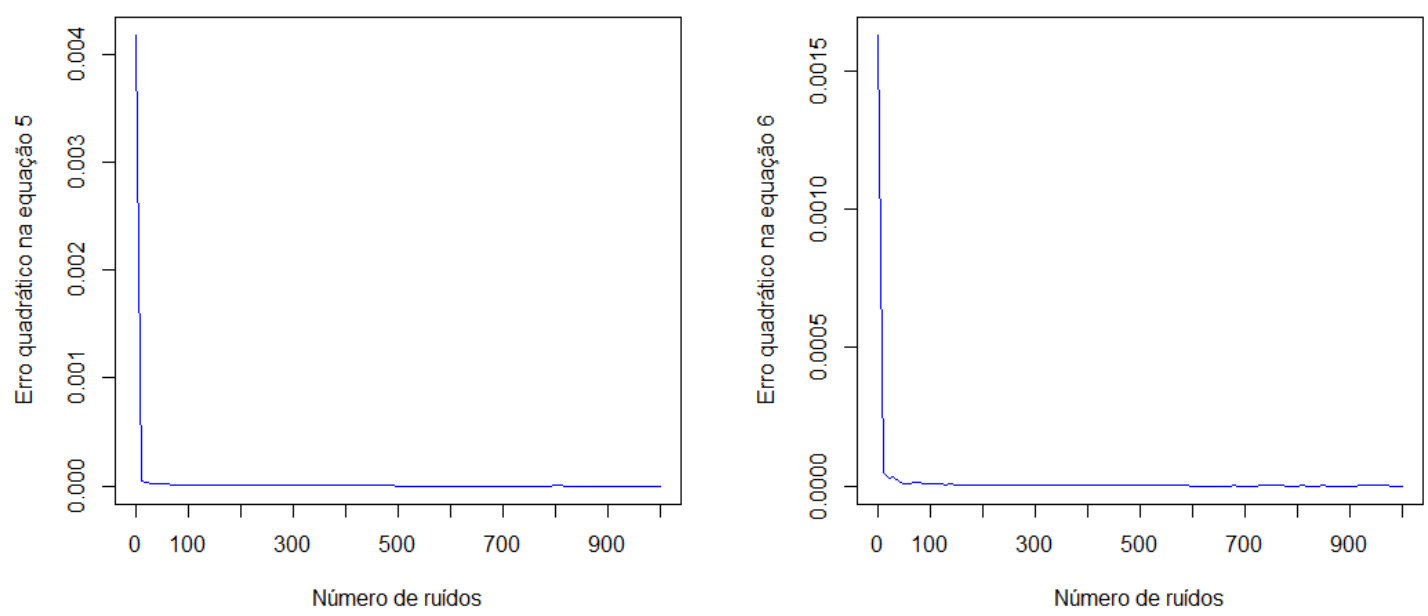

Figura 6.33: Erros quadráticos equações 5 e 6 , ruído 1
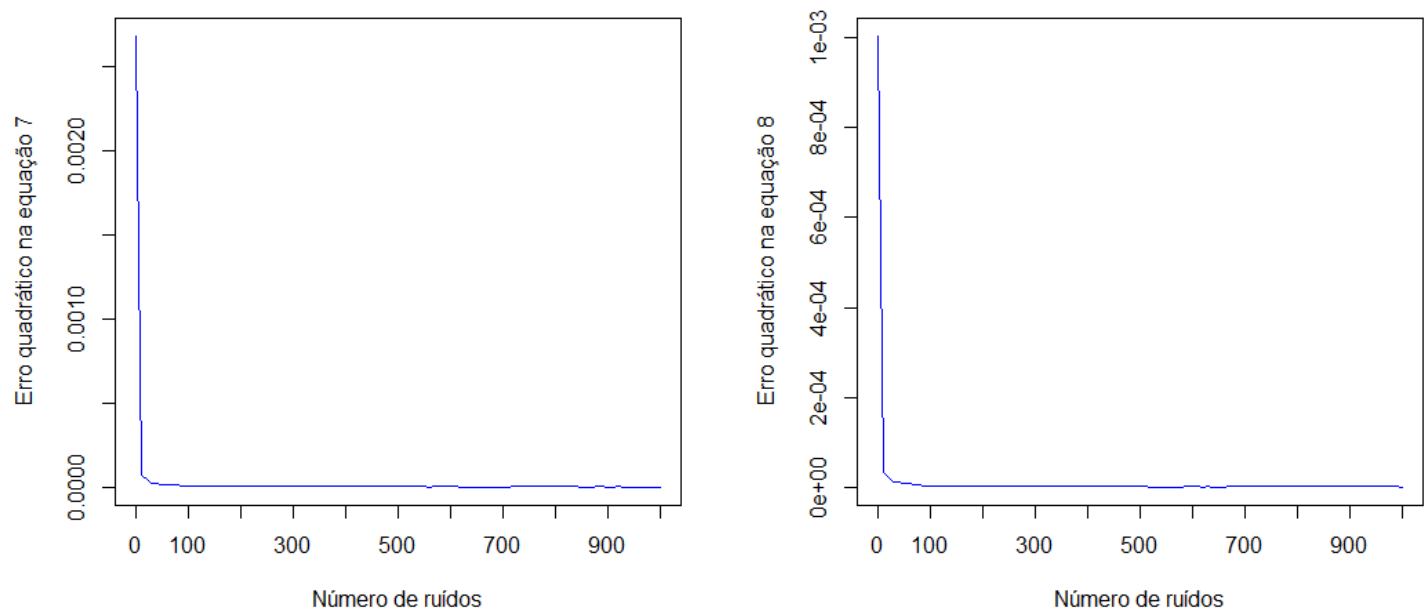

Figura 6.34: Erros quadráticos equações 7 e 8, ruído 1 

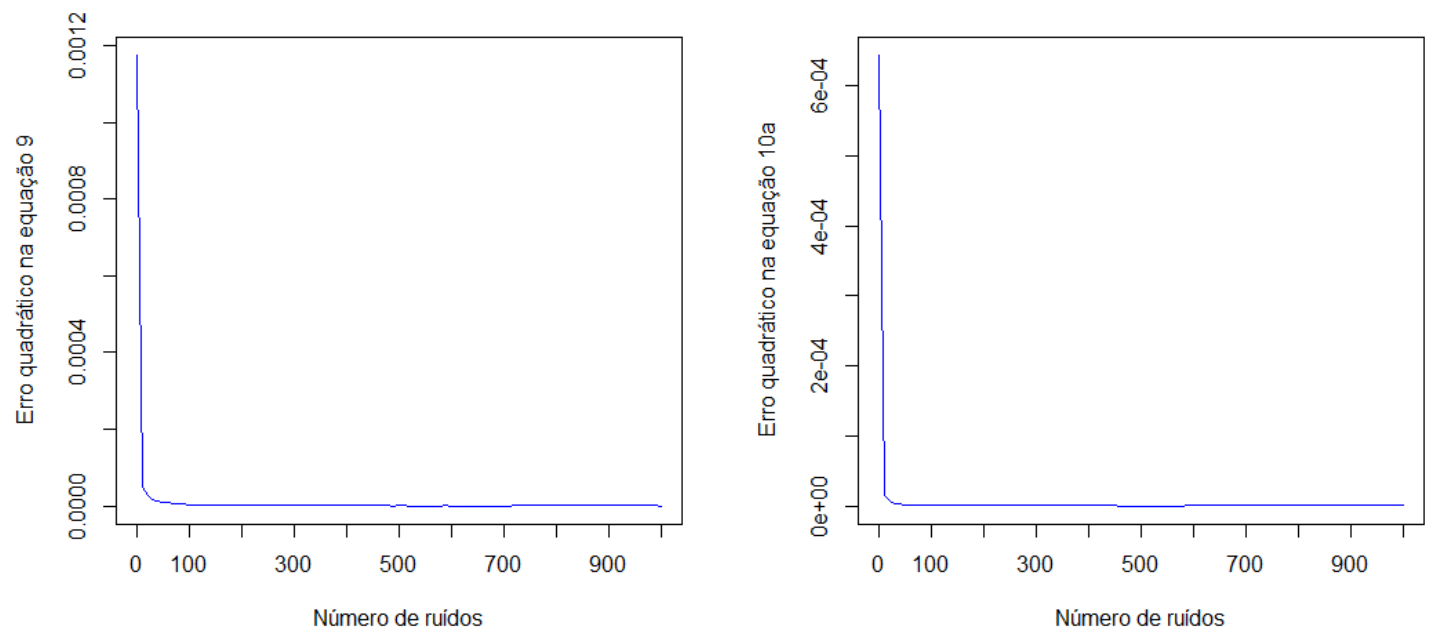

Figura 6.35: Erros quadráticos equações 9 e 10a, ruído 1
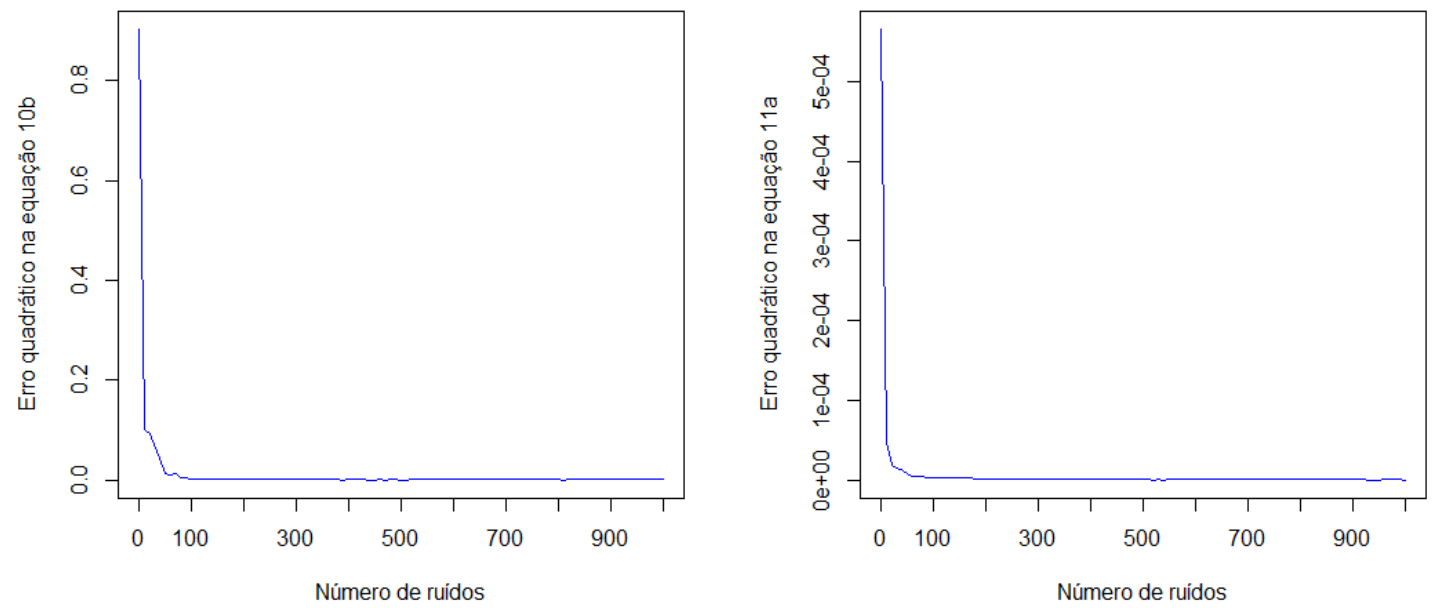

Figura 6.36: Erros quadráticos equações $10 b$ e 11a, ruído 1 

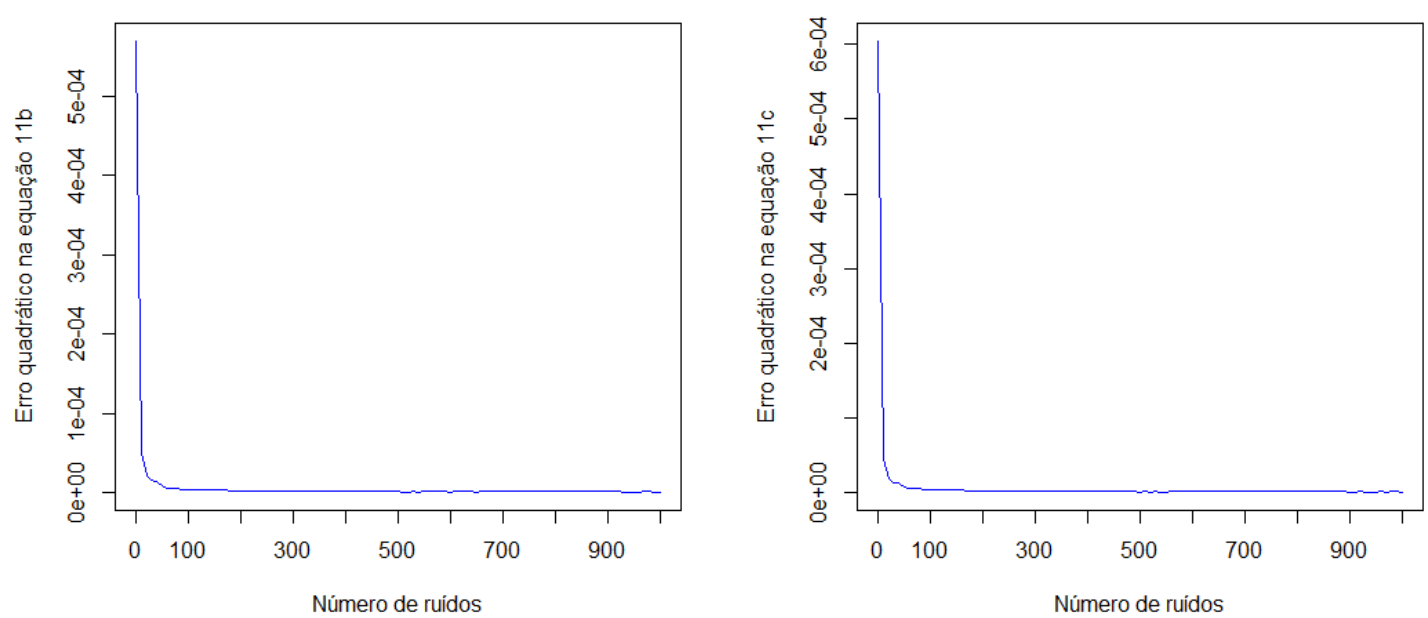

Figura 6.37: Erros quadráticos equações $11 b$ e 11c, ruído 1
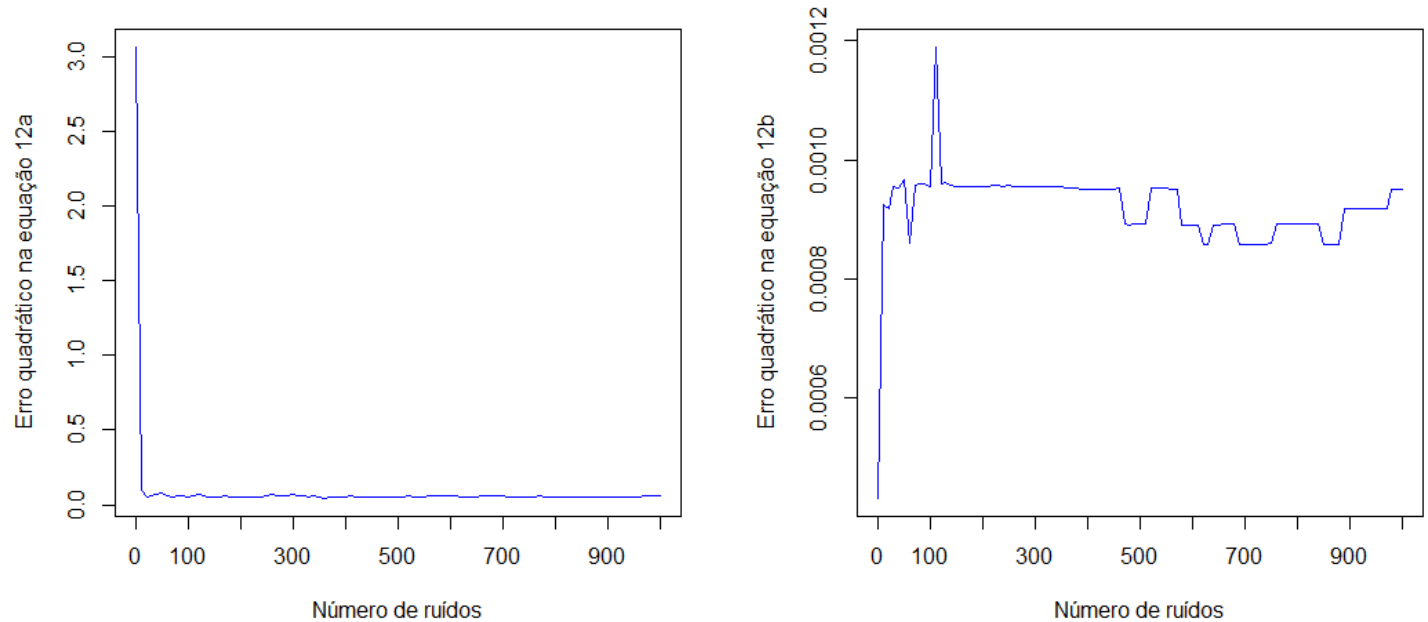

Figura 6.38: Erros quadráticos equações 12a e 12b, ruído 1 


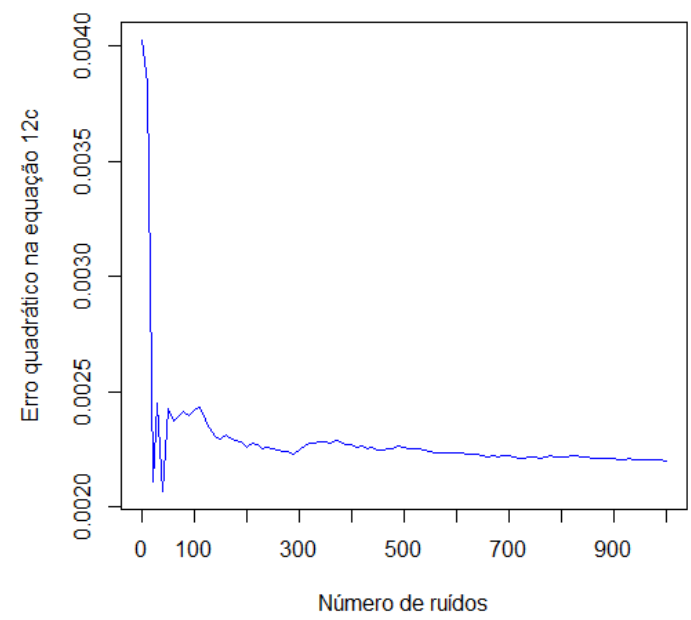

Figura 6.39: Erros quadráticos equações 12c, ruído 1
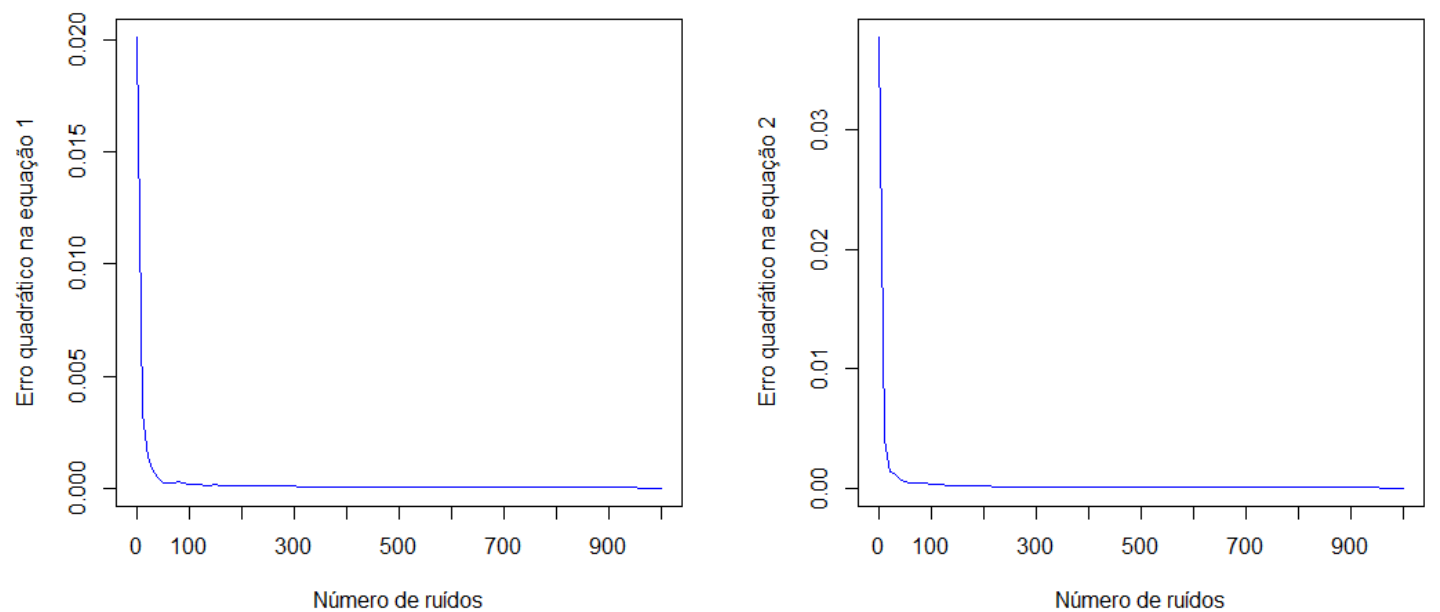

Figura 6.40: Erros quadráticos equações 1 e 2, ruído 2 

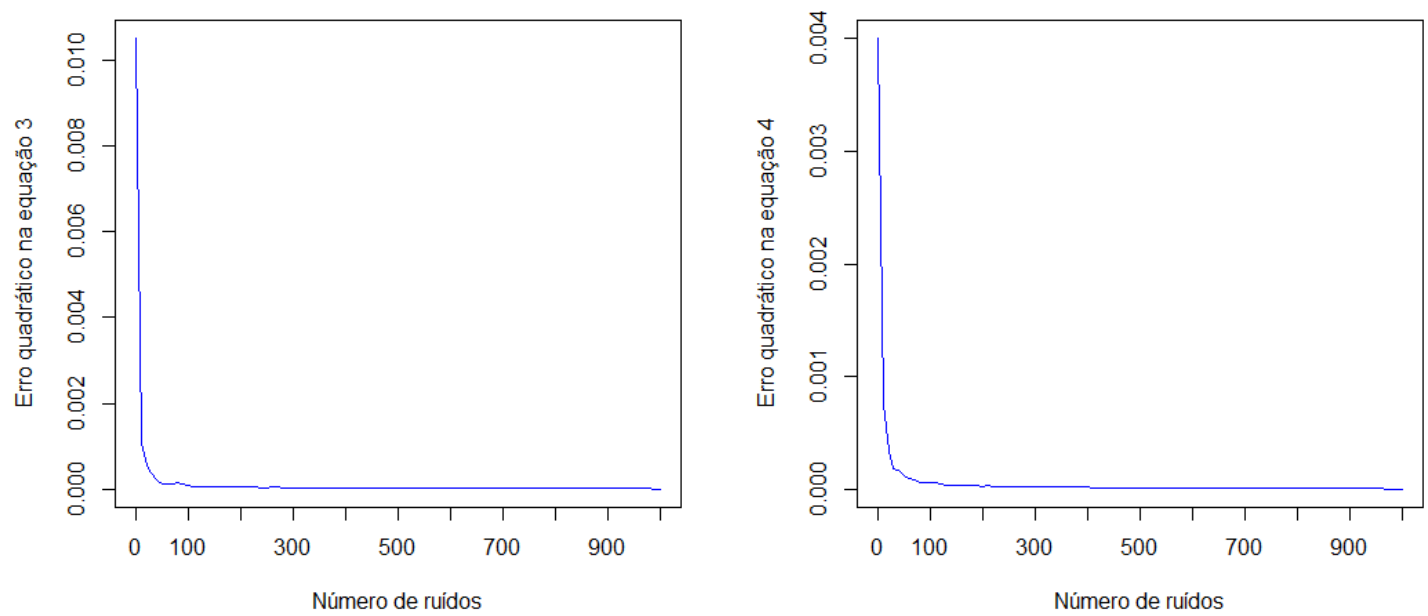

Figura 6.41: Erros quadráticos equações 3 e 4, ruído 2
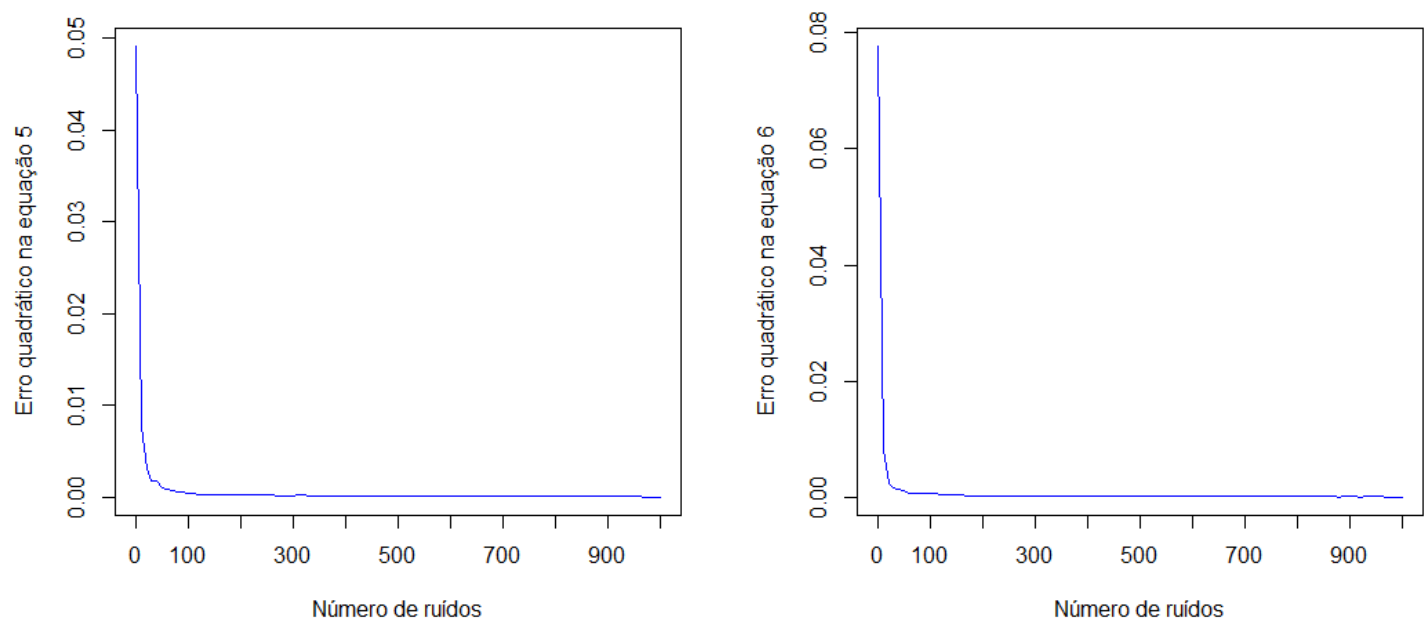

Figura 6.42: Erros quadráticos equações 5 e 6, ruído 2 

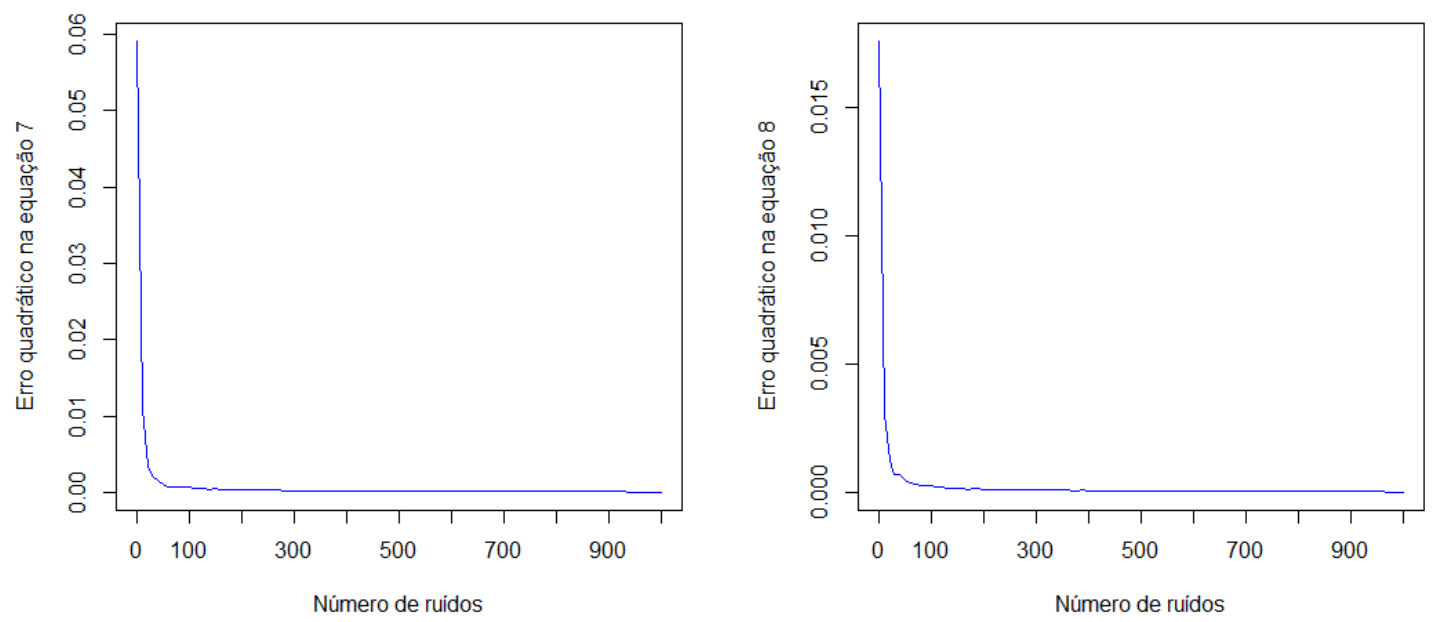

Figura 6.43: Erros quadráticos equações 7 e 8, ruído 2
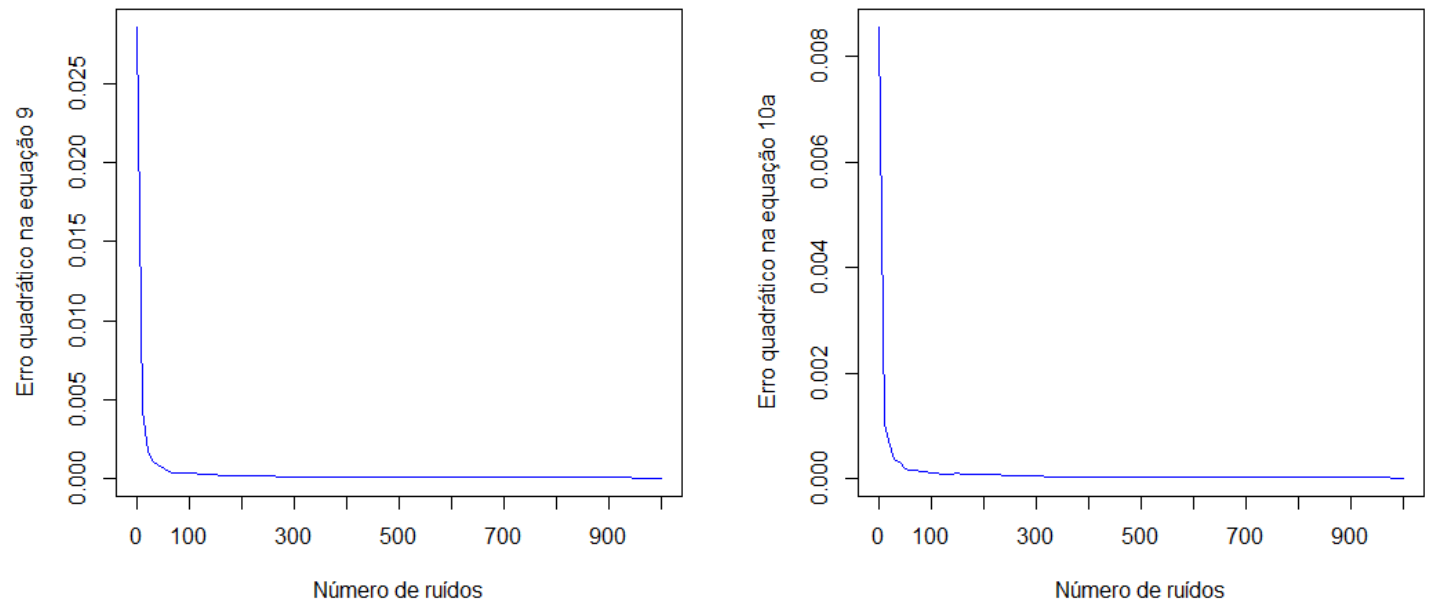

Figura 6.44: Erros quadráticos equações 9 e 10a, ruído 2 

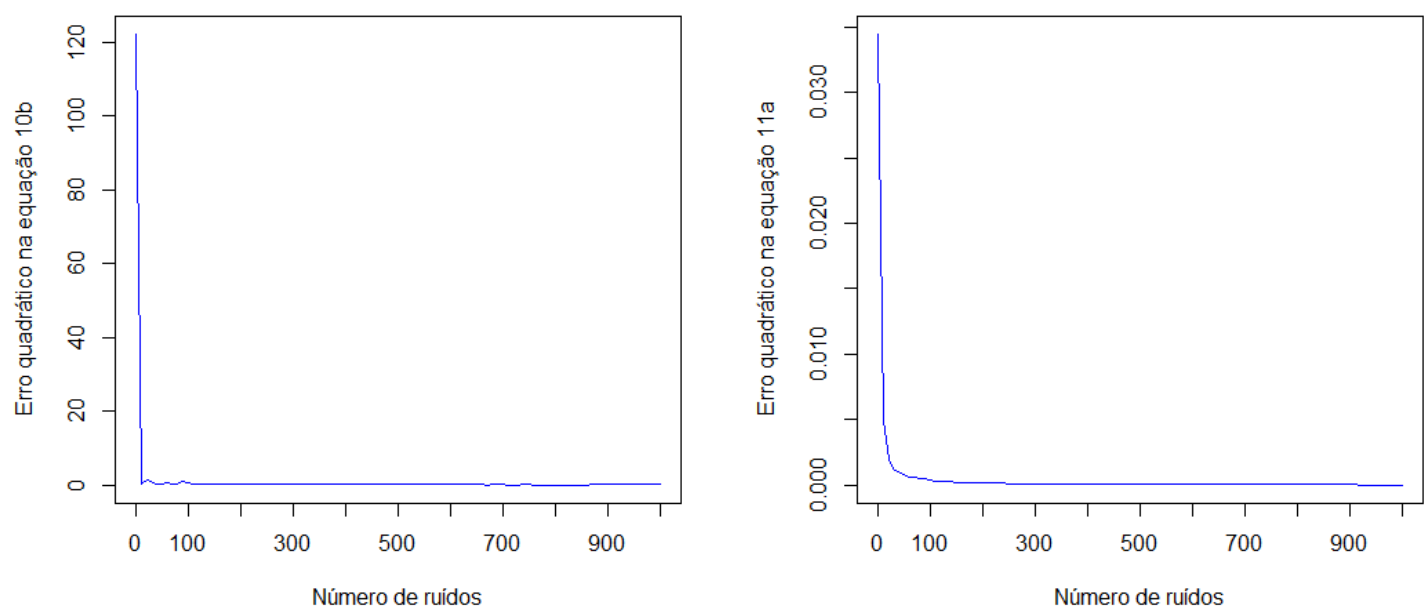

Figura 6.45: Erros quadráticos equações $10 b$ e 11a, ruído 2
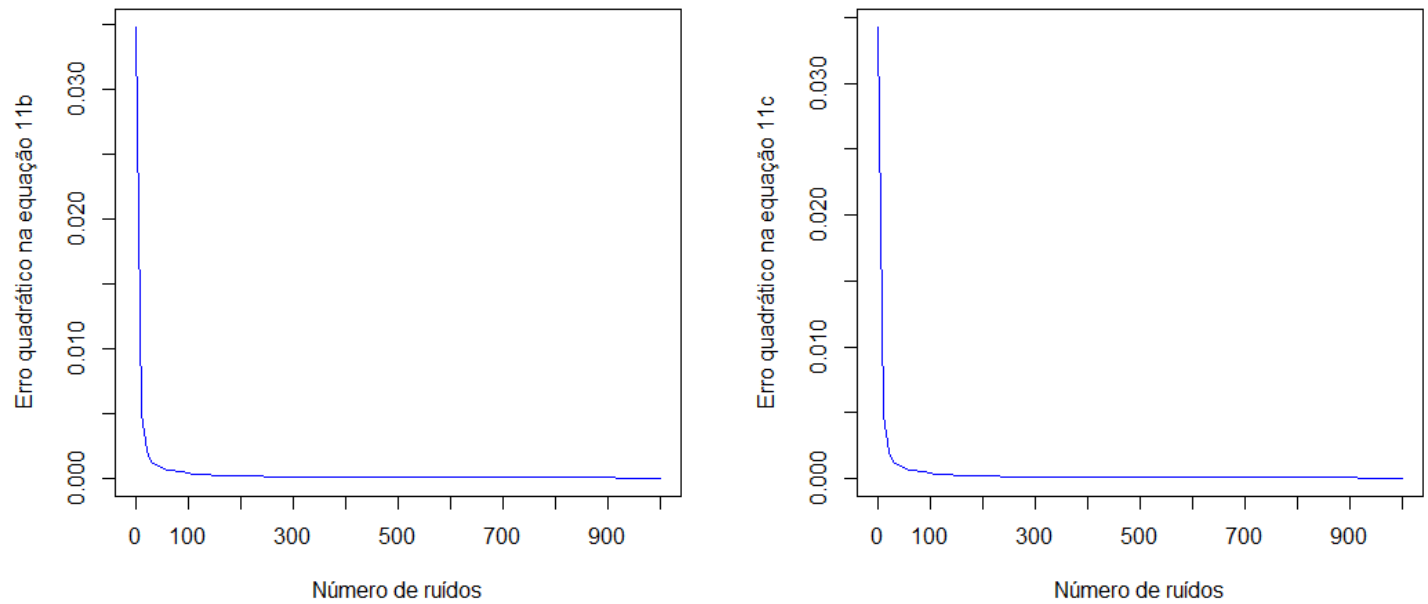

Figura 6.46: Erros quadráticos equações $11 b$ e 11c, ruído 2 

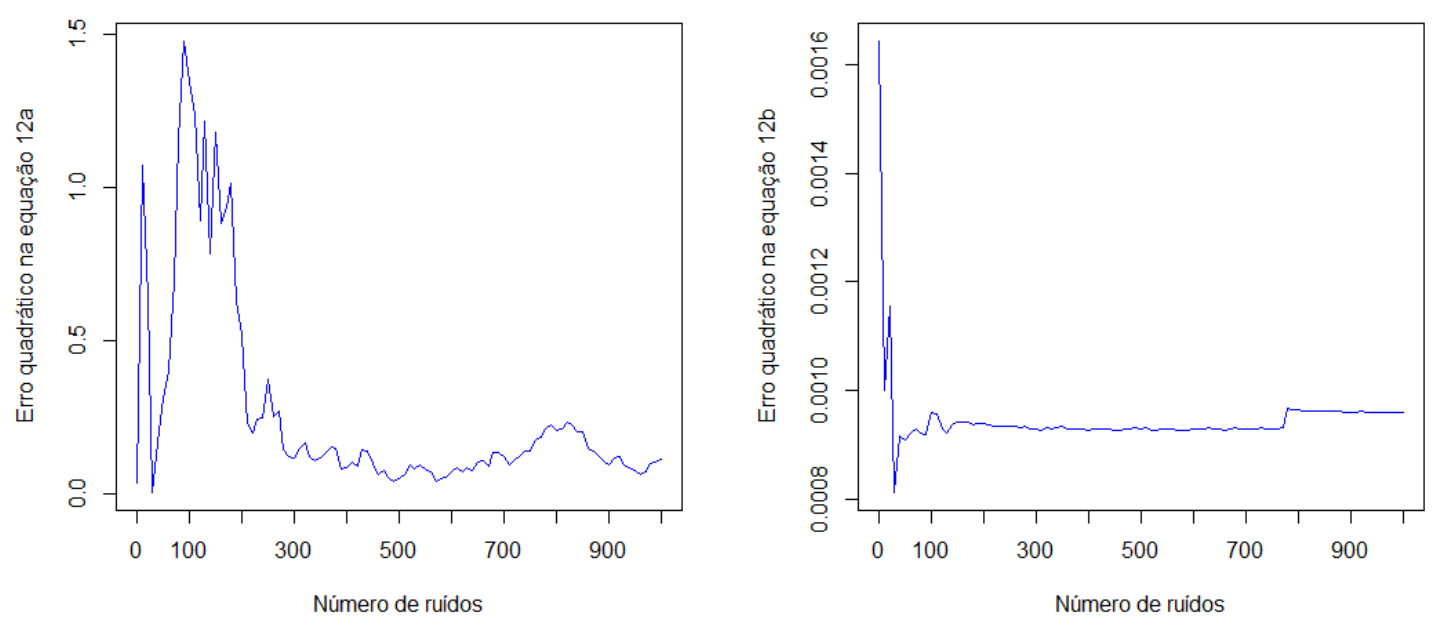

Figura 6.47: Erros quadráticos equações 12a e 12b, ruído 2

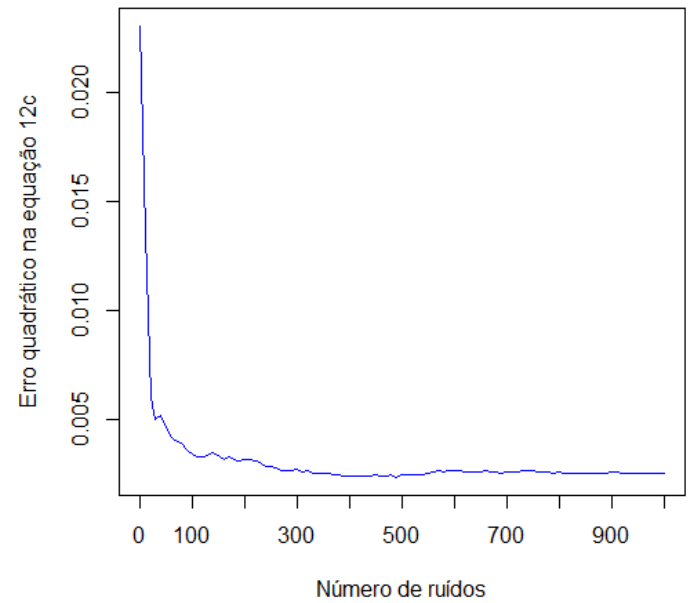

Figura 6.48: Erros quadráticos equações 12c, ruído 2 

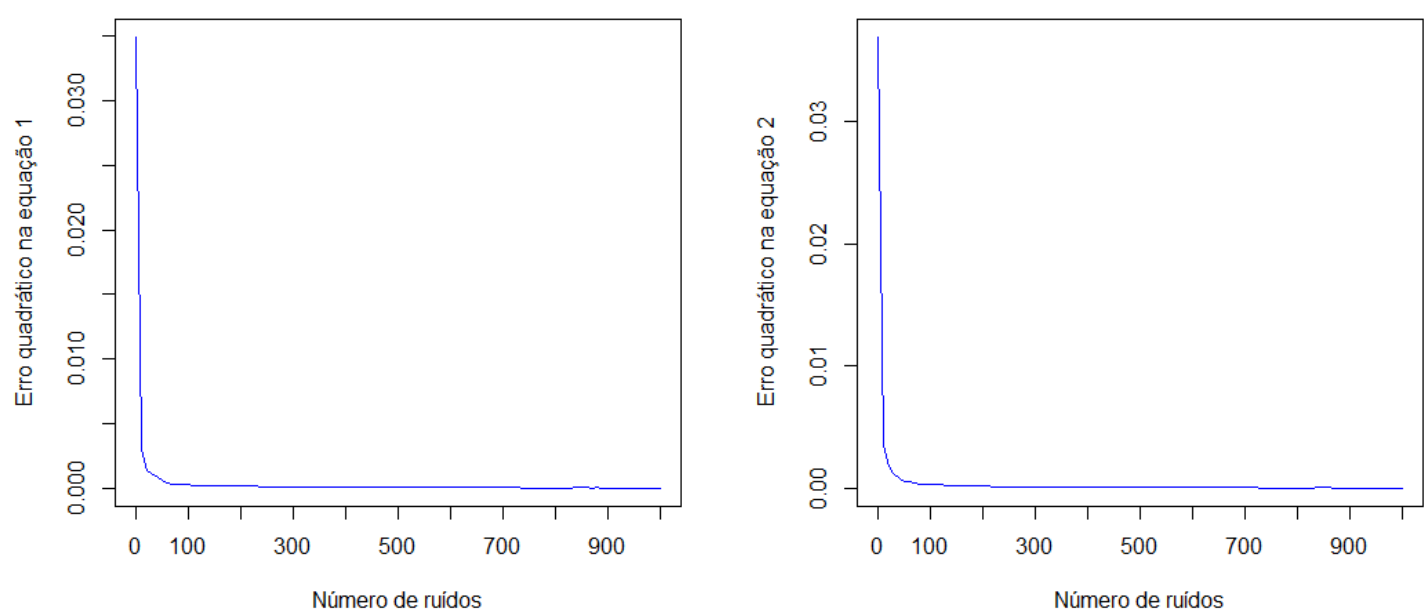

Figura 6.49: Erros quadráticos equações 1 e 2, ruído 3
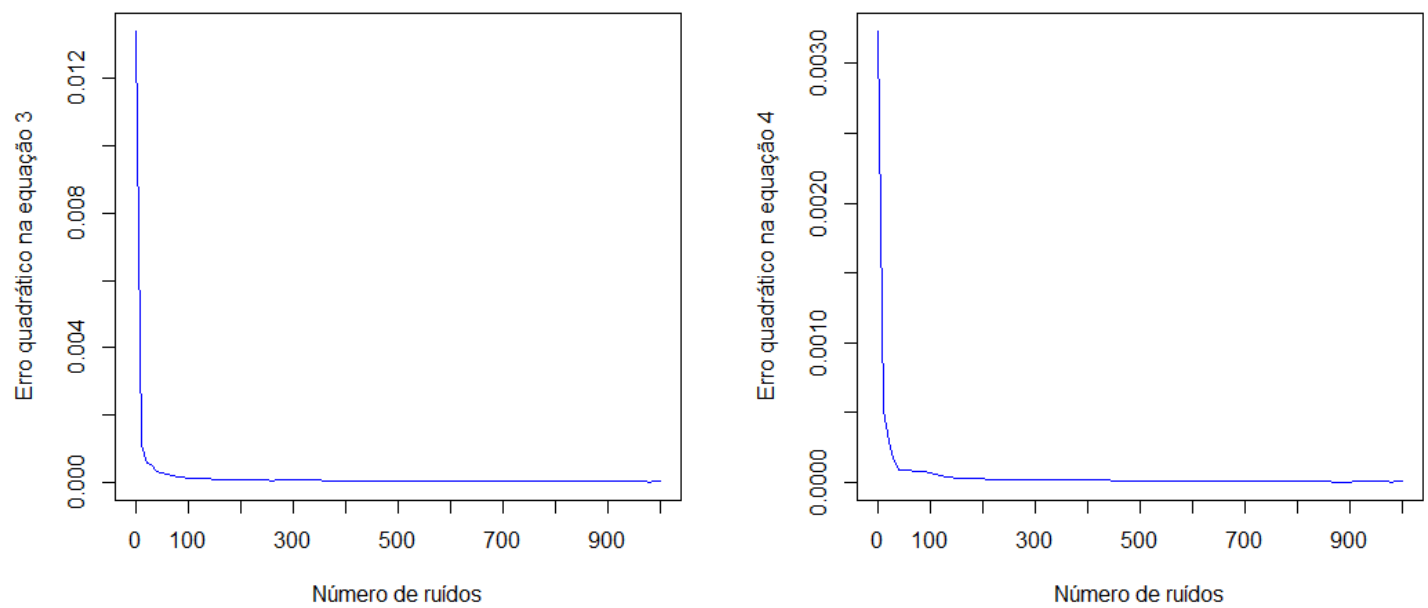

Figura 6.50: Erros quadráticos equações 3 e 4, ruído 3 

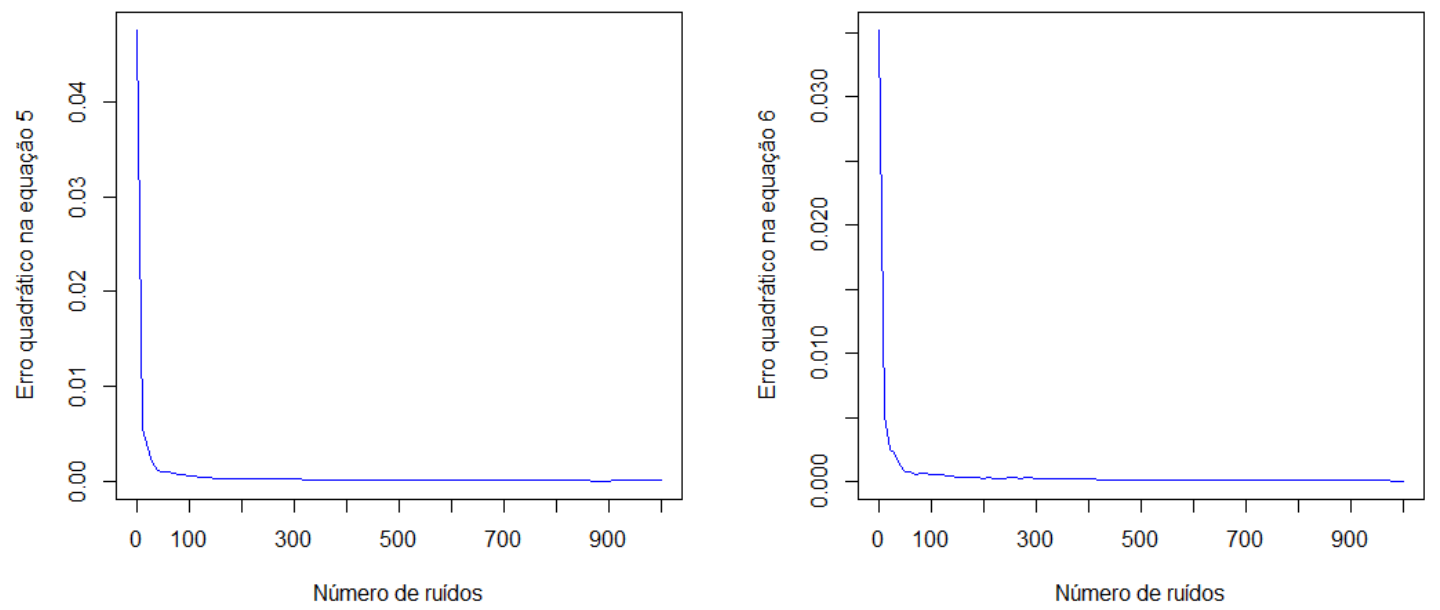

Figura 6.51: Erros quadráticos equações 5 e 6, ruído 3
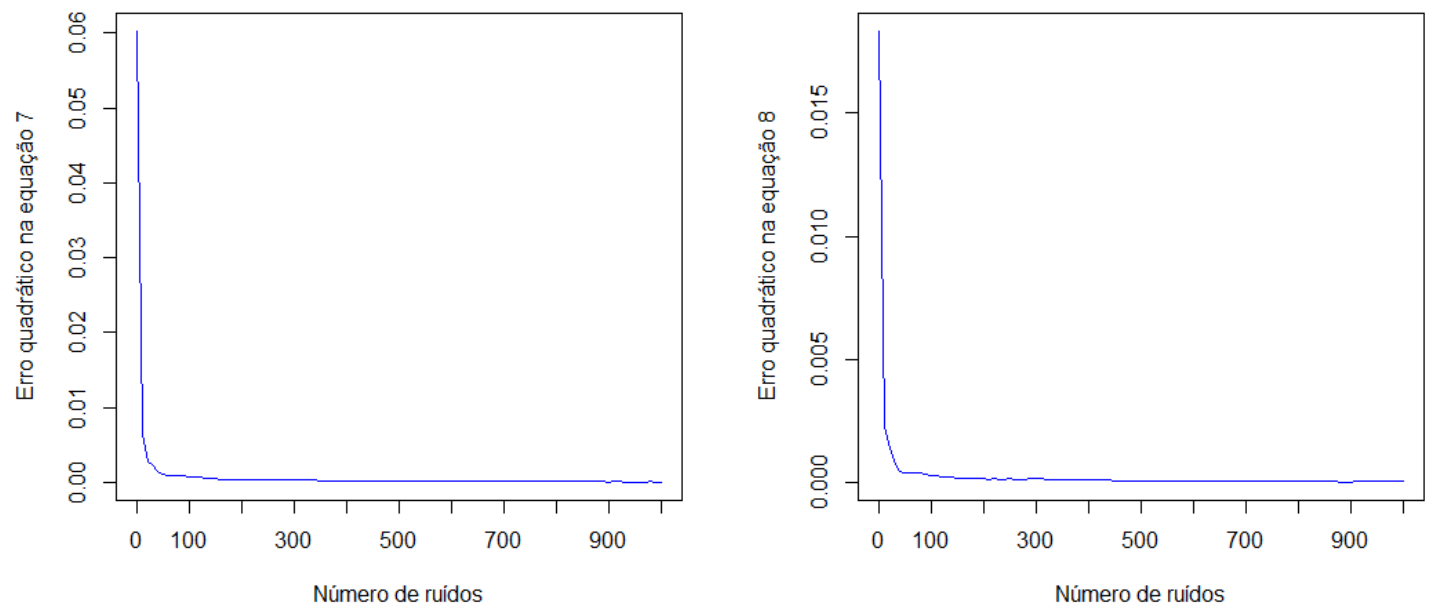

Figura 6.52: Erros quadráticos equações 7 e 8, ruído 3 

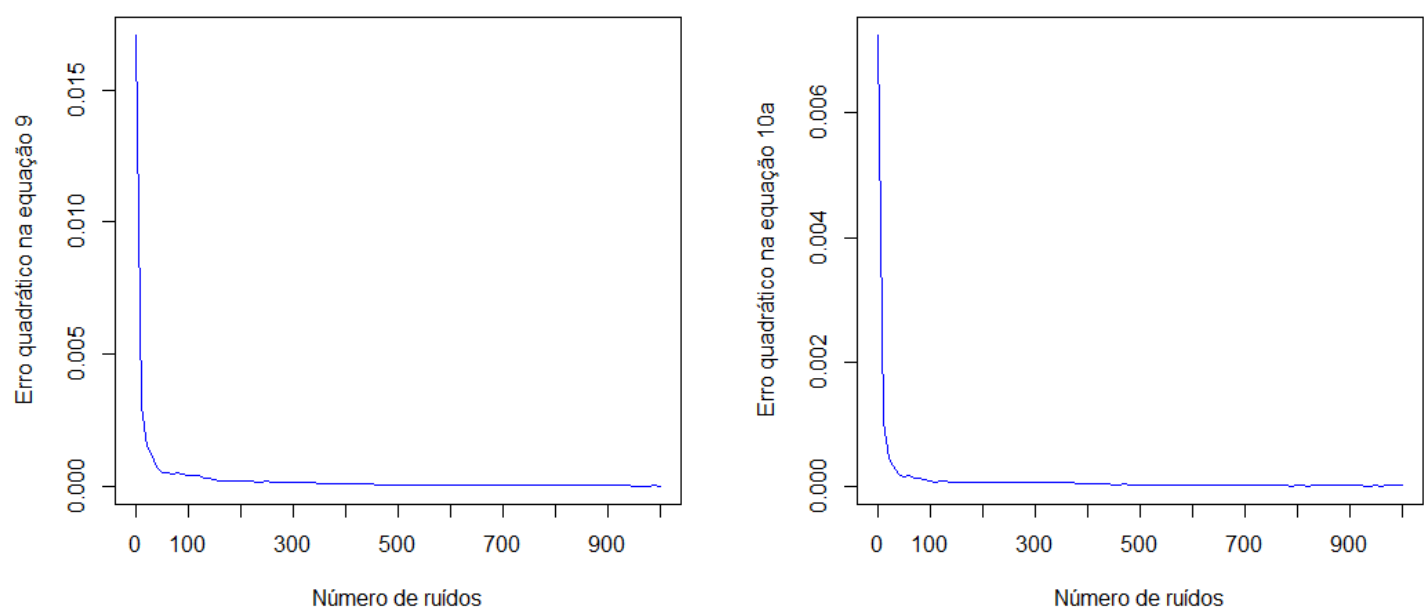

Figura 6.53: Erros quadráticos equações 9 e 10a, ruído 3
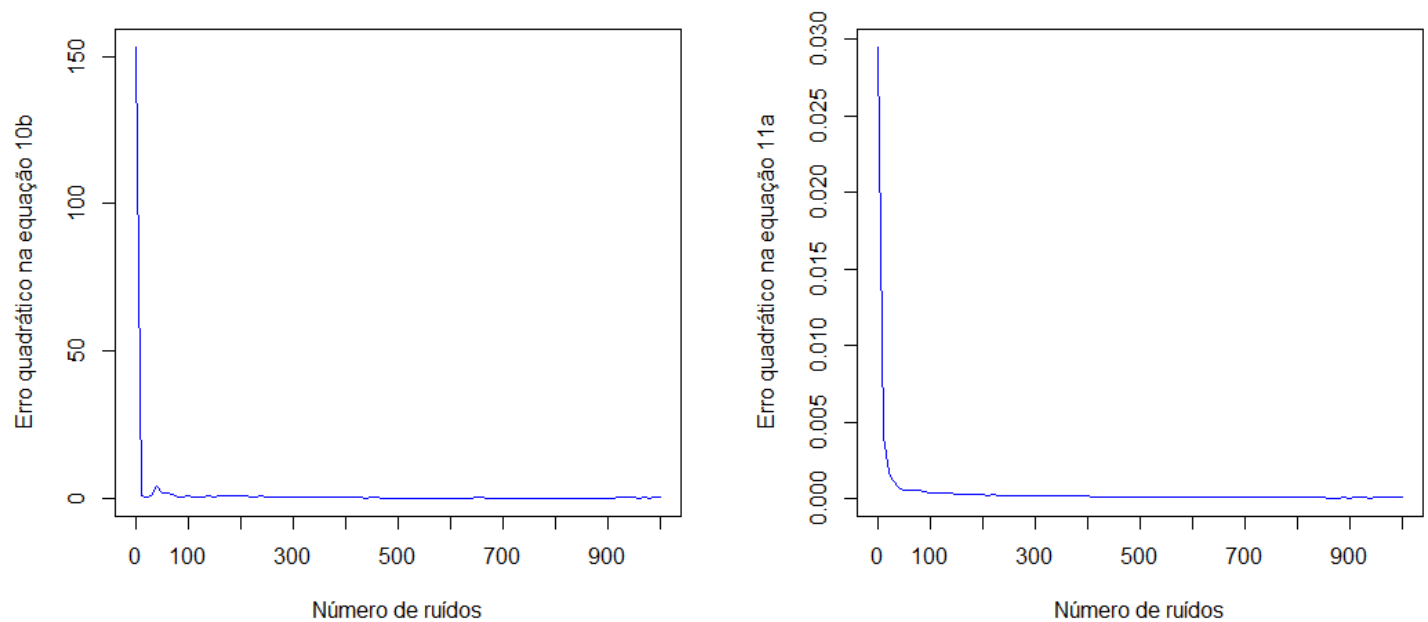

Figura 6.54: Erros quadráticos equações $10 b$ e 11a, ruído 3 

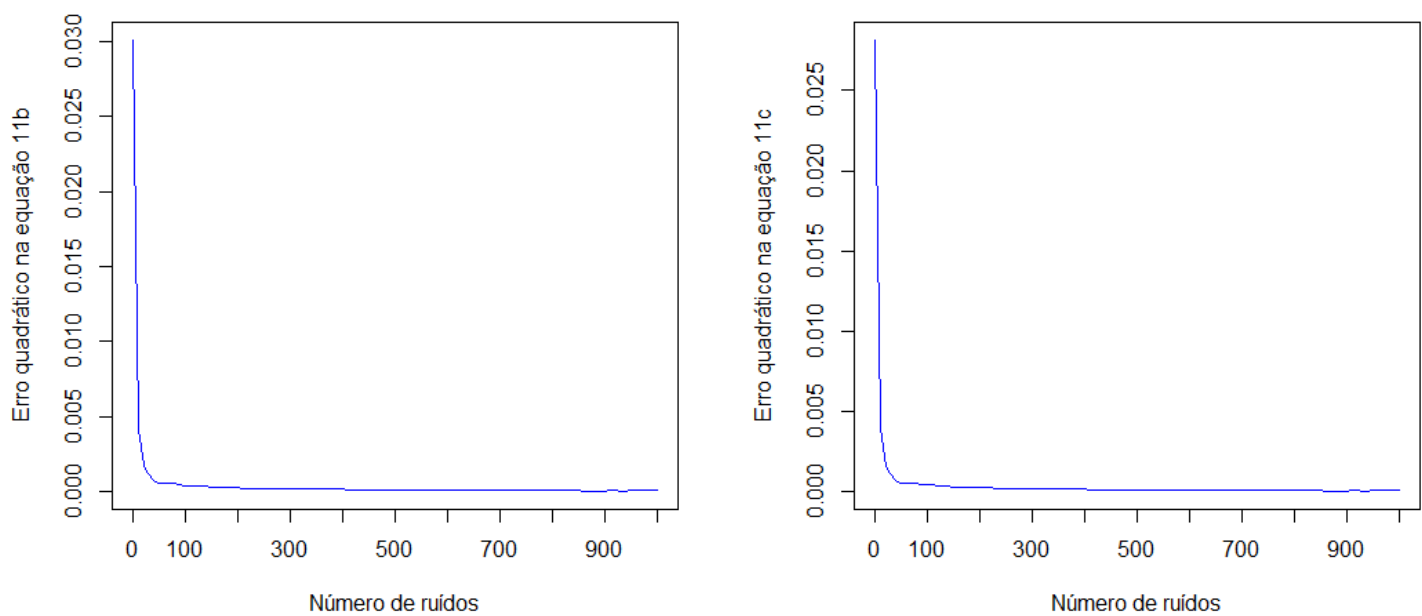

Figura 6.55: Erros quadráticos equações $11 b$ e 11c, ruído 3
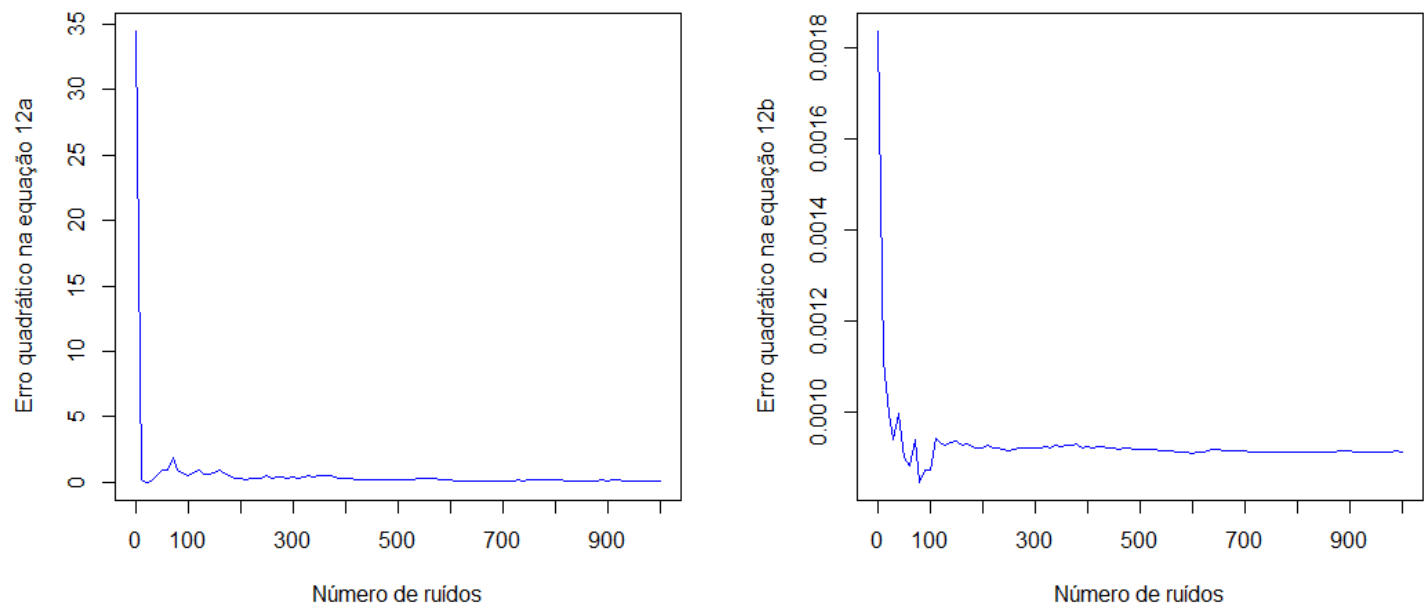

Figura 6.56: Erros quadráticos equações 12a e 12b, ruído 3 


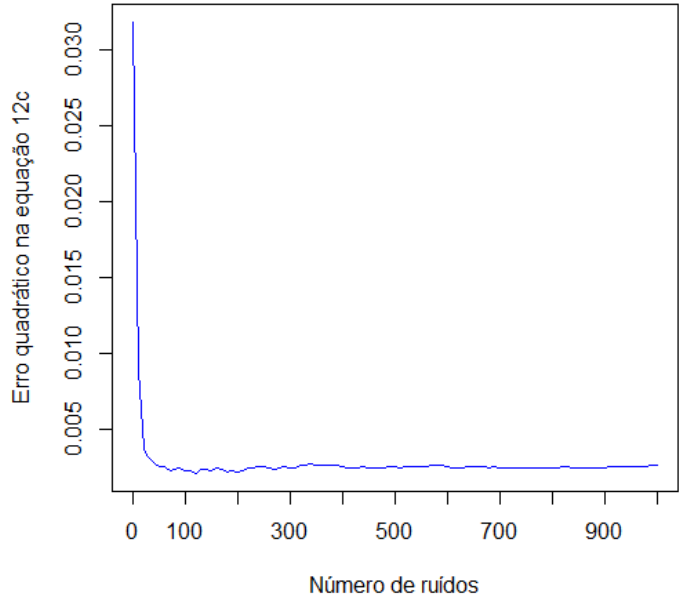

Figura 6.57: Erros quadráticos equações 12c, ruído 3 


\section{Capítulo 7}

\section{Considerações Finais}

Neste trabalho avaliamos o desempenho dos estimadores, com integrais, para os coeficientes funcionais da $\operatorname{EDP}$ (5.1). Vimos que na aplicação dos estimadores podem ocorrer divisão por zero quando o determinante $\operatorname{det}\left(\alpha, \beta, \gamma, \gamma^{\prime}\right)$ for igual a zero. Esse problema pode ser contornado pela escolha de um conjunto diferente de soluções, utilizadas na aplicação dos estimadores. Observamos que os estimadores com integrais utilizam toda a informação contida nas soluções, já os estimadores diretos utilizam a informação relativa a um valor fixo de $t$. Isso faz com que o desempenho dos estimadores diretos seja dependente da escolha de $t$. Na comparação dos dois grupos de estimadores vimos que para soluções sem ruídos os estimadores tem desempenho equivalentes. Já na presença de ruído nas soluções, o desempenho dos estimadores com integrais é superior ao dos diretos. Assim vemos que os estimadores com integrais apresentam características melhores que dos estimadores diretos.

\subsection{Sugestões para Pesquisas Futuras}

Uma melhoria possível no procedimento dos estimadores é aplicar um filtro para reduzir o ruído das soluções. Assim uma possível pesquisa seria a escolha dos possíveis filtros a serem aplicados.

Um problema encontrado na implementação dos estimadores é o cálculo das integrais (5.4), (5.5) e (5.6). Devido ao ruído, não é qualquer método numérico de integração que consegue calcular essas integrais. Então uma pesquisa dos métodos de integração mais adequados seria importante.

Por último temos o problema do cálculo das primeira e segunda derivadas da função $\gamma$. Neste trabalho passamos uma spline cúbica para obter uma aproximação dessas derivadas, e auxiliar no cálculo das integrais. Assim, outra sugestão de pesquisa seria métodos para derivação numérica e métodos para passar uma spline, por exemplo spline com suavização 


\section{Referências Bibliográficas}

[Blo14] Victor A Bloomfield. Using R for Numerical Analysis in Science and Engineering. CRC Press, 1 edição, 2014. 41, 42

[CM12] Karline Soetaert; Jeff Cash e Francesca Mazzia. Solving Differential Equations in R. Springer, 1 edição, 2012. 41

[dM11] Jose C S de Miranda. Estimation of functional coefficients in partial differential equations. Em Recent Advances and Related Topics in Functional Data Analysis, páginas 219-224. Physia-Verlag, 2011. 1, 2, 25

[Pol02] Andrei D Polyanin. Handbook of Linear Partial Differential Equations for Engineers and Scientists. CHAPMAN and HALL/CRC, 1 edição, 2002. 14, 31

[PR05] Y Pinchover e J Rubenstein. An Introduction to Partial Differential Equations. Cambridge University Press, 1 edição, 2005. 14, 15

[PS10] Karline Soetaert; Thomas Petzold e R Woodrow Setzer. Solving differential equations in r: Package desolve. Journal of Statistical Software, 33:1-25, 2010. 41

[Sch91] William E Schiesser. The Numerical Method of Lines: Integration of Partial Differential Equations. Academic Press, 1 edição, 1991. 15, 40

[SG09] William E Schiesser e Graham W Griffiths. A Compendium of Partial Differential Equation Models - Method of Lines Analysis with Matlab. Cambridge University Press, 1 edição, 2009. 15,40

[Str08] Walter A Strauss. Partial Differential Equations: An Introduction. Springer, 2 edição, 2008. 14

[Tho95] J W Thomas. Numerical Partial Differential Equations: Finite Difference Methods. Springer Verlag, 1 edição, 1995. 15

[WC10] Andrew T A Wood e Grace Chan. Simulation of stationary gaussian processes in $[0,1]^{2}$. Journal of Computational and Graphical Statistics, 3:409-432, 2010. 23 